\title{
Olfactory Perception and Physiology in Drosophila melanogaster
}

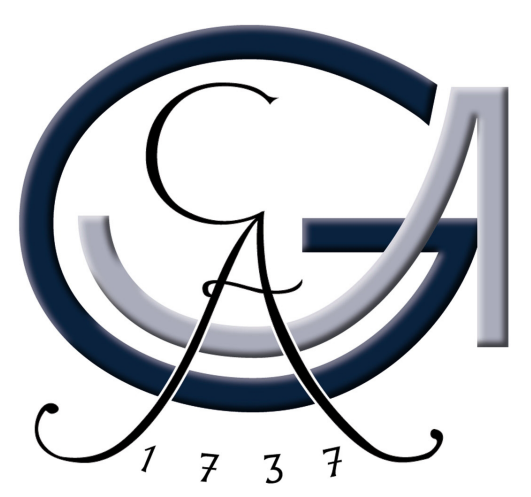

\author{
Dissertation \\ for the award of the degree \\ "Doctor rerum naturalium" \\ of the Georg-August-Universität Göttingen
}

\author{
submitted by \\ Jonas Barth \\ from \\ Büttelborn, Germany
}

Göttingen, 2013 
PhD Committee Member:

Prof. Dr. André Fiala (Supervisor, Reviewer)

Dept. Molecular Neurobiology of Behavior

Johann-Friedrich-Blumenbach Institute for Zoology and Anthropology, Georg-August-University Göttingen

PhD Committee Member:

Prof. Dr. Andreas Wodarz (Reviewer)

Stem Cell Biology, Dept. of Anatomy and Cell Biology

Göttingen Center of Molecular Biosciences

Georg-August-University Göttingen

PhD Committee Member:

Prof. Dr. Andre Fischer

Laboratory for Aging and Cognitive Diseases

European Neuroscience Institute Göttingen

Thesis submission:

March 31 ${ }^{s t}, 2013$

Date of the oral examination: May $16^{\text {th }}, 2013$ 


\section{Declaration of academic honesty}

I hereby declare that I have prepared this PhD thesis entitled "Olfactory Perception and Physiology in Drosophila melanogaster", on my own, using only the materials (devices) cited and with the help of the persons as indicated.

This work has not been submitted in equal or similar form to another examination office and has not been published.

Göttingen, March $31^{\text {st }}, 2013$ 


\section{Contents}

\begin{tabular}{ll}
\hline 1. Introduction & 8
\end{tabular}

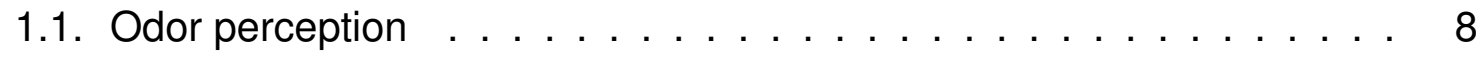

1.2. Generalization and Discrimination $\ldots \ldots \ldots \ldots$

1.2.1. Absolute versus differential conditioning . . . . . . . . 11

1.2.2. Sensory preconditioning . . . . . . . . . . . . 12

1.2.3. Discrimination of olfactory signals . . . . . . . . . . 13

1.3. Drosophila melanogaster as a model organism . . . . . . . . . 16

1.3.1. Binary expression systems . . . . . . . . . . . . 17

1.3.2. Manipulation of neuronal activity . . . . . . . . . 19

1.3.3. Monitoring of neuronal activity using in-vivo calcium imaging . 22

1.4. The olfactory system of the fly. . . . . . . . . . . . . . 26

1.4.1. Peripheral detection of odorants at the sensory organ . . . 26

1.4.2. Primary processing of olfactory information in the antennal lobe 30

1.4.3. Sparse coding of odors in the mushroom body . . . . . . . 33

1.5. Olfactory learning in Drosophila melanogaster $\ldots \ldots \ldots$

1.5.1. Neuronal plasticity in the antennal lobe . . . . . . . . 42

1.5.2. Learning induced plasticity in the mushroom body . . . . . . 4 43

1.6. Aim of the study $\ldots \ldots \ldots \ldots \ldots \ldots \ldots$

2. Materials and Methods $\quad \mathbf{5 0}$

2.1. Materials . . . . . . . . . . . . . . . . 50

2.1.1. Fruit flies and bacteria . . . . . . . . . . . . . . 50

2.1.2. Constructs and plasmids . . . . . . . . . . . . 51

2.1.3. Media . . . . . . . . . . . . . . . . . . . 52

2.1.4. Reagents and consumables . . . . . . . . . . . 53

2.1.5. Odorants . . . . . . . . . . . . . . . . . 54 
2.1.6. Buffers and solutions. . . . . . . . . . . . . . . . . . 54

2.1.7. Enzymes . . . . . . . . . . . . . . . 56

2.1.8. Antibodies. . . . . . . . . . . . . . . . . . 57

2.1.9. Laboratory equipment . . . . . . . . . . . . . . . 57

2.2. Methods . . . . . . . . . . . . . . . . . . . . . . . . 59

2.2.1. Fly husbandry . . . . . . . . . . . . . . . . 59

2.2.2. Generation of transgenic flies . . . . . . . . . . . . 59

2.2.3. Immunohistochemistry . . . . . . . . . . . . . 63

2.2.4. Behavioral paradigms . . . . . . . . . . . . 65

2.2.5. In-vivo calcium imaging . . . . . . . . . . . . . . 69

3. Results 75

3.1. Establishing the olfactory learning paradigm in the laboratory . . . 75

3.2. Sensory preconditioning experiments $\ldots \ldots \ldots \ldots 78$

3.3. Determining odorant concentrations . . . . . . . . . 80

3.4. Generalization learning of similar odorants . . . . . . . . . . . 84

3.5. $\mathrm{Ca}^{2+}$-imaging of the three used odorants in the antennal lobe . . . . 86

3.6. Discrimination learning of similar odorants . . . . . . . . . . . 90

3.7. Blocking of synaptic transmission in inhibitory local interneurons of the antennal lobe . . . . . . . . . . . . . . . . . . 91

3.7.1. Anatomy of two populations of local interneurons labelled by NP1227- and NP2426-GAL4 . . . . . . . . . . . . . . 92

3.7.2. General behavioral performance of flies with blocked synaptic transmission in two populations of local interneurons . . . . . . 95

3.7.3. Absolute learning . . . . . . . . . . . . . . . . . 102

3.7.4. Generalization learning of similar odorants . . . . . . . 103

3.7.5. Discrimination learning of non-similar odorants . . . . . . 105

3.7.6. Discrimination learning of similar odorants. . . . . . . . . 106

3.8. Different conditioning paradigms alter the olfactory preference. . . . 107

3.9. Differential training reduces odor generalization . . . . . . . . . 110

3.10. Discrimination of similar odorants depends on the choice during the test . . . . . . . . . . . . . . . . . . . . 111 


\section{Contents}

3.11. Changes in neuronal activity in the mushroom body as a result to learning: first steps . . . . . . . . . . . . . . . . . . . . . 113

3.12. New tools for future studies: generation of new fly strains for optical imaging . . . . . . . . . . . . . . . 1117 3.12.1. Expression of red fluorescing calcium indicators . . . . . . . 119 3.12.2. A more versatile application of the GCaMP3.0 sensor . . . . . 122

4. Discussion

4.1. Sensory preconditioning - an attempt to investigate changes in olfactory acuity . . . . . . . . . . . . . . . . . . . . . . . . . . . . . . 124

4.2. The chemical similarity of 1-Oct and 3-Oct results in a behaviorally and physiologically determined similarity . . . . . . . . . . . . . . . . . 126

4.3. Learning to discriminate similar odorants. . . . . . . . . . . . . . . . . 128

4.4. Olfactory discrimination in the antennal lobe . . . . . . . . . . . . . . 129

4.5. Increased olfactory "acuity" as a result of differential training . . . . . . 132

4.6. Asymmetric differential conditioning of 1-Oct and 3-Oct . . . . . . . . 133

4.7. A possible localization of learned discrimination - A first attempt . . . . 135

$\begin{array}{ll}\text { 5. Summary } & 142\end{array}$

\begin{tabular}{ll}
\hline A. Abbreviations & 177
\end{tabular}

\begin{tabular}{lr}
\hline B. Fly crosses & 180
\end{tabular}

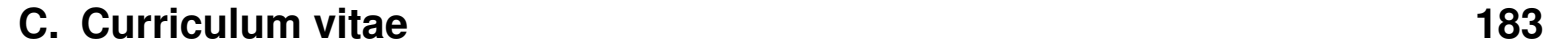




\section{List of Figures}

1.1. The GAL4/UAS system … . . . . . . . . . . . . . 18

1.2. Mechanism of shibirets function . . . . . . . . . . . . . . . 21

1.3. Schematics of different GECls $\ldots \ldots \ldots . . \ldots 24$

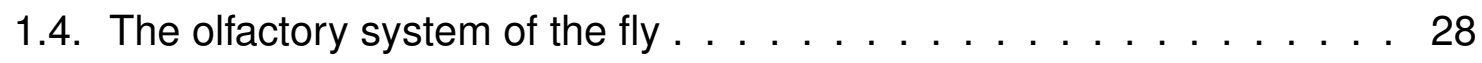

1.5. 3D-model of the mushroom body lobes . . . . . . . . . . 34

1.6. Schematic of the olfactory learning paradigm . . . . . . . . . 39

1.7. Different forms of memory . . . . . . . . . . . . . . 41

1.8. Scheme of the intrinsic and extrinsic mushroom body neurons during the different phases of learning . . . . . . . . . . . . 46

1.9. Chemical structures of 1 -Oct, 3 -Oct and $\mathrm{MCH} \ldots \ldots \ldots$

2.1. Photography of modified Tully-Quinn-Barrel . . . . . . . . 66

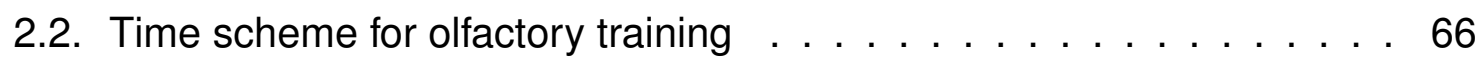

2.3. Scheme of fly preparation for imaging . . . . . . . . . 69

2.4. Scheme of the experimental design for mushroom body imaging . . 71

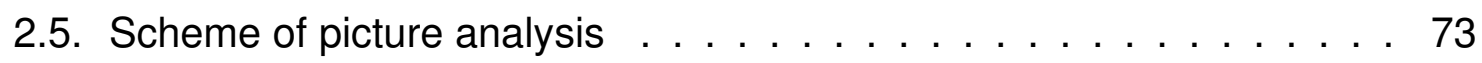

3.1. Calibration of the Tully-machine . . . . . . . . . . 76

3.2. Olfactory conditioning resulted in stable learning indices . . . . . 77

3.3. Test for the flies' ability for olfactory sensory preconditioning . . . . . 79

3.4. Test for the learnability of different odorant dilutions . . . . . . . . 81

3.5. Naïve responses towards odorants in changing dilutions . . . . . . 82

3.6. Final odor dilution determined by pre-experiments . . . . . . . 83

3.7. Generalization of similar odorants . . . . . . . . . . . . 85

3.8. $\mathrm{Ca}^{2+}$-dynamics measured in the antennal lobe - focal plane 1 . . . 86

3.9. $\mathrm{Ca}^{2+}$-dynamics measured in the antennal lobe - focal plane $2 \ldots$ 
3.10. Discrimination learning of similar odorants . . . . . . . . . . . . . . . . 90

3.11. Anatomy of LN1 interneurons . . . . . . . . . . . . . . . . . . . . . 93

3.12. Anatomy of LN2 interneurons . . . . . . . . . . . . . . . . . . . . . . . 95

3.13. Locomotor performance of flies with blocked synaptic transmission in NP1227-GAL4 and NP2426-GAL4 . . . . . . . . . . . . . . . . . . . 97

3.14. Shock avoidance of flies with silenced NP1227 and NP2426 neurons . 98

3.15. Odor preference of NP1227>Shits and NP2426>Shits . . . . . . . . . . 100

3.16. Absolute conditioning of NP1227>Shits . . . . . . . . . . . . . . . . 102

3.17. Generalization of 1-Oct and 3-Oct in NP1227>Shits flies . . . . . . . . 104

3.18. Discrimination of 1-Oct/3-Oct and $\mathrm{MCH}$ in NP1227>Shits flies . . . . . 105

3.19. Differential training of similar odorants in NP1227>Shits flies . . . . . . 106

3.20. Differential training increases odor discrimination . . . . . . . . . . . . 108

3.21. Timing of CS- presentation has no influence on the odorant preference after learning . . . . . . . . . . . . . . . . . . . . . . 109

3.22. Differential training reduces odor generalization . . . . . . . . . . . . . 110

3.23. Discrimination of 3-Oct and 1-Oct depends on the presented odorants in the test situation . . . . . . . . . . . . . . . . . . . 112

3.24. Neuronal activity pattern in the mushroom body . . . . . . . . . . . . . . . 114

3.25. Learning evoked changes in odorant representation in the mushroom body . . . . . . . . . . . . . . . . . . . . . 116

3.26. Vectormaps of UAS:R-GEC01.0 and mb247:R-GECO1.0 . . . . . . . 118

3.27. Evaluation of the expression levels of the UAS:R-GECO1.0 fly lines. . 119

3.28. Evaluation of mb247:R-GECO1.0 . . . . . . . . . . . . . . . . 121

3.29. Vectormap of LexOp:GCaMP3.0 . . . . . . . . . . . . . . . . . . . . . 122

3.30. Expression of LexOp:GCaMP3.0 in Or83b-lexA:VP16 flies . . . . . . . 123

4.1. Connectivity model of similar odorants in the calyx . . . . . . . . . . . 136

4.2. Hypothetical working model of cellular changes in response to absolute learning . . . . . . . . . . . . . . . . . . . . . . . . . 138

4.3. Hypothetical working model of cellular changes in response to differential learning . . . . . . . . . . . . . . . 141 


\section{Introduction}

\subsection{Odor perception}

The perception of chemical structures in the environment is a very important task for most animals throughout the whole animal kingdom. Almost every species known has a form of chemosensation in order to react to changes in the environment that might cause a thread or be beneficial for the organism. Chemical structures in the air can contain information of nearby food sources, predators or mating partners. Organisms do not only have to perceive the chemicals, but also to evaluate them and to find an appropriate behavioral response [Wilson \& Stevenson, 2006].

Chemotaxis of single-cellular organisms is the simplest example of such a behavior: bacteria react to concentration gradients of harmful or beneficial substances with an approach or avoidance behavior. These behavioral responses are mediated by chemosensation and intracellular signaling cascades [Wadhams \& Armitage, 2004]. These simple forms of responses to chemical stimuli, however, are only induced by a limited number of molecules and their respective concentrations or concentration changes dependent on the number of diverse receptors available. For higher organisms, the variety of chemicals that have to be perceived and evaluated is much higher. The environment contains a huge amount of mixtures of chemical structures that have to be perceptually combined in order to be evaluated. If the chemosensory system would be only responding to individual chemicals, the "olfactory space" of the animal would be very limited and an appropriate response to the variety of mixtures impossible.

The combination of all stimuli in the environment has to be perceived before an evaluation of the situation can be made. Additionally, the environment includes many sources of chemical compounds that continuously surround an animal. This background has to be distinguished from a relevant, instantaneous occurring odor source. 


\section{Introduction}

In vertebrates and invertebrates, the perception and evaluation of chemosensory stimuli is therefore located in different parts of the nervous systems [Wilson \& Stevenson, 2006]. Interestingly, the neuronal circuitry mediating the peripheral detection of volatile chemicals by the olfactory system is strongly conserved throughout evolution [Ache \& Young, 2005]. On the first level of sensory input, the different chemical components of the surrounding smells are perceived by a variety of different receptor molecules that specifically bind certain chemical structures [Buck \& Axel, 1991; Ache \& Young, 2005]. However, the sum of all the chemicals present is not sufficient to "describe" the olfactory environment and an identification of the combination of stimuli is necessary. Therefore, the information of the sum of all activated neurons in response to various molecules is integrated on the second level of the olfactory system. The information is then conveyed to higher brain centers for further processing [Leinwand \& Chalasani, 2011].

This evaluation of the perceived signals can be based on hard-wired neuronal circuits that developed during evolution, but also has to include experiences of each individual and must therefore be subject to plasticity. Thereby, combinations of volatile molecules acting as odorants emitted by a single source can be interpreted by the brain and assigned to this single source to define its smell. Additionally, minor changes of the odor compositions can be compared with previous experiences and help during the evaluation of the new mixture [Wilson \& Stevenson, 2006]. Hence, similar odors can lead to the same behavioral responses. This process of generalization in contrast to discrimination of distinct stimuli can also be found in other sensory systems and is subject of this thesis.

\subsection{Generalization and Discrimination}

The learned association of a sensory stimulus with a reinforcing punishment (negative reinforcement) or reward (positive reinforcement) though classical conditioning has been first described by [Pavlov, 1927]. An animal learns to associate a sensory input (conditioned stimulus, CS) with a reinforcing relevant input (unconditioned stimulus, US) to alter the behavioral response due to a predicted outcome. A behavioral response that is originally elicited by the unconditioned stimulus (unconditioned response, UR), e.g. an approach or an avoidance behavior, will thereby be elicited 


\section{Introduction}

also by the conditioned stimulus (conditioned response, $\mathrm{CR}$ ). During a test for the $\mathrm{CR}$, the CS has to be presented alone in order to distinguish the CR from a UR. As the US is not presented together with CS in the test phase, the learned response is weakened during in the course of the test phase. Several presentations of the CS without the US therefore decreases the CR in an extinction process [Pavlov, 1927]. Pavlov also observed, that a stimulus that is similar to the CS also elicits a CR after associative conditioning and termed this observation "generalization". It was proposed that a strong generalization between two stimuli results from a difficulty to discriminate between them as they are perceptually close together [Lashley \& Wade, 1946]. In contrast, the generalization effect is low for two easily discriminable stimuli [Klein, 2002]. However, the intricacy to discriminate between two stimuli is not the only reason for generalization. In fact, stimuli that can not be discriminated due to equal receptor activation can not be generalized as they are perceived the same. As mentioned above, natural stimuli are not often reoccurring in exactly the same way. It might therefore be beneficial for an individual to actively generalize the response to a stimulus similar to an already experienced and thereby learned one in order to find an appropriate behavioral response [Shepard, 1987].

The process of generalization, in contrast to discrimination, of sensory stimuli was subject of many psychological experiments in the last decades. Guttman \& Kalish [1956] used pigeons to describe a bell-shaped curve of responses to a test stimulus as an effect of generalization. This generalization gradient depended on the similarity of the stimulus presented in the test situation with the conditioned stimulus, in this case light of different wavelengths. They used visual stimulation as the similarity of two stimuli can be easily measured by the wavelength of a light stimulus. Pigeons were trained to respond to an illuminated key with pecking the key in order to receive a food reward. During the generalization testing, the key was illuminated with light in varying wavelengths and the key pecking responses of the pigeons observed. The result showed the above mentioned bell-shaped generalization gradient: wavelengths similar to the trained ones resulted in more key pecking than wavelengths that were more distinct from the CS wavelength [Guttman \& Kalish, 1956; Honig \& Urcuioli, 1981]. 


\section{Introduction}

Similar generalization experiments were also performed with an aversive reinforcer or another stimulus modality (like auditory or tactile) and in different species and resulted in similar generalization gradients. However, the steepness and the width of the gradient may vary depending on the experimental procedures. In experiments were the CS is explicitly associated with no reinforcement, the generalization gradients' bell-shape is turned upside down [Klein, 2002].

A combination of the association of one stimulus (CS+) with a reinforcement and a second stimulus (CS-) with the absence of a reinforcement is called differential conditioning. During the test the individual has to discriminate between the two stimuli in order to predict the reinforcement that is associated with one of the stimuli. During differential conditioning, actually two things are learned: in a conditioned excitation phase, the CS+ is associated with the reinforcement and in a conditioned inhibition phase the CS- is associated with the absence of the reinforcement. As both conditioning phases underly the generalization gradient, the maximal response to linear stimuli (such as visual and auditory stimuli) shifts from the exact CS+ value in the direction away from the CS- [Hearst \& Franklin, 1977; Honig \& Urcuioli, 1981; Klein, 2002].

\subsubsection{Absolute versus differential conditioning}

As mentioned above, differential conditioning can be divided in two distinct phases: the conditioned excitation and the conditioned inhibition. The effect of a conditioned excitor is easily addressed by testing the association of the CS+ with the US for its capability to elicit a CR. On the other hand, the formation of a conditioned inhibition can not be tested directly as the CS- is not explicitly associated with an US and thereby does not elicit any CR. A way to check for conditioned inhibition is the summation effect: When a CS+ elicits a $\mathrm{CR}$, this response should be decreased when the CS+ and the CS- are presented together. Additionally, it should take more conditioning trials to transform a conditioned inhibitor into a CS+ by association with the US than a neutral stimulus [Rescorla, 1969a,b; Savastano et al., 1999]. Experiments in pigeons by Hearst \& Franklin [1977] proposed to include a response opposite to the CR as a measurement for conditioned inhibition. They could show that differential training resulted in an approach towards the stimulus that was positive reinforced 


\section{Introduction}

(CS+) and a withdrawal from the stimulus that was explicitly not reinforced (CS-). An even stronger version of differential conditioning is using a positive reinforcement for the CS+ whereas the CS- is negatively reinforced. Experiments in ants (Camponotus fellah) could show a discrimination between the CS+ and the CS- with dissimilar odorants and could ascribe this effect to a conditioned excitation [Josens et al., 2009].

In contrast to differential conditioning, absolute conditioning does only include one stimulus that is associated with the reinforcement [Giurfa, 2004]. Therefore, absolute conditioning only includes a CS+ and no CS-. The two distinct training paradigms show significant differences in the learning performance in honey bees (Apis melifera) [Giurfa, 2004, 2007]. Even though absolute conditioning of a bee with a visual stimulus as the CS+ (and without a CS-) resulted in a learned CR, a differential conditioning with a reinforced $\mathrm{CS}+$ and a non-reinforced CS- resulted in stronger conditioned responses to the CS+. Additionally, the discrimination between two similar stimuli was increased after differential training whereas absolute training resulted in a decreased discrimination.

\subsubsection{Sensory preconditioning}

Whereas differential conditioning can be used to increase the discrimination between two stimuli, sensory preconditioning represents a phenomenon during which the difference between two stimuli is decreased by the formation of an associative connection between them. When two distinct stimuli (CS1 and CS2) are presented together prior to a conditioning phase in which one of the stimuli (CS1) is associated with a reinforcement (US), the non-trained stimulus (CS2) will also elicit a conditioned response [Pavlov, 1927; Brogden, 1939]. Therefore, the presentation of CS1 and CS2 before the training must have formed an association between the two stimuli so that both stimuli presented alone elicit a response [Brogden, 1939; Kimmel, 1977; Rescorla, 1980].

In the above mentioned experiments, Narbutovich in Pavlovs laboratory, Brogden and Rescorla conducted crossmodal sensory preconditioning in mammals. Crossmodal sensory preconditioning includes stimuli from more than one sensory modality (e.g. visual and auditory stimuli as used by Narbutovich [Pavlov, 1927], Brog- 


\section{Introduction}

den [1939] and Rescorla [1980]). Crossmodal sensory preconditioning with a visual stimulus and an olfactory stimulus during the preconditioning phase has also been investigated in insects. The ability to associate these two modalities during the preconditioning phase could be shown for crickets (Gryllus bimaculatus) |Matsumoto et al., 2013] and in Drosophila [Guo \& Guo, 2005].

Sensory preconditioning was also performed with stimuli of one modality: Brembs \& Heisenberg [2001] used distinct visual stimuli, pattern and color, as CS1 and CS2 and observed a preconditioning effect in Drosophila in a flight simulator. A specific pattern was presented together with a color (CS1 + CS2) and during the conditioning either the color or the pattern was reinforced (CS1). During the test phase, the nonreinforced stimulus (CS2) was tested for a behavioral response. Sensory preconditioning experiments were also performed with exclusively olfactory stimuli. Honey bees (Apis melifera) were stimulated with a binary mixture of two odorous compounds and afterwards conditioned to one of the compounds [Müller et al., 2000]. A subsequent test of the second compound resulted in a conditioned response. Interestingly, a single preconditioning phase with a presentation of the two odorants as a mixture was sufficient to elicit a stronger response to the non-reinforced compound. This effect can be explained by the configural cue theory formulated by Pearce [1994] which suggests a direct coupling of two simultaneously presented stimuli after the first paired presentation. In contrast, the elemental cue theory states that a mixture is perceived as a sum of its elements as introduced by Rescorla \& Wagner [1972]. The elemental model was improved by adding a unique cue that is elicited by the mixture (unique cue theory, [Rescorla, 1973; Miller et al., 1995; Deisig, 2003]).

\subsubsection{Discrimination of olfactory signals}

The ability of individuals to discriminate two sensory stimuli is strongly dependent on the nature of the two stimuli and the experiences the individuals have made. A explained, different associations can be formed depending on simultaneous presentation of the stimuli and the temporal pairing with reinforcing signals. Extensive studies have been performed on behavioral responses of animals to olfactory stimuli and the ability to discriminate them depending on prior conditioning. The ability to 


\section{Introduction}

discriminate between two odorants is also dependent on the concentration in which the odorants are presented: the perceptual quality of the odorant is directly related to the quantity of the odorant. The detection threshold of an olfactory stimulus can, however, be separated from the discrimination threshold. This effect could be shown with natural occuring odors as well as with monomolecular odorants [Wright et al., 2002; Wright, 2004]. It is therefore of importance to use odorant concentrations that are above the discrimination threshold during olfactory conditioning experiments.

In several discrimination experiments monomolecular odorants with a varying length of the carbon chain were used and the similarity between two stimuli quantified with respect to the length of the chain. Indeed, rats showed a correlation between carbon chain length and odorant similarity on the level of three behavioral paradigms (habituation, generalization and discrimination) Cleland et al. [2002]. Olfactory acuity, and thereby the capability to discriminate between two odorants, is dependent on the concentration of the odorant perceived by an individual Cleland \& Narla [2003]. Additionally, conditioning is increasing the olfactory acuity whereas habituation does not [Fletcher \& Wilson, 2002]. The similarity of odorants depending in the length of the carbon chain was also investigated in honey bees. It could be confirmed that odorants with a similar carbon chain length are generalized after absolute conditioning. Another chemical property of monomolecular odorants used during discrimination experiments is the chemical group. Expectedly, differences in odorant similarity can be observed depending on the chemical class of a molecule (primary and secondary alcohols, aldehydes and ketones were considered). The level of generalization on a behavioral level could also be correlated with "activity patterns" evoked by the different odorants in the first processing center of the olfactory system, the olfactory bulb in mice [Rubin \& Katz, 1999] and zebra fish (different amino acids were used as odorants [Friedrich \& Korsching, 1997]), and the antennal lobe in insects (see section 1.4 [Guerrieri et al., 2005].

Alternatively, single odorant compounds and mixtures of these odorants have been used in order to create similar or dissimilar olfactory stimuli and thereby achieve generalization between the mixture and the single compound [Linster \& Smith, 1999]. Varying the concentrations of a binary mixture during either absolute or differential conditioning resulted in two different levels of generalization in honey bees [Wright et al., 2008]. Absolute conditioning with two different concentrations of one odorant 


\section{Introduction}

that were both positively reinforced resulted in a strong generalization of this odorant in all concentrations in the mixture. Differential training on the other hand with one concentration of the odorant positively reinforced and another concentration negatively reinforced led to a clear discrimination of different mixture concentrations in the test[Wright et al., 2008].

However, the perception of odorant mixtures can not be directly compared with the perception of single odorant compounds. Even though the single elements of a mixture are perceived, the compounds are also combined and interact in the olfactory perception process [Deisig, 2003]. Thereby, the mixture is not only perceived as a summation of the two compounds but also as a configural unit as reported for vertebrates [Coureaud et al., 2009] and invertebrates [Silbering \& Galizia, 2007; Honegger et al., 2011].

Therefore, the use of odorant mixtures has been varied: instead of using mixtures and single compounds as similar stimuli, similar odorant mixtures in contrast to dissimilar mixtures were used [Chen et al., 2011; Chapuis \& Wilson, 2011]. It could be shown in rats that an absolute training paradigm results in a strong generalization between two similar odorant mixtures whereas a differential training results in a discrimination [Chen et al., 2011]. Additionally, a neural correlate for this increased discrimination could be found: the size of the "receptive field" in the piriform cortex (the brain region responsible for olfactory information processing in mammals) can perhaps be regarded as a measure for the specificity of an odorant. After differential training, the size of the "receptive field" activated by the olfactory stimulus decreases [Chen et al., 2011]. Similarly, the correlation of the evoked activity patterns decreased after differential conditioning [Chapuis \& Wilson, 2011]. Interestingly, the "receptive field" size increases after absolute conditioning which suggests less olfactory specificity and is in line with the observed stronger generalization.

Conclusively, the discrimination of olfactory signals can be viewed upon from two sides. On one hand, physiological responses of neurons in the first olfactory processing center provide a spatial map of neuronal activity during stimulation. Discrimination of odorants is therefore dependent on the activation pattern elicited by different odorants. Similar odorants evoke overlapping, clustered activation pattern whereas dissimilar odorants elicit distinct patterns [Friedrich \& Korsching, 1997; Rubin \& Katz, 1999; Guerrieri et al., 2005; Friedrich, 2006; Chapuis \& Wilson, 2011]. 


\section{Introduction}

On the other hand, behavioral responses after olfactory conditioning are also providing a measure for olfactory discrimination. The behavior of the animals towards an odorant after conditioning of another odorant is dependent on the similarity of the two odorants. If they are very similar, the behavioral response will be comparable whereas dissimilar odorants evoke distinct responses, if at all [Linster \& Smith, 1999; Wright et al., 2002; Cleland et al., 2002; Wright et al., 2008; Chen et al., 2011].

\subsection{Drosophila melanogaster as a model organism}

The fruit fly Drosophila melanogaster has been used extensively as a model organism to study various biological functions. Since Thomas Hunt Morgan identified the first gene mutation (white, responsible for the eye color of the animals) in 1910, a huge amount of important findings accentuated the fruit fly to be used in genetic research. From the use of balancer chromosomes to prevent uncontrolled recombination of fly crosses (1948, [Greenspan, 2004]) via germline transfection with transgenes ([Rubin \& Spradling, 1982]) and tissue specific expression of transgenes with a binary expression system ([Brand \& Perrimon, 1993], see section 1.3.1] to the sequencing of the whole Drosophila genome ([Adams et al., 2000]) more techniques were developed. Thereby, more research opportunities and potential experiments were possible to be executed [Greenspan, 2004].

Apart from the genetic advantages of Drosophila as a model organism, it is of great importance that the flies posses a much simpler, but to some degree comparable, nervous system than mammals. Even though the Drosophila brain comprises of only $\sim 10^{5}$ neurons (mammals: $10^{8}-10^{11}$ ), various neuronal subclasses and neurotransmitters as well as biogenic amines are shared [Venken et al., 2011]. Additionally, the molecular mechanisms that underly neuronal function, like ion channels and second messenger cascades, are the same in mammals and fruit flies. Most importantly for research in the nervous system and on conditioning mechanisms, the animals are able to perform complex behavioral tasks that include associative learning |Waddell \& Quinn, 2001; Fiala, 2007; Pitman et al., 2009] and even second order conditioning [Tabone \& de Belle, 2011]. The first experiments with olfactory conditioning in Drosophila were performed in 1974 [Quinn et al., 1974] as further described in section 1.5 . 


\section{Introduction}

In the last two decades, several techniques were developed to genetically identify neuronal subclasses and circuits. It is now possible to monitor or even manipulate neuronal activity in specific cells in the living animal in order to investigate the neuronal circuits underlying behavior[White \& Peabody, 2009; Venken et al., 2011].

Cell- or tissue type- specific expression of transgenes is of major importance for the investigation of the function of specific cells or tissues. Binary expression systems are additionally helpful as it is possible to express different transgenic proteins in several cell types without the need to generate new fly strains for each combination of protein and cell type.

\subsubsection{Binary expression systems}

The transformation of Drosophila germline cells in order to generate transgenic fly strains via P-element insertion made it possible to introduce any transgene of choice under a tissue specific promotor [Rubin \& Spradling, 1982]. However, the development of the binary GAL4/UAS-system developed by Brand \& Perrimon [1993] introduced a way to combine the expression of different transgenes in varying cell types without the need to produce new transgenic lines for each combination: the GAL4enhancer, taken from yeast and originally not present in Drosophila, can bind to an upstream activator sequence (UAS) and is thereby promoting the expression of the transgene controlled by UAS (Figure 1.1).

Two different fly strains are generated. The first line is the "driver line" and contains a cell specific regulator sequence controlling the expression of GAL4. Thus, GAL4 is produced only in specific cells. However, GAL4 expression alone does not have any effect. The second line is the "reporter line" containing the gene of interest under the control of the upstream activator sequence (UAS). Every cell contains the UAS region, but the gene of interest is not expressed without GAL4 present to activate the expression. When the driver line and the reporter line are combined, the F1 generation expresses GAL4 in a tissue-specific manner. The GAL4 binds to the UAS and activates the expression of the gene of interest.

In this way, a driver line can be used to induce the cell-specific expression of a variety of genes of interest by combination with different reporter lines. On the other 


\section{Introduction}

hand, a specific gene of interest can be expressed in a variety of specific cell types by the combination with different driver lines [Duffy, 2002].

The GAL4/UAS system could be further improved by the introduction of GAL80 and specifically of GAL80 ${ }^{\text {ts }}$ [Zeidler et al., 2004]. GAL80 in its active form blocks the binding of GAL4 to UAS and therefore suppresses the expression of the gene of interest. A temperature sensitive form of GAL80 can be inactivated by increasing the temperature to $29{ }^{\circ} \mathrm{C}$ and thus induce the expression of the gene of interest at a specific time point [Zeidler et al., 2004]. Conclusively, the GAL4/UAS system provides a powerful tool to induce the expression of a gene of interest in a cell-specific and time-specific manner.

An alternative, independent binary expression system to GAL4/UAS is the LexA/LexOp system [Szüts \& Bienz, 2000; Lai \& Lee, 2006]. Similar to UAS, the expression of a gene of interest is promoted by the regulatory sequence LexOp. Expression only takes place if LexA is binding to LexOp and thereby activates the transcription of the gene of interest. There are two different versions of LexA, a GAL80-sensitive and a GAL80-insensitive version. The GAL80-sensitive version uses a part of the GAL4 protein (GAD) in order to drive the expression of the gene of interest and can therefore be blocked by the expression of GAL80. The insensitive form uses a

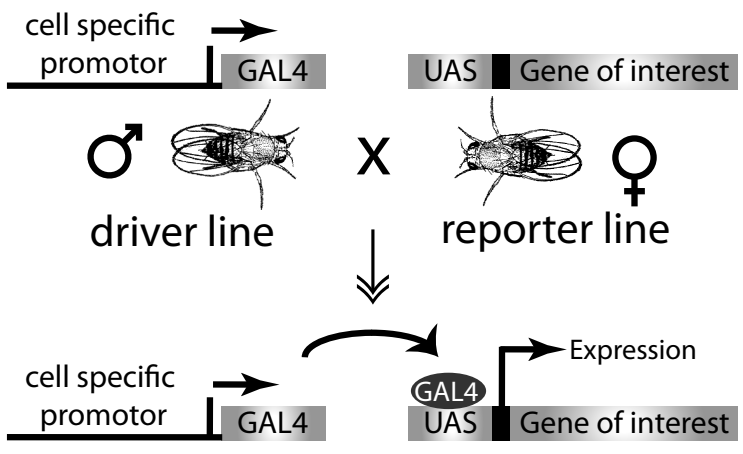

Figure 1.1. The GAL4/UAS system.

The binary GAL4/UAS system is composed of two different fly lines. The driver line expresses GAL4 under a cell specific regulator sequence whereas the reporter line contains the gene of interest under the control of the upstream activator sequence (UAS). Without GAL4 present in the driver line, the gene of interest is not expressed. When the driver and the reporter line are combined, the expression of GAL4 in specific cell types results in a binding of GAL4 to the UAS and in turn activates the expression of the gene of interest.

Picture modified from [Brand \& Perrimon, 1993] viral promotor (VP16) to drive the expression of the gene of interest and can therefore not be blocked by GAL80 [Lai \& Lee, 2006].

Recently, another binary expression system was introduced into Drosophila: the $Q$ system [Potter et al., 2010]. Similar to GAL4/UAS, the expression of the trans- 


\section{Introduction}

gene is controlled by QUAS, whereas QUAS activation is dependent on cell-specific expression of the transcription factor QF. The system also includes an inhibitor, similar to GAL80, QS. When QS is expressed in a cell, it inhibits the binding of QF to QUAS. QS inhibition can be interrupted by feeding of quinic acid (comparable to inactivation of GAL80 ${ }^{\text {ts }}$ via a temperature increase) [Potter et al., 2010].

An advantage of two different and independent binary expression system is the possibility to combine the two systems. It is possible to target two distinct subsets of cell types and express two different genes of interest. Recently, these binary systems have been improved in order to increase the specificity and the expression levels of the genes of interest [Pfeiffer et al., 2010].

The binary expression systems are helpful to target specific cell types as described. By targeting the expression of transgenes to a subset of neurons, it is possible to observe $\mathrm{Ca}^{2+}$-dynamics with calcium indicators as a correlate for neuronal activity [Riemensperger et al., 2012], manipulate the neuronal activity by the expression of ion channels or block the synaptic output by hindering endocytosis [Venken et al., 2011.

\subsubsection{Manipulation of neuronal activity}

The easiest way to manipulate neuronal activity is to artificially change the electrical properties of the cell and therefore hyperpolarize them, i.e. to decrease the membrane potential and thus keep the cells below the firing threshold, or to depolarize them in order to elicit action potentials. Depolarization of the cells can be induced by the expression of different ion channels that conduct sodium or calcium.

The most common mechanisms include temperature or light activated cation channels [Venken et al., 2011]. dTRPA1 is a temperature -ensitive channel that opens upon an increase of the temperature [Hamada et al., 2008]. When expressed in Drosophila neurons, a tonic firing rate of the cells can be observed after an increase of the temperature [Pulver et al., 2009]. Another method to induce depolarization of neurons is via optical stimuli. The most effective light-controlled way to activate neurons is via ATP-uncaging and the expression of the purinoceptive ion channel P2 $\mathrm{X}_{2}$ [Lima \& Miesenböck, 2005; Venken et al., 2011]. Alternatively, the light sensitive ion 


\section{Introduction}

channel channelrhodopsin-2 can be used. Channelrhodopsin-2 is a cation-channel that opens upon stimulation with light with $420 \mathrm{~nm}$ wavelength [Nagel et al., 2003; Boyden et al., 2005; Schroll et al., 2006; [Fiala et al., 2010].

In order to inactivate or silence neuronal activity, the light sensitive chloride pump halorhodopsin can be used [Zhang et al., 2007]. However, as the ion transport of the pump is rather low, a very high expression level of the channel is required for optimal function [Venken et al., 2011].

A more common method to prevent neurons from eliciting action potentials is the use of potassium channels to reduce the resting potential of the cell [Hodge, 2009]. An over-expression of the Kir-channel, an inwardly rectifying potassium channel, increases the potassium conductance and thereby keeps the membrane potential below firing threshold [Baines et al., 2001]. Similarly, the dORK-channel, a voltage and time independent potassium channel, increases the conductance of the neuronal membrane for potassium and therefore lowers the resting membrane potential [Nitabach et al., 2002]. However, the expression of these channels during development alter the maturation of the nervous system and are lethal when expressed pan-neuronally [Hodge, 2009].

Likewise, the expression of tetanus toxin can result in compensatory mechanisms to overcome neuronal circuit dysfunction [Sweeney et al., 1995; Venken et al., 2011]. Tetanus toxin expressed in Drosophila neurons cleaves the synaptic protein synaptobrevin and thereby prevents exocytosis. Synaptobrevin is a part of the SNARE complex that is needed for recruitment of synaptic vesicles to the membrane in order to release neurotransmitters [Fasshauer et al., 1998].

A second and more common method to prevent synaptic transmission is blocking exocytosis in an indirect way. By blocking endocytosis, the vesicle pool for exocytosis is depleted. Blockage of endocytosis is achieved by the expression of a dominant mutant allele of the gene responsible for dynamin expression, shibire [Kosaka \& Ikeda, 1983; Kim \& Wu, 1990; van der Bliek \& Meyerowitz, 1991; Chen et al., 1991]. Dynamin is necessary for endocytosis as it triggers the cleavage of the clathrin coated vesicles from the plasma membrane. Without functional dynamin, the vesicles stick to the membrane and can not be reused and refilled with neurotransmitters [Henley et al., 1999]. The expression of a defective shibire gene therefore prevents synaptic transmission (see Figure 1.2, right side). In 2001, Kitamoto introduced a temper- 


\section{Introduction}

ature sensitive version of shibire to defined neuronal subsets of Drosophila with the GAL4/UAS system. An increase in temperate to $30{ }^{\circ} \mathrm{C}$ resulted in a complete block of synaptic transmission and flies expressing shits in cholinergic neurons paralyzed after a few minutes exposure to the high temperatures. Upon decrease of the temperature back to $22{ }^{\circ} \mathrm{C}$, the flies recovered and started moving again [Kitamoto, 2001]. Hence, it is possible to reversibly block synaptic transmission by changing the temperature (see Figure 1.2). However, the exact temperatures chosen by different research groups as the permissive and the restrictive temperature varies slightly [Venken et al., 2011]. Kitamoto [2001] originally chose $19-22{ }^{\circ} \mathrm{C}$ as permissive and

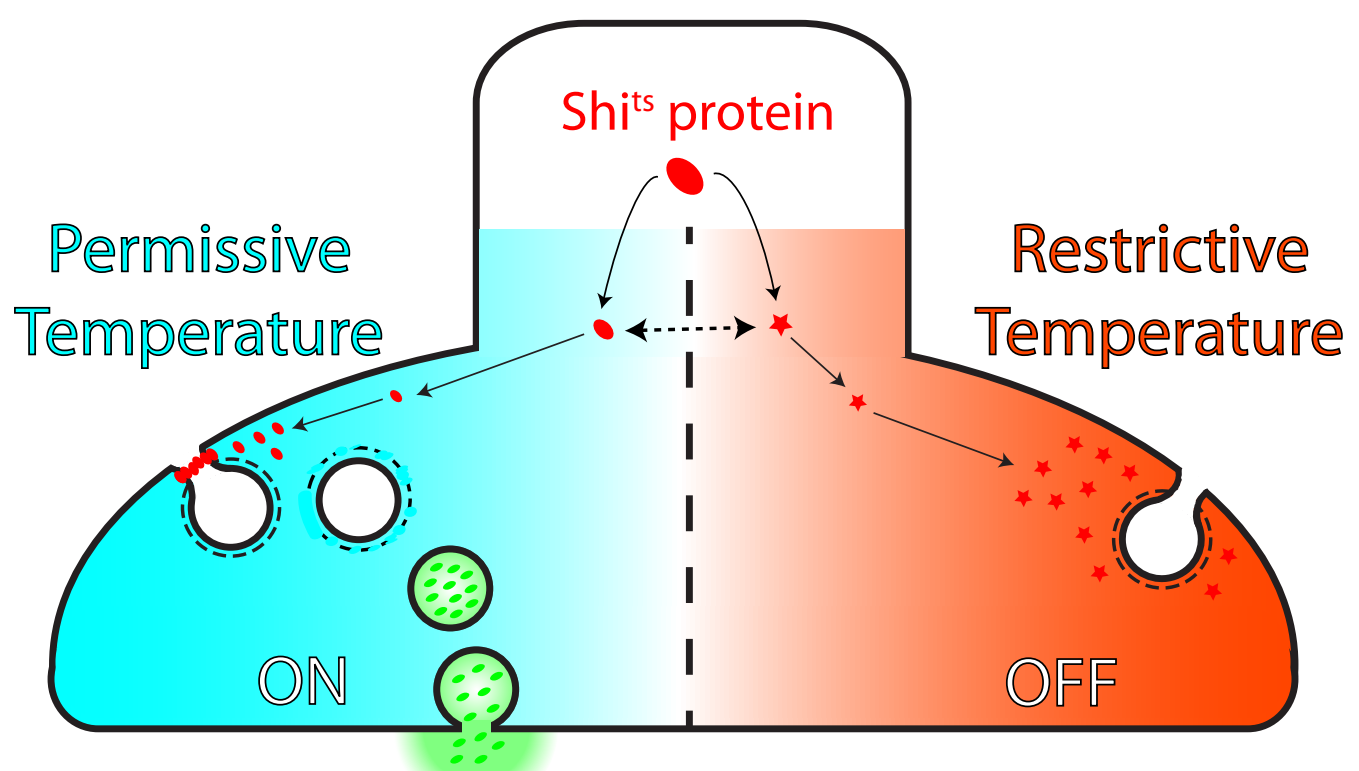

Figure 1.2. Mechanism of shibire ${ }^{\text {ts }}$ function.

The shibire protein is a dynamin homologue and responsible for the cleavage of clathrin coated vesicles from the cell membrane during endocytosis. At the permissive temperature, the protein is functional and endocytosis can take place. After clathrin removal the vesicles can be filled with neurotransmitters. Upon an action potential, the vesicle fuse with the membrane and the neurotransmitters are released (left side). However, at the restrictive temperature, the shibire protein is not functional and endocytosis is blocked. Therefore, the vesicle pool is quickly depleted and exocytosis is inhibited. In turn, no neurotransmitters can be released into the synapse and synaptic transmission is silenced. After a decrease of the temperature back to the permissive level, the protein recovers and becomes fully functional again.

Picture modified from [Kasuya, 2009] 


\section{Introduction}

$30{ }^{\circ} \mathrm{C}$ as restrictive temperature, whereas Schwaerzel et al. used $26{ }^{\circ} \mathrm{C}$ and $34{ }^{\circ} \mathrm{C}$, respectively [Schwaerzel et al., 2002, 2003]. A permissive temperature of $25^{\circ} \mathrm{C}$ is of advantage as the flies can be raised and kept in incubators at $25^{\circ} \mathrm{C}$ and handled in the laboratory without keeping the temperature constantly low.

The block of synaptic transmission via shibire ${ }^{\text {ts }}$ is broadly used in Drosophila learning an memory research in order to investigate the function of several neuronal subclasses during the different steps of memory formation [Schwaerzel et al., 2003; Akalal et al., 2006; Keene et al., 2006; Krashes et al., 2007; Aso et al., 2010; Trannoy et al., 2011; Chen et al., 2012; Liu et al., 2012. With the help of shibire ${ }^{\text {ts }}$, neuronal silencing can be timed to the phases of formation, consolidation or retrieval of memory [Kasuya, 2009]. In the present study, local circuits in the antennal lobe have been interrupted with shibire ${ }^{\text {ts }}$. Thereby, the functional involvement in generalization and discrimination tasks was investigated.

In addition to manipulate neuronal activity, it is of great interest to monitor neuronal activity during different tasks and following various stimuli.

\subsubsection{Monitoring of neuronal activity using in-vivo calcium imaging}

Monitoring and recording of action potentials is a powerful tool to investigate the functions of neuronal circuits and the responses of neurons to diverse stimuli. As electrophysiological recordings are limited to single cells or small populations of neurons surrounding an extracellular electrode, non-invasive optical imaging methods provide certain advantages with respect to monitoring the activity of multiple cells simultaneously [Griesbeck, 2004]. Since the first optical method to visualize neuronal activity in sensory cells with voltage sensitive dyes [Salzberg et al., 1973], the techniques used for optical imaging significantly increased. One approach was to visualize the calcium ion influx into a neuron upon an action potential [Stallcup, 1979]. The increased $\mathrm{Ca}^{2+}$ concentration in the cytoplasm that can be measured after neuronal activity is due to an influx of calcium ions from the extracellular space and an efflux from the lumen of the endoplasmatic reticulum [Berridge, 1998].

Several disadvantages of optical calcium measurements have to be addressed. It is hardly possible to visualize inhibitory activity with the help of calcium dyes and the 


\section{Introduction}

dynamic range of the sensor was initially very limited. Thus, the temporal resolution of the method is rather low compared to electrophysiological methods. Additionally, the targeting of the sensors to specific areas or cell types was difficult even though a great variety of synthetic dyes existed in order to visualize changes in $\mathrm{Ca}^{2+}$ concentrations [Knöpfel et al., 2006]. Therefore, genetically encoded calcium sensors provided an excellent tool to overcome some of these problems. The expression of the sensors can be specifically targeted to an area or cell type of interest and binary expression systems can be used to monitor several neuronal subtypes in Drosophila [Griesbeck, 2004; Fiala et al., 2002]. The variety of genetically encoded calcium sensors (GECls) greatly increased in the last years and the (initially poor) temporal resolution could be significantly improved [Koldenkova \& Nagai, 2013].

\subsubsection{Genetically encoded calcium indicators (GECls)}

The first genetically encoded calcium sensors were developed in 1997 [Miyawaki et al., 1997]. The sensor, named "Cameleon", consisted of two fluorescent proteins (enhanced cyan, ECFP, and enhanced yellow fluorescent protein, EYFP) that were connected via a calmodulin and a M13 domain. Illumination of the complex with light in a wavelength of $\sim 440 \mathrm{~nm}$ in the absence of calcium ions results in an excitation of ECFP and an emission of light with a wavelength of $\sim 480 \mathrm{~nm}$. In the presence of $\mathrm{Ca}^{2+}$, the ions bind to calmodulin that in turn binds to $\mathrm{M} 13$ and therefore changes the conformation of the protein and brings the ECFP and the EYFP domains in closer spatial proximity. This decreased distance promotes an transfer of the energy from the ECFP to the EYFP via Förster resonance energy transfer (FRET). An excitation of ECFP with $\sim 440 \mathrm{~nm}$ leads thereby to a transfer of energy to ECFP and an excitation of light with $\sim 535 \mathrm{~nm}$ (Figure $1.3 \mathrm{~A}$ ). The ratio of light emitted with 480 and $535 \mathrm{~nm}$ is therefore a measurement of calcium ion concentration [Miyawaki et al., 1997).

In the following years, different versions of ratiometric calcium sensors with varying properties have been developed. The wavelength of excitation light and emission light could be altered by an exchange of the fluorescent proteins and the $\mathrm{Ca}^{2+}$ affinity and the dynamic range of the sensors could be increased by a mutation of the protein residues or an exchange of the connective domains [Guerrero \& Isacoff, 2001; Griesbeck, 2004; Knöpfel et al., 2006; Koldenkova \& Nagai, 2013]. Measure- 
A
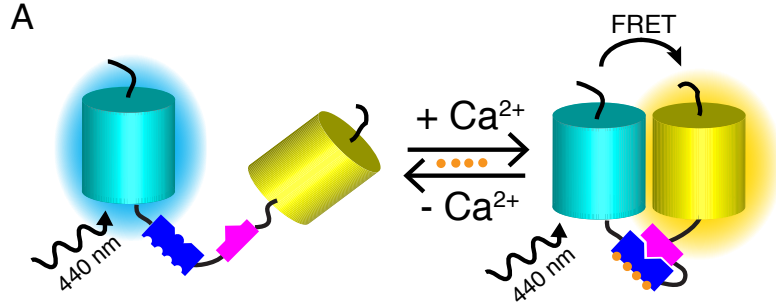

B

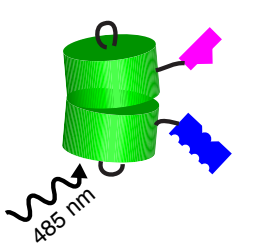

C

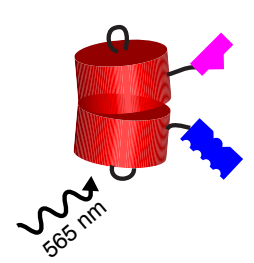

M13
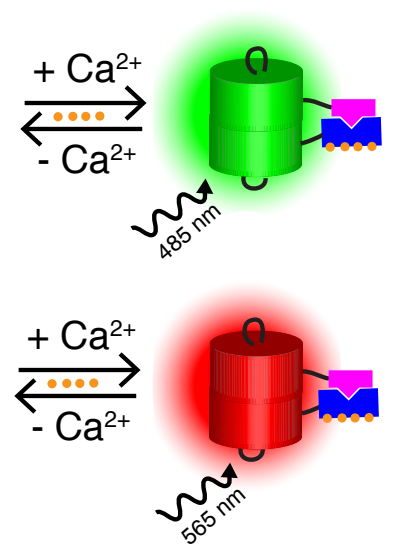

Calmodulin
Figure 1.3. Schematics of different GECls.

Genetically encoded calcium sensors are subdivided in two groups. Ratiometric metric sensors consist of two fluorescing proteins and change the wavelength of the emitted light upon calcium ion binding whereas single protein sensors only emit light when $\mathrm{Ca}^{2+}$ is present. (A) Yellow Cameleon as an example for ratiometric sensors emits blue light when the ECFP (light blue) is excited and no calcium ions are bound to the calmodulin (blue) domain. Upon $\mathrm{Ca}^{2+}$ binding (orange), calmodulin changes its conformation and the M13 domain (magenta) of the protein can bind. Due to this change, the EYFP

(yellow) get in closer proximity to the ECFP and an energy transfer can take place. Thus, the emitted light shifts from blue to yellow upon $\mathrm{Ca}^{2+}$ binding. (B) GCaMP on the other hand is a single protein sensor. It consists of a circular permutated GFP (green) with a calmodulin at its $\mathrm{C}$ terminus and a M13 domain at the $\mathrm{N}$ terminus. Upon $\mathrm{Ca}^{2+}$ binding to calmodulin, $\mathrm{M} 13$ can bind and the conformational change in the protein increases the emitted light upon excitation. (C) R-GECO, a red shifted variant of GCaMP (red), has a similar functionality. The emitted light upon excitation increases with $\mathrm{Ca}^{2+}$ binding.

Picture modified from [Koldenkova \& Nagai, 2013]

ments of neuronal activity with ratiometric calcium sensors in Drosophila were first performed in 2002 by Fiala et al. who measured the response of olfactory sensory neurons to olfactory stimulation with odorants with the sensor Yellow Cameleon 2.1 [Fiala et al., 2002].

In contrast to the ratiometric sensors, non-ratiometric sensors consist of only one fluorescent protein [Koldenkova \& Nagai, 2013]. The strongest impact on research with $\mathrm{Ca}^{2+}$-imaging had the development of GCaMP in 2001 [Nakai et al., 2001]. A circular permutation of GFP resulted in higher fluorescence levels of the protein whereas the addition of calmodulin to the $\mathrm{C}$ terminus and $\mathrm{M} 13$ to the $\mathrm{N}$ terminus added a $\mathrm{Ca}^{2+}$ sensitivity. The fluorescence of the protein in absence of calcium ions 


\section{Introduction}

is rather low whereas binding of the ion to calmodulin and the resulting connection of calmodulin with M13 increases the fluorescence by a conformational change of the GFP barrel (Figure 1.3 B) [Nakai et al., 2001]. The development of several improved versions of GCaMP added the possibility to measure in neuronal subregions (GCaMP1.6 [Ohkura et al., 2005]) and increased the sensitivity to visualize changes evoked by single action potentials (GCaMP3.0 [Tian et al., 2009]).

Recently, additional variations of GCaMP3.0 resulted in the generation of new genetically encoded calcium sensors in different colors. The excitation and emission wavelengths of GCaMP could be shifted from green ( $485 \mathrm{~nm} ; 515 \mathrm{~nm}$ ) to red (565 $\mathrm{nm} ; 600 \mathrm{~nm}$ ) or blue ( $375 \mathrm{~nm} ; 445 \mathrm{~nm}$ ) [Zhao et al., 2011] (Figure 1.3 C). The development of red and blue fluorescent single protein calcium sensors opens the door for multicolor imaging and combination of different colored sensors in order to measure the calcium activity in subcompartments of cells or different neuronal subpopulations in the same organism [Lindenburg \& Merkx, 2012].

The above mentioned sensors include only a small subset of the broad range of different $\mathrm{Ca}^{2+}$ probes available for research today. Depending on the specifications of the experiment, different sensors with various dynamic ranges, dissociation constants and $\mathrm{Ca}^{2+}$ affinities can be found. The description did not include bioluminiscent sensors that do not need an excitation but a light-emitting cofactor such as BRAC [Saito et al., 2010] and Nano-lantern $\left(\mathrm{Ca}^{2+}\right)$ [Saito et al., 2012] or GFPaequorin [Baubet et al., 2000]. Additionally, the single protein sensors Pericam [Nagai et al., 2001], Camgaroo [Baird et al., 1999], CatchER [Tang et al., 2011] and their subsequent improvements were not mentioned. The same counts for the ratiometric TN sensor and its derivatives where the calmodulin and the M13 domain was replaced by Troponin C [Heim \& Griesbeck, 2004]. A broad overview about existing calcium sensors and the evaluation of their properties can be found in a recent review by Koldenkova \& Nagai [2013].

Conclusively, the various genetic possibilities of Drosophila including the specific targeting of cellular subclasses, the manipulation of neuronal circuits [Venken et al., 2011] and the monitoring of neuronal activity [Riemensperger et al., 2012] prime the animal for the use as a model organism in neuroscience. A large amount of experiments have been performed in the fruit fly in order to shed light on the olfactory system and learning and memory in insects. 


\section{Introduction}

\subsection{The olfactory system of the fly}

The sensation of volatile chemicals is very conserved among species [Ache \& Young, 2005]. As described above, the olfactory sense is very important in order to perceive the environment and possible threads or beneficial situations for an individual. Since the discovery of the olfactory receptor gene family in mammals by Buck \& Axel [1991], many details on the different levels of the olfactory system could be identified. Despite the work done on vertebrates, insects have been a great source of information. The molecular mechanisms of activation of olfactory sensory neurons in the primary olfactory organs have been characterized and the information processing along the olfactory pathway investigated [reviewed by Leinwand \& Chalasani, 2011; Vosshall \& Stocker, 2007]. In the following paragraphs, the olfactory system of Drosophila will be explained on an anatomical and functional basis. However, plasticity of the system during learning processes will be described in more detail in section 1.5 .

\subsubsection{Peripheral detection of odorants at the sensory organ}

The first step in the perception of volatile chemicals is the detection of odorants in the peripheral olfactory organs by sensory neurons. WThese neurons are located in the olfactory epithelium in vertebrates, whereas olfactory sensory neurons in Drosophila are situated on the third segment of the antennae and the maxillary palps [Su et al., 2009]. The 1,200 neurons per antenna [Stocker et al., 1990] are localized in hairlike structures, the olfactory sensilla [Shanbhag et al., 1999; de Bruyne et al., 2001]. They are subdivided into three major classes based on their morphological structure and the responsiveness to subgroups of chemicals. Basiconic sensillae are long and thick, contain two to four neurons and respond to food odorants. Coeloconic sensillae are thick and small and are innervated by two to three neurons which are activated mainly by food odors and humidity levels. Tricoid sensillae respond poorly to most odorants but are highly sensitive to pheromones. They are long and thin and house up to three neurons. All major classes of sensillae are present on the antennae, whereas the maxillary palps contain only the basiconic type [Shanbhag et al., 1999; de Bruyne et al., 2001; Vosshall \& Stocker, 2007; Ronderos \& Smith, 2009]. 


\section{Introduction}

The olfactory sensory neurons (OSNs) in the sensillae are activated by volatile chemicals via olfactory receptors (ORs) [Gao \& Chess, 1999; Clyne et al., 1999; Vosshall et al., 1999|. Each OSN usually expresses one type of receptor which dimerizes with the olfactory co-receptor, ORCO or Or83b in Drosophila [Elmore et al., 2003; Larsson et al., 2004; Neuhaus et al., 2005; Benton et al., 2006; Vosshall \& Stocker, 2007; Ronderos \& Smith, 2009; Nakagawa \& Vosshall, 2009]. Recent studies proposed the ligand binding to the olfactory receptor, which in turn interacts with Or83b. The exact transduction complex is currently under debate. Or83b is suggested to either form an a heteromeric ion channel together with the OR [Sato et al., 2008] or to form the ion-channel itself [Wicher et al., 2008] and thereby mediating the signal transduction. Additionally, Or83b is suggested to modulate the response to an odor via the cAMP signaling cascade (Figure 1.4 B) [Wicher et al., 2008; Nakagawa \& Vosshall, 2009].

Whereas the tricoid sensillea are responding to pheromones and expressing Or83b, only one OR could be identified in coeloconic sensilla [Su et al., 2009]. Recently, another type of olfactory receptors could be identified, the so called ionotropic receptors (IRs) [Benton et al., 2009]. They are located in coeloconic sensillae and have a similar structure than ionotropic glutamate receptors. IRs are ion-channels that are directly activated by ligand binding and do not co-express Or83b [Benton et al., 2009]. Basiconic sensillae, on the other hand, mainly express Or83b and respond to food odors. An exception is the detection of $\mathrm{CO}_{2}$ : the receptors responsive to the gas are from the gustatory gene family [de Bruyne et al., 2001; Larsson et al., 2004; Suh et al., 2004; Kwon et al., 2007; Vosshall \& Stocker, 2007; Su et al., 2009; Ronderos \& Smith, 2009].

The different olfactory receptors have been investigated in detail with regard to their ligands. The "empty neuron" system comprises of an olfactory neuron that is lacking its original OR [Dobritsa et al., 2003]. The expression of different transgenic ORs in the neuron made it possible to identify the ligands binding to this specific OR [e.g. Hallem et al., 2004; Fishilevich \& Vosshall, 2005; Couto et al., 2005; Hallem \& Carlson, 2006]. Galizia et al. [2010] are summarizing these data in the Database of Odorant Responses (DoOR) available online at http://neuro.uni-konstanz.de/DoOR. 


\section{Introduction}

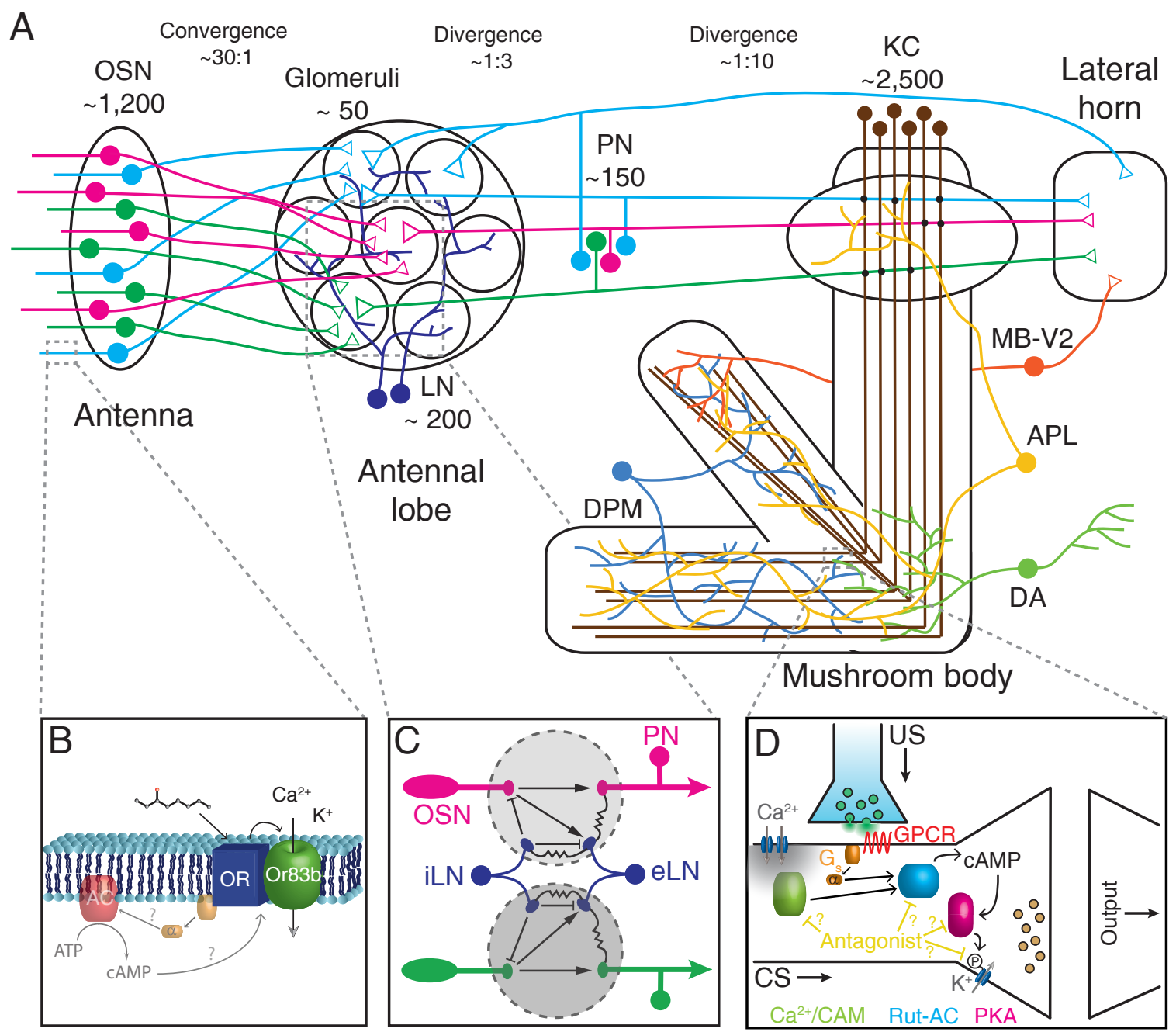

Figure 1.4. The olfactory system of the fly.

(A) Chemical signals are sensed by the olfactory sensory neurons (OSN) in the antenna. OSNs that express the same receptor converge to the same glomerulus in the first processing center, the antennal lobe. Local interneurons (LNs) preprocess the olfactory information before projection neurons (PNs) convey the signal to higher brain centers, namely the mushroom body and the lateral horn. Whereas most projection neurons synapse in the calyx of the mushroom body before they terminate in the lateral horn, some neurons bypass the mushroom body and project directly to the lateral horn. The PNs synapse with mushroom body intrinsic neurons, the Kenyon cells (KCs) at the calyx. A sparse encoding of odor information in the KCs is generated by the innervation of single KCs by distinct PNs. Distinct subclasses of KCs innervate 


\section{Introduction}

different lobes of the mushroom body. Whereas the $\gamma$-KCs form the horizontal $\gamma$-lobes, $\alpha / \beta$ - and $\alpha^{\prime} / \beta^{\prime}$-neurons bifurcate and form the vertical $\alpha / \alpha^{\prime}$ - and the horizontal $\beta / \beta$ 'lobes. Association of olfactory information with a reinforcement signal mediated by dopaminergic neurons (DA) is computed by coincidence detection in the KCs. Dorsal paired medial (DPM) and anterior paired lateral (APL) neurons further process the association signal and the MB-V2 neurons constitute an output from the vertical lobes to the lateral horn. (B) Olfactory receptors (OR, blue) dimerize with the olfactory coreceptor Or83b (green). Upon ligand binding to the OR, the co-receptor acts as a cation channel. A possible function of the receptor dimer in activation of a G-protein coupled messenger cascade involving an adenylate cyclase (AC, red) and an increase in cAMP-levels to modulate the neuronal response is under debate. (C) The antennal lobe network includes OSN (input), PNs (output) and LNs (processing of input and output). LNs can be subdivided into excitatory (eLN) and inhibitory LNs (ILN). Whereas OSNs are excitatory connected to eLNs and PNs, the GABAergic iLNs inhibit OSNs and eLNs. eLNs on the other hand are electrically coupled with PNs and iLNs. (D) Coincidence detection of a reinforcement signal (unconditioned stimulus, US) with an olfactory input (conditioned stimulus, CS) in a Kenyon cell. A depolarization of the KC via the CS leads to a calcium influx and thereby to an activation of calmodulin (green) whereas G-protein coupled receptor (GPCR) binding from the US-signaling activates the $\alpha$ - subunit of the G-protein. Both signals act on the adenylat cyclase rutabaga (light blue) which in turn increases the cAMP level. Increased cAMP levels activate protein kinase A (PKA, magenta) which phosphorylates target proteins such as potassium channels to manipulate the conductance.

Figure modified from |Masse et al., 2009; Ronderos \& Smith, 2009; Tootoonian \& Laurent, 2010; Schwaerzel et al., 2002]

The olfactory sensory neurons project from the antennae and the maxillary palps to the first processing center of the olfactory system, the antennal lobe. In the antennal lobe, the OSNs that express one class of olfactory receptor converge into glomeruli. It could be confirmed that in most, but not all, cases each glomerulus is innervated by only one class of OSNs [Gao et al., 2000; Vosshall et al., 2000; Hallem et al., 2004; Couto et al., 2005; Goldman et al., 2005]. The total number of glomeruli varies in the literature, but $\sim 50$ glomeruli could be identified in the antennal lobe of adult Drosophila [Laissue et al., 1999; Stocker, 2001; Marin et al., 2002; Hallem et al., 2004; Fishilevich \& Vosshall, 2005; Couto et al., 2005; Masse et al., 2009; Seki et al., 2010]. With the help of functional imaging, the identity of the glomeruli was investigated and a conserved stereotypic map of odor activation across individuals could be found Galizia et al., 1999; Fiala et al., 2002; $\mathrm{Ng}$ et al., 


\section{Introduction}

2002; Wang et al., 2003a; Sachse et al., 2007]. In the different glomeruli, the olfactory information is processed via the antennal lobe network and projection neurons (PNs) which in turn convey the signal to higher processing centers in the brain, namely the mushroom body and the lateral horn (Figure $1.4 \mathrm{~A}$ ).

\subsubsection{Primary processing of olfactory information in the antennal lobe}

The first synapses of the olfactory system are located in the antennal lobes (AL) where the axonal projections of the OSNs form glomerular structures and connect to projection neurons that further convey the olfactory information [see Vosshall \& Stocker, 2007, for a review]. Most axonal branches of OSNs target the ipsilateral as well as the contralateral side of the antennal lobe [Stocker et al., 1990]. A strong convergence of information ( 25:1) takes place as $\sim 1,200$ OSNs connect to $\sim 50$ glomeruli [Stocker et al., 1990; Laissue et al., 1999; Stocker, 2001; Hallem et al., 2004; Fishilevich \& Vosshall, 2005; Couto et al., 2005; Masse et al., 2009; Seki et al., 2010]. The glomeruli in turn are innervated by $\sim 150$ PNs per hemisphere which describes a divergence of 1:3 [Stocker et al., 1997; Jefferis et al., 2001; Marin et al., 2002]. Additionally, 200 local interneurons (LNs) in the antennal lobe provide a first processing center of olfactory information and can modulate the output to the projection neurons [Stocker et al., 1997; $\mathrm{Ng}$ et al., 2002; Olsen \& Wilson, 2008; Masse et al., 2009; Seki et al., 2010; Tanaka et al., 2012]. The local interneurons are divided into two main subclasses: excitatory and inhibitory interneurons (eLN and iLN). Further subdivisions by morphology and functional properties revealed distinct subgroups named type I (LN1, labeled by the GAL4-enhancer-trap line NP1227) and type II (LN2, labeled by the GAL4-enhancer-trap line NP2426) inhibitory local interneurons and Krasavietz-positive interneurons (labeled by the Krasavietz-GAL4 line). The Krasavietz class can be subdivided again in excitatory and inhibitory class I and class II neurons [ $\mathrm{Ng}$ et al., 2002; Sachse et al., 2007; Shang et al., 2007; Olsen \& Wilson, 2008; Okada et al., 2009; Seki et al., 2010; Chou et al., 2010; Huang et al., 2010; Yaksi \& Wilson, 2010].

The network of local interneuron connections in the antennal lobe is very dense. Excitatory LNs have been shown to form electrical synapses with PNs and inhibitory 


\section{Introduction}

LNs in the antennal lobe in addition to cholinergic chemical synapses [Tootoonian \& Laurent, 2010; Huang et al., 2010; Yaksi \& Wilson, 2010]. However, most of the local interneurons in the antennal lobe are GABAergic and therefore inhibitory. About half of the iLNs are only innervating the ipsilateral $A L$, whereas the other half project its arborizations also to the contralateral side and acts bilaterally. Most inhibitory interneurons are innervating several glomeruli (multiglomerular iLNs) and only few of them are restricted to a subset of glomeruli (oligoglomerular iLNs) [Chou et al., 2010; Tanaka et al., 2012]. The multiglomerular distribution of iLNs suggests a broad inhibition of several glomeruli by the activity of one interneuron [Chou et al., 2010; Seki et al., 2010; Tanaka et al., 2012]. However, recent research in the honey bee suggests a "patchy" distribution of oligoglomerular inhibitory interneurons in order to locally inhibit specific glomeruli in a distance independent manner Girardin et al., 2013. The complex network of antennal lobe neurons and their connections are depicted in Figure $1.4 \mathrm{C}$.

A more detailed analysis of type I and type II local interneurons (LN1 and LN2) revealed major differences in their morphology. Whereas LN2 neurons form arborizations in glomerular subregions that are innervated by OSNs, LN1 neurons avoid these regions and only synapse with PNs [Okada et al., 2009; Tanaka et al., 2009, 2012]. The function of the antennal lobe network during odor processing has been subject to further research. Optical imaging as well as electrophysiological methods have been used in the honey bee and the fruit fly to shed light on the complex network described above [Wilson, 2004; Ng et al., 2002; Sachse \& Galizia, 2002]. Responses of OSNs and PNs in the antennal lobe to olfactory stimulation are stereotypic among individuals and can be mapped to glomerular activation patterns in the $\mathrm{AL}$. With this stereotypic presentation of odor evoked activation, the response of a single glomerulus to an odor stimulation can be predicted on the level of single OSNs and PNs. If an OSN is responding to an odor stimulus, the PNs innervating the respective glomerulus will respond as well. The synapses between OSNs and PNs have been shown to be very strong which results in a PN activation even if the input to OSNs is low Kazama \& Wilson, 2008. Additionally, the high convergence from OSNs to PNs increases the reliability of PN output and thereby the signal-to-noise-ratio [Bhandawat et al., 2007; Masse et al., 2009]. Local interneurons shape the output of the antennal lobe via lateral inhibition and deinhibition as well 


\section{Introduction}

as excitation. Additional PNs can be recruited in response to an odor stimulation depending on the odor concentration and odor identity [Wilson, 2004; Bhandawat et al., 2007]. Similarly, the interneurons can cause an inhibition of glomeruli [Wilson, 2005|. Thereby, the modulation of an olfactory signal can result in an increased response in PNs for weak stimuli whereas inhibition of strong responses prevents the saturation of the system [Bhandawat et al., 2007; Masse et al., 2009]. Hence, the glomeruli act as individual amplifiers with a varying gain dependent on the olfactory input [Masse et al., 2009]. As PNs respond more reliably to olfactory stimulation, odor identity can be coded in a combination of activated PNs as a response to the signals evoked in OSNs [Masse et al., 2009; Liang \& Luo, 2010; Girardin et al., 2013]. Another evidence for the function of the antennal lobe network in fine tuning of odor responses could be found by a combination of optical imaging and behavioral data. The possibility to discriminate two odorants after olfactory conditioning reflected the similarity of odor evoked patterns in the projection neurons but not the olfactory sensory neurons [Niewalda et al., 2011].

A more complex approach to study the function of the antennal lobe network is the response to odor mixtures. Whereas the activity patterns observed in OSNs due to stimulation with binary mixtures reflected a summation of the components of the mixture, responses of PNs were influenced by the antennal lobe network in response to the mixture. The single component responses were suppressed and a unique activation pattern for the mixture could be observed [Deisig et al., 2006; Silbering \& Galizia, 2007]. These findings speak in favor of the antennal lobe as a first functional unit for both separation and categorizing olfactory information. The broadening of olfactory input increases the response of projection neurons to a weak activation of OSNs whereas multiglomerular inhibition prevents a saturation of the network in response to strong activation [Masse et al., 2009].

The modified output of the antennal lobe as the first processing neuropil of the olfactory system in insects is conveyed via the projection neurons to higher brain centers. The axonal projections of PNs target the mushroom body and the lateral horn. Whereas the mushroom body was shown to be the structure underlying the association of olfactory information with reinforcement during conditioning, the lateral horn was proposed to be involved in the innate repulsion of high concentrations of olfactory stimuli [de Belle \& Heisenberg, 1994; Marin et al., 2002; Wang et al., 2003b]. 


\section{Introduction}

\subsubsection{Sparse coding of odors in the mushroom body}

Most projection neurons convey the olfactory information to the mushroom body (MB) calyx and terminate in the lateral horn (LH) via the inner antennocerebral tract. Another subgroup of projection neurons does not innervate the MB calyx and directly send projections to the lateral horn via the middle and the outer antennocerebral tract [Yasuyama et al., 2003]. The axonal connections in the lateral horn are highly stereotypic between individual flies and are therefore hard-wired [Lin et al., 2007; Tanaka et al., 2004, 2012]. Hence, the axonal projection of a PN terminating in the $\mathrm{LH}$ allow a prediction of the glomerulus from which this PN receives its input [Marin et al., 2002]. Overlapping innervation patterns of single PNs might suggest a combinatorial map in the LH that can be responsible for odor identification and the translation of the input in an appropriate output [Stocker et al., 1990; Marin et al., 2002; Yasuyama et al., 2003; Wang et al., 2003b; Tanaka et al., 2008]. The direct translation of olfactory input to behavioral output without an influence of prior experiences is considered the innate response of the animal. As an ablation of the mushroom body mainly abolished the ability of flies to associate a negative reinforcement with an olfactory input [de Belle \& Heisenberg, 1994] but not the innate avoidance of high odorant concentrations, the lateral horn was proposed to be responsible for the innate olfactory response of aversive stimuli [Wang et al., 2003b]. Whereas the function of the mushroom body in associative learning on a systems level will be described in section 1.5 , its anatomical features (including related neurons) and biochemical reactions to olfactory conditioning will be described here.

The mushroom body consists of $\sim 2,000-2,500$ Kenyon cells $(\mathrm{KC})$ per hemisphere [Technau \& Heisenberg, 1982; Aso et al., 2009] and can be subdivided in three main regions: the calyx, the peduncle and the lobes. The cell bodies of the KCs are clustered and send out dendritic branches to form the calyx as the input area of the MB. The bundled projections of this dendritic tree form the peduncle before they arborize into the lobes. The lobes can be subdivided into the vertical $\alpha$ - and $\alpha$ '- lobes and the horizontal $\beta$-, $\beta^{\prime}$ - and $\gamma$-lobes (Figure 1.5] [Crittenden et al., 1998]. The Kenyon cells can be classified into three major classes: whereas the $\gamma$-neurons (33\% of all KCs) only form the horizontal $\gamma$-lobe, the $\alpha / \beta-(49 \%)$ and $\alpha^{\prime} / \beta^{\prime}$-neurons (18\%) bifurcate to form the vertical $\alpha / \alpha$ ' and the horizontal $\beta / \beta$ '- lobes [Aso et al., 2009]. All three types of KCs arborize broadly in the calyx and could therefore potentially receive olfactory 


\section{Introduction}

Figure 1.5. 3D-model of the mushroom body lobes.

The image of the reconstruction of the mushroom body was taken and slightly modified from [Tanaka et al., 2008]. The cell bodies of the Kenyon cells (light gray) are situated in the posterior cortex and project their axons via the pedunculus (dark gray) to the horizontal (beige) and vertical (blue) lobes. Kenyon cell processes form the calyx (dark gray) as the input region of the mushroom body. Axonal projections of $\alpha^{\prime} / \beta^{\prime}-$ and $\alpha / \beta$-neurons bifurcate to innervate the vertical $\left(\alpha / \alpha^{\prime}\right)$ and the horizonal $\left(\beta / \beta^{\prime}\right)$ lobes whereas $\gamma$-neurons only arborize in the $\gamma$-region of the horizontal lobe.

$\mathrm{D}=$ dorsal; $\mathrm{P}=$ posterior $; \quad \mathrm{M}=$ medial Image taken from [Tanaka et al., 2008]

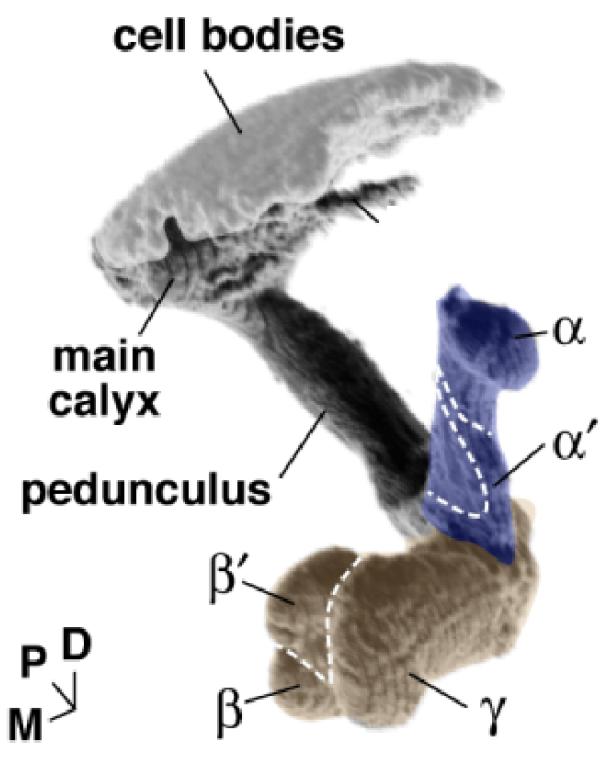

information. The innervation of the calyx by projection neurons from the antennal lobe was shown to be stereotypic [Lin et al., 2007; Tanaka et al., 2004; Leiss et al., 2009; Tanaka et al., 2012]. Therefore, the laminar structure of the stereotypic map might reflect a sorted input of functionally related olfactory information [Lin et al., 2007; Tanaka et al., 2008].

In contrast to the stereotypic map that can be found in the PNs innervating in the calyx, the KCs itself lack this stereotypic feature which suggest variable and plastic connections [Murthy et al., 2008; Honegger et al., 2011]. This was supported by the discovery of actin-rich regions in the dendritic extensions which connect KCs and PNs: they synapse in microglomeruli where several KCs extend claw-like extensions onto large cholinergic boutons of the PNs [Yasuyama et al., 2002; Leiss et al., 2009; Groh \& Rössler, 2011; Butcher et al., 2012]. These microglomeruli are additionally innervated by GABAergic neurons which proposes inhibitory modulation of olfactory input to the mushroom body at the calyx. The plasticity of the microglomeruli in the calyx could be directly connected to the activity of the innervating projection neurons in Drosophila [Kremer et al., 2010]. A caste specific plasticity of the microglomeruli could also be shown in honey bees [Groh et al., 2006]. Despite a smaller number of microglomeruli in queens when compared to worker bees, the rearing temperature and the age of queens influences the number of microglomeruli in queens. 


\section{Introduction}

The dorsal paired medial (DPM) neurons are an example of other identified intrinsic mushroom body neurons (= neurons that only arborize within the MB). They form arborizations exclusively in the horizontal and vertical lobes. An expression of the amnesiac gene and serotonergic transmission in DPM neurons is necessary during olfactory consolidation of memory [Waddell et al., 2000; Yu et al., 2005; Keene et al., 2004, 2006; Lee et al., 2011]. Another neuron that falls into the category of intrinsic mushroom body neurons related to olfactory memory are the GABAergic anterior paired lateral (APL) neurons [Tanaka et al., 2008; Liu \& Davis, 2009; Busto et al., 2010; Wu et al., 2011; Pitman et al., 2011]. APL neurons extend processes to the vertical and horizontal lobes as well as to the calyx. The electric coupling between APL and DPM neurons via heterotypic gap junctions is crucial for olfactory memory formation [Wu et al., 2011].

The divergence of $\sim 150$ PNs onto $\sim 2,500 \mathrm{KCs}$ results in a sparse combinatorial map that is variable across individuals but shows strongly correlated responses to the same odor in one individual [Perez-Orive, 2002; Wang et al., 2004; Szyszka et al., 2005; Turner et al., 2008; Galizia \& Szyszka, 2008; Honegger et al., 2011]. Whereas more than $50 \%$ of PNs respond to a single odor, only $6 \%$ of KCs elicit an action potential even though most KCs respond to different odors with a hyperpolarization or a depolarization below firing threshold. Interestingly, no concentration dependent additional activation of KCs could be observed in contrast to OSNs and PNs. Odorant mixtures activate subsets of KCs that are not a summation of the cells activated by the single components of the mixture (similar to responses of PNs or mitral cells in the olfactory bulb of vertebrates) [Tabor et al., 2004; Deisig et al., 2006; Silbering \& Galizia, 2007; Turner et al., 2008; Honegger et al., 2011].

The division of KCs in subgroups ( $\alpha / \beta-, \alpha^{\prime} / \beta^{\prime}-$ and $\gamma$-neurons) suggested from the anatomy [Crittenden et al., 1998; Strausfeld et al., 2003; Tanaka et al., 2008] was confirmed on a functional level. Whereas $\alpha^{\prime} / \beta^{\prime}$-neurons have a broad odor tuning, the highest baseline firing rate and the strongest spiking in response to odor stimulation, $\alpha / \beta$-neurons show a decreased responsiveness and spontaneous firing rate. $\gamma$-neurons have the highest firing threshold even though subthreshold responses occur [Turner et al., 2008; Honegger et al., 2011].

Neurons that form connections within the mushroom body but also extend their arborizations to other brain regions are termed extrinsic mushroom body neurons 


\section{Introduction}

[Tanaka et al. 2008]. Hence, the projection neurons that convey the olfactory information from the antennal lobes to the MB are one subset of MB extrinsic neurons. Other prominent examples for these extrinsic neurons are aminergic neurons that innervate distinct regions in the MB [lto et al., 1998; Crittenden et al., 1998; Tanaka et al., 2008; Waddell, 2013]. Dopaminergic and octopaminergic neurons have been shown to mediate reinforcement signals to the mushroom body [Schwaerzel et al., 2003; Riemensperger et al., 2005; Selcho et al., 2009; Claridge-Chang et al., 2009; Aso et al., 2010; Waddell, 2013). The identity of neuronal subclasses responsible for aversive and appetitive memory formation have been investigated in great details in recent years [e.g. Aso et al., 2010, 2012; Liu et al., 2012] [see Waddell, 2013, for a review]. The original model of dopamine mediating aversive and octopamine appetitive reinforcement [Schwaerzel et al., 2003; Schroll et al., 2006] had to be redefined due to the identification of dopaminergic neurons that mediate appetitive memory [Liu et al., 2012]. Octopamine was proposed to be responsible for the perception of sweet sugars that were used as a positive reinforcer in reward learning and therefore act upstream of the appetitive signaling of dopaminergic neurons [Liu et al., 2012; Waddell, 2013]. Additional extrinsic mushroom body neurons have been described and sorted according to their innervation patterns by Tanaka et al. [2008]. One group of these neurons, called MB-V2, are connecting the vertical lobes of the mushroom body $\left(\alpha / \alpha^{\prime}\right)$ and the lateral horn and are implicated in memory retrieval [Séjourné et al., 2011].

Functionally, the mushroom body is the location where the association of a reinforcing stimulus (US) with a sensory stimulus (CS) is taking place [Gerber et al., 2004] as shown by an ablation of the whole mushroom body [de Belle \& Heisenberg, 1994] and mushroom body mutants [Heisenberg et al., 1985]. Additionally, a temporally restricted interruption of the mushroom body output with shibire ${ }^{\text {ts }}$ resulted in a memory impairment only during retrieval and not during acquisition which suggests the MB neurons to constitute the memory [Dubnau et al., 2001]. On a molecular level, $G$ protein signaling via the $G_{\alpha_{s}}$ subunit in the mushroom body was shown to be necessary during the association of the CS and the US [Connolly et al., 1996]. An adenylat cyclase (AC, encoded by the rutabaga-gene) was identified to act as a coincidence detector for simultaneous CS and US presentation [Zars et al., 2000; McGuire et al., 2003; Mao et al., 2004]. During associative memory formation in 


\section{Introduction}

the mushroom body, the CS is represented by an activation of a specific KC and therefore a higher $\mathrm{Ca}^{2+}$ concentration within the cell. High $\mathrm{Ca}^{2+}$-levels in turn activate the $\mathrm{Ca}^{2+}$ modulated protein calmodulin ( $\left.\mathrm{Ca}^{2+} / \mathrm{CAM}\right) . \mathrm{Ca}^{2+} / \mathrm{CAM}$ is acting as a messenger protein and influences the activity of the adenylat cyclase encoded by rutabaga. The AC is additionally regulated by the G-protein $\alpha$ - subunit that is activated upon binding of dopamine at the dopamine receptor (see Figure $1.4 \mathrm{D}$ for a scheme) [Schwaerzel et al., 2002]. The strong activity of the AC due to activation via $\mathrm{Ca}^{2+} / \mathrm{CAM}$ and the G-protein $\alpha$ - subunit results in a strongly increased cAMP level. In the absence of the US during CS activation an antagonist was proposed to be active that reduces rutabaga activity and thereby cAMP concentration during memory extinction [Schwaerzel et al., 2002; Heisenberg, 2003; Davis et al., 1995]. The importance of the cAMP pathway during memory formation is underlined by the necessity of a functional phosphodiesterase (PDE, encoded by the gene dunce) in the mushroom bodies during associative learning. PDE is acting in contrast to the AC and decreases the cAMP concentration [Dudai et al., 1976; Davis et al., 1995]. High cAMP concentrations activate protein kinase A (PKA) which is phosphorylating several downstream targets such as potassium channels and thereby directly influencing the electrical properties of the cell. Additionally, PKA activity can result in activation of further downstream signaling machanisms that influence gene expression and thereby long term memory formation, i.e. the CREB (cAMP response element-binding protein) pathway. These intracellular mechanisms are suggested to result in plastic changes that alter the responsiveness of the Kenyon cells to incoming stimuli. Thereby, the strength of the neuronal output or the number of cells responding to a stimulus is varied [Davis, 2004; McGuire et al., 2005; Tomchik \& Davis, 2009; Gervasi et al., 2010; Dubnau \& Chiang, 2013].

Apart from the molecular basis for memory formation, a lot of effort was put into the elucidation of neuronal circuits that underly the formation, consolidation and retrieval of olfactory memory. As these mechanisms are crucial to adapt an animals behavior towards similar stimuli depending on experiences, the next section will provide an overview of the experiments and the obtained results. 


\section{Introduction}

\subsection{Olfactory learning in Drosophila melanogaster}

Fruit flies can associate the presentation of an odor with a simultaneously applied reinforcement Quinn et al., 1974; Tempel et al., 1983; Heisenberg, 2003; Davis et al., 1995; Fiala, 2007; Keene \& Waddell, 2007]. Drosophila's capability to associate the presentation of an odorant with the punishing properties of an electric shock via simultaneous presentation of the two stimuli was first described by Quinn et al. [1974]. During the training phase, two different odorants were presented to the flies and one of them was temporarily paired with the application of an electric shock via a copper grid. In a subsequent test phase, the responses of the flies to both odorants was observed and an avoidance of the odorant paired with the electric shock was prominent. However, the learning observed in this paradigm is not comparable with learning as a gain of new information in a single fly. Flies that showed an avoidance of the punished odorant did not necessarily show an avoidance again if the test is repeated. Even though the number of flies avoiding the punished odorant will stay the same, a prediction for each individual can not be made. The observed learning is therefore a change in the probability to avoid the presented odorant Quinn et al., 1974; Beck et al., 2000]. The learning paradigm was further improved by changing the test situation into a T-maze test: during the test phase, both odorants are presented simultaneously in opposing tubes and the flies have to decide which side to approach (or avoid) [Tully \& Quinn, 1985].

As depicted in figure 1.6, the complete aversive associative learning protocol in flies consists of a training phase and a test phase. Groups of flies are placed in a tube in the training apparatus. A constant air stream is guided through the tube in order to transport odorant molecules along the flies. Different odorants can be placed in odorant containers (odor cups) in front of the tubes. In the training phase, two odorants (depicted in yellow and blue in figure 1.6) are presented temporally separated and one of them (e.g. yellow, the CS+) is paired with the presentation of an electric shock applied though the copper grid in the training tubes. For the test phase, the flies are transported via an elevator section to a T-maze. Each side of the T-maze contains one odorant and the flies have to decide which side to approach or avoid. Subsequently, the number of flies on each side is counted and a learning index calculated [Tully \& Quinn, 1985]. A variation of the original apparatus makes it possible 


\section{Introduction}

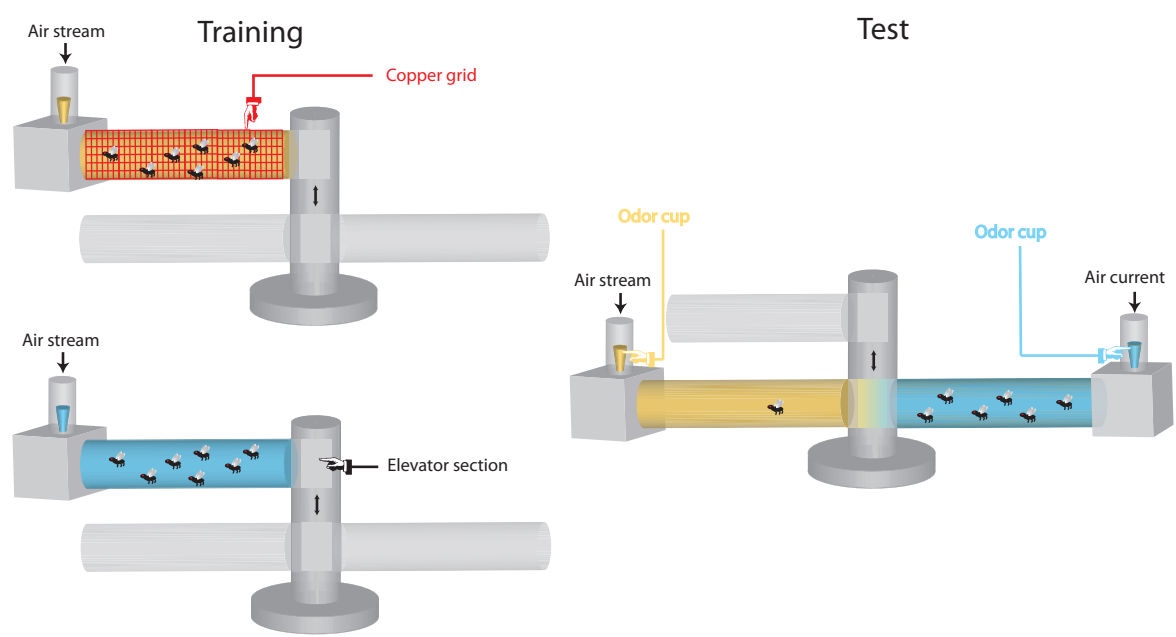

Figure 1.6. Schematic of the olfactory learning paradigm.

Groups of flies are placed in a tube with a copper grid during the training phase. Two odors ("yellow" and "blue") are presented temporally separated by placing odor containers in front of the tubes. A constant air stream ensures the transportation of the odor molecules to the flies in the tubes. During the presentation of one of the odors, the copper grid is electrified. After the training, the flies are transferred via an elevator section to a T-maze for testing. The maze consists of two tubes, each containing one of the previously presented odors. The flies have to decide which side of the T-maze to approach or avoid. The output of the test is determined by counting the number of flies on each side of the T-maze.

to train and test four groups of animals at the same time [Schwaerzel et al., 2002]. As a more naturally occurring situation than the aversive conditioning with an electric shock, appetitive olfactory learning with a sugar reward was introduced by Tempel et al. [1983]. Flies have to be starved for 18-20 hrs before they are transferred into a learning apparatus similar to the one used during aversive conditioning. Instead of an electric shock as a negative reinforcement, one of the odorants is paired with a sucrose dilution. In a subsequent T-maze test, the flies are approaching the reinforced odorant [Tempel et al., 1983].

In addition to olfactory learning, Drosophila melanogaster was also subject to other conditioning paradigms. The fruit fly was shown to be capable of visual learning, including the differentiation of distinct colors and patterns [Dill et al., 1993; Brembs \& Heisenberg, 2001]. During visual conditioning of a single fly in a flight simulator, 


\section{Introduction}

a color or a pattern is paired with a negative reinforcement. During the test, the reaction of the fly toward this pattern or color is observed. Another example for additional conditioning paradigms in adult flies is the formation of courtship memory. When a sexually naïve male fly is retracted during a courtship encounter with an already mated female, it reduces its courting efforts in the next encounter with a female [Siegel \& Hall, 1979]. Other forms of conditioning in the fruit fly that will not be further described here include proboscis extension reflex observations [Chabaud et al., 2006], spatial orientation memory [Neuser et al., 2008], phototaxic repression [Le Bourg, 2004] and several larvae learning paradigms [Aceves-Piña \& Quinn, 1979; Gerber \& Stocker, 2007] [see Pitman et al., 2009, for a review of conditioning paradigms in fruit flies].

However, olfactory learning is the most extensively studied conditioning paradigm in Drosophila. The broad term "memory" can be dissected into different forms: short term memory (STM), anesthesia sensitive memory (ASM, also called middle or intermediate term memory, MTM or ITM), anesthesia resistant memory (ARM) and long term memory (LTM) [Quinn \& Dudai, 1976; Tully et al., 1994]. Whereas STM and LTM are explained by their restricted longevity, ASM and ARM are defined by the fact that memory formation can be impaired by anesthetizing the flies on ice. Repetitions of the training procedure result in different forms of memory. Whereas a single training trial, as described above, gives rise to fast decaying STM and partially ASM, repetitions of the training (massed training) increase the time during which the memory can be recalled (ASM and ARM) [Tully et al., 1994; Margulies et al., 2005]. Repetitions of the training with pauses between the conditioning phases (spaced training) increase the longevity of the memory and a protein-synthesis-dependent LTM is formed [Tully et al., 1994; Margulies et al., 2005].

These forms of memory describe different time points during memory formation: whereas STM only addresses the acquisition and immediate recall of memory, ASM includes first phases of memory consolidation which with time converts to ARM and finally LTM. The learning scores observed during tests for associative conditioning represent an addition of the different forms of memory at the time point of the test (see figure 1.7) [Margulies et al., 2005; McGuire et al., 2005].

Several genes underlying the molecular changes in the learning process could be identified with the help of this paradigm, including the already mentioned rutabaga 


\section{Introduction}

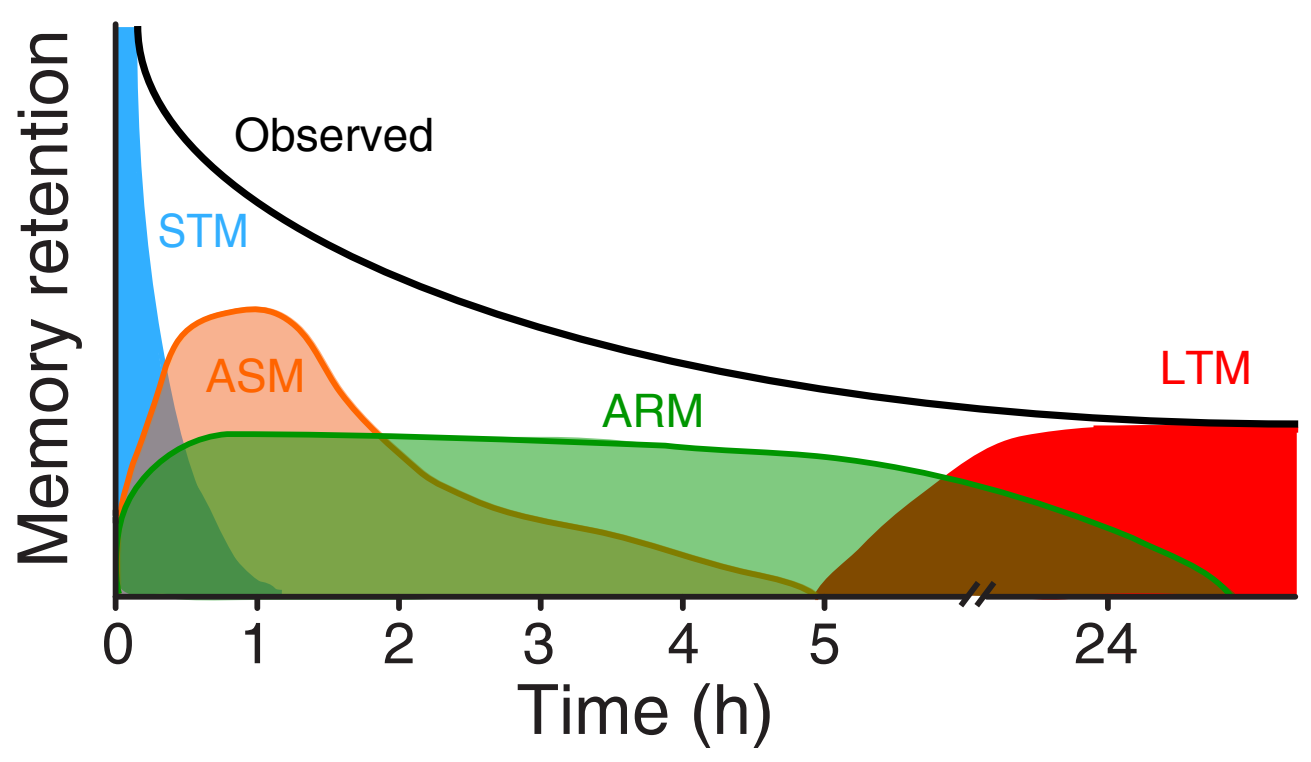

Figure 1.7. Different forms of memory.

Subdivision of the observed memory retention after olfactory conditioning. Depending on the conditioning paradigms (single training phase or repeated training trials with or without breaks between them) distinct forms of memory are formed. Short term (STM, blue), anesthesia sensitive (ASM, orange) and resistant (ARM, green) and long term memory form after varying time points and have different decay times.

Picture modified from [Margulies et al., 2005]

(rut,encoding an adenylat cyclase) [Tully \& Quinn, 1985; Zars et al., 2000] and dunce (dnc, encoding a phosphodiestrase) [Dudai et al., 1976]. Additional identified genes include neuropeptides (amnesiac [Quinn et al., 1979; [Feany \& Quinn, 1995]), PKA associated proteins (PKA-RI [Goodwin et al., 1997] and DC0 [Skoulakis et al., 1993]), various receptors (such as dopamine [Han et al., 1996; Kim et al., 2003], octopamine [Han et al., 1998] and glutamate receptor subunits (Xia et al., 2005]), transcription factors (mushroom body miniature, $\mathrm{mbm}$ [Heisenberg et al., 1985]) and proteins responsible for gene expression (e.g. dCREB-2 [Yin et al., 1994] or the translation initiation factor elF-5C [Dubnau et al., 2003]) [see Keene \& Waddell, 2007, for a review]. Mutations of these learning related genes provided a powerful tool to investigate neuronal circuits and brain regions involved in the formation, consolidation and retrieval of olfactory memory. 


\section{Introduction}

\subsubsection{Neuronal plasticity in the antennal lobe}

The neuronal response to an odor stimulation in form of activity patterns can be observed with $\mathrm{Ca}^{2+}$ imaging [Fiala et al., 2002]. Even though the mushroom body is necessary for the association of an olfactory stimulus (CS) with a reinforcement (US) and an ablation of the mushroom body resulted in a severe learning deficit, plastic changes could also be observed in the first olfactory neuropil, the antennal lobe [Faber et al., 1999; Yu et al., 2004; Peele et al., 2006; Sachse et al., 2007; Das et al., 2011; Sudhakaran et al., 2012]. Changes in the neuronal responses to odorant presentation could be found, for example, as a result of olfactory habituation [Das et al. 2011; Sudhakaran et al., 2012]. An exposure of flies with an odorant before the imaging procedure resulted in a decreased calcium signal reflecting a weaker stimulation of the projection neurons when compared to flies that were not exposed. These changes are mediated by a feedback from the PNs to GABAergic inhibitory local interneurons type I (LN1) during the habituation phase. Additionally, prolonged exposure to an increased $\mathrm{CO}_{2}$-concentration was shown to lead to a larger volume of the responding V-glomerulus and a decrease in the neuronal response to $\mathrm{CO}_{2}$ in projection neurons [Sachse et al., 2007].

Whether plasticity in the antennal lobe is also happening in response to olfactory conditioning remains unclear. Imaging studies in the honey bee antennal lobe [Faber et al., 1999] and projection neurons in the fruit fly [Yu et al., 2004] suggest an alteration of the response to the odor used as $\mathrm{CS}_{+}$. Whereas an increased signal could be observed in the honey bee, additional projection neurons were recruited in Drosophila. In both studies, the responses to the CS- did not change. In contradiction to these results, Peele et al. [2006] did not observe any learning induced changes in the calcium activity in the antennal lobe after olfactory conditioning.

Despite the changes due to habituation and possible changes due to olfactory conditioning in the antennal lobe, the functional role of the mushroom body in learning processes is very well described. An extensive amount of research has been done in the recent years and the next paragraph will provide an overview about plastic changes in the mushroom body intrinsic and extrinsic neurons in response to olfactory learning. 


\section{Introduction}

\subsubsection{Learning induced plasticity in the mushroom body}

The function of the mushroom body during olfactory learning was first implicated in the honey bee after the specific disruption of neuronal function in the calyx or the $\alpha$-lobes with a cooled metal after learning [Erber et al., 1980]. More evidence could be found in the ablation or mutation of the MB which resulted in a phenotype of deficient learning [Heisenberg et al., 1985; de Belle \& Heisenberg, 1994; Gerber et al., 2004]. Additionally, it could be shown that the reinforcing signal of punishment and reward is mediated by dopaminergic neurons that innervate different regions of the mushroom body [Schwaerzel et al., 2003; Riemensperger et al., 2005; Selcho et al., 2009; Claridge-Chang et al., 2009; Aso et al., 2010; Liu et al., 2012]. However, dopaminergic neurons conveying different reinforcement signals are innervating distinct regions in the mushroom body [Liu et al., 2012; Aso et al., 2012; Waddell, 2013]. The Kenyon cells are proposed to act as coincidence detectors of the conditioned stimulus (CS, the presentation of an odor) and an unconditioned stimulus (US, the electric shock in aversive and the sugar reward in appetitive learning) [Zars et al., 2000; McGuire et al., 2003; Mao et al., 2004; Szyszka et al.]. As described above, for this coincidence detection (and therefore for the acquisition of memory) a functional adenylat cyclase rutabaga in subsets of Kenyon cells is necessary [Zars et al., 2000; McGuire et al., 2003; Mao et al., 2004; Blum \& Dubnau, 2010]. On the contrary, it was originally unclear if the output from the mushroom body intrinsic neurons is needed during the acquisition of memory. However, the retrieval of the formed associations is only possible with intact synaptic connections between the Kenyon cells and mushroom body output neurons [Dubnau et al., 2001; Schwaerzel et al., 2002; McGuire et al., 2003; Heisenberg, 2003]. This primary result was challenged by the possibility to specifically target subclasses of Kenyon cells with different GAL4-driver lines [Yao Yang et al., 1995]. Additionally, the distinct forms of memory could be assigned to different subclasses of intrinsic mushroom body neurons depending on the time course of genetic manipulation of the circuit [Zars et al., 2000; Pascual, 2001; Krashes et al., 2007; Blum \& Dubnau, 2010].

In order to form an aversive olfactory memory, the odorant (CS) and the punishing electric shock as a reinforcer (US) have to perceived in a temporally overlapping time [Zars, 2010]. As the reinforcement is mediated via dopaminergic neurons and the odorant is activating Kenyon cells in the mushroom body, the neurons that detect 


\section{Introduction}

the temporal coincidence of the two stimuli have to respond to dopamine release. A disruption of the expression of dopamine receptors is therefore preventing the acquisition of olfactory memory [Kim et al., 2007]. A normal expression of the dopamine receptor DopR only in the $\gamma$-neurons of the mushroom body is sufficient to rescue this memory impairment (in all forms of memory), suggesting a dopaminergic input in the $\gamma$-lobe as a basis for memory acquisition [Qin et al., 2012].

Output from $\alpha^{\prime} / \beta$ '-neurons on the other hand has been shown to be required during memory acquisition and consolidation [Krashes et al., 2007]. This finding could be supported by functional imaging. A memory trace, i.e. a stronger response towards a previously punished odorant, could be found in $\alpha^{\prime} / \beta^{\prime}$-neurons up to one hour after the training [Wang et al., 2008]. In addition to the $\alpha^{\prime} / \beta^{\prime}$-neurons, APL and DPM neurons were found to be required during memory consolidation. A memory trace could be found in DPM neurons 30 minutes after training and output from the DPM neurons to the $\alpha^{\prime} / \beta^{\prime}$-Kenyon cells is necessary during the consolidation process $\mid \mathrm{Yu}$ et al., 2005; Keene et al., 2006; Cervantes-Sandoval \& Davis, 2012]. GABAergic APL neurons also show a memory trace but the measured calcium response is decreased and not increased directly after the learning phase. Additionally, a decrease of GABA release from APL neurons increases learning. The activity of APL neurons is thereby suppressed and also suppresses memory formation [Liu \& Davis, 2009]. Interestingly, APL and DPM are electrically coupled and the electric communication is required for memory consolidation of anesthesia sensitive memory. APL and DPM neurons therefore play a key role in the recurrent memory consolidation loop that interconnect the $\alpha^{\prime} / \beta^{\prime}$ and $\alpha / \beta$-lobes. Whereas DPM is suggested to facilitate the changes in neurons activated by the CS+, APL is sharpening the response by inhibiting activity in the cells that are not associated with the reinforcement [Wu et al., 2011; Pitman et al., 2011; Dubnau \& Chiang, 2013].

Whereas the early forms of consolidation lead to STM, ASM and partially ARM, a longer consolidation triggered by repeated spaced training is necessary to form LTM. A mutational ablation of the vertical lobes of the mushroom body $\left(\alpha / \alpha^{\prime}\right)$ resulted in flies that are fully capable of STM but fail to form LTM after spaced training [Pascual, 2001]. A correlate of LTM in the vertical lobes could also be confirmed with functional imaging. A memory trace in the $\alpha$-lobe could be found $9 \mathrm{hrs}$ after training whereas no change was observed in the same lobe $3 \mathrm{hrs}$ after training. This memory trace 


\section{Introduction}

is not present when the CREB-pathway is interrupted and thereby LTM formation prevented or when the flies are trained in a training paradigm that does not result in LTM [Yu et al., 2006; Akalal et al., 2010]. The memory trace can still be observed $24 \mathrm{hrs}$ after training but decays after $48 \mathrm{hrs}$. Interestingly, the memory trace could not be found in the $\beta$-branch of the same neurons which suggest a branch specific plasticity [Yu et al., 2006; Akalal et al., 2010; Davis, 2011]. Interestingly, the investigation of several LTM learning mutants showed an absence of the LTM memory trace in the $\alpha$-lobes in all mutants, independent of the individual proteins targeted by the mutation [Akalal et al., 2011]. A memory trace after olfactory conditioning in extrinsic mushroom body neurons in response to the CS+ could also be found in honey bees. Additionally, the increase in the calcium signal was not only observed for the CS+ but also in response to a similar odorant in contrast to a dissimilar odorant [Haehnel \& Menzel, 2012].

In addition to the memory traces that were found during the consolidation phase in mushroom body intrinsic neurons, a memory trace was also found in a pair of extrinsic MB neurons [Séjourné et al., 2011]. MB-V2 extrinsic neurons connect the mushroom body $\alpha$ and $\alpha$ '-lobes and the lateral horn and respond to odor application with an increase in the calcium signal. After aversive conditioning, the MB-V2 neurons show a decreased calcium signal in response to the conditioned odorant. The synaptic transmission of these neurons is essential during memory retrieval after aversive conditioning. Therefore, they are implicated in conveying the information for avoidance behavior towards the punished odorant to the lateral horn [Séjourné et al., 2011]. Recent studies could also identify additional neurons involved in the storage of LTM: the dorsal anterior lateral (DAL) neurons [Chen et al., 2012]. Visualization of de-novo protein synthesis showed a strong signal during LTM consolidation in the DAL neurons whereas the MB neurons were not detectable. Additionally, a disruption of protein synthesis specifically in the DAL neurons impairs LTM. However, DAL output is only necessary during memory retrieval and not memory acquisition or consolidation. It was therefore proposed that the information is conveyed from the mushroom body to the DAL neurons for memory storage and is later retrieved by the MB. This feedback loop for memory storage and retrieval is supported by a functional connection between the DAL neurons and $\alpha / \beta$ mushroom body neurons in the calyx [Chen et al., 2012; Dubnau \& Chiang, 2013]. 


\section{Introduction}

Interestingly, it could also be shown that appetitive STM and LTM are formed independently in different subsets of mushroom body neurons [Trannoy et al., 2011]. Whereas appetitive STM is strongly relying on activity and rutabaga expression in $\gamma$-neurons, $\alpha / \beta$-neurons are necessary for appetitive LTM. Likewise, output from $\gamma$-neurons is important during STM retrieval but irrelevant in LTM and output from $\alpha / \beta$-neurons is only required for LTM retrieval [Trannoy et al., 2011]. A similar result was obtained with aversive conditioning and a disruption of cAMP signaling in subsets of KCs during STM, ASM and ARM [Scheunemann et al., 2012].

Conclusively, the mushroom body plays a crucial role in memory acquisition, consolidation and retrieval. The substructures of the mushroom body have distinct functions during the different phases. Whereas dopaminergic signaling to the $\gamma$-neurons are required for memory acquisition of STM, acquisition and consolidation is governed by $\alpha^{\prime} / \beta$ '-neurons and the KC-DPM/APL-KC feedback loop during ASM and ARM. The information is further conveyed during LTM consolidation to the DAL neurons which in

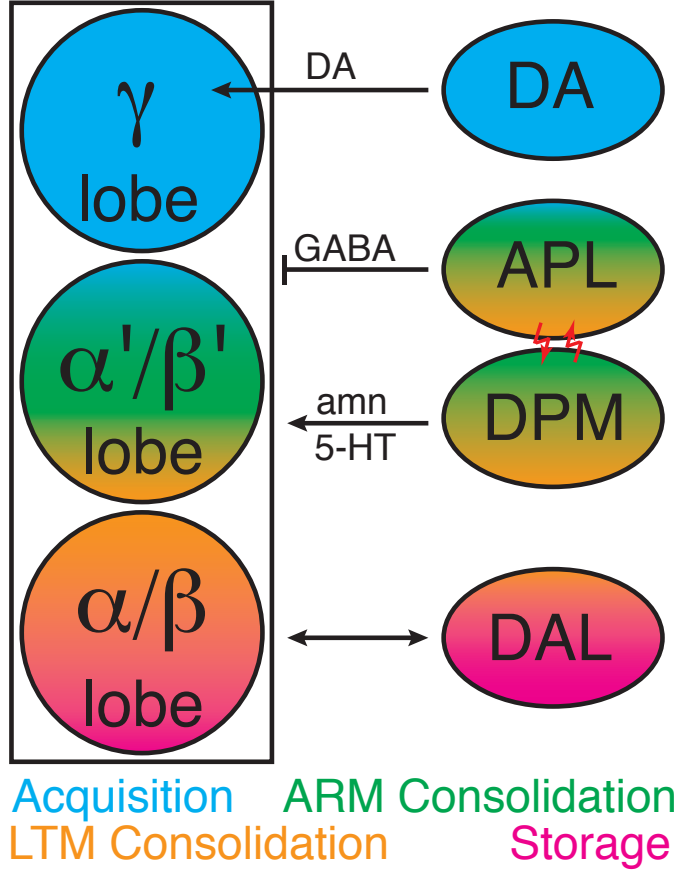

Figure 1.8. Scheme of the intrinsic and extrinsic mushroom body neurons during the different phases of learning. Dopaminergic signaling to the $\gamma$-neurons is crucial during memory acquisition. During acquisition and memory consolidation, the $\alpha^{\prime} / \beta^{\prime}$-neurons and the MB-DPM/APL-MB feedback loop with electrical synapses between APL and DPM neurons are necessary. Whereas APL releases inhibitory GABA on the Kenyon cells, DPM is releasing serotonin (5-HT) and the amnesiac gene product. Further consolidation, storage and retrieval of long-term memory requires the interaction between $\alpha / \beta-K C$ s and DALneurons.

turn feed back to $\alpha / \beta$-neurons (MB-DAL-MB feedback loop) (see Figure 1.8). During retrieval, the information is transferred via the MB-V2 neurons to the lateral horn. The described pathway of olfactory information processing, including the locations for possible plasticity, is the foundation for behavioral responses towards odors. The generalization of similar odorants is mediated by these networks. A change of the 


\section{Introduction}

importance of a specific odorant by the association of the odorant with a reinforcement results in an alteration of the behavioral response. Underlying this alteration are changes in the neuronal circuitry along the olfactory pathway. Hence, a learned discrimination of similar odorants, and thereby a change in the perception of the odorants, probably has a neuronal correlate within the described structures.

\subsection{Aim of the study}

The ability of an animal to react with an appropriate response to a volatile chemical stimulus (odor) is of crucial importance. Depending on prior experiences, the animal has to be able to evaluate an incoming smell and decide how to react to the stimulus. As stimuli in nature are rarely exactly overlapping, slight differences in odor composition and thereby similar chemical information have to be generalized in most situations. If an odor is associated with a (positive or negative) reinforcement, a second odor that is similar to the conditioned odor and does not have any prior value should therefore be generalized. However, in some cases a fine discrimination of similar odors is beneficial. When an odor is associated with a reinforcement and a similar odor is explicitly not reinforced, the generalization of the similar odors should decrease and the difference between the odors detected. The olfactory system of an animal should therefore be able to adept its acuity, and thereby the perception of an odorant, depending on the situation. A change of the perception of odors can not be addressed directly as a measure for perception is unknown. This problem is addressed during this study by investigating two aspects of odor perception: behavioral output and physiological responses of neurons to odor stimulation. These complementary approaches are providing an indirect access to explain perceptual changes due to experiences.

Most olfactory learning experiments in Drosophila have been performed with a specific set of odorants that were introduced in the early years of olfactory conditioning in the fruit fly: benzaldehyde, 4-methylcyclohexanol and 3-octanol [Quinn et al., 1974; Dudai et al., 1976]. These three odorants are chemically very distinct and therefore result in strong robust learning scores. Nevertheless, the choice of odors is of great importance during olfactory learning experiments. During the investigation of genes or circuits that might be necessary or sufficient for the learning process, the choice 


\section{Introduction}

to use very distinct odors is comprehensible. However, in order to investigate perceptual changes towards odors depending on the situation, these odorants are not suitable. As they are already discriminable before conditioning, it is not possible to investigate an increase in discrimination in response to a conditioning experience. Therefore, two structurally similar odorants that were shown to be generalized after an absolute conditioning paradigm by Drosophila larvae were used during this study: 1-octen-3-ol (1-Oct) and 3-octanol (3-Oct) [Mishra et al., 2010]. As a dissimilar control odor, 4-methylcyclohexanol (MCH) was used (Figure 1.9). The similar odorants both consists of eight carbon atoms in a chain with an alcohol group connected to the third carbon. The only difference is a double binding between the first two carbon atoms in 1-Oct. $\mathrm{MCH}$ on the other hand has a very different chemical structure. Six of its carbon atoms are forming a circle and the alcohol group is connected to the opposite side of a methyl group.

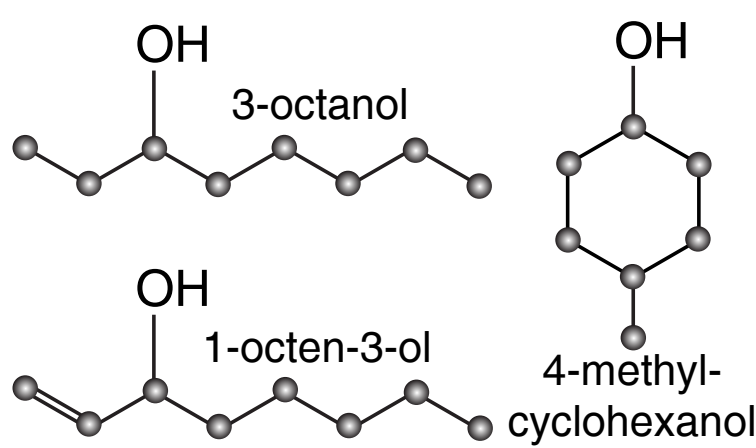

Figure 1.9. Chemical structures of
1-Oct, 3-Oct and MCH.
1-octen-3-ol and 3-octanol have very
similar chemical structures consisting
of a chain with 8 carbon atoms with
the alcohol group at the third one. The
only difference is a double binding be-
tween the first two carbon atoms of the
chain. 4-methylcyclohexanol has a very
distinct chemical structure with a ring
formed by six carbon atoms.

The two similar odorants are providing an opportunity to investigate possible changes in the perception of odors in response to different training paradigms. Is it possible to alter the olfactory acuity of the fruit fly? This study is investigating the ability of Drosophila to change the olfactory acuity and thereby the perception of an odor depending on the situation. First, it was investigated on a behavioral level whether two distinct odorants can be made more similar by a combined presentation (sensory preconditioning). Secondly, two similar odorants were used to investigate the responses of flies to different training paradigms. Can Drosophila learn to discriminate two similar odorants that were generalized after absolute conditioning? And if so, which neuronal circuits along the olfactory pathway are necessary to show 


\section{Introduction}

such an enhanced olfactory acuity? In a third step, the differential learning paradigm was dissected into its two parts, conditioned excitation and conditioned inhibition, in order to find the mechanisms responsible for a decreased generalization after differential conditioning. Fourth, it was investigated if an enhancement of olfactory acuity is dependent on the olfactory input during the retrieval of the memory and if the molecular identity of the two similar odorants is important during the discrimination. Finally, it is of interest to localize a possible neural correlate for the learned discrimination after differential conditioning in contrast to generalization after absolute conditioning within the olfactory pathway. 


\section{Materials and Methods}

\subsection{Materials}

\subsubsection{Fruit flies and bacteria}

\section{Escherichia coli}

During the generation of new transgenic flies, three different strains of Escherichia coli bacteria were used: OneShot ${ }^{\circledR}$ Mach1 competent cells (Life technologies; Carlsbad, CA, USA) from the Gateway ${ }^{\circledR}$ cloning system [Hartley et al., 2000] were available during Gateway ${ }^{\circledR}$ cloning. DB3.1 cells (Life technologies; Carlsbad, CA, USA) were necessary to amplify the LexA backbone vectors (see section 2.1.2) for the Gateway ${ }^{\circledR}$ cloning as the plasmids contains a $c c d B$ sequence which induces cell death (DB3.1 cells contain the gyrA462 allele which confers resistance to $c c d B$ ). Plasmids were amplified and screened in XL1-Blue competent cells (Promega; Fitchburg, WI, USA).

\section{Drosophila melanogaster strains}

Wild type flies of the Canton-S strain have been used for the behavioral experiments, if not stated otherwise.

In order to specifically target local interneurons in the antennal lobe, the GAL4enhancer-trap lines NP1227 $\left(w^{-}, y ; \frac{N P 1227}{C y O} ;+\right)$ and NP2426 $(N P 2426 ;+;+)$ have been used. NP1227-GAL4 and NP2426-GAL4 express in different subsets of local interneurons and have distinct functions and properties [Sachse et al., 2007; Okada et al., 2009; Seki et al., 2010].

For visualization of neurons targeted by the GAL4 lines (see section 3.7.1), a fly strain expressing GFP was the transgene of choice. In order to visualize the whole 


\section{Materials and Methods}

neuronal structure, GFP expression is targeted to cellular membranes and synapses due to a coupling of the GFP with mCD8 and synaptobrevin, respectively. These flies $\left(w^{-} ; U A S-m C D 8: G F P, U A S-s y b: G F P ;+\right)$ were kindly provided by Dr. Serge Birman.

For blocking synaptic transmission of neurons with the targeted expression of shibire as described in section 1.3.2, a fly strain with two copies of the temperature sensitive shibire $^{t s}$ allele on the X-chromosome and the third chromosome under the control of UAS $\left(w^{-}, U A S-s h i^{t s} ;+; U A S-s h i^{t s}\right)$ was chosen [Kitamoto, 2001].

As a genetic control of the heterozygous combinations of shibire ${ }^{t s}$ and NP2426, the homozygous parental lines were combined with flies of $\mathrm{W}^{1118}$ strain to work with heterozygous genetic controls as well (see section 3.7.2).

The calcium sensor GCaMP3.0 is a well established tool to monitor neuronal activity (see section 1.3.3). Therefore, flies with an expression of GCaMP3.0 under the control of UAS [Tian et al., 2009] were used to express GCaMP specifically in sensory neurons. To image neuronal activity specifically in olfactory sensory neurons, an Or83b-GAL4 driver line [Wang et al., 2003a; Larsson et al., 2004] was chosen. A fly strain with homozygous expression of Or83b-GAL4 and UAS-GCaMP3.0 was available in the laboratory (+;UAS - GCaMP3.0;Or $83 b-G A L 4)$. The neuronal activity in the mushroom body was monitored with a new fly strain created by Ulrike Pech. The flies express GCaMP3.0 directly under the control of the mb247promotor which specifically targets Kenyon cells [Schulz et al., 1996; Zars et al., 2000; Riemensperger et al., 2005].

Expression of GCaMP3.0 of the newly generated LexOp:GCaMP3.0 fly line was confirmed with a fly line expressing LexA:VP16 under the control of the Or83b-promotor (Or83b-LexA:VP16 [Lai \& Lee, 2006]). These flies were kindly provided by Dr. Silke Sachse (Max Planck Institute for Chemical Ecology, Jena, Germany) and also by Prof. Hitoshi Okazawa (University of Tokyo, School of Medicine, Tokyo, Japan).

\subsubsection{Constructs and plasmids}

Different bacterial vectors have been used to generate new transgenic fly strains. With the help of the Gateway ${ }^{\circledR}$ cloning system [Hartley et al., 2000] a fly strain with the expression of the Calcium sensor GCaMP3.0 [Tian et al., 2009] under the control 


\section{Materials and Methods}

of the lexA system [Lai \& Lee, 2006] was generated. Sören Diegelmann provided us with a backbone vector for the LexA-system (pLOT-W) to integrate target DNA in a Drosophila injection vector for the Gateway ${ }^{\circledR}$ system [Diegelmann et al., 2008]. In addition to these destination vectors, the $\mathrm{pCR}{ }^{\circledR} 8 / \mathrm{GW} / \mathrm{TOPO}{ }^{\circledR}$ vector from invitrogen (Life technologies; Carlsbad, CA, USA) was chosen as entry vector.

The backbone vector pCaSpEr:mb247 ([Riemensperger et al., 2005]) was chosen to target the expression of a red calcium sensor (R-GECO1.0 [Zhao et al., 2011]) to mb247 positive cells in the mushroom body.

To generate flies with a specific expression of a transgene under UAS control, the pUASt vector was used as a backbone for fly injections [Brand \& Perrimon, 1993].

The DNA sequence of the red calcium sensor R-GECO1.0 was obtained from AddGene (Cambridge, MA, USA).

\subsubsection{Media}

Fly food For 10 I of food medium, $102.5 \mathrm{~g}$ Agar (Probio; Rödermark, Germany) were dissolved in $5 \mathrm{I}$ of water by boiling at $99.5^{\circ} \mathrm{C}$ for two hours. Subsequently, the following ingredients were added:

$\begin{array}{llll}100 & \mathrm{~g} & \text { Soy flour } & \\ 180 & \mathrm{~g} & \text { Yeast } & \text { dissolved in 1I water } \\ 800 & \mathrm{~g} & \text { Corn flour } & \text { dissolved in 2l water } \\ 220 & \mathrm{~g} & \text { Treacle } & \text { dissolved in 1I water } \\ 800 & \mathrm{~g} & \text { Malt } & \text { dissolved in 1I water }\end{array}$

The solution was left for cooling until $55-60^{\circ} \mathrm{C}$ was reached. Then,

$63 \mathrm{ml}$ Propionic acid (Carl Roth GmbH + Co KG; Karlsruhe, Germany)

15 g Nipagin (Sigma-Aldrich; St. Louis, MO, USA) dissolved in

$50 \mathrm{ml}$ pure ethanol (VWR International GmbH; Darmstadt, Germany) were added and the food was filled into plastic vials (Sarstedt; Nümbrecht, Germany) and stored at $4{ }^{\circ} \mathrm{C}$ until usage.

Standart LB medium (Lysogeny broth medium): bacteria were inoculated in standart LB medium for vector amplification: 


\section{Materials and Methods}

To get one liter of LB media

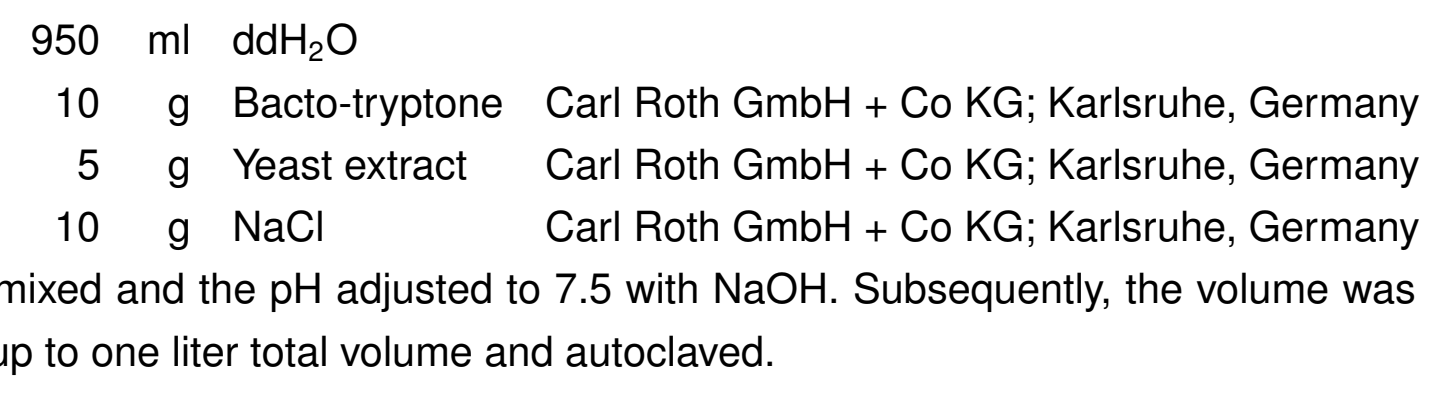

LB-Agar: clones were grown on LB-Agar and selected depending on their antibiotic resistance.

1 | LB medium

15 g Agar Carl Roth GmbH + Co KG; Karlsruhe, Germany

was mixed and autoclaved. After a short cooling period, $1 \mathrm{ml}$ of the appropriate antibiotic was added to the liquid medium before it is poured onto petridishes to solidify and stored at $4{ }^{\circ} \mathrm{C}$ until usage.

SOC medium (Super Optimal broth with Catabolite repression) was delivered with the kit from invitrogen (life technologies; Carlsbad, CA, USA) and used during bacterial transformation. It consists of double distilled water $\left(\mathrm{ddH}_{2} \mathrm{O}\right)$ and

$\begin{array}{rll}2 & \% & \text { Bacto-tryptone } \\ 0.5 & \% & \text { Yeast extract } \\ 10 & \mathrm{mM} \mathrm{NaCl} \\ 2.5 & \mathrm{mM} \mathrm{KCl}\end{array}$

\subsubsection{Reagents and consumables}

Antibiotics Ampicillin and chloramphenicol were bought from Carl Roth $\mathrm{GmbH}+$ Co KG (Karlsruhe, Germany) and spectinomycin from Sigma-Aldrich (St. Louis, MO, USA). Each of the antibiotics was stored in a $100 \mathrm{mg} / \mathrm{ml}$ stock solution and used in a final concentration of $100 \mu \mathrm{g} / \mathrm{ml}$. 


\section{Chemicals}

Ethidium bromide

Carl Roth GmbH + Co KG; Karlsruhe, Germany

SDS (sodium dodecyl sulfate) Carl Roth GmbH + Co KG; Karlsruhe, Germany

Agarose Carl Roth GmbH + Co KG; Karlsruhe, Germany

\section{Consumables}

Parafilm

Pechiney Plastic Packaging; Chicago, IL, USA

Minutien needles

Fine Science Tools GmbH; Heidelberg, Germany

Forceps

Fine Science Tools GmbH; Heidelberg, Germany

Pipette tips

Sarstedt; Nürmbrecht, Germany

Eppendorf tubes

Sarstedt; Nürmbrecht, Germany

\subsubsection{Odorants}

All odorants and the diluent, mineral oil, were purchased from Sigma-Aldrich (St. Louis, MO, USA) with the following CAS numbers:

$\begin{array}{lll}\text { Mineral oil } & \text { OIL } & \text { CAS 8042-47-5 } \\ \text { 3-Octanol } & \text { 1-Oct } & \text { CAS 589-98-0 } \\ \text { 1-Octen-3-ol } & \text { 3-Oct } & \text { CAS 3391-86-4 } \\ \text { 4-Methylcyclohexanol } & \text { MCH } & \text { CAS 589-91-3 } \\ \text { Benzaldehyde } & \text { BA } & \text { CAS 100-52-7 }\end{array}$

\subsubsection{Buffers and solutions}

All reagents used to prepare buffers and solutions were ordered from Carl Roth $\mathrm{GmbH}=\mathrm{Co} \mathrm{KG}$ (Karlsruhe, Germany) unless stated otherwise. 


\section{Materials and Methods}

PBS (phosphate buffered saline)

$15 \mathrm{mM} \mathrm{NaH}_{2} \mathrm{PO}_{4}$

$100 \mathrm{mM} \mathrm{NaCl} \quad$ AppliChem; Darmstadt, Germany

$85 \mathrm{mM} \mathrm{Na}_{2} \mathrm{HPO}_{4}$

dissolved in $\operatorname{dd} 2 \mathrm{H}_{2} \mathrm{O}$. The $\mathrm{pH}$ was adjusted to 7.4 with $\mathrm{HCl} / \mathrm{NaOH}$.

TBE (Tris-borate-EDTA) buffer was used for gel electrophoresis.

1 M Tris

$100 \mathrm{mM}$ EDTA

$833.4 \mathrm{mM}$ borate

dissolved in $\operatorname{dd} 2 \mathrm{H}_{2} \mathrm{O}$. The $\mathrm{pH}$ was adjusted to 8.0 with $\mathrm{HCl} / \mathrm{NaOH}$.

Ringer's solution Fly Ringer solution was necessary to keep the dissected tissue alive during preparation of fly brains for immunostainings and during the preparation of the fly for two-photon imaging:

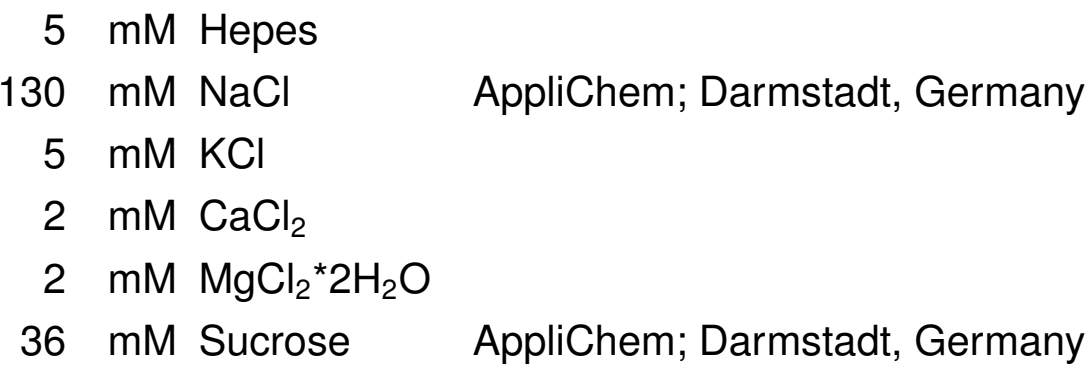

adjust $\mathrm{pH}$ to 7.3 with $\mathrm{NaOH}$ and stored until usage at $-20^{\circ} \mathrm{C}$.

PFA (4\%) (Paraformaldehyde): dissected fly brains were fixed in 4\% PFA. $25 \mathrm{ml}$ PBS

1 g Paraformaldehyde

$2.5 \mu \mathrm{l} \quad \mathrm{NaOH}$ to achieve a $\mathrm{pH}$ of 7.4

The PBS has to be heated to $60^{\circ} \mathrm{C}$ in order to dissolve the paraformaldehyde.

PBST increased the permeability of the cell membranes for the antibodies after fixation during immunostainings.

$$
\begin{array}{lll}
100 & \mathrm{ml} & \text { PBS } \\
0.6 & \mathrm{ml} & \text { Triton-X100 }
\end{array}
$$

PBST was prepared fresh and stored for maximum 2 weeks at $5{ }^{\circ} \mathrm{C}$. 


\section{Materials and Methods}

PAT was used during immunostainings to block unspecific binding of antibodies.

$$
\begin{array}{rrl}
100 & \mathrm{ml} & \text { PBST } \\
2 & \mathrm{~g} & \text { bovine serum albumin }
\end{array}
$$

PAT was prepared fresh and stored for maximum 2 weeks at $5{ }^{\circ} \mathrm{C}$.

\subsubsection{Enzymes}

In order to generate new transgenic fly strains, the following DNA modifying enzymes have been taken together with the buffer delivered by the company. The enzymes were purchased from Fermentas (Thermo scientific; Waltham, MA, USA) unless stated otherwise:

Ncol

Nhel

Sacl

BamHI

EcoRI

Notl

KspAI

Bglll

T4 Ligase

DreamTAG polymerase

Phusion polymerase FINNZYMES (Thermo scientific; Waltham, MA, USA)

dNTPs

LR Clonase ${ }^{\circledR}$ II

Reaction Mix Invitrogen (life technologies; Carlsbad, CA, USA)

$\mathrm{TOPO}^{\circledR}$ cloning kit Invitrogen (life technologies; Carlsbad, CA, USA) 


\section{Materials and Methods}

\subsubsection{Antibodies}

The bruchpilot antibody ( $\alpha$-nc82, raised in mouse [Hofbauer, 1991; Wagh et al., 2006]) was used to label the neuropil of the fly brain in order to get an orientation of the labeled structures. As secondary antibody against mouse, $\alpha$-mouse-Cy3 raised in goat from Jackson ImmunoResearch Laboratories, Inc. (West Grove, PA, USA) was chosen.

In order to increase the signal of neurons that are labeled via GFP-expression, $\alpha$-GFP raised in rabbit was used in combination with $\alpha$-rabbit-Alexa488 (both from Invitrogen, Life technologies; Carlsbad, CA, USA). Despite the above mentioned antibodies, Vectashield ${ }^{\mathrm{TM}}$ mounting medium for fluorescence was bought from Vector Laboratories, Inc. (Burlingame, CA) to stabilize the fly brains until the pictures were taken.

\subsubsection{Laboratory equipment}

\section{General equipment}

Stereo microscope (Stemi2000) Carl Zeiss AG; Oberkochen, Germany

Water bath

Memmert GmbH + Co. KG;

Schwabach, Germany

Thermomixer compact

Eppendorf; Hamburg, Germany

Incubator Certomat ${ }^{\circledR}$ BS-1

Sartorius Stedim biotech;

Göttingen, Germany

IKA ${ }^{\circledR}$ Vortex Genius 3

IKA ${ }^{\circledR}$-Werke GmbH \& CO. KG;

Staufen, Germany

Table centrifuges 5418 \& 5804R Eppendorf; Hamburg, Germany

ELISA reader EPOCH

BioTek Instruments $\mathrm{GmbH}$;

Bad Friedrichshall, Germany

Gel chamber

PEQLAB Biotechnologie GMBH;

Erlangen, Germany

UV-transilluminator BioView

biostep $\mathrm{GmbH}$; Jahnsdorf, Germany

Genoplex for gel documentation

VWR International; Radnor, PA, USA

Sorvall RC6+ Cetrifuge

Thermo scientific; Waltham, MA, USA

Pipettes

Eppendorf; Hamburg, Germany 


\section{Materials and Methods}

Setup for olfactory learning All behavioral experiments have been performed under constant temperature and humidity conditions. Therefore, a box was constructed by the university workshop and all experiments were done inside this box. The humidity was maintained at $60-80 \%$ with the air humidifier W3100E from Wick (Proctor\&Gamble; Cincinnati, OH, USA). The temperature was controlled with the electric CLAtronic heater from CTC Clatronic International GmbH (Kempen, Germany).

For the olfactory learning experiments, an apparatus named Tully-machine was used to perform four experiments in parallel (see section 1.5 and [Schwaerzel et al., 2002]). The machine was custom built by Con-Elektronik (Greussenheim, Germany). To ensure a constant timing of the electric shocks, the original machine was completed with an universal-pulse-generator UPG100 from ELV Elektronik AG (Leer, Germany). The pulse generator was programmed to trigger 12 electric shocks, each lasting $1.25 \mathrm{sec}$, with a constant break of $3.75 \mathrm{sec}$ between the pulses.

Imaging setup The fly preparations for the in-vivo calcium imaging measurements were performed at a SZX12 microscope from Olympus (Tokyo, Japan). The flies were prepared in a self-made fly holder which made it possible to punish the fly with an electric shock under the microscope. The fly holder was created with a microscope slide (VWR International GmbH; Darmstadt, Germany), a fine stainless steel metal mesh (diameter of wire $\sim 0.3 \mathrm{~mm}$ ) and some copper wire. Additionally, the following materials were used during the preparation:

Adhesive tape

$0.1 \mathrm{~mm}$ razor blades

Blade holder

Forceps

Syringe "Inject ${ }^{\circledR} "$

$0.5 \times 25 \mathrm{~mm}$ syringe needle Low melting Agarose tesa SE; Hamburg, Germany

Martor KG; Solingen, Germany

Fine Science Tools GmbH; Heidelberg, Germany

Fine Science Tools GmbH; Heidelberg, Germany

B. Braun Melsungen AG; Melsungen, Germany

Terumo Corporation; Tokyo, Japan

Sigma-Aldrich; St. Louis, MO, USA 


\section{Materials and Methods}

The calcium imaging was performed using an LSM 7 MP 2-photon microscope from Zeiss (Carl Zeiss AG; Oberkochen, Germany). The microscope was equipped with a mode-locked Ti-sapphire Chameleon Vision II laser (Coherent; Santa Clara, CA, USA) tuned to 680-1080 nm, Zeiss band-pass filters for GCaMP3.0, a Zeiss CCD camera AxioCam MRm and a Plan-Apochromat 20x/1.0 NA water immersion objective (Carl Zeiss AG; Oberkochen, Germany). Image acquisition was controlled with the ZEN black software (Carl Zeiss AG; Oberkochen, Germany). Attached to the microscope was a device, called olfactometer, to deliver odorants with a constant flow rate of $1 \mathrm{ml} / \mathrm{sec}$ directly to the fly (for a detailed description, see supplemental experimental procedures of [Riemensperger et al., 2005] and [Spall, 2005]). The onset of the odor stimulus as well as the duration could be regulated via a custom made program written with LABVIEW (National instruments; Austin, TE, USA). The olfactometer was custom-built by Con-Elektronik (Greussenheim, Germany).

\subsection{Methods}

\subsubsection{Fly husbandry}

Flies were raised on standard fly food (see section 2.1.3) in a climate chamber at $25^{\circ} \mathrm{C}, 60 \%$ humidity and a light/dark cycle of $12 \mathrm{~h} / 12 \mathrm{~h}$. The flies raised for behavioral experiments were kept in mass culture in large plastic vials with approx. 200-300 flies per vial and transferred to new vials every 2-3 days. All behavioral experiments were carried out within a timespan of the middle 9 hours of the light-phase (i.e. 1.5 hours after the light-cycle started and 1.5 hours before it ended). Flies used for imaging were raised in small vials with approx. 25-50 flies per vial and transferred to new vials every 2-3 days.

\subsubsection{Generation of transgenic flies}

In order to generate new transgenic fly strains, target DNA had to be amplified and cloned into vectors that could be injected into fly oocytes (see section 1.3 and [Rubin \& Spradling, 1982]). 


\section{Materials and Methods}

\subsubsection{Polymerase chain reaction (PCR)}

The DNA fragments encoding GCaMP3.0 and R-GECO1.0 were amplified by PCR [Saiki et al., 1988] for subsequent cloning into fly expression vectors. PCR was set up in a total volume of $50 \mu \mathrm{l}$ with $1 \mathrm{x}$ dreamTAG polymerase or Phusion polymerase buffer, $200 \mu \mathrm{M}$ dNTP, $100 \mathrm{pM}$ each primer, 10-200 ng template DNA and $0.5 \mu \mathrm{l}$ of dreamTAG or Phusion polymerase. The following primers were used:

For GCaMP3.0:

forward 5'-ATCAGATCTCAAAAAATGGGTTCTCATCATCATCATCATCATCATG-3' reverse 5'-ATCGCGGCCGCTTACTTCGCTGTCATCATTTTGTACAAACTCTTC-3'

For R-GECO1.0:

forward 5'-ATCAGATCTCAAAAATGGTCGACTCTTCACGTCG-3'

reverse 5'-AAGAAATGCGGCCGCCTACTTCGCTGTCATCATTT-3'

Both PCRs added a recognition site for the restriction enzyme Bglll to the 5'-end of the sequence and a recognition site for Notl to the 3'-end.

PCR was performed in the advanced Primus 96 thermocycler from PEQLAB (PEQLAB Biotechnologie GmbH; Erlangen, Germany). The following PCR protocols resulted in a sufficient yield of target DNA:

\begin{tabular}{|c|c|c|c|c|c|}
\hline \multirow{2}{*}{ Cycle step } & \multicolumn{2}{|c|}{ GCaMP3.0 } & \multicolumn{2}{c|}{ R-GECO1.0 } & \multirow{2}{*}{ Cycles } \\
& Temp. & Time & Temp. & Time & \\
\hline Initial denaturation & $94^{\circ} \mathrm{C}$ & $5 \mathrm{~min}$ & $98^{\circ} \mathrm{C}$ & $30 \mathrm{sec}$ & 1 \\
\hline Denaturation & $94^{\circ} \mathrm{C}$ & $30 \mathrm{sec}$ & $98^{\circ} \mathrm{C}$ & $30 \mathrm{sec}$ & \\
Annealing & $63^{\circ} \mathrm{C}$ & $90 \mathrm{sec}$ & - & - & 35 \\
Extension & $72^{\circ} \mathrm{C}$ & $30 \mathrm{sec}$ & $72^{\circ} \mathrm{C}$ & $60 \mathrm{sec}$ & \\
\hline Final extension & $72^{\circ} \mathrm{C}$ & $5 \mathrm{~min}$ & $72^{\circ} \mathrm{C}$ & $5 \mathrm{~min}$ & 1 \\
\hline Hold & $4^{\circ} \mathrm{C}$ & $\infty$ & $4^{\circ} \mathrm{C}$ & $\infty$ & \\
\hline
\end{tabular}




\section{Materials and Methods}

\subsubsection{Agarose Gel Electrophoresis}

PCR products and DNA fragments resulting from (analytical) restriction digests were visualized and separated by Agarose gel electrophoresis. 1\% agarose gel was prepared with TBE buffer. DNA was pre-mixed with $6 x$ loading dye and loaded on the gel together with the Generuler ${ }^{\mathrm{TM}} 1 \mathrm{~kb}$ DNA ladder, which was used for size determination of DNA fragments.

PCR product purification/Gel extraction PCR fragments were purified with PCR purification and gel extraction kits from Qiagen (Qiagen; Hilden, Germany). PCR products were either subjected to direct PCR purification or were separated on an $1 \%$-agarose gel, then the DNA fragments of correct size were extracted and purified according to the instructions from the protocol of the respective kit.

Restriction digest A digest with restriction endonucleases was performed on vector and insert DNA in order to generate sticky ends prior to ligation, as well as to analyze the plasmid DNA following MiniPrep. Restriction digests were set up in a total volume of $40 \mu \mathrm{l}$ with appropriate enzymes and buffers and incubated for 2-4 hrs at $37^{\circ} \mathrm{C}$.

Ligation of DNA-fragments into target vectors For the cloning of GCaMP3.0 under the control of the LexAop-promotor, TOPO ${ }^{\circledR}$ cloning with the Gateway ${ }^{\circledR}$ system was performed as described in the protocol provided with the kit. The GCaMP3.0 DNA from the PCR reaction was first cloned into the $\mathrm{pCR}^{\circledR} 8 / \mathrm{GW} / \mathrm{TOPO}{ }^{\circledR}$ vector and then subcloned into the pLOT $W$ vector for fly injection ([Diegelmann et al., 2008|) with an LR-reaction of Gateway ${ }^{\circledR}$ cloning as described in the protocol of the kit. During the LR reaction with the LR Clonase ${ }^{\circledR}$ II reaction mix, the gene in the $\mathrm{PCR}^{\circledR} 8 / \mathrm{GW} / \mathrm{TOPO}{ }^{\circledR}$ vector is exchanged with a $c c d \mathrm{~B}$ sequence in the target vector (pLOT W) via site-specific recombination [Hartley et al., 2000].

Ligation and bacterial transformation of One Shot ${ }^{\circledR}$ Mach 1 cells was performed according to the Gateway ${ }^{\circledR}$ protocol. For the cloning of R-GECO1.0, the PCR product was digested with Notl and Bglll and ligated into linearized destination vectors (pUASt and pCaSpEr:mb247). The subsequent ligation reaction was set up with $1 \mathrm{x}$ 


\section{Materials and Methods}

T4 DNA ligase buffer, $1 \mu \mathrm{l} \mathrm{T4}$ DNA ligase, $100 \mathrm{ng}$ vector and $~ 300 \mathrm{ng}$ insert. The ligation reaction was set up in a volume of $20 \mu \mathrm{l}$ and performed at room temperature for 1.5 hrs. Following ligation the vector was transformed into XL-Blue competent cells.

\subsubsection{Bacterial transformation}

Competent cells were thawed on ice. Ligation mix was added and cell suspension was incubated on ice for 30 minutes. The cells were heat-shocked in a waterbath at $42{ }^{\circ} \mathrm{C}$ for $45 \mathrm{sec}$ and immediately cooled on ice for $2 \mathrm{~min} .900 \mu \mathrm{l}$ of SOC medium was added and the cell suspension was incubated at $37^{\circ} \mathrm{C}$ for $1 \mathrm{hr}$. $100 \mu \mathrm{l}$ of the suspension was directly afterwards plated on agar-plates containing Ampicillin. The remaining suspension was centrifuged with $5000 \mathrm{rpm}$ for 5 minutes and $100 \mu \mathrm{l} \mathrm{su}$ pernatant plated on agar-plates. Finally, the rest of the supernatant was discarded, the pellet resolved in $100 \mu \mathrm{ISOC}$ and plated on agar-plates containing Ampicillin for selection. The plates were incubated over night at $37^{\circ} \mathrm{C}$.

\subsubsection{DNA isolation}

Bacterial colonies were picked up with a toothpick or a pipette tip from agar plates and resuspended in $5 \mathrm{ml}$ medium containing $100 \mu \mathrm{g} / \mathrm{ml}$ of the appropriate antibiotic. The bacterial culture was grown overnight at $37^{\circ} \mathrm{C} .2 \mathrm{ml}$ of the over night culture were used for plasmid isolation using the MiniPrep kit (Qiagen; Hilden, Germany) following the instructions in the protocol of the kit. When a higher amount of DNA was needed for sequencing and fly injection, midiPrep was performed with the MidiPrep kit (Qiagen; Hilden, Germany) following the instructions. For a midiPrep $200 \mu \mathrm{l}$ of miniPrep culture was inoculated into $200 \mathrm{ml}$ of LB medium supplemented with the appropriate antibiotic and the culture was grown overnight. Following plasmid isolation via mini- or midiPrep, DNA concentrations were determined with an ELISA reader and the Gen5 software from BioTek Instruments GmbH (Bad Friedrichshall, Germany) by measuring the light absorbance of the sample at 260 and $280 \mathrm{~nm}$. The sequence of the generated plasmids was verified by sequencing (performed by Eurofins MWG Operon; Ebersberg, Germany; Eurofins Headquarters in Huntsville, AL, USA)). DNA with a confirmed sequence was sent to BestGene Inc. (Chino Hills, 


\section{Materials and Methods}

CA, USA) to inject the vectors containing the target DNA into fly oocytes in order to generate transgenic flies [Rubin \& Spradling, 1982]. Vector maps of plasmids sent for injection were created with SpanGene ${ }^{\circledR}$ Viewer (GSL Biotech LLC; Chicago, IL, USA).

\subsubsection{Evaluation of transgenic fly lines}

After injection of the vectors into the germline cells by the company BestGene Inc. (Chino Hills, CA, USA), the expression of the transgenes was tested. The UAS and LexOp lines were combined with appropriate driver lines targeting the olfactory sensory neurons (Or83b). The mb247:R-GECO1.0 line did not need to be recombined and the expression was tested directly.

For each generated fly strain, several different versions were received. The insertion of the transgene via the p-elements took place in different regions of the genome. Therefore, the expression levels differ between the versions of the same genotype. In order to find the lines with the highest expression of the transgenes, the brains of five flies were dissected and the basal fluorescence levels measured. The measurements were conducted at a ZEISS Examiner2.1 with a 20x water objective with $100 \mathrm{~ms}$ illumination time. The basal fluorescence was averaged for each version and the functionality of the calcium sensor tested for the line with the highest expression. Therefore, the increase in fluorescence due to neuronal excitation with $\mathrm{KCl}$ was examined. The $\mathrm{KCl}$ measurements were performed by Ulrike Pech.

\subsubsection{Immunohistochemistry}

\subsubsection{Preparations of fly brains and stainings}

Wholemount preparations of adult fly brains including the thoracic ganglion were obtained from 5-10 day old flies. The animals were anesthetized on ice for a few minutes and the brains and thoracic ganglion dissected in Ringer's solution. The dissection of fly brains has been performed by Dr. Thomas Riemensperger. The brain preparations were subsequently fixed with $500 \mu \mathrm{l}$ of $4 \%$ PFA for $2 \mathrm{~h}$ on ice on a shaker. Afterwards, the preparations were washed 20-30 min in 500 $\mu$ PBT at room temperature to remove the remaining fixative. The brains were then blocked with 


\section{Materials and Methods}

$500 \mu \mathrm{l}$ PAT for $2 \mathrm{~h}$ at room temperature to decrease unspecific binding of the antibodies before an incubation period overnight at $4{ }^{\circ} \mathrm{C}$ in primary antibodies diluted in PAT. The $\alpha$-nc82-mouse antibody to label bruchpilot in the neuropils was diluted 1:5 whereas the $\alpha$-GFP-rabbit antibody was diluted 1:5000. On the next day, the preparations were washed three times in $500 \mu \mathrm{l}$ PAT for 20-30 min at room temperature to remove residual primary antibody and decrease unspecific labeling. After washing, the brains were blocked with $3 \%$ normal goat serum (NGS) in $500 \mu \mathrm{lPBT}$ at room temperature for $30 \mathrm{~min}$. Subsequently, the preparations were incubated for 2 hours at room temperature with the secondary antibodies diluted in $500 \mu \mathrm{l}$ of $3 \%$ NGS in PAT. The $\alpha$-rabbit-Alexa488 antibody was diluted 1:100 whereas the $\alpha$-mouse-Cy3 antibody was diluted 1:250. Afterwards, the brains were again washed three times for 20-30 min in $500 \mu \mathrm{l} \mathrm{PBT}$ to remove residual antibodies. The brains were then kept in PBS at $4{ }^{\circ} \mathrm{C}$ until they were mounted for confocal imaging.

\subsubsection{Confocal imaging}

The wholemount brain preparations including the thoracic ganglion were mounted with vectashield on microscope slides that were previously prepared with a cover of $1 \%$ agarose: $0.5 \mathrm{~g}$ of agarose was dissolved in $50 \mathrm{ml}$ TBE buffer by boiling in a microwave. A thin layer of the dilution was pipetted on a standard microscope slide and left to solidify before the fly brains with the attached thoracic ganglia were embedded and mounted in vectashield. The preparations were directly afterwards scanned with a confocal laser scanning microscope (Leica Microsystems $\mathrm{GmbH}$; Wetzlar, Germany) and a Plan-Apochromat 20x/0.7 NA objective (Leica Microsystems $\mathrm{GmbH}$; Wetzlar, Germany). Virtual slices of the preparations (z-stacks) were taken in two different wavelength (488 $\mathrm{nm}$ for Alexa488 and $550 \mathrm{~nm}$ for Cy3) with a thickness of $1 \mu \mathrm{m}$ per slice. The maximum projections of the obtained images were subsequently merged in Photoshop (Adobe Systems, Inc.; San Jose, CA, USA). Confocal imaging and subsequent image processing was done together with $\mathrm{Dr}$. Thomas Riemensperger. 


\section{Materials and Methods}

\subsubsection{Behavioral paradigms}

\subsubsection{Climbing assay}

The locomotor performance of flies was evaluated with a modified negative geotaxis assay [Ganetzky \& Flanagan, 1978; Feany \& Bender, 2000; Friggi-Grelin et al., 2003]. A $10 \mathrm{ml}$ serolocigal pipette (Carl Roth GmbH + Co KG; Karlsruhe, Germany) with an approximate length of $28 \mathrm{~cm}$ was divided into three parts. The lower part consisted of the lowest $7 \mathrm{~cm}$ region from the bottom of the pipette; the middle part was defined as $\sim 14 \mathrm{~cm}$ between the lower and the upper part and the upper part was the upper $7 \mathrm{~cm}$ region until the top of the pipette. The lowest centimeter of the tip of the pipette was filled with cotton to prevent flies from getting stuck and was subsequently concealed with parafilm.

Flies were anesthetized after hatching and collected in groups of 10 in plastic vials containing food medium. After 6-8 days, the flies were transferred into the pipettes without additional anesthesia and left to rest for ten minutes. During this phase the pipette was already placed in the experimental box (see 2.1.9) at $60-80 \%$ humidity. The temperature in the box was set to $32.0-33.0^{\circ} \mathrm{C}\left(24.5-25.5^{\circ} \mathrm{C}\right.$ for control) beforehand. After the resting period, the pipettes were tapped on the ground to let the flies fall to the lower part of the pipette. Subsequently, the flies had one minute to climb the walls of the pipette before the number of flies per compartment was counted. A performance index was calculated with the following formula:

$$
P I=\frac{1}{2} * \frac{n_{\text {total }}+\left(n_{\text {up }}-n_{\text {down }}\right)}{n_{\text {total }}}
$$

where $n_{\text {total }}$ is the total number of flies, $n_{u p}$ is the number of flies in the upper part and $n_{\text {down }}$ the number of flies in the lower part. The experiment was repeated three times and the average PI was used as a measurement of locomotor performance. Subsequent data analysis was done with Origin 8.5G (OriginLab; Northampton, MA, USA). Normal distribution was tested with the Shapiro-Wilk normality test. Differences in performance indices were tested for statistical significance with ANOVA and post hoc Bonferroni corrected t-tests. Visual presentation of the data was optimized with Adobe ${ }^{\circledR}$ Illustrator (Adobe Creative Suite 5, Adobe Systems, Inc.; San Jose, CA, USA). 


\subsubsection{Olfactory learning}
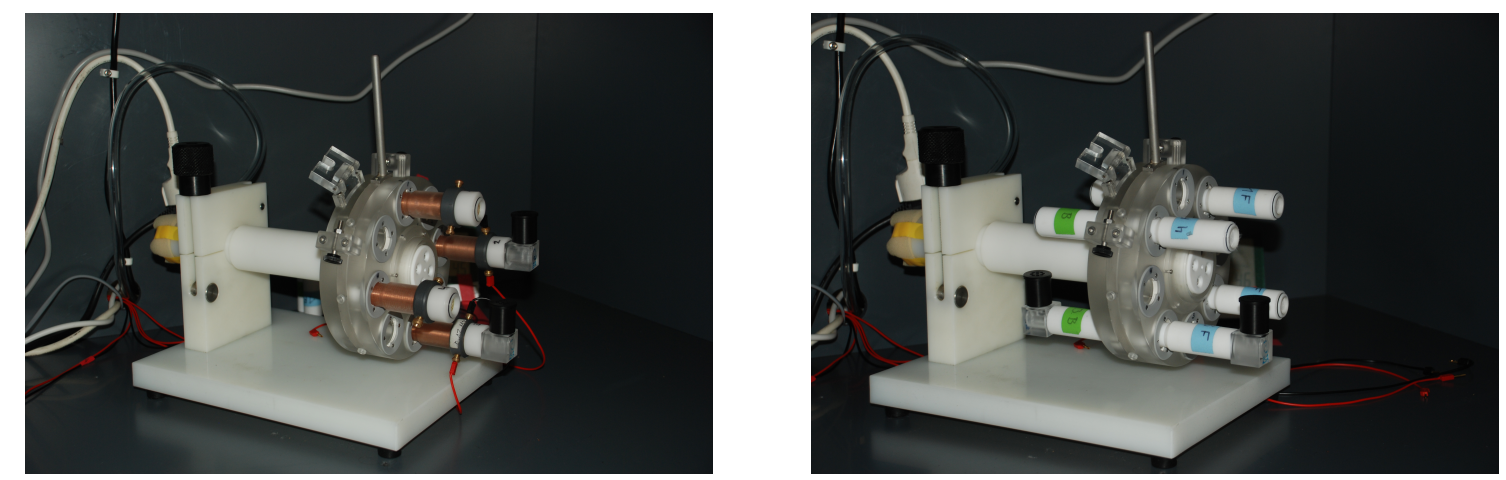

Figure 2.1. Photography of modified Tully-Quinn-Barrel for four parallel experiments. Different tubes can be attached to the machine: training tubes with an electrifiable copper grid were used during training (right side) whereas test tubes made out of polyethylene were attached during the test phase (left side). In both pictures the odor cups are visible and attached to the lower tubes.

Groups of about 100 flies (5-9d) were trained in an associative olfactory learning paradigm as introduced by Tully \& Quinn [1985] (see section 1.5). Four experiments were performed simultaneously in a modified learning apparatus described by Schwaerzel et al. [2002] (See figures 1.6 \& 2.1). A constant air flow of $\sim 167 \mathrm{ml} / \mathrm{min}$ in each tube assured a constant transport of odorant molecules inside the tubes.

All experiments were performed with Canton $\mathrm{S}$ wild type flies at $24-26{ }^{\circ} \mathrm{C}$ and $60-80 \%$ relative humidity if not stated otherwise. The used odorants were diluted in mineral oil in the following dilutions:

$\begin{array}{ll}\text { 3-Octanol } & 1: 750 \\ \text { 1-Octen-3-ol } & 1: 500 \\ \text { 4-Methylcyclohexanol } & 1: 400\end{array}$

$60 \mu \mathrm{l}$ of the diluted odorant were pipetted into an odor cup with $5 \mathrm{~mm}$ diameter and placed into the odor cubes which can be attached to the training and test tubes of the learning apparatus. Olfactory training started about one minute after transferring the flies into the tubes. Each odorant was presented for

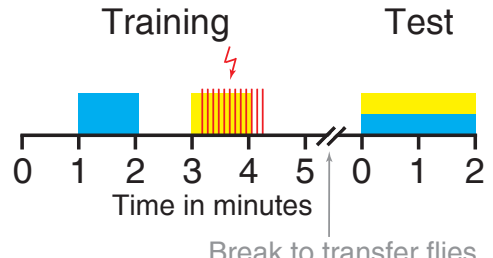

Figure 2.2. Time scheme for olfactory training. The training period lasts 5 minutes in which the flies are exposed to the CS+ and the CS-. The break between training and test phase, in which the tubes are exchanged and the flies are transferred inside the machine, ranged from 2 to $5 \mathrm{~min}$. The test phase lasted $2 \mathrm{~min}$. 


\section{Materials and Methods}

1 min with a 1 min break between odorant applications. One odorant (conditioned stimulus,+ CS + ) was temporally paired with 12 electric shocks of $90 \mathrm{~V}(1.25 \mathrm{sec}$ shock and $3.75 \mathrm{sec}$ inter pulse interval) direct current (DC) applied through an electrifiable grid covering the inside of the tubes. The second odorant was presented without shock (conditioned stimulus -, CS-). After another minute, the flies were transferred to a T-maze part of the apparatus with both odorants presented from each side. The change of tubes and transfer of the flies lasted 1-5 min before the 2 min test for odorant preference started (Figure 2.2). Subsequently, the flies in each tube were counted and a preference index was calculated by subtracting the number of flies on the side of the $\mathrm{CS}+\left(n_{C S+}\right)$ from the number of flies on the side of the CS- $\left(n_{C S_{-}-}\right)$, divided by the total number of flies $\left(n_{\text {total }}\right)$ :

$$
P I=\frac{n_{C S-}-n_{C S+}}{n_{\text {total }}}
$$

Negative Pls therefore represented an avoidance of the conditioned odorant, positive scores represent an attraction. Learning indices were calculated by averaging two reciprocal experiments in which each odorant was used once as CS+ and once as CS-. Odor preference was obtained with the same protocol by presenting an odorant or mineral oil instead of the CS+ and the CS-.

Subsequent data analysis was done with Origin 8.5G (OriginLab; Northampton, MA, USA). Normal distribution of the data was tested with the Shapiro-Wilk normality test. Differences in odorant preference, preference indices and learning indices were tested for statistical significance using either ANOVA with post hoc Bonferroni corrected t-tests or the Two-sample t-test. Visual presentation of the data was optimized with Adobe ${ }^{\circledR}$ Illustrator (Adobe Creative Suite 5, Adobe Systems, Inc.; San Jose, CA, USA).

The olfactory learning experiments with wild type flies were performed together with Moritz Hermann during his Bachelor Thesis [Hermann, 2011]. The Thesis was co-supervised by the PhD candidate and the work during data acquisition was shared. 


\section{Materials and Methods}

Shock avoidance was assayed in the T-maze. In contrast to the normal test situation, one of the arms of the maze consisted of the training tube. During the test of one minute, 12 electric shocks of 90V DC (1.25 sec shock and $3.75 \mathrm{sec}$ inter pulse interval) were administered to the training tube. The flies were placed in the elevator section in the middle of the Tully-machine. A subsequent counting of the flies on each side of the T-maze resulted in an avoidance index.

Odor preference was measured by placing the flies in the elevator section of the Tully-machine and a subsequent T-maze test. One arm of the maze was equipped with an odor cube with an odorant in the given dilution whereas the odor cube on the other arm was filled with the solvent, mineral oil. The flies had two minutes to decide which side of the T-maze to approach. After counting of the flies a preference index was calculated.

\subsubsection{Silencing of synaptic transmission during olfactory learning}

Inhibitory local interneurons in the antennal lobe were silenced using the shibire fly strain described by Kitamoto [2001] (see also section 1.3.2). Local interneurons of the LN1 type were targeted with the NP1227-GAL4 line [Seki et al., 2010; Sachse et al., 2007]. To block synaptic transmission in type I inhibitory local interneurons, $U A S-s h i^{t s} ;+; U A S-s h i^{t s}$ flies and $y, w^{-} ; \frac{N P 1227}{C y O} ;+$ were crossed. The offspring of this combination was tested in the learning paradigm at different temperatures. During the subsequent counting, the flies were sorted according to the expression of the CyO marker. The offspring without the CyO marker $\left(\frac{y, w^{-}}{U A S-s h i^{t s}} ; \frac{N P 1227}{+} ; \frac{+}{U A S-s h i^{t s}}\right)$ expressed the temperature sensitive dynamin mutant shibire in inhibitory local interneurons of the LN1 type. Offspring expressing the marker did not express shibire as no GAL4 was produced and was used as heterozygous control $\left(\frac{y, w^{-}}{U A S-s h i^{t s}} ; \frac{C y O}{+} ; \frac{+}{U A S-s h i^{t s}}\right)$. The $y, w^{-} ; \frac{N P 1227}{C y O} ;+$ parental line served as a control for the GAL4-driver.

Prior to the training procedure described above, all flies were transferred into empty vials and kept at the respective temperature next to the training apparatus for $\sim 10$ min to ensure complete blocking of synaptic transmission. Type II inhibitory local interneurons were targeted with the NP2426 GAL4-enhancer-trap line (NP2426; +; +). 


\section{Materials and Methods}

In order to get appropriate heterozygous genetic controls for the combination of $N P 2426 ;+;+$ and $U A S-s h i^{t s} ;+; U A S-s h i^{t s}$, the parental lines were crossed with $\mathrm{W}^{1118}$ and the offspring used during the experiments.

\subsubsection{In-vivo calcium imaging}

\section{Fly preparation and image acquisition}

Flies expressing the genetically encoded calcium sensor GCaMP3.0 [Tian et al., 2009] were immobilized for $\sim 5 \mathrm{~min}$ on ice. A single female fly was subsequently placed in the self-made fly holder and fixed with adhesive tape. A drop of Ringer's solution is put on the tape above the head to prevent the brain from drying out

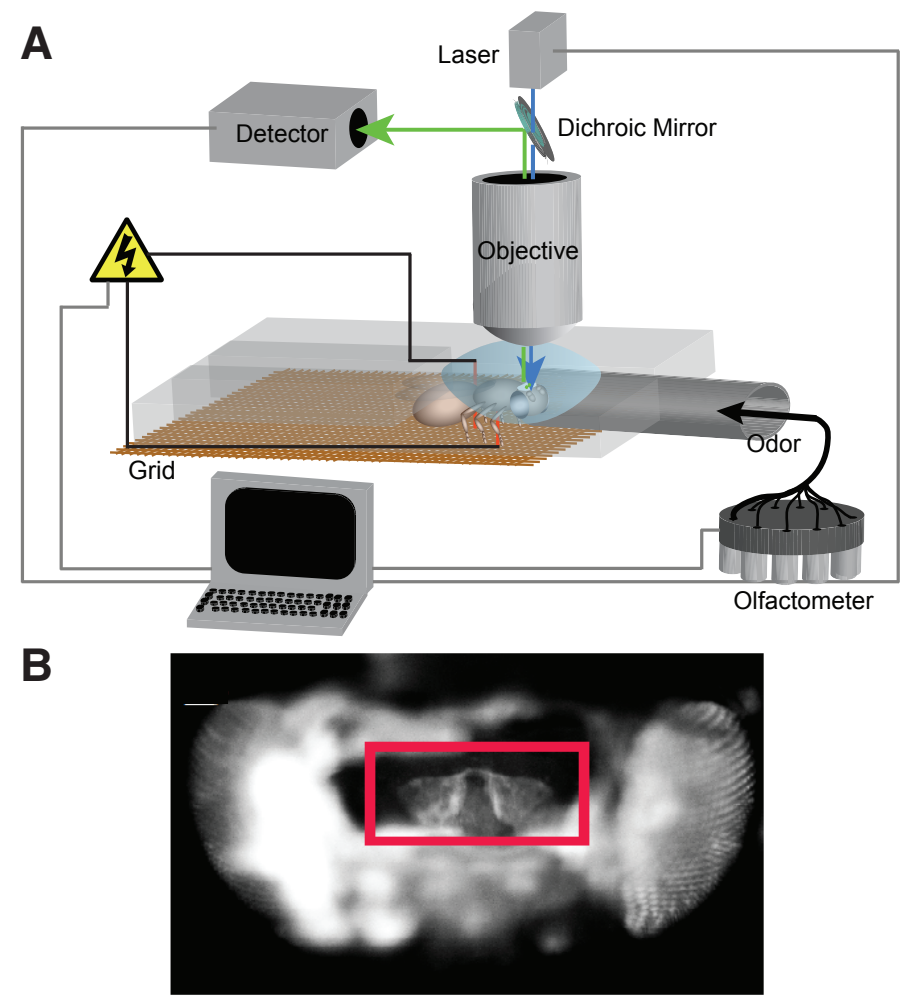

Figure 2.3. Fly preparation for optical imaging.

(A) The fly was placed in a self-made fly holder consisting of a microscopy slide, a metal grid and sticky tape. A window was cut into the cuticle of the head to get visual access to the brain. Odor presentation was carried out by a computer controlled olfactometer. Possible application of an electric shock during conditioning experiments was also computer controlled and conveyed via copper wires that touched the flies' legs, thorax and abdomen. Images were acquired with a 2-photon microscope to measure the fluorescent changes following changing calcium concentrations.

(B) Expression of the fluorescent calcium sensor GCaMP3.0 can be visualized under the microscope. Here, the antennal lobes are labeled.

Figure kindly provided from $\mathrm{T}$. Riemensperger with modifications 


\section{Materials and Methods}

during the preparation. A piece of a razor blade and a blade holder was used to cut a rectangular window in the tape and the cuticle of the fly head was removed with fine forceps under the microscope. The window was located between the ocelli and the compound eyes. Fat bodies were removed and subsequently the hemolymph of the head sucked away with a small piece of tissue. Directly afterwards, warm low melting agarose solution was filled in the fly head to fix the brain and prevent movement.

The calcium activity in the antennal lobes or the mushroom body was measured with a 20x water immersion objective and an image acquisition rate of $5 \mathrm{~Hz}$. An excitation wavelength of $920 \mathrm{~nm}$ and a band-pass filter for GFP emission was used during image acquisition. After measuring the calcium activity, a series of images as optical slices (z-stack) was recorded in order to identify glomeruli or lobe regions during data analysis. Fly preparation and imaging were performed by Shubham Dipt.

Experimental design During each odor stimulus, 85 images were obtained. The stimulus was presented for $2 \mathrm{sec}$ ( 10 images). Image acquisition started $5 \mathrm{sec}$ (25 images) before the odor onset in order to record a baseline and ended $10 \mathrm{sec}$ after the stimulus offset (50 images). Between each odorant presentation was a break of $20 \mathrm{sec}$ to remove residual odorant molecules from the olfactometer and to ensure that the calcium level drops back to baseline. Thereby, each stimulus bout lasted 37 sec.

Measurements in the antennal lobe consisted of a presentation of mineral oil as a control and a subsequent presentation of each of the three odorants (1-Oct, 3-Oct and $\mathrm{MCH}$ ) to determine the glomerular pattern activated by the odorants. The calcium activity in the antennal lobe was measured in the different focal planes to cover the activation pattern of various glomeruli.

Measurements in the mushroom body included two phases: a pre-training and a post-training phase. During both phases, the three odorants were presented three times in a pseudo-randomized order with a $20 \mathrm{sec}$ pause between each presentation. After the pre-training phase and a $30 \mathrm{sec}$ pause, the fly was trained in a protocol similar to the behavioral training: the CS+ (1-Oct or 3-Oct) was presented together with an electric shock (12 pulses of $1.25 \mathrm{sec}$ with $90 \mathrm{~V} \mathrm{DC}$ ). After a $60 \mathrm{sec}$ break, the CS- was presented for $60 \mathrm{sec}$. The post-training phase started subsequently after 


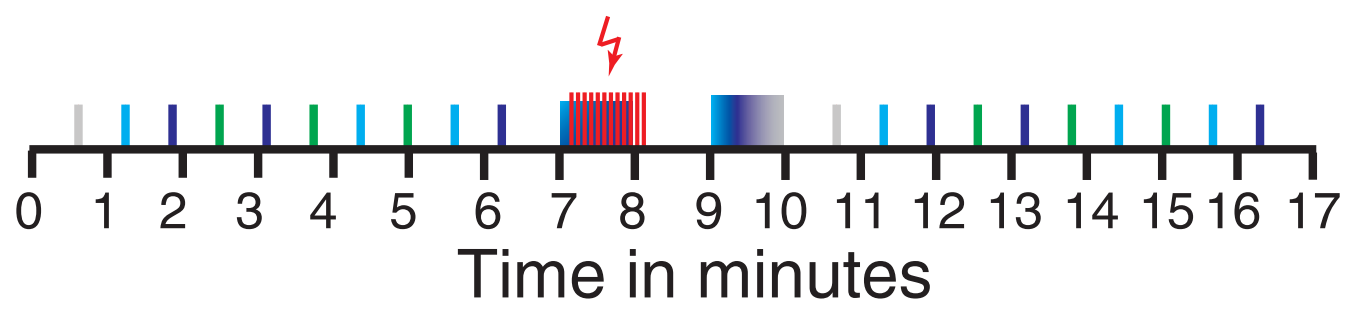

Figure 2.4. Scheme of the experimental design for mushroom body imaging. In the pre-training phase, mineral oil is presented as a first stimulus (gray). Subsequently, the three odorants 1-Oct (light blue), 3-Oct (dark blue) and MCH (green) are presented in a pseudo-randomized order for $2 \mathrm{sec}$ with an interstimulus interval of $35 \mathrm{sec}$. During the training phase, the $\mathrm{CS}+$ was paired with a negative reinforcement (12 electric pulse of $1.25 \mathrm{sec}$ with $90 \mathrm{~V} \mathrm{DC}$ over $60 \mathrm{sec}$ ). Afterwards, either the CSor mineral oil as a replacement of the CS- was presented for $60 \mathrm{sec}$. The following post-training phase was equal to the pre-training stimulation.

another $30 \mathrm{sec}$ pause (Figure 2.4).

Four different experimental groups were tested: Group A consisted of flies trained in an absolute training paradigm (the CS+ was 1-Oct and the CS- mineral oil). Group B comprised flies trained in a differential training paradigm (1-Oct served as CS+ and 3-Oct as CS-). Group C was absolute training of 3-Oct with mineral oil as CS, whereas group D consisted of flies trained differentially with 3-Oct as CS+ and 3-Oct as CS-. These groups were combined during the data analysis depending on the received training paradigm (absolute or differential).

\section{Data analysis}

The evaluation of the imaging data was performed together with Shubham Dipt. During the analysis, the following programs have been used: MetaMorph ${ }^{\circledR}$ (Molecular Devices Corporation; Sunnyvale, CA, USA), Excel (Microsoft; Redmond, WA, USA), Origin 8.5G (OriginLab; Northampton, MA, USA), self-written plugins for ImageJ [Abràmoff et al., 2004; Schneider et al., 2012], MatLab (The MathWorks; Natick, MA, USA) and the open source software R [R Core Team, 2012]. 


\section{Materials and Methods}

Definiton of regions of interest In the antennal lobe, the regions of interest consisted of single glomeruli that respond to the presented odorants with an increase in the calcium concentration. Individual glomeruli were identified with the optical slices of the antennal lobe and the help of a 3D model of the glomerular structure of the antennal lobe [Laissue et al., 1999] available online in the fly brain atlas at http://www.flybrain.org [Armstrong et al., 1995]. Comparisons of obtained odorant evoked responses with previous studies were performed with the help of the Database of Odorant Responses (DoOR) available online at http://neuro.unikonstanz.de/DoOR [Galizia et al., 2010].

The different lobes represented the regions of interest in the mushroom body. Additionally, the $\gamma$-lobe was further separated after Tanaka et al. [2008]. The described $\gamma$-lobe regions 1, 2 and 3 were combined as the calcium signal in the $\gamma$-lobe was mainly observed in these regions. An anatomical differentiation between the three regions was not possible. The optical slices of the mushroom body in varying focal planes were used to identify the lobe regions.

Analysis of the activity in the antennal lobe For the analysis of the images obtained in the antennal lobe, the data was preprocessed with ImageJ to align the gathered images to reduce movement artifacts with a custom written plugin based on the existing TurboReg plugin [Thévenaz et al., 1998]. Subsequently, MetaMorph ${ }^{\circledR}$ was used to define a region of interest and export the average fluorescent intensities as gray values at each time point to Excel. Five image before the onset of each stimulus were averaged to obtain a basal fluorescence value $\left(F_{0}\right)$. The difference in intensity during the stimulus $(\Delta \mathrm{F})$ was calculated by subtracting $F_{0}$ from the fluorescence intensity value of each image $\left(F_{i}\right)$. The difference in intensity at each time point was subsequently divided by the basal intensity to normalize for different expression levels of GCaMP3.0 in individual flies: $\quad \frac{\Delta F}{F_{0}}=\frac{F_{i}-F_{0}}{F_{0}}$ The $\frac{\Delta F}{F_{0}}$ values were copied to Origin $8.5 \mathrm{G}$ and plotted as a fluorescent change in percent over time. False-color coded images for example flies were obtained with ImageJ by subtracting the image before the stimulus onset from the image at the peak of the intensity difference and divided by basal fluorescence $\left(\frac{\Delta F}{F_{0}}\right)$. Noise was decreased with a mean filter with a 5 pixel range. The lower $50 \%$ of the signal were 


\section{Materials and Methods}

cut off so that only areas are shown that have an increase in signal intensity of more than $50 \%$ of the maximal change. The false color images were subsequently superimposed on the gray scale image that was used as the baseline.

\section{Pixel-based correlation between images in the mushroom body}

The data obtained during two-photon calcium imaging in the mushroom body horizintal lobes had to be preprocessed before any information about the similarity of two odorants could be extracted. Therefore, the raw data was first aligned with a MatLab program for image alignment in order to remove movement artifacts [Guizar-Sicairos et al., 2008]. A Kalman-Filter [Kalman, 1960] was subsequently applied with an ImageJ plugin to remove noise without losing spatial information. False-color coded images were obtained with ImageJ as described above with the only difference that a mean filter with a 1 pixel range was applied to decrease the loss of spatial information.

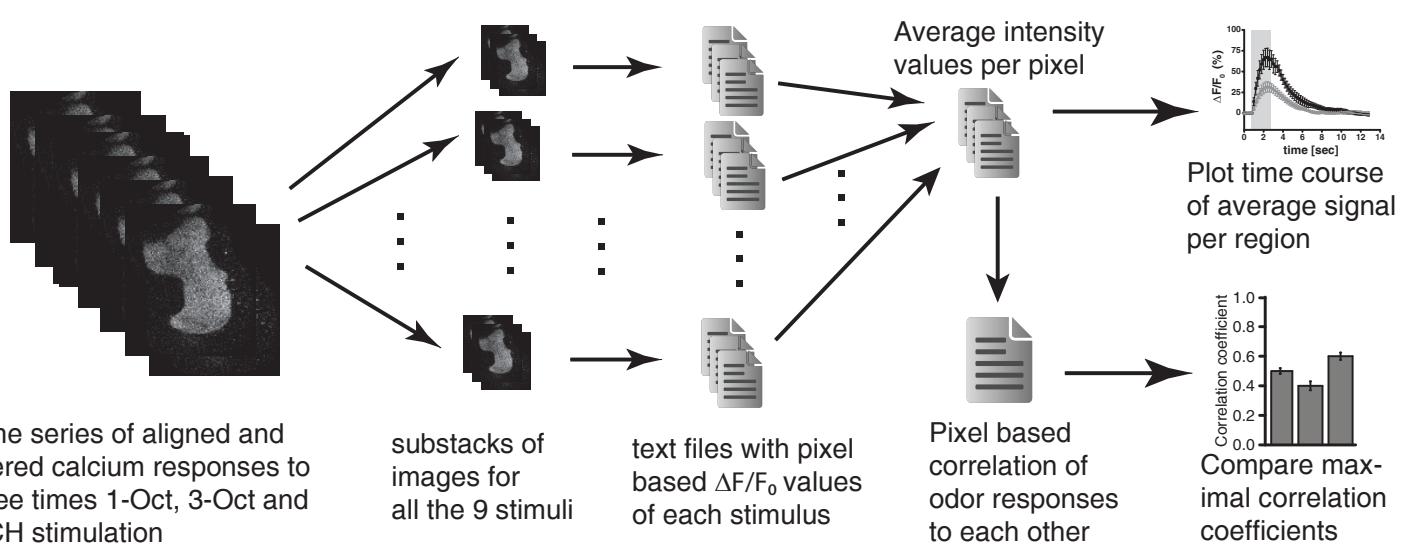

Figure 2.5. Scheme of picture analysis. Time series of the preprocessed (aligned and Kalman-filtered) data were processed with MatLab and ImageJ. The complete dataset of 9 measurements (three repetitions of each odorant) was divided into different stacks corresponding to the distinct odorant presentations. The change in signal intensity $\left(\frac{\Delta F}{F_{0}}\right)$ was calculated and the three presentations of the same odorant combined by averaging. From these pixel values, the average intensity per region was plotted and the correlation between the different odorants computed. The maximal correlation coefficients were used to compare the similarity of the activation patterns evoked by the different odorants. 


\section{Materials and Methods}

In order to compare the signals of different flies in the mushroom body lobes, the aligned images of a measurement were further processed with ImageJ and MatLab (see figure 2.5). The regions of interest were chosen as described above and the measurements cropped to create distinct datasets for each of the chosen regions for the pixel-based analysis. A custom written ImageJ plugin was used to split each set of images into subgroups containing all images of one odorant stimulus. The images were afterwards processed in MatLab to obtain $\frac{\Delta F}{F_{0}}$ intensity changes for each pixel and the values were exported as a text file. The average of all three odorant stimulations of a measurement was calculated for further processing. In order to evaluate the changes in fluorescence intensity, these values were plotted as time courses. Additionally, the similarity of the odorant evoked activation patterns was investigated by calculating the pixel based correlation coefficient (pearson correlation) between two odorants during the stimulation. The maximal correlation coefficient for each odorant pair was extracted for each fly. The correlation coefficients from all measured flies before the training were pooled to estimate the innate similarity of the three odorants to each other. Subsequently, the flies were separated in two groups depending on the training they were receiving (see above) and effects on odorant similarity before and after training observed. 


\section{Results}

\subsection{Establishing the olfactory learning paradigm in the laboratory}

Research on the generalization and discrimination of similar odorants and the effects of different training paradigms on olfactory perception on a behavioral level could only be performed after establishing the olfactory learning paradigm in the laboratory. Therefore, various experiments have been performed in order to define the conditions that resulted in stable learning of the flies. The Tully-machine (see figure 2.1) was placed in a box built by the university workshop (see section 2.1.9) to keep the environment as constant as possible. Stable temperature and humidity conditions had to be found by optimizing the use of humidifiers and heating devices. The Tully-machine itself had to be tested for constant functioning to minimize variations in side preference of the animals without any stimulus. The situation at the beginning of the thesis comprised different preference indices for the four tubes of the Tully-machine (Figure 3.1 A). The tubes had to be adjusted in their size and the connection frames of the machine tightened to minimize tilting of the tubes. The tightness of the tubes also influenced the air flow that passed by the odor containers and therefore transports the odorant molecules through the tubes. After calibration, the four tubes showed no preference to any side (Figure 3.1 B). Nonetheless, all experimental groups during all experiments were tested in each of the four arms of the machine to avoid any biased results. The influence of different light conditions also had to be considered and measured (Figure 3.1 C). If normal room light was the only light source, the flies did not show an equal distribution to both sides of the T-maze without any stimulation. Therefore, the behavior of the flies during different light conditions was investigated. In complete darkness, the flies distributed equally 

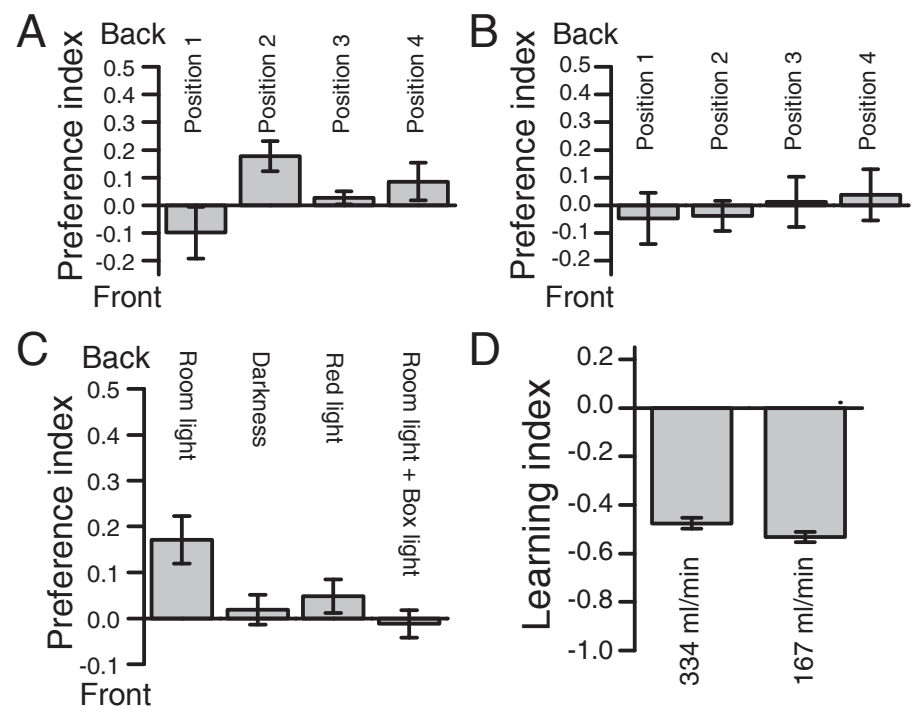

Figure 3.1. Calibration of the Tully-machine. In order to calibrate the setup, approx. 100 flies were placed in the machine and their distribution in the T-maze counted. (A) The four positions of the test tubes within the machine showed a varying preference of the flies for one of the sides of the T-maze without any stimulus before they were calibrated. (B) After calibration of the tightness of the tube connectors, the flies were distributing equally to both sides of the

T-maze in all four test tubes. (C) The light conditions in the experimenting room influenced the behavior of the flies. An equal distribution to both sides of the machine could only be achieved in darkness, under red light conditions and with an illumination of the box from the inside together with the room light. (D) A decrease of the air flow down to $167 \mathrm{ml} / \mathrm{min}$ did not result in a decreased learning index for differential training of $\mathrm{MCH}$ and 3-Oct.

which suggested an influence of the light. However, performing experiments in total darkness was not possible. Therefore, red light as the only light source and a combination of a white light source in the experimental box together with normal room light was considered. During both conditions, the flies did not show any preference for neither of the sides. The combination of a light source within the experimental box and the normal room light source was chosen for all further experiments.

An optimal rate of the air flow through the machine had to be determined in order to "transport" the odorant molecules from the odor cubes to the flies without irritation of the animals. Tully \& Quinn [1985] were using an air flow of $660 \mathrm{ml} / \mathrm{min}$ to train the flies and $1320 \mathrm{ml} / \mathrm{min}$ during the test, whereas the air flow during both phases was $750 \mathrm{ml} / \mathrm{min}$ in the experiments from Schwaerzel et al. [2003]. Therefore, the initially very strong air flow of $3667 \mathrm{ml} / \mathrm{min}$ in each tube of the machine had to be reduced. The university workshop helped to integrate a lever behind the air pump of the machine to regulate the air flow on a very fine scale. In order to decrease the physical irritation of the flies further, an even lower air flow was tested during learning 


\section{Results}

experiments. 3-octanol (3-Oct) and 4-methylcyclohexanol $(\mathrm{MCH})$ were trained reciprocally as described in section 2.2.4.2 with odorant dilutions of 1:20 for 3-Oct and $1: 5$ for $\mathrm{MCH}$. No difference in the resulting learning indices between an air flow of $367 \mathrm{ml} / \mathrm{min}$ and $167 \mathrm{ml} / \mathrm{min}$ could be observed (Figure 3.1D). Therefore, an air flow of $167 \mathrm{ml} / \mathrm{min}$ was used for all following experiments. However, the learning indices that where reached in the lab during that time were significantly lower than what could be found in the literature [Pitman et al., 2009]. After various experiments with different odorant concentrations (data not shown), the husbandry of the flies was altered. Initially, flies were kept in mass culture in glass vials in boxes in the lab. After transferring the boxes with the vials into incubators with constant and controlled conditions ( $60 \%$ humidity, $25^{\circ} \mathrm{C}$ and a $12 \mathrm{~h} / 12 \mathrm{~h}$ light/dark cycle), the learning indices could be improved. Differential olfactory learning for each of the tested odorant combinations of 3-octanol (OCT, dilution 1:20), 4-methylcyclohexanol ( $\mathrm{MCH}$, dilution 1:5) and benzaldehyde (BA, dilution 1:33) resulted in stable learning indices in line with the literature (Figure 3.2). These results provided the basis for further conditioning experiments with varying learning paradigms or transgenic flies.

Figure 3.2. Olfactory conditioning resulted in stable learning indices. After the machine was calibrated and the fly maintenance was optimized, the results of the olfactory conditioning experiments were improved. Temporal pairing of the presentation of one odorant (CS+) with electric shocks whereas the other odorant (CS) is presented without punishment resulted in an avoidance of the punished odorant by the flies in the T-maze. Reciprocal experiments during which each odorant served once as CS+ and once as CS- minimized odorant specific bias. The learning index is calculated by the mean of two reciprocal experiments. All three odorant combinations tested resulted in similar learning scores. $\mathrm{n}=16$ each; mean \pm SEM

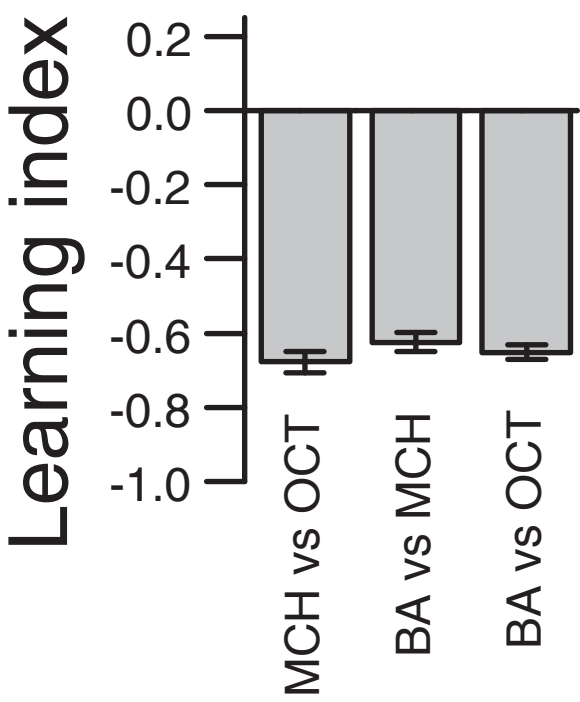




\section{Results}

\subsection{Sensory preconditioning experiments}

A first approach towards a better understanding of the mechanisms underlying olfactory perception in general and similarity of odorants in particular was to investigate sensory preconditioning. The presentation of a combination of two distinct stimuli preceding a training procedure that reinforces one of the two stimuli is called sensory preconditioning [Pavlov, 1927; Brogden, 1939; Kimmel, 1977; Rescorla, 1980; Müller et al., 2000; Brembs \& Heisenberg, 2001]. As a read-out, the response towards the second, not-reinforced, stimulus is tested. Previous experiments on sensory preconditioning in flies with visual stimuli showed their ability to fulfill the task by forming an association between a color and a simultaneously presented pattern [Brembs \& Heisenberg, 2001]. During crossmodal preconditioning, two sensory stimuli from different modalities are presented in the preconditioning phase. Association of an olfactory and a visual stimulus during preconditioning could be observed in Drosophila [Guo \& Guo, 2005] and the cricket Gryllus bimaculatus [Matsumoto et al., 2013]. Purely olfactory sensory preconditioning could be shown in the honey bee (Apis melifera) [Müller et al., 2000]. Therefore, an experiment was designed in order to test olfactory preconditioning in the fly: during the preconditioning phase, the flies were subjected to a binary mixture of two distinct odorants $(A B)$. In the training phase, one of the odorants $(A)$ was paired with an electric shock whereas a novel odorant $(C)$ was presented without reinforcement. During the subsequent T-maze test, the flies could decide to approach either odorant B or odorant C (Figure $3.3 \mathrm{~A}$ ). The control group comprised of flies that were treated exactly the same as the test group with the only difference of a presentation of mineral oil instead of the odorant mixture during the preconditioning phase. The control group therefore was not subjected to sensory preconditioning.

The paradigm includes three distinct olfactory stimuli. Therefore, three odorants were chosen that have very different chemical structures and were shown to be perceived as dissimilar by the flies [Niewalda et al., 2011]: 3-octanol, 4-methylcyclohexanol and benzaldehyde. To exclude any bias due to the odorant identity, each of the odorants was used as odorant A, B and C in a distinct set of experiments. During each of these subsets of experiments, no difference between the test group and the control group could be observed. A combination of the different 


\section{Results}

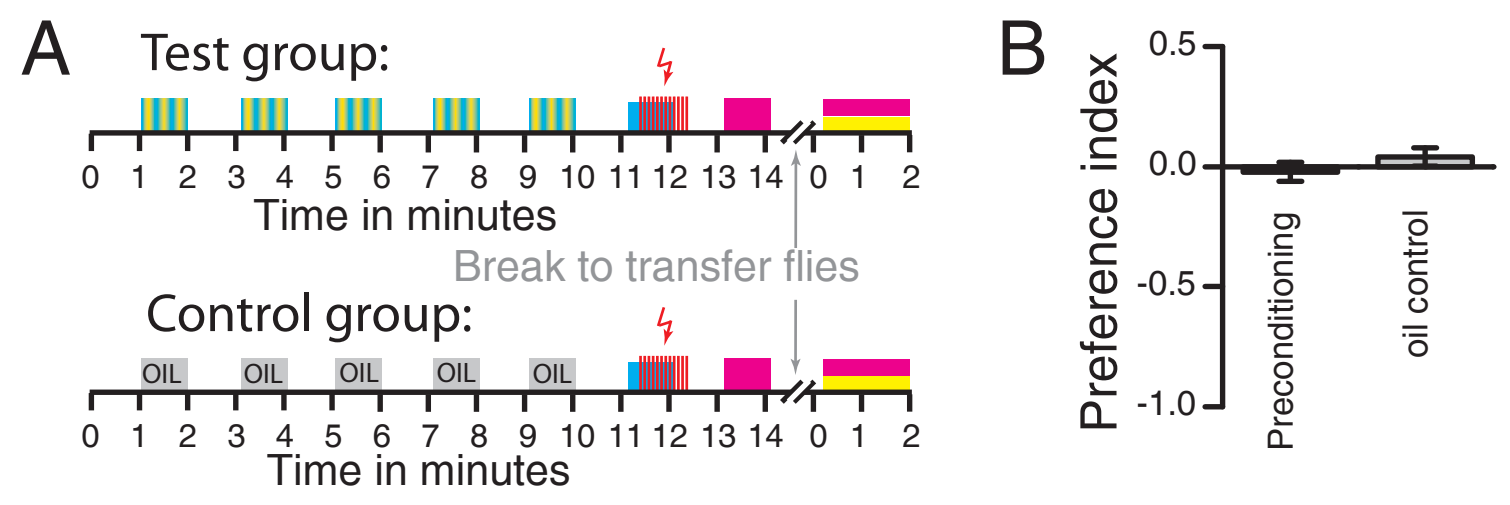

Figure 3.3. Test for the flies' ability for olfactory sensory preconditioning

(A) During the preconditioning phase, a binary mixture of two odorants ("yellow" and "blue") was presented to 100 flies in the tubes for five times for one minute. In the subsequent training phase, one of the two odorants ("blue") served as a CS+ and a novel odorant ("magenta") was presented as a CS-. During the test, the flies had to decide between "magenta" and "yellow" in a T-maze. In the control situation, the flies were subjected to mineral oil during the preconditioning phase. (B) The results of the experiments with each of the three used odorants (benzaldehyde, 3-octanol and methylcyclohexanol) and an alternation of their role (yellow, magenta or blue) led to no differences between the control group and the flies that were subjected to sensory preconditioning. Both groups did not show any learning but an equal distribution of the flies in reciprocal experiments.

$\mathrm{n}=109$ each; mean \pm SEM

subsets of experiments, which excludes all bias due to odorant identity, showed an equal distribution of the flies to both sides of the T-maze in the test group and the control group (Figure 3.2 B). Whereas this result was expected for the control group in which one odorant was paired with an electric shock and a completely different one was tested in the T-maze, the test group showed an unexpected behavior. If an association between the two odorants in the mixture would have been formed, the flies would have shown an avoidance of the odorant presented during the preconditioning phase. Therefore, it can be concluded that the olfactory preconditioning paradigm, with the odorants and the dilutions that were used during this particular experiment, does not lead to an association of the two presented odorants in the mixture.

Consequently, a different approach to investigate perceptual and physiological changes in odor distance and similarity after learning had to be considered. The generalization of distinct but similar odorants and a learned discrimination due to 


\section{Results}

the reinforcement of one of the two odorants was the alternative chosen for this study. In particular, it was investigated whether different training paradigms have an influence on the generalization and discrimination of similar odorants. Absolute training comprises of the association of an odorant $A$ with an electric shock (CS+) and a subsequent test for avoidance of the odor. Differential training on the other hand includes a CS-: while the presentation of odorant $A$ is associated with an electric shock (CS+), another odorant B is presented without reinforcement (CS-). The test situation also includes both odorants and the subjects have to decide which odorant to approach or avoid, respectively. As a prerequisite to study the effects of absolute and differential training on generalization and discrimination, the response of the flies to the two used similar odorants was observed. If the flies generalize between two odorants, they do not show a different behavior towards the two odorants. Experiments were performed to test the generalization of two distinct but structurally similar odorants 3-octanol (3-Oct) and 1-octen-3-ol (1-Oct). 4-methylcyclohexanol $(\mathrm{MCH})$ was used as a dissimilar control odorant (See figure 1.9).

\subsection{Determining odorant concentrations}

Preliminary experiments were conducted to figure out odorant dilutions that evoke equal responses of the flies. A similar naïve response of the flies towards all three odorants is necessary to exclude possible intrinsic preferences towards one of the odorants. Otherwise, intrinsic preferences towards an odorant could alter the responses of the flies after the training procedure and therefore change the behavior in the choice situation.

Responses of the flies towards different dilutions of the three odorants were tested with respect to three questions. The first question deals with the ability of the flies to learn the association of the odorant with electric shocks? (learnability). Can the flies learn to avoid a particular odorant if it is paired with electric shocks? Therefore, the learnability of different odorant dilutions was assessed in a first step. The dilutions should be chosen in a way that the learnability of all three odorants is similar. Hence, each of the odorants was tested in dilutions of 1:50, 1:100, 1:500 and 1:1000. The results of this experiment are visualized in figure 3.4. The learning scores show a similar learnability of all three odorants with only small differences. 


\section{Results}
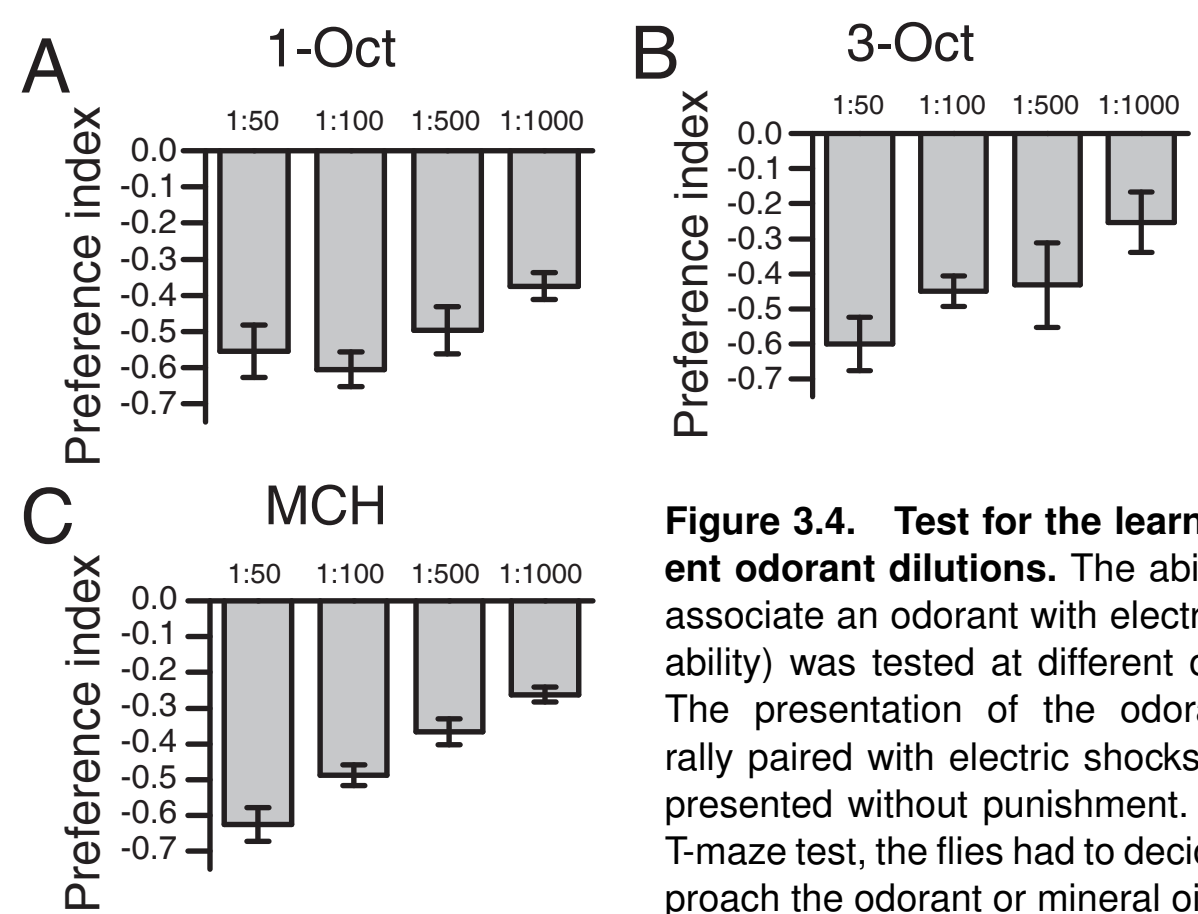

Figure 3.4. Test for the learnability of different odorant dilutions. The ability of the flies to associate an odorant with electric shocks (learnability) was tested at different odorant dilutions. The presentation of the odorant was temporally paired with electric shocks. Mineral oil was presented without punishment. In a subsequent T-maze test, the flies had to decide whether to approach the odorant or mineral oil. (A) The learnability of 1-octen-3-ol(1-Oct) increased slightly in the observed range of dilutions. (B) 3-octanol (3-Oct) showed a weak learnability in a high dilution of 1:1000. An increase of the concentration resulted in a preference index comparable to 1-Oct (C) The learnability of 4-methylcyclohexanol $(\mathrm{MCH})$ rises with increasing concentrations in the observed range. $\mathrm{n}=8-16$; mean \pm SEM

In order to see improvement of learning as well as decreased learning in the main experiments, odorant dilutions in the intermediate learnability range were chosen. The second aspect includes the behavior of the fly when the choice between two odorants at varying dilutions was given: which odorant is preferred by the flies when given the choice in a T-maze situation? The odorant dilutions had to be chosen in a way that the flies do not show any innate preference for one of the odorants.

As a result from the previous experiment concerning the learnability, the response of the flies towards 1-Oct and 3-Oct in the same dilution of 1:500 was tested without any reinforcement. Surprisingly, the flies did not distribute equally to both sides of the T-maze but approached 3-Oct with a preference index of 0.2. Hence, the dilution of 1-Oct was decreased to 1:400. With odorant dilutions of 1:400 for 1-Oct and 1:500 for 3-Oct, an equal distribution in the T-maze could be observed (Figure 3.5 A). 


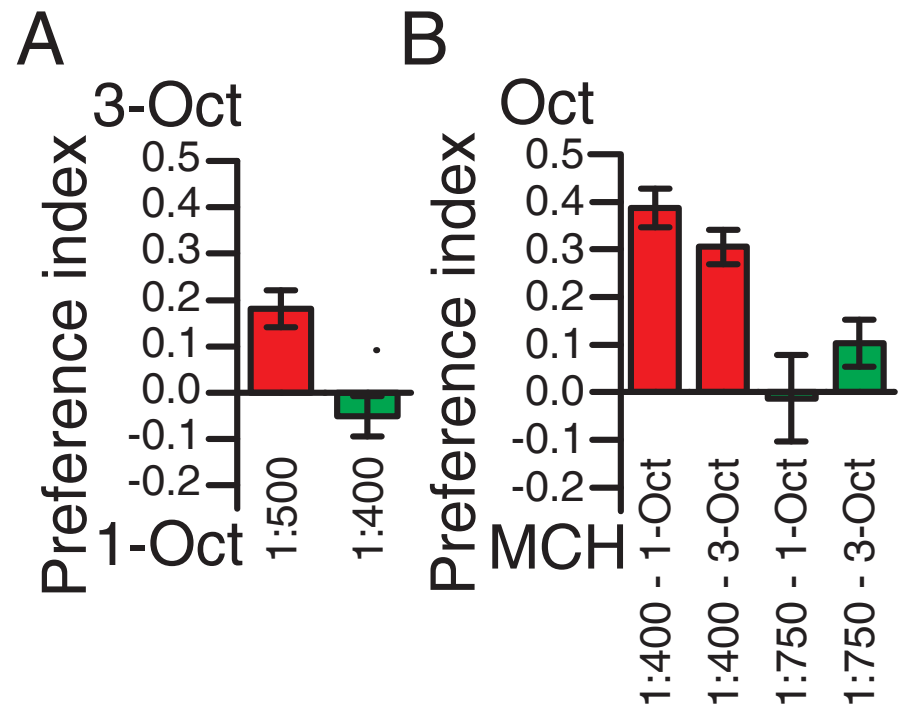

Figure 3.5. Naïve responses towards odorants in changing dilutions. Approx. 100 flies were subjected to a T-maze test and had to decide whether to approach one of the two presented odorants. (A) 3-Octanol in a 1:500 dilution was compared to 1-octen-3-ol in a dilution of $1: 500$ or $1: 400$. A preference towards 3-Oct could be observed with 1-Oct in a dilution of 1:500, but was not present after diluting 1-Oct to 1:400.

(B) 3-Oct (1:400) and 3-Oct(1:500) were tested against methylcyclohexanol in a dilution of 1:400. The strong preference towards OCT could be decreased by changing the $\mathrm{MCH}$ dilution to $1: 750$.

$\mathrm{n}=4-8$ each; mean \pm SEM

A next step was to investigate the behavior towards $\mathrm{MCH}$ with either 1-Oct or 3-Oct in the determined dilutions. Initially, a dilution of 1:400 for $\mathrm{MCH}$ was chosen. However, the flies showed a preference towards 1-Oct and 3-Oct. Thus, the dilution of $\mathrm{MCH}$ was increased to 1:750. The combinations of dilutions of 1:500 for 3-Oct, 1:400 for 1-Oct and 1:750 for $\mathrm{MCH}$ resulted in an almost equal distribution of the flies when subjected to a T-maze choice between the odorants (Figure 3.5 B). As a consequence, these odorant dilutions were used for all upcoming experiments.

The third question addresses the innate response of the flies towards a single odorant without any reinforcement: how do the flies respond when they have the choice between the odorant and mineral oil in a T-maze situation? The learnability of an odorant does not necessarily correlate with the innate response towards this odorant [Saumweber et al., 2011]. Therefore, the behavior of the flies towards the three odorants in the previously chosen dilutions was observed. The flies equally approached the three odorants when they were asked to choose between the odorant and the solvent mineral oil (Figure 3.6 A). No preference was observed between an empty odor cup (AIR) and mineral oil. If the presentation of mineral oil was associated with an electric shock, the flies tend to increase their approach 


\section{Results}

towards the odorant or an empty odor cup (AIR) but no significant difference to the situation without an electric shock could be observed (Figure 3.6 B). On the contrary, the flies avoided each of the odorants after pairing the presentation of the odorant with an electric shock (3.6 C). The avoidance of the odorants was comparably similar for each of the odorants. The results of the association of

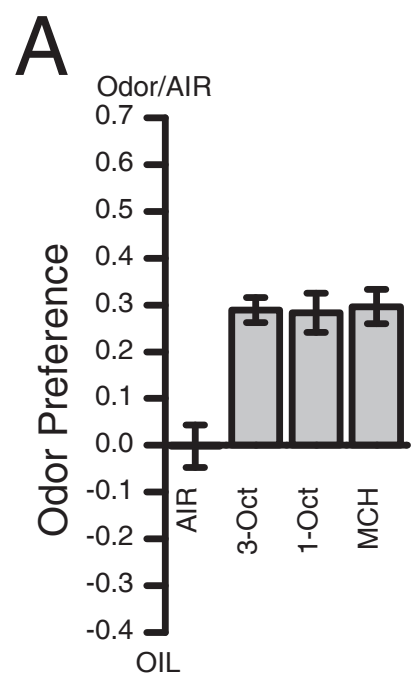

C

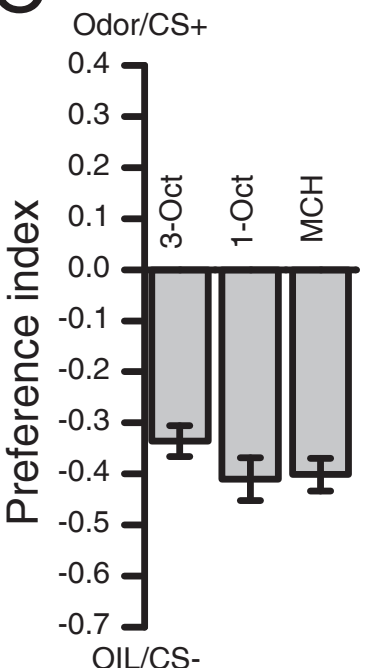

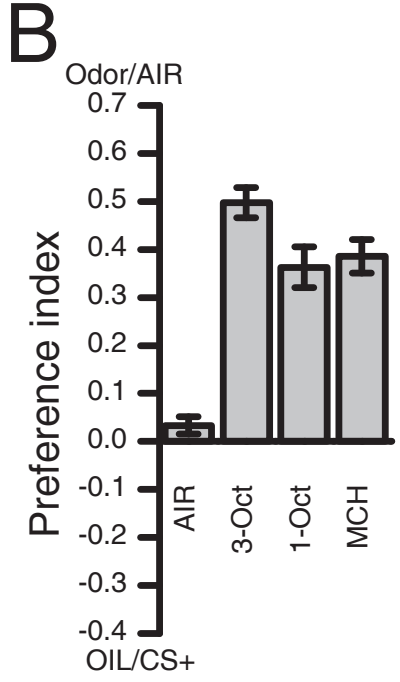

D

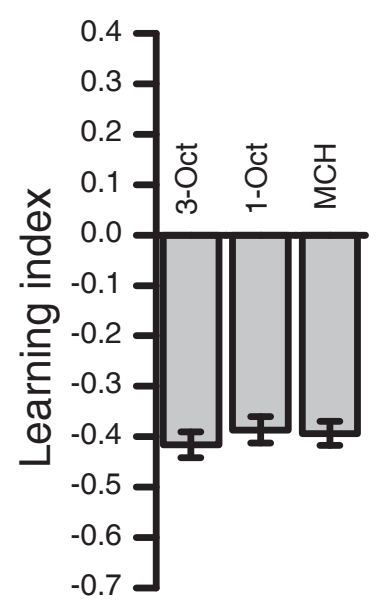

Figure 3.6. Final odor dilution determined by perexperiments.

Odorant dilutions were chosen regarding the untrained response of the flies in a T-maze test and the learnability (see figures 3.4 \& 3.5). Approx. 100 flies are placed in the training tube and exposed to an odorant and subsequently subjected to the solvent (mineral oil). Afterwards, the flies are transferred in a T-maze and can choose to approach or avoid the odor. (A) Untrained flies approached all three odorants at the given dilutions equally and show no preference when given the choice between mineral oil and an empty odor cup (air). (B) If the presentation of mineral oil is paired with an electric shock, the preference towards the odorants and an empty odor cup slightly increases. (C) Upon presentation of the odorants paired with electric shocks, the flies avoid all three odorants with a simi-

lar preference index. (D) Reciprocal training of the three odorants against mineral oil does not show significant differences between the learnability of the odorants. All three odorants can be learned equally well as reciprocal training against mineral oil leads to similar learning indices. 


\section{Results}

an odorant or the solvent with an electric shock can be combined to result in a reciprocal experiment in which the learnability of each of the three odorants against mineral oil does not differ from each other (Figure 3.6 D).

As a result of these experiments, odorant dilutions that do not have different properties in the innate behavior and the learnability of the flies could be found. Thus, the prerequisite to investigate generalization effects of these odorants is fulfilled. In a subsequent set of experiments, the generalization of the two structurally similar odorants 1-Oct and 3-Oct was analyzed.

\subsection{Generalization learning of similar odorants}

During the first set of experiments, it was investigated on a behavioral level whether the structurally similar odorants 1-octen-3-ol (1-Oct) and 3-octanol (3-Oct) are also behaviorally perceived as similar by the flies. Therefore, it was observed whether the flies generalize between the two odorants. If the flies in fact do generalize between two odorants, they show an equal or similar behavior in response to the odorants. The behavior will change with decreasing generalization and increasing discrimination. In particular, association of one odorant leads to an avoidance of a generalized odorant whereas a non-generalized odorant is not avoided and remains neutral. In order to test for generalization of the odorants, each odorant was trained reciprocally against the solvent (mineral oil). During the subsequent T-maze test, the flies had to decide to approach either the solvent or one of the odorants. It could be shown that wild type flies generalize between the odorants 1-octen-3-ol and 3-octanol as depicted in Figure 3.7. Avoidance of the generalized odorant can be observed, even though the learning index of the generalized odorant is significantly lower than the one from the trained odorant. A very weak generalization effect between $\mathrm{MCH}$ and 1-Oct (3-Oct respectively) was prominent after pairing of 1-Oct (3-Oct resp.) with electric shocks (Figure 3.7 A and B). However, no generalization could be observed after the association of $\mathrm{MCH}$ with electric shocks (Figure 3.7 C).

In conclusion, it could be observed that the two structurally similar odorants are also perceived very similar on a behavioral level. However, the generalization of the odorants was not complete as the preference indices of the generalized odorant were 


\section{Results}

significantly different from the responses towards the originally trained odorant. As a consequence, it can be assumed that the two odorants can be distinguished if a discrimination of the two stimuli is required. In order to confirm this assumption and to investigate the activation patterns of the odorants in the antennal lobe, in vivo calcium imaging of olfactory sensory neurons was performed.
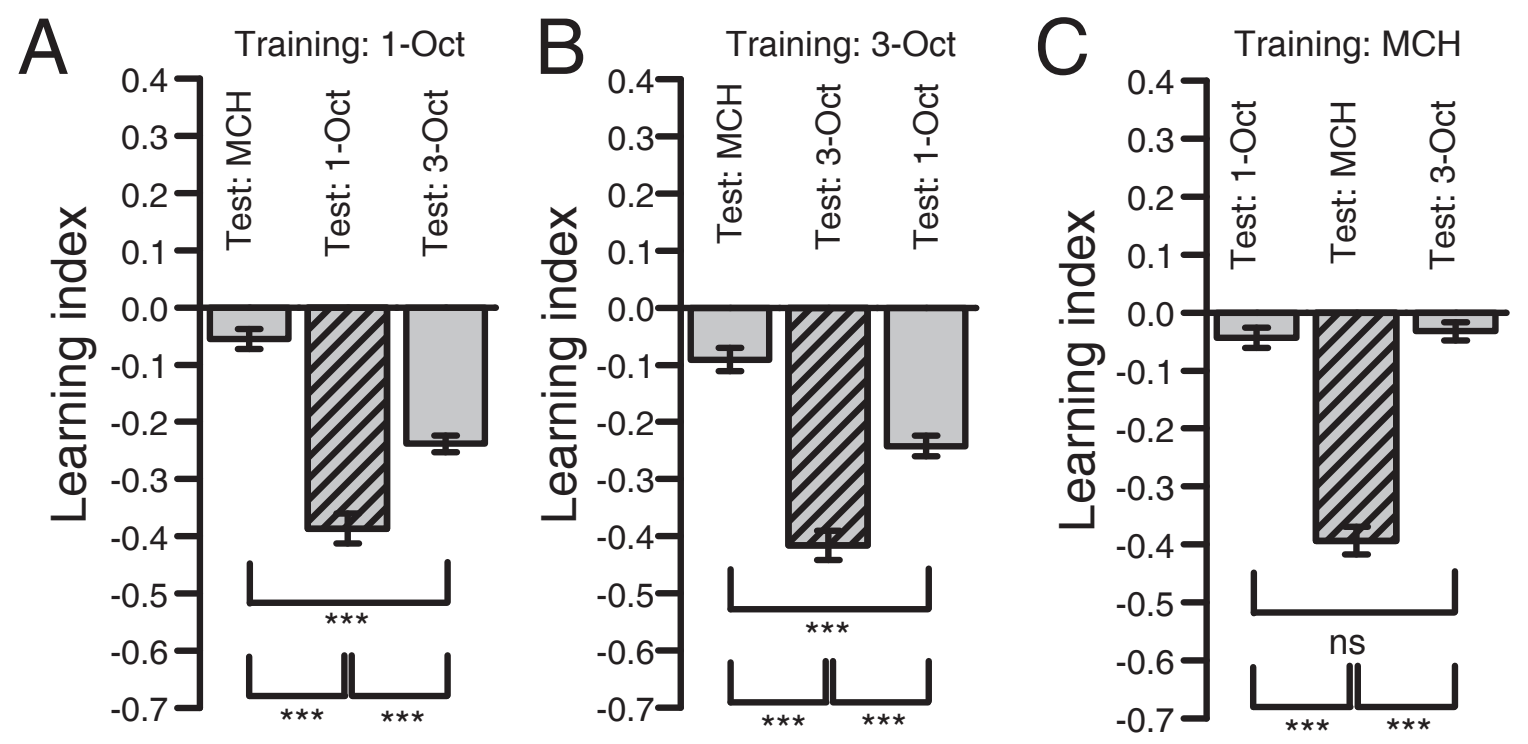

Figure 3.7. The chemically similar odorants are generalized.

Approx. 100 flies are placed in the training tube and exposed to an odor. During the application of the odor, the flies receive a series of electric shocks. Subsequent presentation of the solvent (mineral oil) is not paired with electric shocks. Afterwards, the flies are transferred in a T-maze and can choose to approach or avoid the odor. A reciprocal experiment, where the solvent is paired with the electric shock and not the odor, is used to calculate a learning index (mean of the reciprocal experiments). In each of the panels the trained odorant is displayed in the striped bar. (A) After reciprocal training of 1-Oct, the flies avoided 1-Oct and 3-Oct (even though significantly less than 1-Oct). An avoidance of $\mathrm{MCH}$ could also be observed but the learning index was comparably low. (B) Reciprocal training of 3-Oct resulted in an avoidance of 3-Oct and 1-Oct. $\mathrm{MCH}$ was much less avoided. (C) Reciprocal training of $\mathrm{MCH}$ resulted in an avoidance of only $\mathrm{MCH}$. 1-Oct and 3-Oct were not avoided and did not differ from each other.

$\mathrm{n}=16$ each; mean \pm SEM

ANOVA with post hoc Bonferroni corrected t-test; ${ }^{* \star *}=\mathrm{p}<0.001$ 


\section{Results}

\section{5. $\mathrm{Ca}^{2+}$-imaging of the three used odorants in the antennal lobe}

The structural similarity of 1-Oct and 3-Oct was confirmed on a behavioral level as a strong generalization could be observed. To investigate whether this similarity can also be found on a physiological level, the neuronal activation evoked by the three odorants used during the behavioral experiments (1-Oct, 3-Oct and $\mathrm{MCH}$ ) was measured with $\mathrm{Ca}^{2+}$-imaging in the antennal lobe. By the means of calcium indicators, the intracellular $\mathrm{Ca}^{2+}$-concentrations can be monitored. The changes in $\mathrm{Ca}^{2+}$-dynamics are a measure for neuronal activity as voltage depended $\mathrm{Ca}^{2+}$-channels are opening upon depolarization of the neurons [Stallcup, 1979;

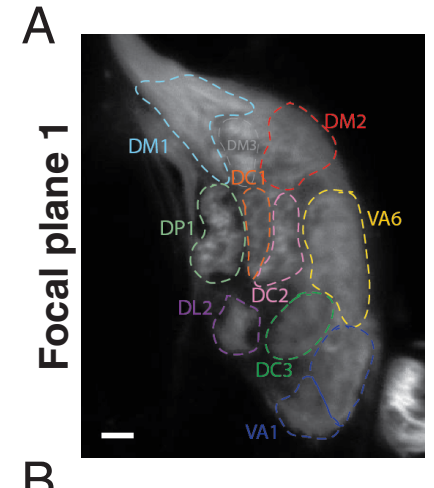

B

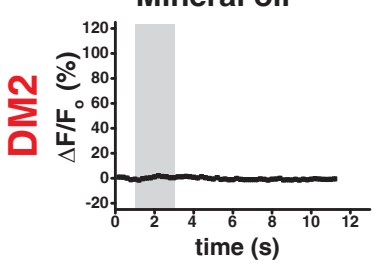

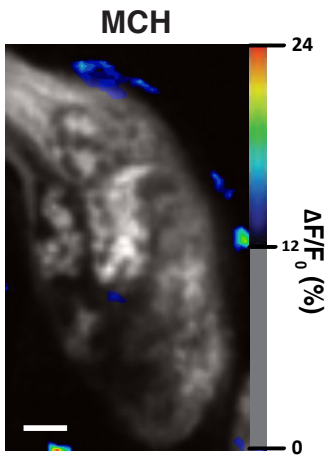

$\mathrm{MCH}$

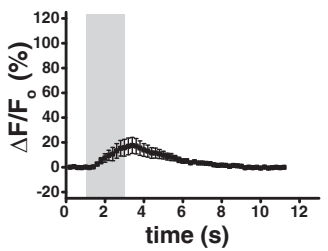

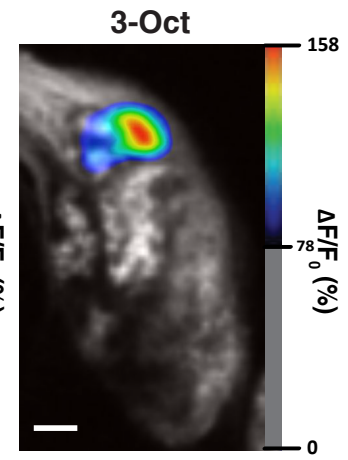

3-Oct

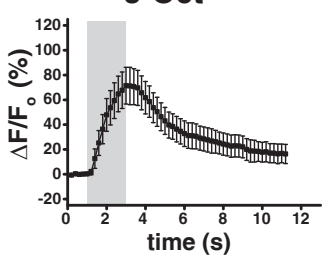

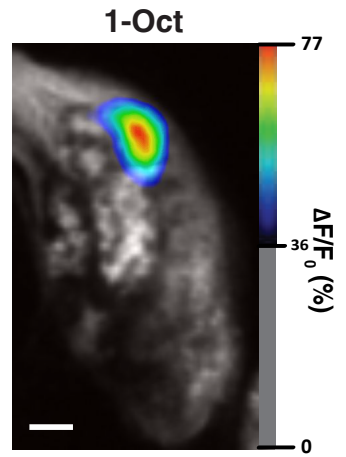

$1-$-Oct

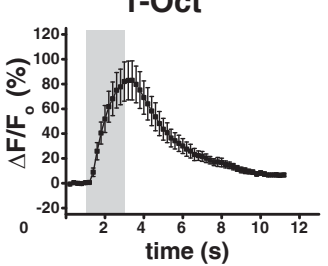

Figure 3.8. $\mathrm{Ca}^{2+}$-dynamics measured in the antennal lobe - focal plane 1.

(A) Example image of the first focal plane with outlines of identified glomeruli. Increased calcium activity in response to odorant application is depicted in false colors, superimposed on the image taken before stimulus onset. Signals lower than $50 \%$ of the maximum signal are cut off. In this focal plane, no response to $\mathrm{MCH}$ can be observed. The DM2 glomerulus responds to 3-Oct and 1-Oct. (B) Average intensity changes measured in 7 flies over time in the DM2 glomerulus ( \pm SEM). A weak response to $\mathrm{MCH}$ can be observed, whereas the intensity increase during 1-Oct and 3-Oct application is more prominent.

Scale bar: $10 \mu \mathrm{m}$ 


\section{Results}

Miyawaki et al., 1997; Berridge, 1998]. The spatial patterns of neuronal activity elicited by an odor are describing the odor representations within the structure of interest [Fiala et al., 2002; Griesbeck, 2004; Knöpfel et al., 2006].

The Or83b-Gal4 driver line [Wang et al., 2003a; Larsson et al., 2004] was used to express the genetically encoded calcium sensor GCaMP3.0 (under UAS control [Tian et al., 2009]) specifically in olfactory sensory neurons. Each of the odorants was presented in the same dilutions as during the behavioral experiments and transported with an air stream directly to the antennae of the fly. Intensity changes of the fluorescence reflect an increase in calcium concentration during the application of an odorant and are therefore a measure of neuronal activity [Guerrero \& Isacoff, 2001; Griesbeck, 2004]. Intensity changes were normalized to the baseline response and plotted over time for selected glomeruli. The glomeruli were identified with the help of optical slices and a 3D reconstruction of the antennal lobe [Laissue et al., 1999] available at http://www.flybrain.org [Armstrong et al., 1995]. Odorant-evoked changes in $\mathrm{Ca}^{2+}$-concentrations in the antennal lobe were imaged in two different focal planes in order to get a signal from all three odorants and a measurement for odor similarity. The false-color coded images shown in figures 3.8 and 3.9 visualize areas with an intensity increase of at least $50 \%$ of the maximum signal.

The first focal plane is located in the dorsal part of the antennal lobe and includes the DM2 glomerulus innervated by Or22a and Or22b neurons [Couto et al., 2005]. DM2 responded with a strong increase in the intracellular calcium concentration to the application of 3-Oct and 1-Oct but showed no signal towards MCH (Figure 3.8 A). Besides the response in DM2, no odorant evoked responses to $\mathrm{MCH}, 1-O c t$ and 3-Oct could be observed in this focal plane. In figure $3.8 \mathrm{~B}$ the change of fluorescence intensity and therefore the increase in calcium concentration as a measure of neuronal activity over time is depicted. Whereas the solvent mineral oil did not elicit any response, the glomerulus responded with a weak intensity increase towards $\mathrm{MCH}$. However, the responses during 1-Oct and 3-Oct application were considerably higher. This result is in line with previous studies about olfactory receptor and their ligands including Or22 and 3-Oct and/or 1-Oct [Galizia et al., 2010; Hallem et al., 2004; Fishilevich \& Vosshall, 2005; Hallem \& Carlson, 2006; Schmuker \& Schneider, 2007]. 


\section{Results}

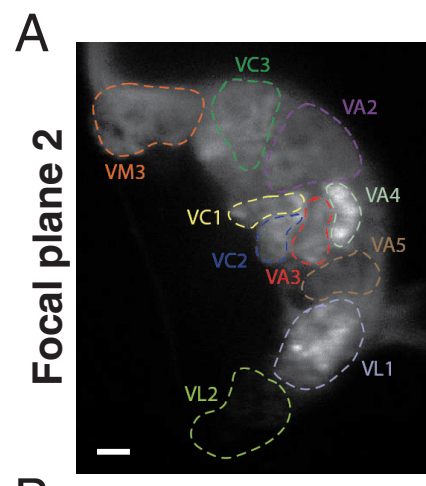

B

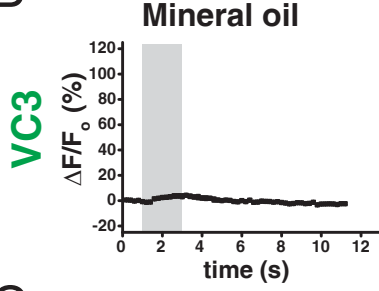

C

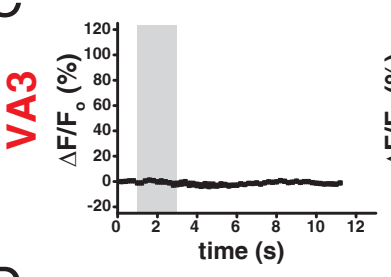

D

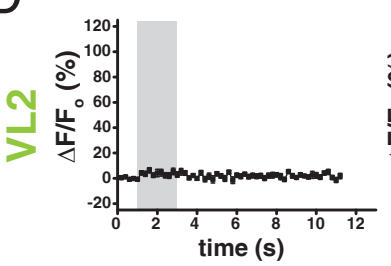

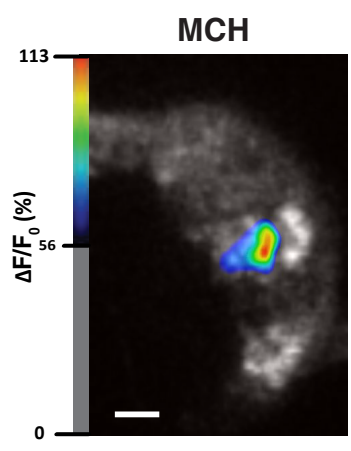

$\mathrm{MCH}$
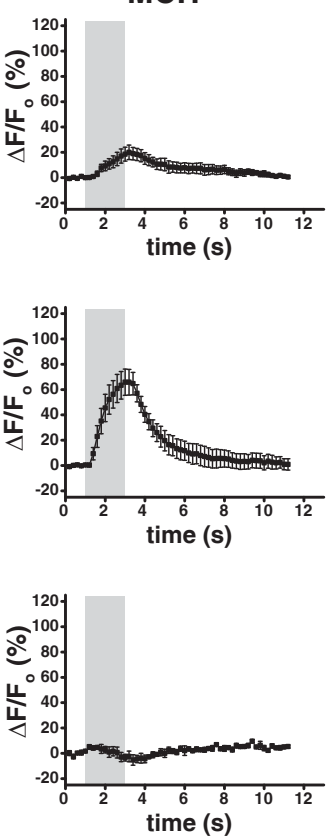

3-Oct

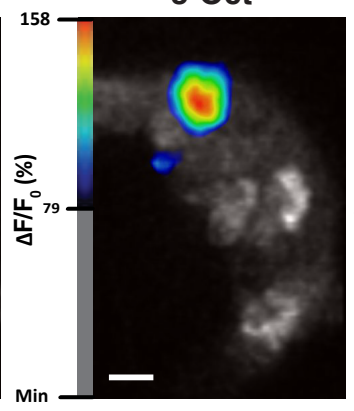

3-Oct
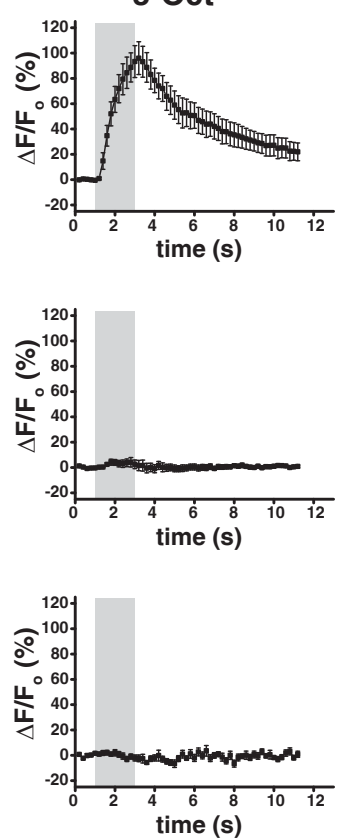

$1-$ Oct

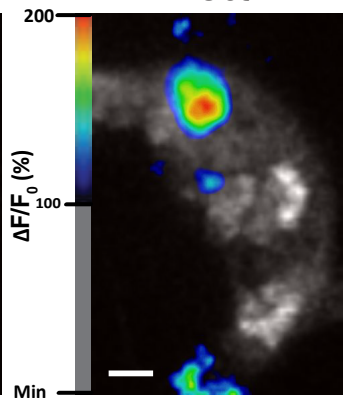

1-Oct
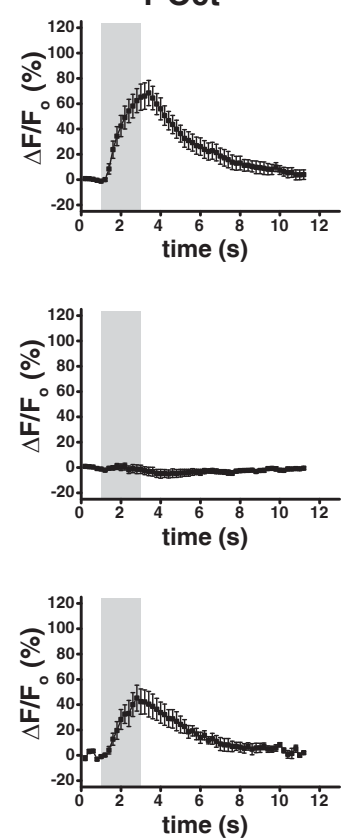

Figure 3.9. $\mathrm{Ca}^{2+}$-dynamics measured in the antennal lobe - focal plane 2.

(A) Outlines of identified glomeruli on an example image of the second focal plane. Superimposed on the image taken before the stimulus are false-color coded increased calcium concentrations in response to odorant applications. Signals lower than $50 \%$ of the maximum signal are cut off. (B-D) Average responses of 7 flies upon presentation of the three odorants over time in the three glomeruli with an intensity change of more than $50 \%$ of the maximum signal ( \pm SEM). VC3 responded weakly to $\mathrm{MCH}$ and strongly to 1-Oct and 3-Oct. In contrast, only $\mathrm{MCH}$ elicited a response in VA3. In VL2 only the presentation of 1-Oct resulted in an increase of fluorescence, whereas no response could be observed towards $3-\mathrm{Oct}$ and $\mathrm{MCH}$.

Scale bar: $10 \mu \mathrm{m}$ 


\section{Results}

The second focal plane is located at the ventral side of the antennal lobe and includes the VC3, VA3 and VL2 glomeruli (Figure 3.9 A). VC3 is innervated by neurons expressing Or35a, whereas VA3 consists of Or67b positive neurons [Couto et al. 2005). The olfactory receptor expressed by cells innervating VL2 could not be identified, even though a response to alcohols is described [Rodrigues, 1988] and an innervation by antennal coeloconic receptors is proposed [Couto et al., 2005]. VC3 responded weakly to a presentation of $\mathrm{MCH}$ and strong to 1-Oct and 3-Oct. A response to the solvent could not be observed (Figure 3.9 B). VA3 on the other hand responded only to $\mathrm{MCH}$ and did not show any change in the intracellular calcium concentration in response to 1-Oct and 3-Oct presentation (Figure 3.9 C). The responses obtained in VC3 and VA3 are comparable with previous results [Galizia et al. 2010). VL2 is almost out of focus and hardly visible in figure 3.9 A, but elicited a clear response towards 1-Oct. As the receptor type of VL2 innervating neurons is not known, a comparison with previous results was not possible. However, the responsiveness towards alcohols described by Rodrigues [1988] is supported by the signal obtained in response to 1-Oct.

Conclusively, the investigation of the response profiles of the three used odorants 1-octen-3-ol (1-Oct), 3-octanol (3-Oct) and 4-methylcyclohexanol (MCH) confirms a similarity of 1-Oct and 3-Oct also on a physiological level in the antennal lobes. The activation patterns evoked by $\mathrm{MCH}$ are more distinct. Hence, the structural similarity of 1-Oct and 3-Oct is not only confirmed by a similar perception manifested in a generalization behavior towards the odorants (section 3.4), but also by a physiological similarity in neuronal activity in the antennal lobes. However, the physiological responses towards the two odorants were not completely identical. As shown in figure 3.9, the VL2 glomerulus responded only to a presentation of 1-Oct and not to 3-Oct. As this confirms the assumption that the two odorants are not perceived the same, it was investigated if the flies could be trained to discriminate between the two similar odorants. 


\section{Results}

\subsection{Discrimination learning of similar odorants}

The results of the generalization experiments raised the question whether the two similar odorants 1-Oct and 3-Oct could be trained to be discriminated. Therefore, one of the odorants was associated with electric shocks whereas the other was presented without reinforcement. The outcome of this experiment led to the conclusion that the two odorants can be learned to be distinguished if they are trained differentially (Figure $3.10 \mathrm{~A}$ ). If one of the odorants was punished, the flies approached the other odorant and avoided the punished one. However, the learnability of 1-Oct versus 3-Oct was significantly lower than the learnability of each of the odorants versus the control odorant $\mathrm{MCH}$ (Figure 3.10 B). The structural similarity of 3-Oct and 1-Oct, which also results in a similar behavioral response and a comparable physiological
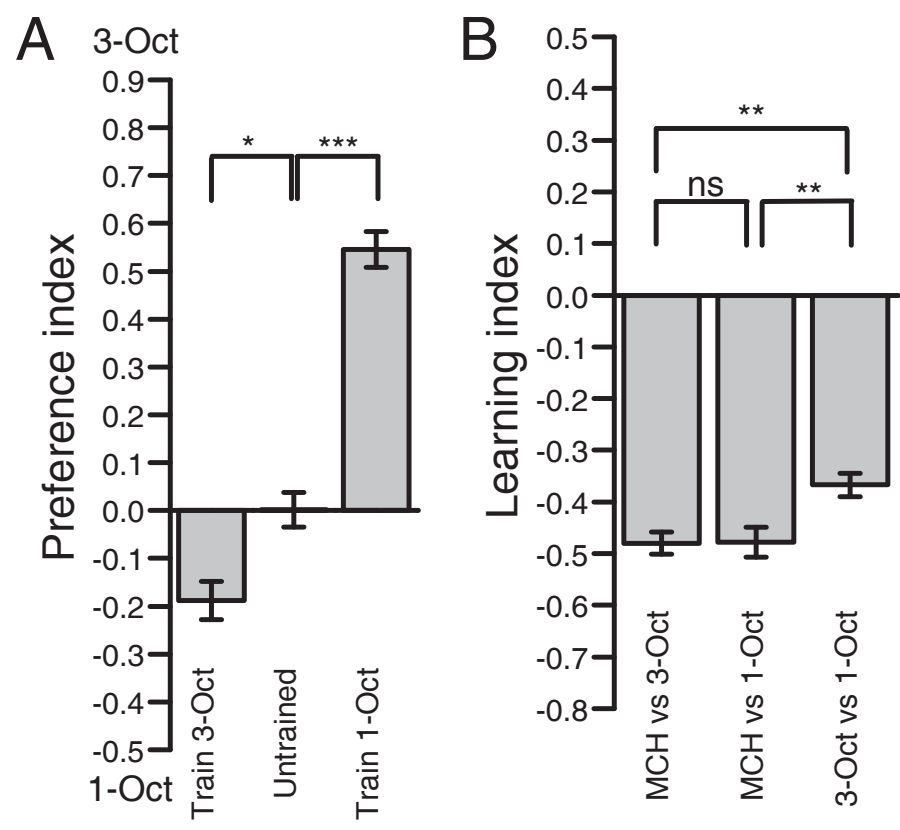

Figure 3.10. Discrimination learning of similar odorants. (A) 1-Oct and 3-Oct were trained differentially. Approx. 100 flies were placed in the training tube and the presentation of one odorant was paired with electric shock application (CS+). Subsequent presentation of the second odorant was not paired with electric shocks (CS-). In the T-maze situation, the flies could choose between the two odorants presented during the training. The flies avoided the odorant that was paired with the punishment. If none of the odorants was paired with electric shocks, the flies distributed equally in the

T-maze. (B) Even though reciprocal training of 1-Oct and 3-Oct could be distinguished after learning, the learning index that was significantly lower than the learning indices reached with the reciprocal training of $\mathrm{MCH}$ against 1 -Oct or 3-Oct.

$\mathrm{n}=16$ each; mean \pm SEM ANOVA with post hoc Bonferroni corrected t-test; ${ }^{*}=p>0.05 ;{ }^{* \star}=p<0.01 ;{ }^{* \star *}=p<0.001$ 


\section{Results}

response towards the odorants, was probably the reason for the increased difficulty to differentiate between them. The control odorant $\mathrm{MCH}$ is structurally distinct and also perceived as different, thus it is easier for the fly to discriminate between $\mathrm{MCH}$ and 3-Oct (1-Oct resp.). The similarity between 3-Oct and 1-Oct in contrast to $\mathrm{MCH}$, which could be observed in the behavior of the flies, was confirmed by visualizing the neuronal activation patterns of the three odorants with calcium imaging (see section 3.5). It has also to be mentioned that the punishment of 1-Oct led to a stronger avoidance of the odorant than the pairing of 3-Oct with the electric shock. This asymmetry might be explained by the different activation pattern of the two similar odorants in the antennal lobe. As visualized via the increase of the intracellular calcium concentrations, the activity pattern of 1-Oct and 3-Oct differed in a way that 3-Oct activated the same glomeruli as 1-Oct. However, additional glomeruli showed an increase of fluorescence during the presentation of 1-Oct. In previous studies, this pattern of additional activation by 1-Oct was also observed [Fishilevich \& Vosshall, 2005]. It can therefore be argued that it is easier for the fly to associate 1-Oct with the electric shock and 3-Oct with the absence of the punishment as the other way around. The possible influences of the effects of the reinforced (CS+) and the non-reinforced odorant (CS-) will be investigated later. Another important aspect of the investigation of odor similarity at this point is the neuronal foundation for the learned discrimination of the two similar odorants on the level of the antennal lobe.

\subsection{Blocking of synaptic transmission in inhibitory local interneurons of the antennal lobe}

A next step was to elucidate possible neuronal foundations for the generalization of 1 -Oct and 3-Oct and the possibility to discriminate them when it is required. Therefore, it was investigated if the function of inhibitory local interneurons in the antennal lobe was necessary for generalization and learned discrimination.

Silencing of different subpopulations of local interneurons was achieved by the use of transgenic flies. The shibire fly strain contains a defective gene for a protein necessary for endocytosis, called dynamin. Synaptic transmission is therefore blocked in shibire flies as the vesicle pool for exocytosis is depleted without ongoing 


\section{Results}

endocytosis [Kosaka \& Ikeda, 1983; van der Bliek \& Meyerowitz, 1991; Chen et al., 1991]. A temperature sensitive version of shibire $\left(\mathrm{shi}^{\mathrm{ts}}\right)$ expresses the mutant gene at a restrictive temperature of $32{ }^{\circ} \mathrm{C}$ whereas the wild type gene is expressed at $25^{\circ} \mathrm{C}$. The mutant allele of the shibire gene is dominant over the wild type [Kim $\&$ Wu, 1990). Therefore, synaptic transmission can be blocked by increasing the temperature [Kitamoto, 2001]. In order to find out more about the function of different subtypes of inhibitory local interneurons, two distinct classes of interneurons were assessed with different GAL4-enhancer-trap fly lines: NP2426-GAL4 and NP1227-GAL4. Type I inhibitory local interneurons (LN1) are targeted by NP1227 whereas NP2426 is expressing GAL4 in type II inhibitory local interneurons (LN2) [Sachse et al., 2007; Okada et al., 2009; Seki et al., 2010].

\subsubsection{Anatomy of two populations of local interneurons labelled by NP1227- and NP2426-GAL4}

A first step in the investigation of the inhibitory local interneurons included an anatomical analysis of the distribution of neurons targeted by the two GAL4enhancer-trap lines. Therefore, images were taken at a confocal microscope together with Dr. T. Riemensperger. Both lines were crossed with flies carrying two different GFP constructs under UAS control. The coupling of GFP with mCD8 results in a localization of GFP to the neuronal membranes whereas coupling with synaptobrevin targeted GFP to the synapses. Both constructs were expressed in the fly line $\left(w^{-} ; U A S-m C D 8: G F P, U A S-s y b: G F P ;+\right)$ used to visualize the cells targeted by NP1227-GAL4 and NP2426-GAL4 respectively. Using immunostainings, the signal of GFP was enhanced with a GFP antibody and brain neuropils were stained with the nc82-antibody against the presynaptic protein Bruchpilot [Hofbauer, 1991; Wagh et al., 2006]. Both types of interneurons innervate the whole antennal lobe and synapse within the glomeruli. However, the expression patterns differed substantially. 


\section{Results}

\section{Anatomy of NP1227-GAL4 positive neurons (type I local interneurons, LN1)}

The expression pattern of the NP1227 GAL4-enhancer-trap line targeting type I inhibitory local interneurons (LN1) shows a strong labeling of the antennal lobes in the central nervous system as previously described [Sachse et al., 2007]. Apart from the antennal lobe, only a few cells were expressing GFP in the fly brain. In the peripheral nervous system, the LN1 line labels a few glia cells around the thoracic ganglion, but no neuronal structures were stained with GFP (Figure 3.11A). A magnified view of the antennal lobe shows the labeled cell bodies of the inhibitory local interneurons in the surrounding of the lobe. Within the antennal lobe, the interneurons innervate a large amount of glomeruli. The neuronal arborizations are very distinct and sparsely target the core regions of the glomeruli (Figure $3.11 \mathrm{~B}$ ).
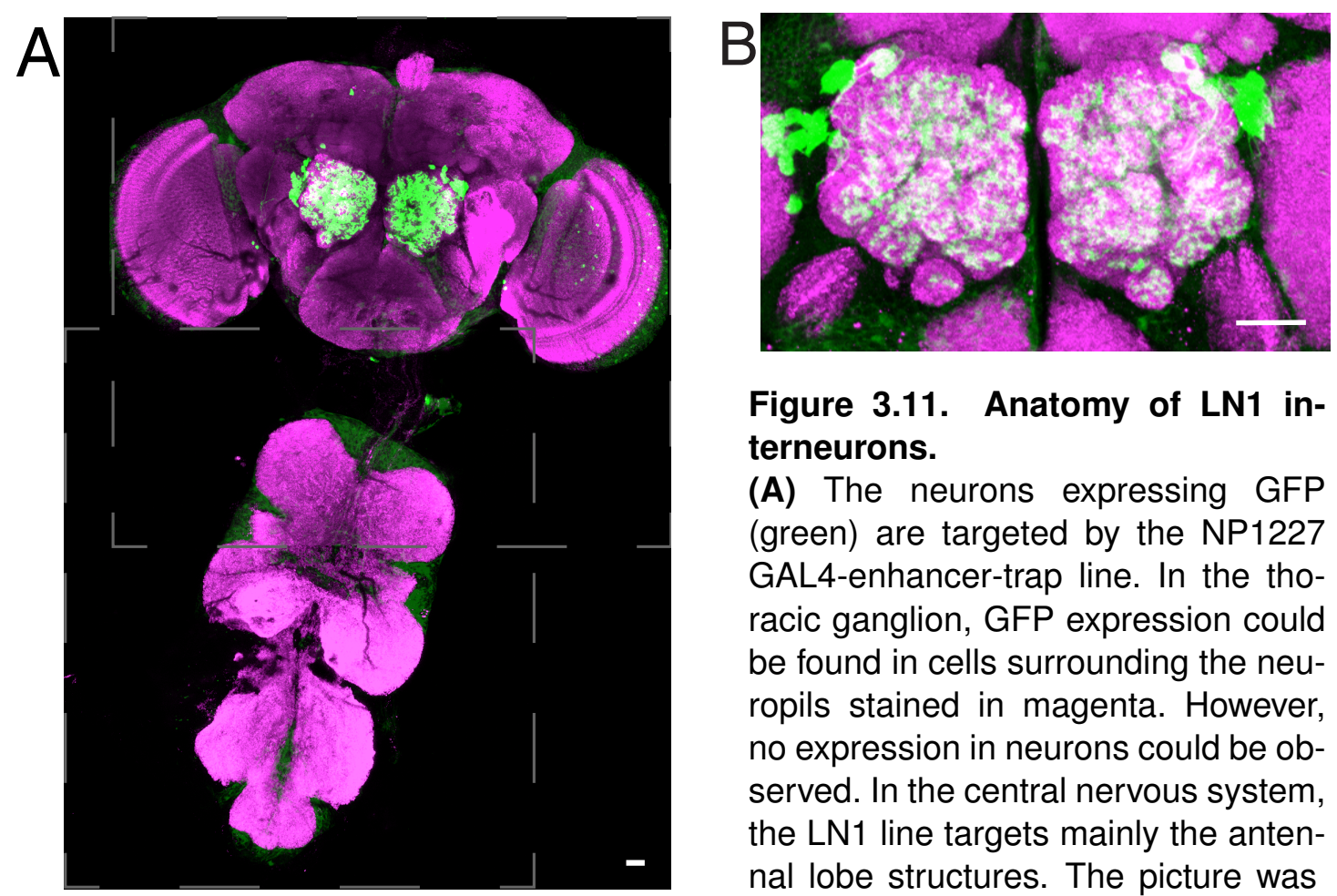

Figure 3.11. Anatomy of LN1 interneurons.

(A) The neurons expressing GFP (green) are targeted by the NP1227 GAL4-enhancer-trap line. In the thoracic ganglion, GFP expression could be found in cells surrounding the neuropils stained in magenta. However, no expression in neurons could be observed. In the central nervous system, the LN1 line targets mainly the antennal lobe structures. The picture was

merged from two separate scans in the same brain; gray dotted lines represent the borders of the two images. (B) A more detailed picture of the expression pattern within the antennal lobe reveals cell bodies surrounding the lobe structure. Neuronal processes extend inside the antennal lobe and arborize on the different glomeruli with distinct processes.

green: GFP; magenta: nc82; Scale bars: $25 \mu \mathrm{m}$ 


\section{Results}

\section{Anatomy of NP2426-GAL4 positive neurons (type II local interneurons, LN2)}

The NP2426 GAL4-enhancer-trap line targets type II inhibitory local interneurons. In the central nervous system, the GFP expression is mainly targeted to the antennal lobes as previously described [Sachse et al., 2007]. However, previous studies did not report the expression pattern of NP2426 in the peripheral nervous system. Here, the thoracic ganglion is also labeled by the NP2426 line (Figure 3.12 A). Several neuronal structures and cell bodies are targeted by NP2426 expression. In addition, a co-expression of GFP and the neuropil marker nc82 could be observed. The cell bodies labeled with GFP are most likely motorneurons that extend their arborizations into the legs [Baek \& Mann, 2009]. Expression of shibire in these neurons would block synaptic transmission and might therefore result in an impairment of locomotion.

The magnification of the antennal lobe shows a more detailed view of the neurons within the lobe that are targeted by NP2426-GAL4 (Figure 3.12 B). The cell bodies of the type II inhibitory local interneurons (LN2) are located outside of the antennal lobe and send their processes to the different glomeruli. As previously reported by Sachse et al. [2007], the innervation of the glomeruli is very dense in contrast to the type I inhibitory local interneurons (LN1). Moreover, the arborizations are targeting the core and the surface of the glomeruli and seem to be much thinner and more distinct than the ones from LN1 interneurons.

In conclusion, the targeting of both GAL4-enhancer-trap lines NP1227 and NP2426 to the antennal lobes could be confirmed. However, the labeling of motorneurons in the thoracic ganglion by the NP2426-line is a novel finding and might cause problems during olfactory conditioning with the Tully-machine. As a read-out during the test-phase of the paradigm includes the active avoidance or approach of one side of the T-maze, the line might not be suitable. Without normal locomotor activity, it is impossible to draw conclusion about the flies' behavior after absolute or differential training. Hence, several behavioral experiments were performed in order to investigate the general behavior of flies with a blocked synaptic transmission in both GAL4-enhancer-trap lines. 


\section{Results}
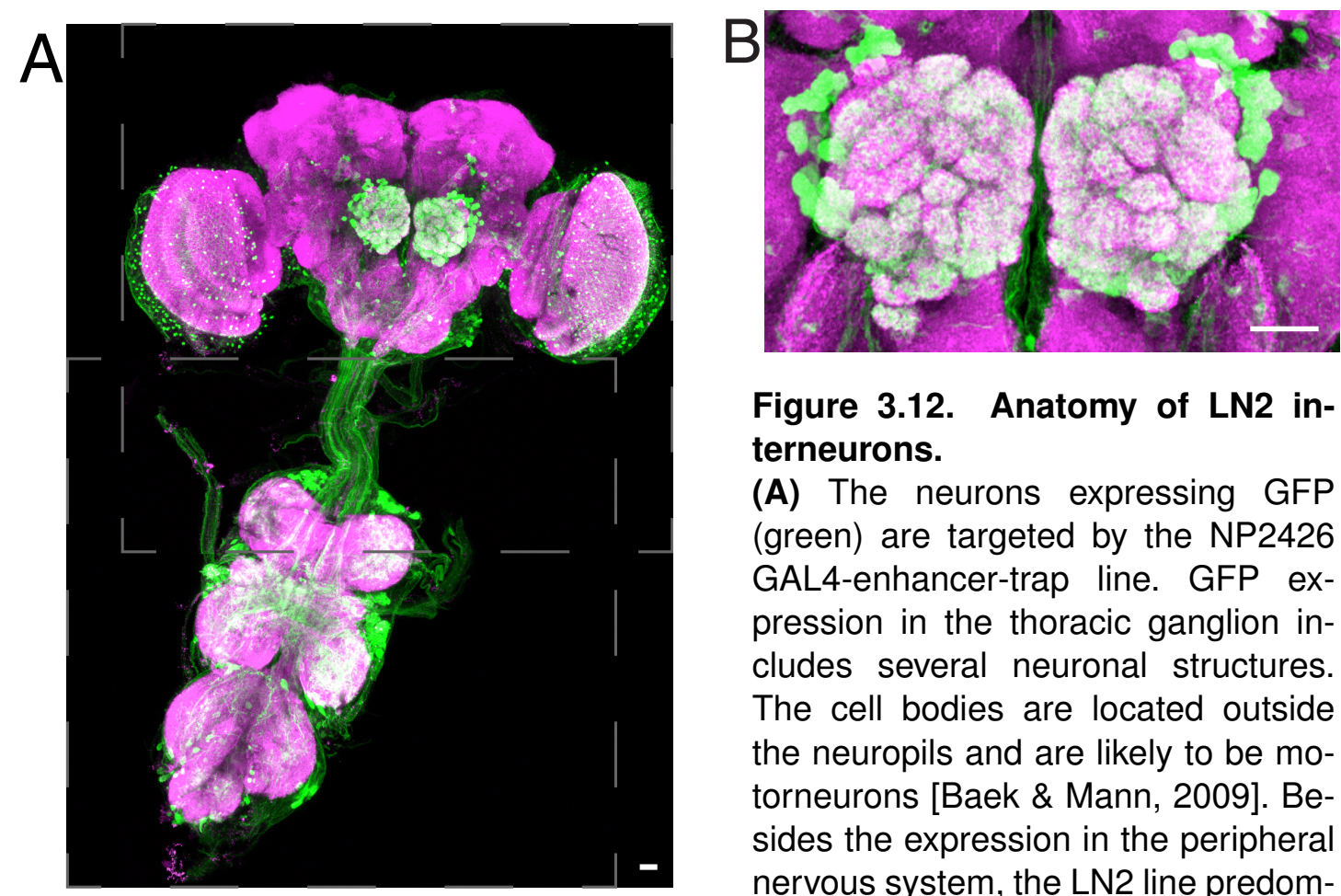

Figure 3.12. Anatomy of LN2 interneurons.

(A) The neurons expressing GFP (green) are targeted by the NP2426 GAL4-enhancer-trap line. GFP expression in the thoracic ganglion includes several neuronal structures. The cell bodies are located outside the neuropils and are likely to be motorneurons [Baek \& Mann, 2009]. Besides the expression in the peripheral nervous system, the LN2 line predominantly targets the antennal lobe in the central nervous system. The picture was merged from two separate scans in the same brain; gray dotted lines represent the borders of the two images. (B) A magnified view of the antennal lobes shows the cell bodies of local interneurons around the lobe structures. The neurons arborize within the lobe and broadly innervate the distinct glomeruli.

green: GFP; magenta: nc82; Scale bars: $25 \mu \mathrm{m}$

\subsubsection{General behavioral performance of flies with blocked synaptic transmission in two populations of local interneurons}

Anatomical studies of the GAL4-enhancer-trap lines NP1227 and NP2426 showed a distinct expression of GAL4 (reported by UAS-controlled GFP expression) in the antennal lobes. However, the additional targeting of NP2426 to motorneurons in the thoracic ganglion suggested an analysis of the general performance of the flies in different behavioral situations while the synaptic transmission is blocked with shibire 


\section{Results}

[Kitamoto, 2001]. The general locomotor activity of the flies was investigated using a climbing assay [Ganetzky \& Flanagan, 1978; Feany \& Bender, 2000; Friggi-Grelin et al., 2003]. Additionally, the behavior inside the Tully-machine was assayed by a shock avoidance test and the ability to smell 1-Oct and 3-Oct was assessed with an odor preference test.

\section{Climbing Assay}

The climbing assay is a way to measure the locomotor activity of flies. During the assay, the negative geotaxis of adult Drosophila is utilized. When tapped down to the bottom in a long cylinder, healthy flies are walking up towards the top. Flies expressing shibirets under the control of NP1227 (NP1227>Shits) do not show any difference in locomotor activity compared to the genetic controls at the permissive temperature of $25{ }^{\circ} \mathrm{C}$. Moreover, abolishing exocytosis and thereby blocking of synaptic transmission at an increased temperature of $32{ }^{\circ} \mathrm{C}$ did not result in a decrease in the climbing index (Figure $3.13 \mathrm{~A}$ ). Conclusively, a block of synaptic transmission in cells targeted by NP1227-GAL4 does not alter the locomotor activity of the flies.

A different situation could be observed in flies where the shibire $e^{\text {ts }}$ expression was driven by the NP2426 line. Already at the permissive temperature of $25{ }^{\circ} \mathrm{C}$ a difference between the test group (NP2426>Shits) and the heterozygous parental lines could be observed. NP2426>Shits showed an increased locomotor activity compared to the UAS-Shits control. However, the heterozygous NP2426 line also showed a slightly increased activity, which hints towards an effect of the genetic background as a reason for the increased activity of the NP2426 $>$ Shits flies. Nevertheless, this increase in locomotion was reduced when the temperature was set to $32{ }^{\circ} \mathrm{C}$. Whereas the UAS-Shits genetic control did not show any difference in behavior, the heterozygous NP2426 line showed a minor decrease already. Interestingly, the flies expressing shibire ${ }^{\text {ts }}$ under NP2426 control had a strongly reduced climbing index (Figure $3.13 \mathrm{~B}$ ).

The lower locomotor activity in the NP2426 parental line can only be explained by an effect of the genetic background. However, the decrease in the preference index 


\section{Results}

was not significant for the NP2426 control whereas NP2426>Shits even showed a significant decrease in locomotor activity towards the NP2426 line. This goes in line with the observation of GFP expression in motorneurons in the thoracic ganglion (see section 3.7.1). A synaptic silencing of motorneurons innervating the legs is a reasonable explanation for a decrease in locomotion.

In addition to the investigation of the general locomotor activity of flies with a silenced synaptic transmission in neurons targeted by NP1227 and NP2426, the avoidance of electric shocks in the Tully-machine was assessed. Additionally, the responses to odorant stimulation with 1-Oct and 3-Oct was tested.
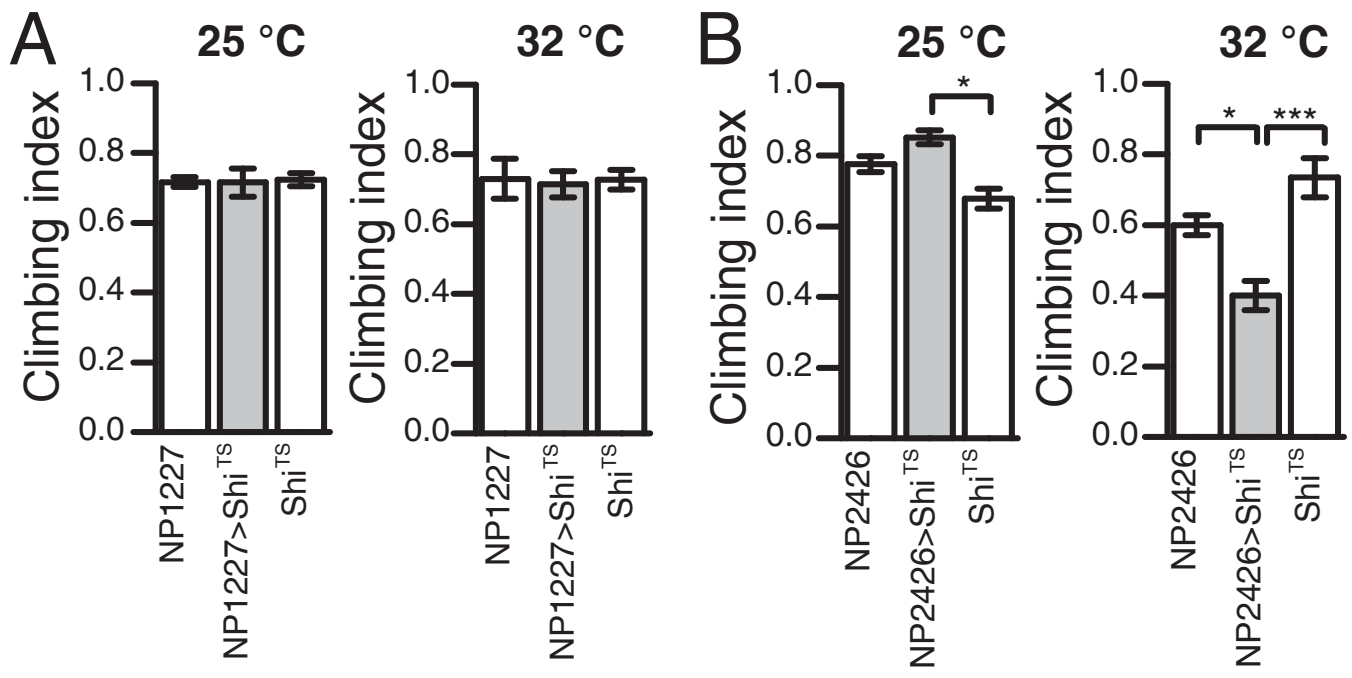

Figure 3.13. Locomotor performance of flies with blocked synaptic transmission in NP1227-GAL4 and NP2426-GAL4. Groups of 100 flies were subjected to the climbing assay. They were tapped to the bottom of a $28 \mathrm{~cm}$ long serological pipette and could subsequently climb up. After one minute, it was counted how many flies are in the upper, middle and lower compartment of the pipette and a climbing index calculated. (A) At the permissive temperature of $25^{\circ} \mathrm{C}$ as well as at the restrictive temperature of $32{ }^{\circ} \mathrm{C}$, the locomotor activity of flies with expression of shibire ${ }^{\text {ts }}$ in cells targeted by the NP1227 line was the same as in the genetic controls. (B) Flies expressing shibire ${ }^{\text {ts }}$ in cells targeted by NP2426 show an increased locomotor activity at the permissive temperature of $25^{\circ} \mathrm{C}$ compared to the genetic controls. At the restrictive temperature of $32{ }^{\circ} \mathrm{C}$, the locomotor activity of flies expressing shibire ${ }^{\text {ts }}$ under the control of NP2426 is significantly decreased.

$\mathrm{n}=5$ each; mean \pm SEM ANOVA with post hoc Bonferroni corrected t-test; ${ }^{*}=p<0.05 ;{ }^{* * *}=p<0.001$ 


\section{Results}

\section{Shock Avoidance}

One crucial prerequisite to study associative olfactory learning with the paradigm described by Tully \& Quinn [1985] is the ability of flies to perceive electric shocks and recognize the stimuli as a negative reinforcer. It is therefore essential that the flies show an avoidance of electric shocks. Hence, experiments were performed to investigate the behavior towards punishing electric shocks. The flies were placed in the elevator section of the Tully-machine for a test in the T-maze. One arm of the maze comprised a normal test tube whereas the other arm consisted of the training tube with 12 electric shocks of $90 \mathrm{~V}$ DC delivered over one minute (1.25 sec shock and $3.75 \mathrm{sec}$ inter pulse interval) during which the flies could decide for one arm of the T-maze.
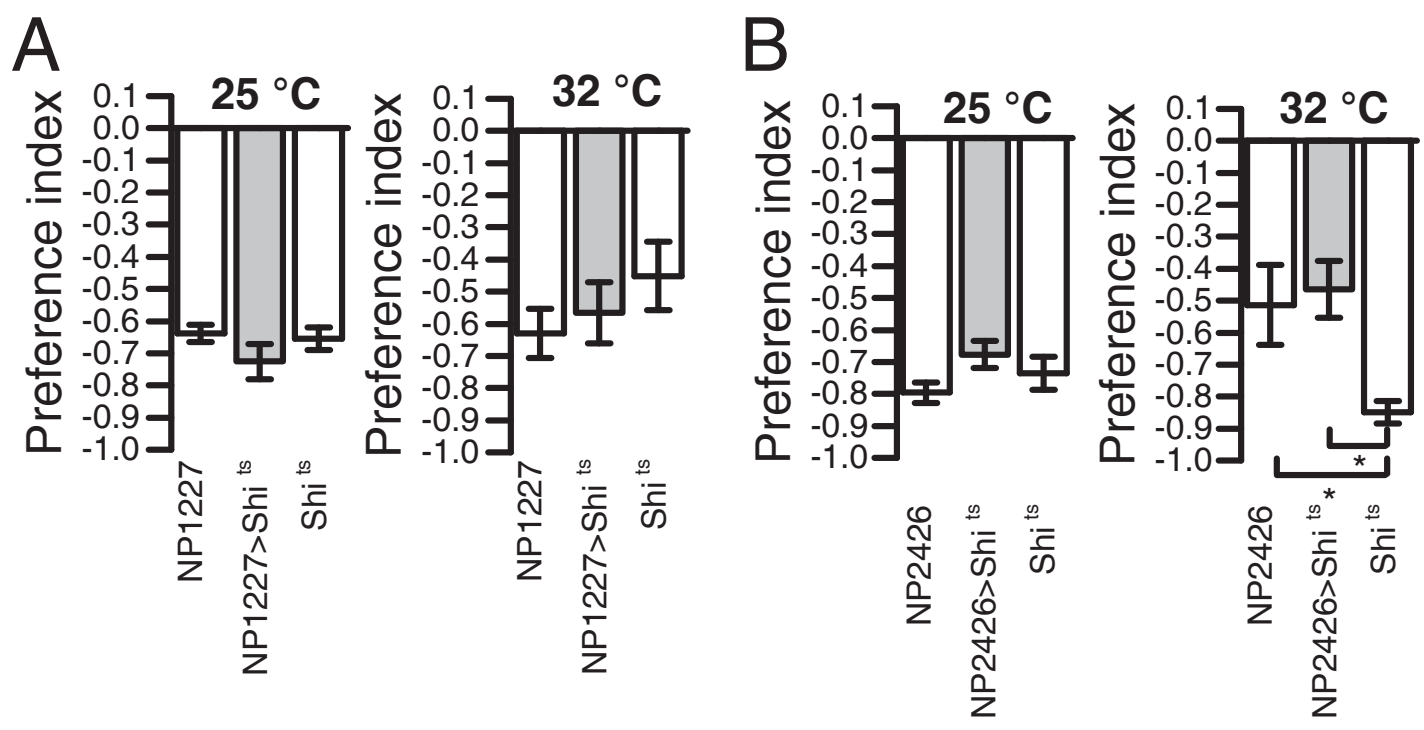

Figure 3.14. Shock avoidance of flies with silenced NP1227 and NP2426 neurons. Avoidance of the electrified training tube (90 V DC) in a T-maze was measured by placing $\sim 100$ flies in the machine. The other side of the maze consisted of a nonelectrified test tube. (A) Silencing of synaptic transmission in NP1227 neurons did not alter the shock avoidance of flies expressing shibire $e^{\text {ts }}$ in neurons targeted by NP1227. (B) At the permissive temperature of $25^{\circ} \mathrm{C}$, all tested lines showed a similarly strong shock avoidance. At $32{ }^{\circ} \mathrm{C}$, the shibire ${ }^{\text {ts }}$ parental line showed a normal shock avoidance whereas both the NP2426 parental line as well as the NP2426>Shits line showed a significant decrease in the preference index.

$\mathrm{n}=8$ each; mean \pm SEM

ANOVA with post hoc Bonferroni corrected t-test; ${ }^{*}=\mathrm{p}<0.05$ 


\section{Results}

The expression of shibire ${ }^{\text {ts }}$ under NP1227 control did not result in a different shock avoidance performance at the permissive temperature of $25^{\circ} \mathrm{C}$. The combination of NP1227-GAL4 and UAS-Shits showed a similar preference index when compared with the genetic controls. An increase of the temperature to $32{ }^{\circ} \mathrm{C}$ to block synaptic transmission did not change the preference index (Figure 3.14 A).

At $25{ }^{\circ} \mathrm{C}$, the heterozygous parental lines as well as NP2426>Shits preferred the arm of the T-maze without the electrified grid. However, at the restrictive temperature of $32{ }^{\circ} \mathrm{C}$, the avoidance of the electric shock was significantly decreased by Np2426>Shits and the NP2426 line (Figure 3.14B). This behavior can be explained with the decreased locomotor ability of flies expressing shibire under NP2426 control (see above). Interestingly, also the NP2426-GAL4 line had a decreased response towards the electric shock. When the reduced locomotor activity at $32{ }^{\circ} \mathrm{C}$ is taken into account, the genetic background might be an explanation. Even though these results already suggest to exclude NP2426 flies from further experiments, the olfactory behavior towards 1-Oct and 3-Oct was investigated for both GAL4-enhancer-trap lines combined with the UAS-Shits line and their genetic controls.

\section{Odor preference towards $1-$ Oct and 3-Oct}

Olfactory learning experiments require the ability to perceive the odorants that are going to be trained. In order to confirm the flies' ability to smell, odor preference experiments were performed with all fly lines that were to be used during synaptic blocking of inhibitory local interneurons: NP1227>Shits, NP2426>Shits and the genetic controls.

The behavioral responses of NP1227>Shits, NP1227-GAL4 and UAS-Shits flies towards 1 -Oct and 3-Oct were very similar between groups and comparable to the wild type (see also Figure 3.6) at the permissive temperature of $25^{\circ} \mathrm{C}$. In the case of 1-Oct, the preference index indicating the approach towards the odorant was nonsignificantly higher for NP1227>Shits and the NP1227-GAL4 genetic control compared to the UAS-Shits control. (Figure $3.15 \mathrm{~A}$ ). At $32{ }^{\circ} \mathrm{C}$ the appetitive behavior of all groups was decreased. However, the preference index was similar for all groups. The approach towards 3-Oct was not affected by a change in the temperature (Figure $3.15 \mathrm{C})$. 


\section{Results}

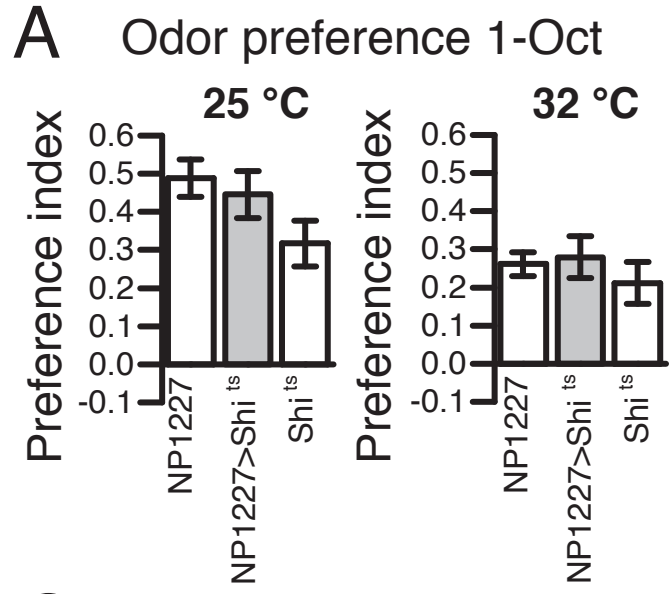

C Odor preference 3-Oct
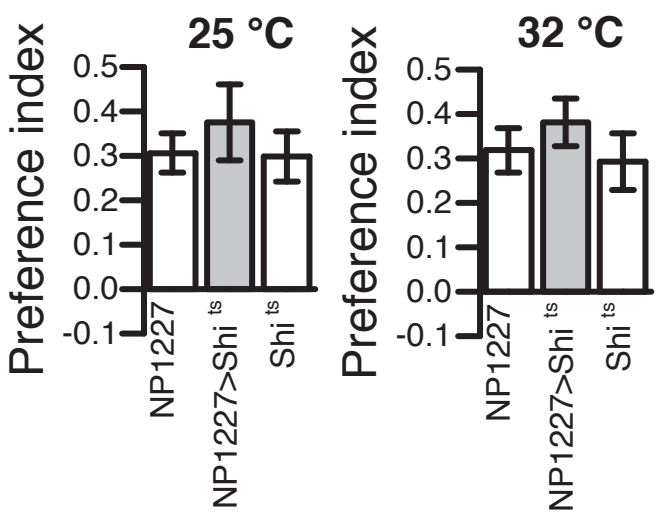

B Odor preference 1-Oct
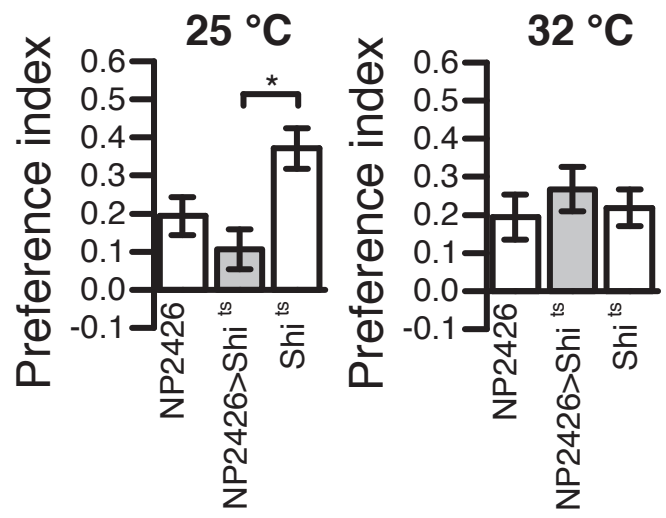

D Odor preference 3-Oct

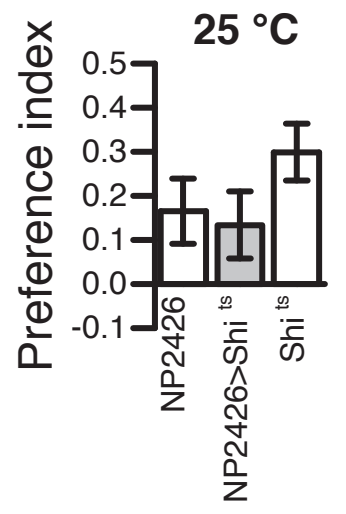

Figure 3.15. Odor preference of NP1227>Shits and NP2426>Shits.

Groups of $\sim 100$ flies were transferred into the Tully-machine and subjected to a T-maze test. On one side, either 1-Oct or 3-Oct was presented whereas the other side contained mineral oil. The flies were approaching one of the two sides and subsequently counted. $(\mathbf{A}+\mathbf{C})$ NP1227>Shits flies did not show a different behavior towards 1-Oct or 3-Oct compared to their genetic controls. However, a decrease in 1-Oct approach could be observed at $32{ }^{\circ} \mathrm{C}$ for NP1227>Shits and the NP1227 parental line. (B+D) At the permissive temperature of $25^{\circ} \mathrm{C}$, the NP2426>Shits and the NP2426 genetic control showed only a weak approach of either 1-Oct or 3-Oct. In the case of 1-Oct, the approach of NP2426>Shits was even significantly lower compared to the UAS-Shits genetic control. An increase of the temperature to $32{ }^{\circ} \mathrm{C}$ resulted in a decreased approach of 1-Oct for the UAS-Shits parental control, similar to the response of NP1227 and the genetic controls. However, the response of NP2426>Shits and NP2426 was not affected by the temperature change. $\mathrm{n}=8$ each; mean \pm SEM 


\section{Results}

The responses of NP2426>Shits and the NP2426-GAL4 heterozygous control (see crossing schemes in appendix $B$ towards 1-Oct and 3-Oct were smaller than the UAS-Shits heterozygous control. In the case of 1-Oct, this decrease was even significant. As the preference index of UAS-Shits was comparable to the index achieved by wild type flies and during the NP1227 experiments, an effect of the genetic background of NP2426-GAL4 can be assumed. A change of the temperature to $32{ }^{\circ} \mathrm{C}$ decreased the approach of the UAS-Shits control towards 1-Oct but did not alter the response of NP2426>Shits and NP2426-GAL4 (Figure 3.15 B). Likewise, the preference index of all three groups towards $3-\mathrm{Oct}$ at $32{ }^{\circ} \mathrm{C}$ was comparable to the responses at $25^{\circ} \mathrm{C}$ (Figure $\left.3.15 \mathrm{D}\right)$.

In conclusion, the results of the experiments investigating the general behavioral performance of flies with blocked synaptic transmission in cells targeted by NP1227GAL4 and NP2426-GAL4 suggested to continue only with NP1227-GAL4 during further learning experiments. Expression of shibire ${ }^{\text {ts }}$ under NP1227-GAL4 control did not alter the locomotor activity, shock avoidance and odor perception of the flies at the permissive temperature of $25{ }^{\circ} \mathrm{C}$. In the same way, the performance was not changed when the temperature was increased to $32{ }^{\circ} \mathrm{C}$ in order to activate the mutant form of dynamin and therefore block synaptic transmission. Unfortunately, this was not the case for the NP2426 flies.

Already the heterozygous NP2426-GAL4 parental control showed a difference in locomotor activity and shock avoidance at $32{ }^{\circ} \mathrm{C}$ (Figures $3.13 \mathrm{~B}$ and $3.14 \mathrm{~B}$ ). This difference in performance at $32{ }^{\circ} \mathrm{C}$ was also observable in NP2426>Shits and significantly increased in the climbing assay used to test locomotor activity. The anatomical investigation of NP2426-GAL4 expression suggested the targeting of motorneurons in the thoracic ganglion as a possible explanation for the significant decrease in locomotor activity of NP2426>Shits flies (Figure 3.12 A).

Additionally, the response towards 1-Oct and 3-Oct was different in NP2426>Shits and NP2426-GAL4 already at $25^{\circ} \mathrm{C}$ (Figure $3.15 \mathrm{~B}$ ). This alteration of the olfactory response without activation of shibire ${ }^{\text {ts }}$ was arguing against a use of NP2426 in further experiments. Additionally, the response of these lines was not changed after an increase of the temperature whereas the preference index of the UAS-Shits genetic control towards 1-Oct decreased comparable to the decrease observed in the NP1227 flies. This difference in the behavior of the test group and the genetic 


\section{Results}

controls strengthened the decision to exclude the NP2426 line from further experiments. As a conclusion from the test for behavioral performance of flies with blocked synaptic transmission in cells targeted by NP2426-GAL4 or NP1227-GAL4, further experiments were only performed with the NP1227 line that targets type I inhibitory local interneurons (LN1).

\subsubsection{Absolute learning}

Expression of shibire ${ }^{\text {ts }}$ in type I inhibitory local interneurons was accomplished with the NP1227-GAL4-enhancer-trap line [Sachse et al., 2007; Okada et al., 2009; Seki et al., 2010]. After reciprocal absolute training of 1-Oct against mineral oil, a stable learning could be observed. At the permissive temperature of $25^{\circ} \mathrm{C}$, without expres-

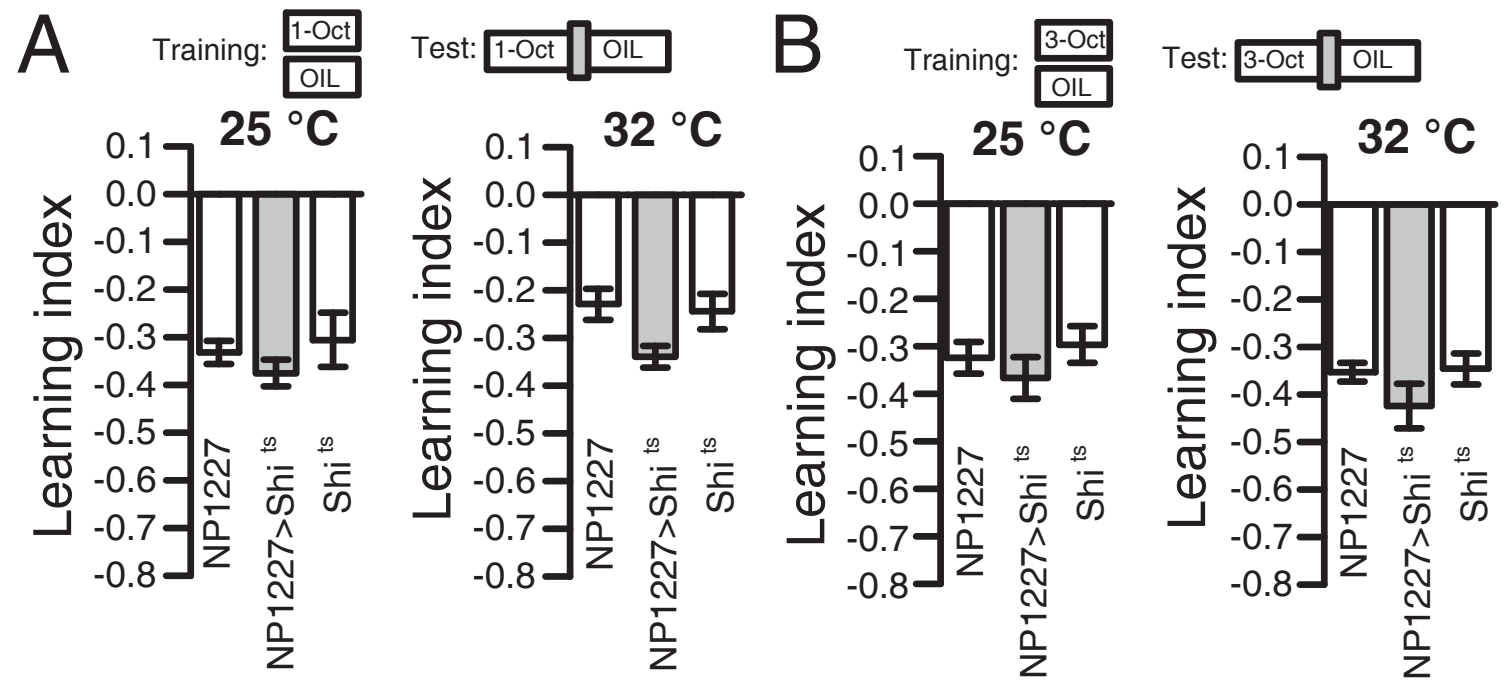

Figure 3.16. Absolute conditioning of NP1227 $>\mathrm{Shi}^{\text {ts }}$. Absolute training of 1-Oct or 3-Oct against mineral oil in reciprocal experiments with $\sim 100$ flies resulted in a learned avoidance. The learning indices did not differ in test and control groups both at the permissive and the restrictive temperature of shibire. (A) Association of 1-Oct with the electric shock led to an avoidance of the odorant at $25^{\circ} \mathrm{C}$. NP1227>Shits showed a slightly increased learning index. This higher learning index was even more prominent but not significant after the block of synaptic transmission at $32{ }^{\circ} \mathrm{C}$. (B) Reciprocal training of 3-Oct against mineral oil resulted in a similar learning index in the test and the control groups. At both temperatures, the test group (NP1227>Shits) showed a slightly increased learning. $\mathrm{n}=9-12$ each; mean \pm SEM 


\section{Results}

sion of the mutant form of dynamin and therefore intact synaptic transmission, the flies showed a similar learning index in all groups. The achieved learning scores were comparable to the indices of wild type flies (see figure 3.6). Interestingly, the test group (NP1227>Shits) showed a slightly increased learning index. This stronger learning could also be observed in flies trained and tested at the restrictive temperature of $32{ }^{\circ} \mathrm{C}$ and therefore blocked synaptic transmission in type I inhibitory local interneurons (LN1). It has to be mentioned that the difference between the test group and the genetic controls is larger at the higher temperature (however, not significant) due to a decrease in the learning index of the genetic controls. Interestingly, the reduced learning index for $1-$ Oct at $32{ }^{\circ} \mathrm{C}$ is comparable to the reduced innate attraction towards the odorant (see Figure $3.15 \mathrm{~A}$ ).

Absolute training of 3-Oct against mineral oil resulted in similar learning indices in all groups for the permissive and the restrictive temperature. As described for absolute training of 1-Oct before, the test group (Np1227>Shits) showed a slightly, but not significant, increased learning compared to the controls at both temperatures (Figure $3.15 \mathrm{~B})$.

Conclusively, the silencing of LN1 does not alter the ability of the flies to associate an odorant with an electric shock. As a next step, it was investigated whether a blocking of synaptic transmission in type I inhibitory local interneurons has an effect on generalization of similar odorants.

\subsubsection{Generalization learning of similar odorants}

In order to address the generalization of the two similar odorants 1-Oct and 3-Oct, experiments were performed in which one of the odorants was associated with an electric shock whereas the other one was presented during the test phase (comparable to the experiments in section 3.4 with wild type flies). Generalization between two odorants was assumed, when the avoidance of the non-reinforced odorant during the test situation was prominent.

All groups (the test group as well as the genetic controls) showed a generalization between 1-Oct and 3-Oct at the permissive and the restrictive temperature when 1-Oct was reinforced and 3-Oct was tested (Figure 3.17 A). Indeed, NP1227>Shits flies showed a slightly but not significantly stronger response than the genetic con- 


\section{Results}

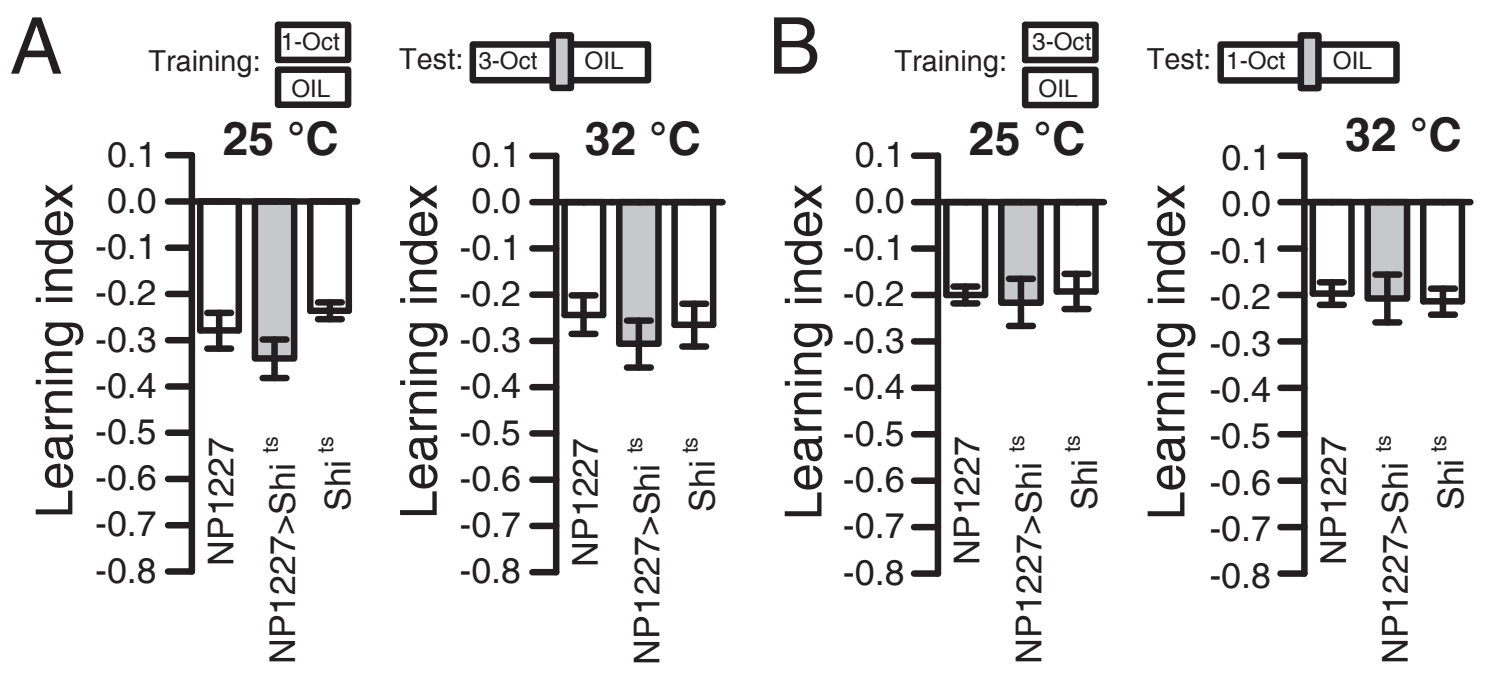

Figure 3.17. Generalization of 1-Oct and 3-Oct in NP1227 $>\mathrm{Shi}^{\text {ts }}$ flies.

Groups of approx. 100 NP1227>Shits flies and the genetic controls were trained absolutely against 1 -Oct or 3-Oct. During the subsequent test phase, the non-trained odorant was presented in one arm of the T-maze whereas mineral oil was presented in the other to test for the generalization of the two odorants. (A) When 1-Oct was trained and the response towards 3-Oct was tested, the behavior of the flies in all groups did not differ significantly at both temperatures. However, the NP1227>Shits flies showed a slightly increased generalization at 25 and $32{ }^{\circ} \mathrm{C}$. (B) In the case of 3-Oct training and subsequent test for 1-Oct avoidance, no difference between the groups and temperatures could be observed.

$\mathrm{n}=8$ each; mean \pm SEM

trols. When 3-Oct was reinforced and 1-Oct was presented during the test phase, the generalization was equally observable for all groups at 25 and $32{ }^{\circ} \mathrm{C}$. However, the achieved learning indices were weaker than after association of 1 -Oct with the shock.

In conclusion, it can be stated that the blocking of LN1 does not influence the generalization of 1-Oct and 3-Oct. Hence, the activity of type I inhibitory local interneurons seems not to be necessary for the flies' generalization of similar odorants. It was of subsequent interest, if the LN1 interneurons are necessary to discriminate between odorants. Therefore, a set of experiments was performed in which the two odorants were trained differentially. 


\section{Results}

\subsubsection{Discrimination learning of non-similar odorants}

During a first test for discrimination learning, two non-similar odorants (either 1-Oct or 3-Oct and the control odorant $\mathrm{MCH}$ ) were trained differentially: in a reciprocal experiment, one of the odorants was associated with an electric shock whereas the other was presented without reinforcement.

Discrimination learning of non-similar odorants was not affected by a block of synaptic transmission in type I inhibitory local interneurons. The learning index achieved after training of $1-\mathrm{Oct}$ and $\mathrm{MCH}$ at $25^{\circ} \mathrm{C}$ was similar to the learning index at $32{ }^{\circ} \mathrm{C}$ (Figure $3.18 \mathrm{~A}$ ). Likewise, the learning scores after differential training of 3-Oct and $\mathrm{MCH}$ were not altered by a difference in the temperature (Figure $3.18 \mathrm{~B}$ ). These results raised the question whether the discrimination learning of similar odorants might be affected by a silencing of synaptic transmission in LN1.
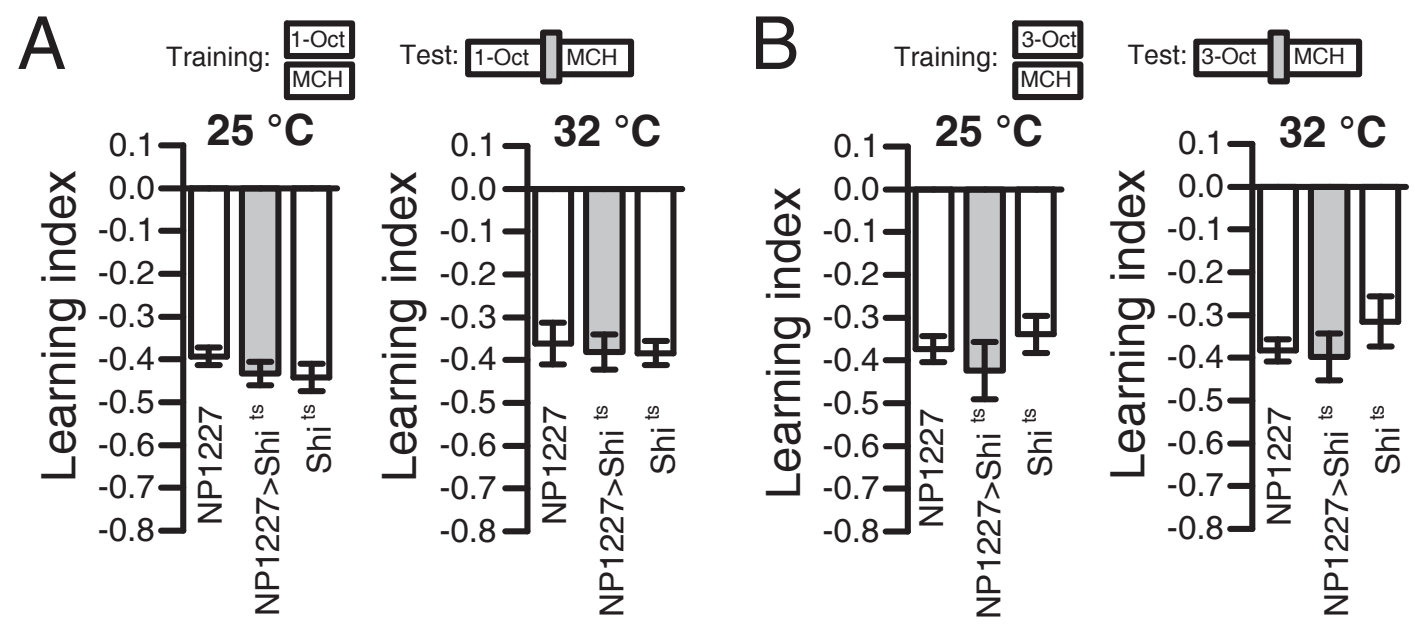

Figure 3.18. Discrimination of 1-Oct/3-Oct and $\mathrm{MCH}$ in NP1227>Shits flies.

NP1227>Shits flies and genetic controls ( 100 flies per experiment) were trained differentially with non-similar odorants. One of the odorants was temporailly paired with electric shocks (CS+) whereas the other was presented alone (CS-). Each odorant was used once as a CS+ and once as a CS- in reciprocal experiments. (A) Differential conditioning of 1-Oct and $\mathrm{MCH}$ in NP1227>Shits did neither show any difference in the learning index at $25^{\circ} \mathrm{C}$ nor at $32{ }^{\circ} \mathrm{C}$ when compared to the genetic controls. (B) 3-Oct versus $\mathrm{MCH}$ differential training resulted in similar learning indices for all groups at the permissive and the restrictive temperature. $\mathrm{n}=8$ each; mean \pm SEM 


\section{Results}

\subsubsection{Discrimination learning of similar odorants}

Differential learning of similar odorants resulted in a learned discrimination of innate generalized odorants in wild type flies (see section 3.6). In order to investigate a putative role of type I inhibitory local interneurons, synaptic transmission was blocked with shibirets in neurons targeted by NP1227. A reciprocal experiment was performed in which the presentation of one of the two
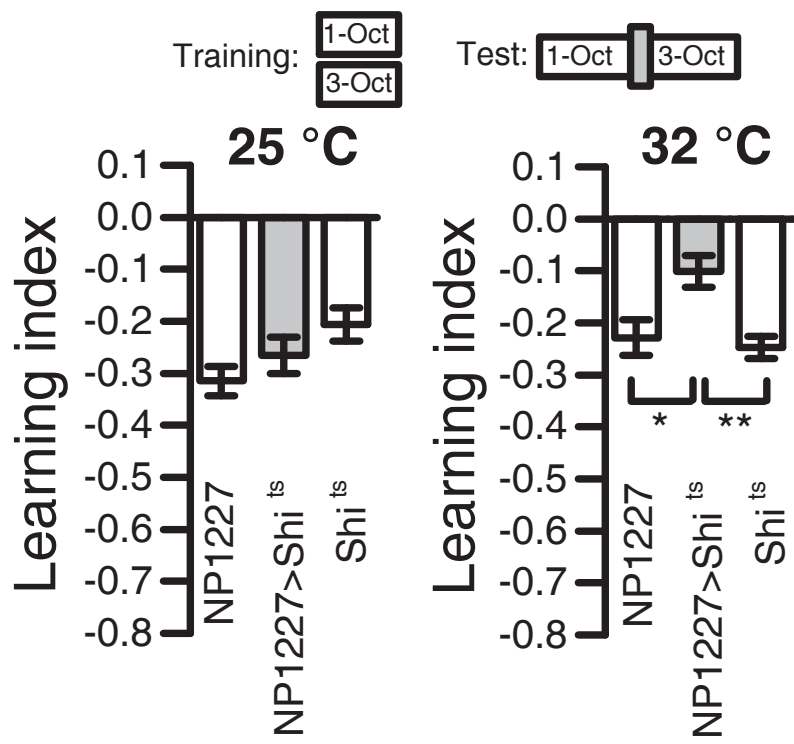

Figure 3.19. Differential training of similar odorants in NP1227>Shits flies. Approx. 100 flies with blocked synaptic transmission in LN1 neurons, and the genetic controls, were trained differentially with 1 -Oct and 3-Oct. One of the odorants served as CS+ whereas the other was presented as the CS-. At the permissive temperature of $25^{\circ} \mathrm{C}$, NP1227>Shits flies showed an avoidance of the punished odorant resulting in a learning index comparable to the genetic controls. However, at the restrictive temperature of $32{ }^{\circ} \mathrm{C}$, the avoidance of the negatively reinforced odorant was significantly reduced in NP1227>Shits flies compared to the genetic controls.

$\mathrm{n}=8-13$ each; mean \pm SEM

ANOVA with post hoc Bonferroni corrected t-test ${ }^{*}=p<0.05 ;{ }^{* *}=p<0.01$ similar odorants (3-Oct and 1-Oct) was paired with an electric shock $(\mathrm{CS}+)$ whereas the other was presented without reinforcement (CS-). In the subsequent test phase, the flies had to decide between the CS+ and the CS-.

Without the expression of shibire ${ }^{\text {ts }}$ at the permissive temperature, the flies of the test group (NP1227>Shits) avoided the negatively reinforced odorants (CS+) and decided for the arm of the T-maze where the CS- was presented. As depicted in figure 3.19, the resulting learning index was comparable with the genetic controls. At the permissive temperature, the control lines still showed a learned discrimination of the two similar odorants. However, NP1227>shits flies expressing the dominant mutant form of dynamin and thus with a blocked synaptic transmission in LN1 failed to discriminate between 1-Oct and 3-Oct during the test phase manifested by 


\section{Results}

a significantly lower learning index. This finding points towards a major function of type I inhibitory local interneurons in the discrimination process of similar odorants. Synaptic transmission of $L N 1$ is necessary to learn to discriminate 1-Oct and 3-Oct whereas it is not necessary to associate an electric shock with an odorant per se. Additionally, the discrimination of non-similar odorants is not affected by a block of synaptic transmission in $\mathrm{LN1}$ as shown in section 3.7.5.

Conclusively, the activity of type I inhibitory local interneurons in the antennal lobe is important for the discrimination of 1-Oct and 3-Oct. Without the release of GABA, the two similar odorants can not be distinguished any more and are not separated on a behavioral level after differential training.

\subsection{Different conditioning paradigms alter the olfactory preference}

Subsequently, it was of interest whether the training paradigm would influence the generalization of 1-Oct and 3-Oct in wild type flies. Differential training consists of the pairing of an odorant with an electric shock (CS+) and the presentation of another odorant (CS-). Absolute training, on the other hand, only consists of the presentation of the CS+ to the flies [Giurfa, 2004]. A neutral stimulus, i.e. mineral oil, is used as a CS-. A CS- is therefore not present in absolute training.

As visualized in Figure 3.20, differential training of 1-Oct and 3-Oct leads to an avoidance of the punished odorant as described before (gray bars, comparable to Figure 3.10. However, if the flies are not exposed to the CS- during the training and mineral oil is presented instead (absolute training, white bars), the avoidance of the punished odorant decreases significantly. The presentation of the CS- seems to be important for the flies to discriminate between 1-Oct and 3-Oct. After absolute training, the flies are discriminating between the two odorants but the ability to distinguish them is significantly reduced. It also has to be mentioned that pairing 1-Oct with the electric shock resulted in a better discrimination of the two odorants than pairing 3-Oct and the electric shock. 
Figure 3.20. Differential training increases odor discrimination. Groups of 100 flies were either conditioned with an absolute conditioning paradigm or a differential conditioning protocol. Differential training with one odorant serving as the CS+ (odor presentation paired with an electric shock) and the other as the CS- (no electric shock) leads to an avoidance of the punished odorant (gray bars) comparable to figure 3.10. If the presentation of the second odorant (CS-) is skipped and oil is presented instead as in the absolute training paradigm (white bars), the avoidance of the punished odorant decreases significantly.

$$
\begin{array}{r}
n=16 \text { each; mean } \pm \text { SEM } \\
\text { Two-sample t-test } \\
* *=p<0.01 ;{ }^{* * *}=p<0.001
\end{array}
$$

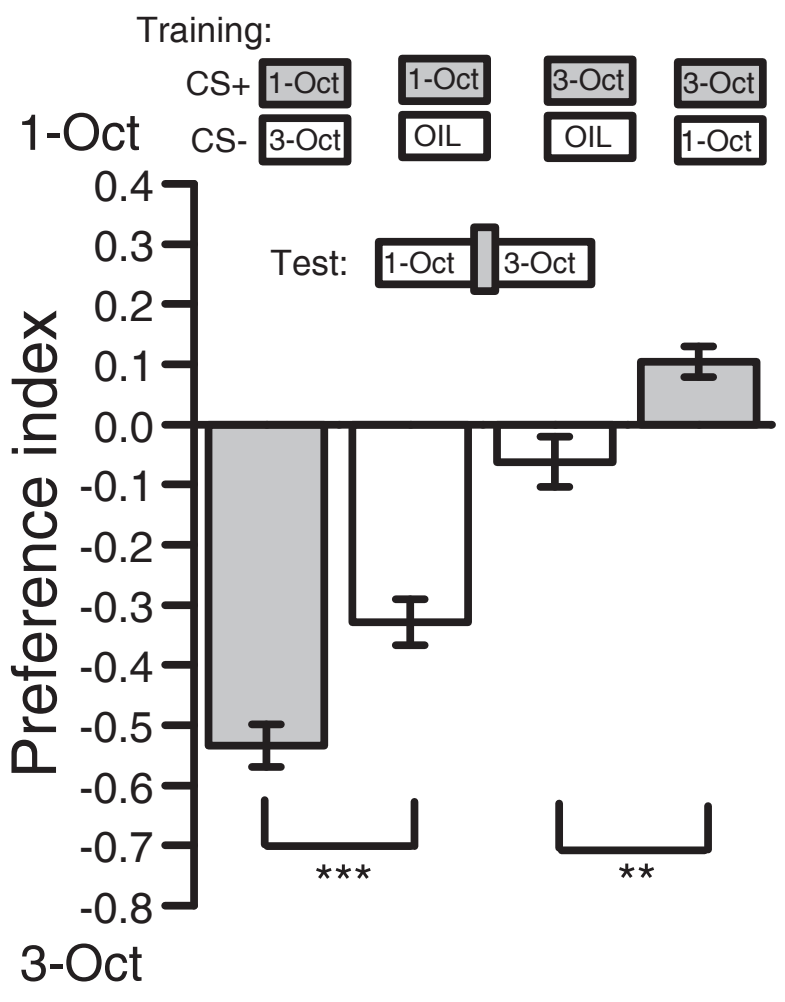

It can be concluded that the presentation of the CS- provides an important information during differential conditioning. Without the information that the CS- is explicitly not punished, a discrimination between similar odorants is not as pronounced. During absolute training, the $\mathrm{CS}_{+}$is associated with the punishment. This does not influence the generalization of the two odorants as they are still similar. The presentation of the CS- without the electric shocks provides the additional information that a difference between the odorants exists. Whereas one of them predicts a punishment, the other is predicting the absence of the punishment. This conclusion raises the question whether the timing of the CS- presentation with the training phase plays a role in the performance of the flies. 


\section{Results}

\section{Timing of CS- presentation has no obvious influence on learning}

During the 5 min training protocol for olfactory learning in the Tully-machine (see section 2.2.4.2), the electric shocks can be given at two time points. Either the first presentation of an odorant can be temporally paired with electric shocks and thereby become the CS+ or the second odorant can be associated with the punishment. Hence, the CS+ can be either presented before the CS- or afterwards. In order to investigate the effect of the timing of the CS- within the training protocol, the results of the experiments to figure out differences between absolute and differential training were subdivided into two groups. Each experimental group was tested equally often $(n=8)$ with a presentation of the CS- or CS+ as the first stimulus. A significant difference between the performances of flies receiving the $\mathrm{CS}+$ presentation prior to the CS- could not be found (Figure 3.21).

Hence, it seems not to be important if the presentation of the CS- is following or preceding the CS+. The information about the existence of a CS- must therefore be memorized during the training phase even if it is not known if a CS+ will follow.

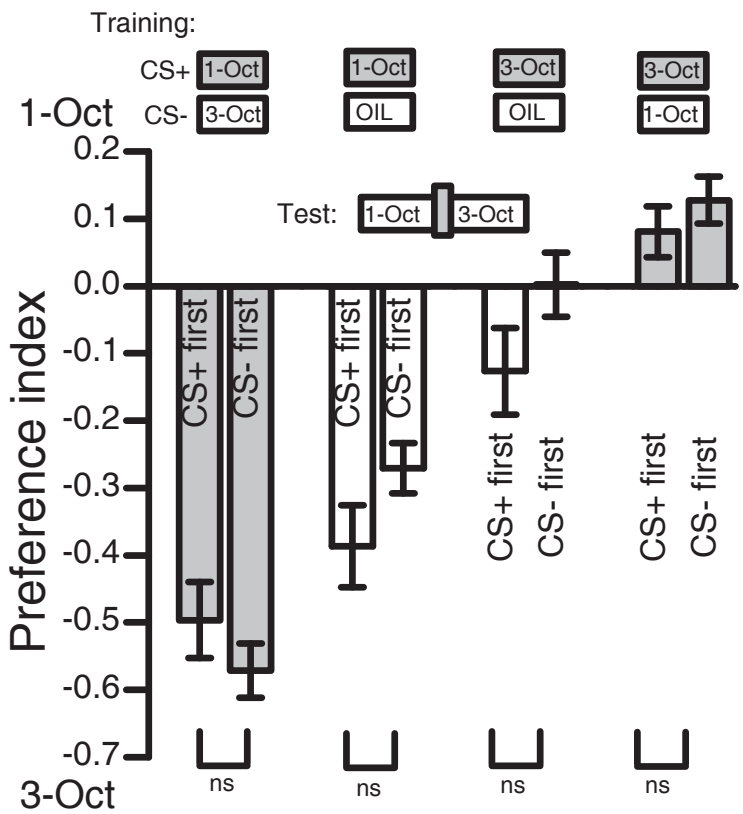

Figure 3.21. Timing of CS- presentation has no influence on the odorant preference after learning. During differential training, the electric shocks can be temporally paired with either the first or the second odorant presentation. Thereby, the CS+ can be either the first stimulus or the second. This timing of the CS+ (CS- respectively) did not have an influence on the performance of the flies. The preference indices of flies receiving the CS- before the CS+ are not significantly different from the indices of flies that received the $\mathrm{CS}+$ before the CS-. $\mathrm{n}=8$ each; mean \pm SEM Two-sample t-test 


\section{Results}

\subsection{Differential training reduces odor generalization}

During a next set of experiments, the influence of conditioned inhibition on discriminative training was investigated. Conditioned inhibition is one aspect of discriminative learning where the CS- is associated with the absence of a punishment and

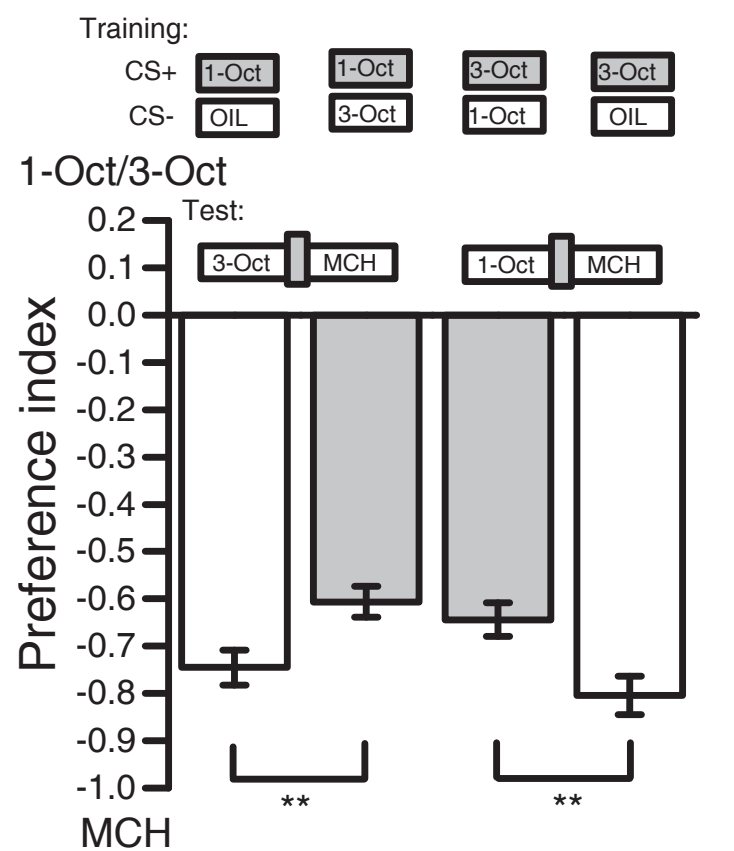

Figure 3.22. Differential training reduces odor generalization. The presentation of 1-Oct or 3-Oct, respectively, was paired with an electric shock (CS+) and trained against mineral oil as CS- (absolute training). During the subsequent T-maze test, the $\sim 100$ flies were exposed to $\mathrm{MCH}$ and the non-trained odorant (1-Oct in the case of pairing the electric shock $3-O c t$ and vice versa). As visualized in the white bars, the flies generalized between the odorants and avoided the similar odorant. When the odorants were trained differentially with one of the similar odorants serving asa a CS+ and the other as a CS- and the test comprised of the CS- and $\mathrm{MCH}$, the generalization was significantly reduced (gray bars).

$\mathrm{n}=16$ each; mean \pm SEM

Two-sample t-test; ${ }^{* *}=p<0.01$ opposite to the conditioned excitation where the $\mathrm{CS}_{+}$is associated with the punishment [Pavlov, 1927; Rescorla, 1969a b; Hearst \& Franklin, 1977; Savastano et al., 1999]. By training the flies absolutely with 3-Oct or 1-Oct against mineral oil and a subsequent test of two novel odorants $(\mathrm{MCH}$ and the untrained OCT), conditioned excitation is assessed as there can be no conditioned inhibition in the absence of a CS-. However, differential training of 1 -Oct versus 3 -Oct can result in conditioned excitation towards the CS+ and conditioned inhibition towards the CS-. As the subsequent test consisted of a novel odorant $\mathrm{MCH}$ and the CS-, the difference between absolute and differential training can only be explained by conditioned inhibition.

Indeed, after absolute training the flies avoided the untrained OCT in a choice against the novel odorant, $\mathrm{MCH}$ (Figure 3.22, white bars). This response can be explained by the strong generalization of 1-Oct and 3-Oct as described above (see Figure 3.7). However, differential training reduces the learning index and 


\section{Results}

thus the generalization. If 3-Oct is trained differentially against $1-O c t$ (3-Oct is CS+, 1-Oct is CS-) and subsequently 1-Oct is tested against $\mathrm{MCH}$, the generalization of the odorants is significantly reduced (and thus the discrimination increased). Similarly, the generalization is reduced upon differential training with 1-Oct as CS+, 3-Oct as CS- and a subsequent test of 3-Oct against $\mathrm{MCH}$. These results are depicted in the gray bars of Figure 3.22. During differential training, both conditioning traces (excitatory and inhibitory) are present.

It can be concluded, that the reduced generalization after differential training can be explained as an effect of conditioned inhibition. Therefore, the CS- plays a large role during differential conditioning and the acquired possibility to discriminate two similar odorants. Whereas absolute training only includes an association between a punishment and a sensory stimulus, differential training explicitly provides the information that two stimuli are different because one of them predicts a punishment whereas the other does not. It thereby emphasizes the importance to discriminate between the two similar stimuli even if they are very similar.

\subsection{Discrimination of similar odorants depends on the choice during the test}

The obtained results raised the question whether the presented odorants in the test situation are important for discrimination. In a subsequent set of experiments, 3-Oct and 1-Oct were trained differentially, but the test situation varied. When the CS+ was tested against mineral oil, the flies showed a strong avoidance of the odorant (Figure 3.23, striped graphs). However, during a test between mineral oil and the CS-, the avoidance of the odorant is decreased even though still prominent (Figure 3.23, solid graphs). This decrease was very prominent in the case of 1-Oct as CS+ and 3-Oct as CS-. This difference might be explained by the different receptors activated by the two odorants (see section 3.5). It is worth mentioning that the conditioned inhibition that associated the CS- with the absence of the shock was not strong enough to overcome the generalized conditioned excitation that associated the similar odorant with an electric shock. Therefore, mineral oil was preferred in the T-maze.

Additionally, the preference index of an odorant depended on its role during the train- 


\section{Results}

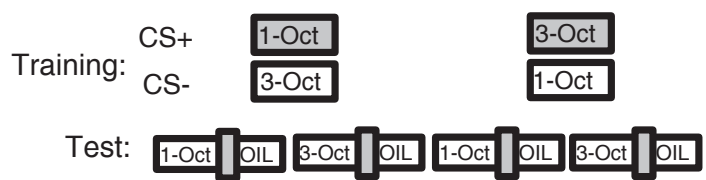

Odor

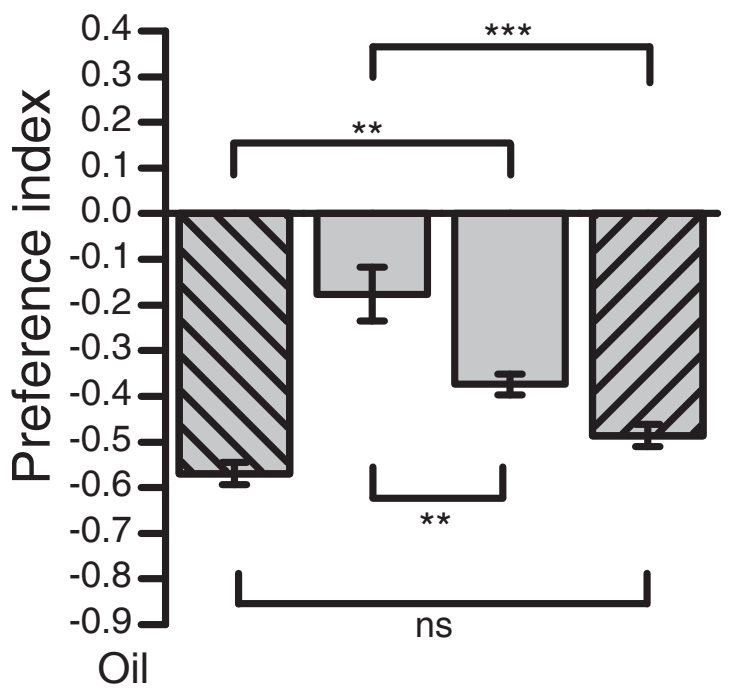

Figure 3.23. Discrimination of 3-Oct and 1-Oct depends on the presented odorants in the test situation. After differential training, the flies are avoiding the punished odorant (CS+) even if the CS- is not presented during the test (striped graphs). If the test situation consists of the CS- and mineral oil, the CSis still generalized and avoided even though considerably less (solid graphs). In addition, a significant difference can be observed between the responses towards the same odorant used as the CS- or CS+. Interestingly, the response to the CS- was also dependent on the odorant identity. 3-Oct as a CS- was significantly less avoided than 1-Oct as a CS- even though both odorants are avoided the same when serving as a CS+.

$$
\mathrm{n}=16 \text { each; mean } \pm \text { SEM }
$$

ANOVA with post hoc Bonferroni corrected t-test; ${ }^{* *}=\mathrm{p}<0.01 ;{ }^{* \star *}=\mathrm{p}<0.001$

ing. In line with previous experiments, the avoidance of an odorant that served as a $\mathrm{CS}+$ was significantly higher than the avoidance of the same odorant after training as CS-. Interestingly, the preference indices of the CS+S did not differ whereas the avoidance of the CS-s was significantly different. It can therefore be hypothesized that the conditioned inhibition of 3-Oct as a CS- with 1-Oct as a CS+ is stronger than the inhibition of 1-Oct as a CS- with 3-Oct as a CS+. Hence, the generalization after differential training is more reduced when 1-Oct acts as the CS+.

The test situation is an important factor during the discrimination of odorants. Even though differential training provides the information that the two similar odorants have to be discriminated, a discrimination can be difficult if only one of two similar stimuli is present at a time. Without the possibility to compare the two stimuli, a discrimination seems to be impeded.

Focusing on the learned discrimination of similar odorants, it was of particular interest which neuronal changes were underlying the behavioral results. It could be shown that type I inhibitory local interneurons are necessary for the discrimination 


\section{Results}

of two similar odorants (section 3.7). However, where the neuronal changes are located in the brain was not addressed. The mushroom body was shown to be the brain region responsible for the association of an odorant with a reinforcing stimulus by coincidence detection [Zars et al., 2000]. Hence, calcium imaging experiments were performed in the mushroom body in order to investigate possible changes in odorant evoked responses after learning. Therefore, calcium signals were measured before and after training of the fly under the microscope. Differential and absolute conditioning paradigms were applied in order to shed light on the differences between the two paradigms.

\subsection{Changes in neuronal activity in the mushroom body as a result to learning: first steps}

Neuronal activity in the mushroom body was assessed by measuring the changes in calcium concentrations in response to odorant presentation with GCaMP3.0 [Tian] et al., 2009]. As the $\beta$ ' and the $\gamma$-lobe were implicated in memory acquisition [Krashes et al., 2007; Qin et al., 2012] and dopaminergic neurons responsible for aversive conditioning innervate the $\gamma$-lobe [Aso et al., 2010; Qin et al., 2012; Waddell, 2013], a focal plane that includes the $\beta$ '- and the $\gamma$-lobe was selected. As an additional landmark, a protuberance in the $\gamma$-lobe could be identified (for a reconstruction of the mushroom body and the focal plane selected for calcium imaging, see figure 3.24 $A$ and $B$ ). An identification of the two lobes included in the experiment was possible due to a higher basal fluorescence observed in the $\gamma$-lobe. Changes in calcium concentrations as a correlate for neuronal activity was measured in response to three pseudo-randomized presentations of the two similar odorants 1-Oct and 3-Oct and the control odorant $\mathrm{MCH}$ (for a scheme of the whole imaging procedure, see 2.4.) Signals could be observed for all three odorants in the $\beta$ '-lobe and in the medial regions of the $\gamma$-lobes, referred to by Tanaka et al. [2008] as $\gamma 1, \gamma 2$ and $\gamma 3$ (falsecolor coded images of an example fly are depicted in figure $3.24 \mathrm{C}$ ). The average odorant responses of the whole mushroom body for the example fly during odorant presentation are depicted in figure 3.24 D. The elicited responses were consistent for each of the three odorant presentations. In order to compare the spatial activ- 


\section{Results}
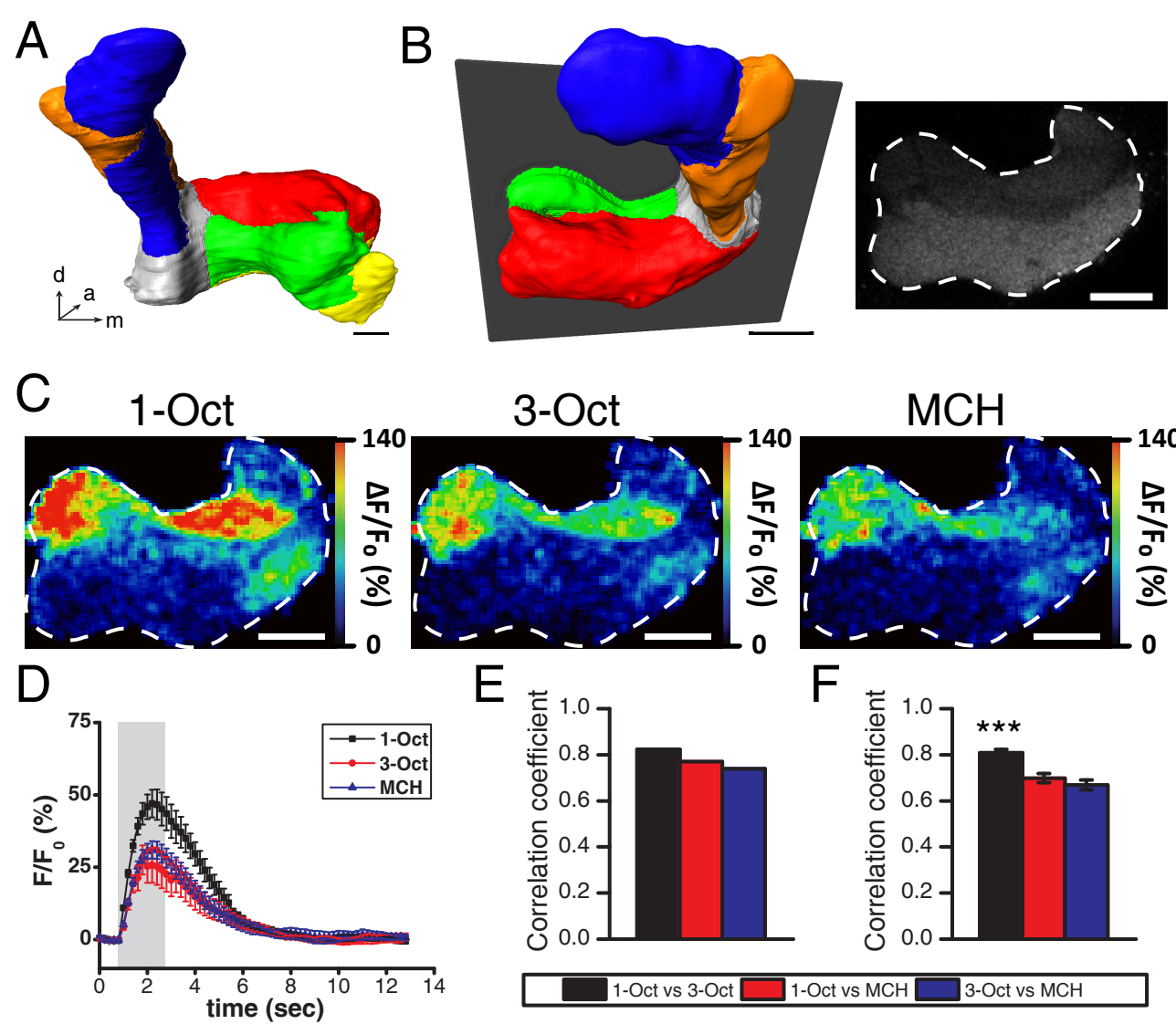

E

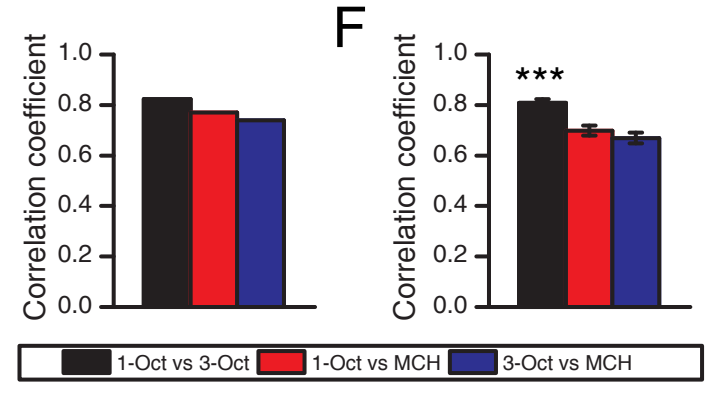

Figure 3.24. Neuronal activity pattern in the mushroom body.

(A) A 3D-reconstruction of the mushroom body shows the different lobes (see section 1.4.3 and figure 1.5. $\mathrm{d}=$ dorsal; $\mathrm{a}=$ anterior; $\mathrm{m}=$ medial $(\mathrm{B})$ The focal plane for the imaging experiments is depicted in gray. Calcium activity could therefore be measured from the $\gamma$ and the $\beta$ '-lobes. The two lobe regions were differentiated by the difference in the basal fluorescence as shown on the right side (the $\gamma$-lobe consistently showed a higher basal fluorescence than $\beta^{\prime}$ ). (C) False-color coded images of the evoked calcium responses during odorant presentation. (D) Average responses from three calcium measurements with the same odorants in one fly in the whole mushroom body. (E) Pixel based correlation coefficient calculated from the average odorant responses for the three possible combinations in one fly. $(\mathbf{F})$ The correlation coefficient between the two similar odorants 1-Oct and 3-Oct from 20 measured flies showed a significantly higher correlation compared to the correlation between 1-Oct and $\mathrm{MCH}$ (and 3-Oct and $\mathrm{MCH}$, respectively).

Scale bar $=20 \mu \mathrm{m}$

Friedman ANOVA with post hoc Bonferroni corrected paired sample Wilcoxon signed rank test; ${ }^{* * *}=p<0.001$ 


\section{Results}

ity patterns evoked by the odorants, the correlation between the patterns for each possible odorant pair was calculated from the average responses for each fly (the correlation coefficients of the example fly are shown in figure $3.24 \mathrm{E}$ ). Comparable with the responses evoked in the antennal lobe (see section 3.5), a correlation between 1-Oct and 3-Oct could also be observed in the mushroom body. The neuronal activity patterns elicited by 1-Oct and 3-Oct from 20 measured flies before any conditioning showed a stronger correlation than the pattern evoked by 1-Oct and $\mathrm{MCH}$ and 3-Oct and $\mathrm{MCH}$, respectively (Figure 3.24 F). It can therefore be stated that the similarity of the two odorants that was observed on the behavioral level and on the physiological level in the antennal lobe is conveyed further to the mushroom body. For the subsequent analysis of possible conditioning dependent changes, the mushroom body was subdivided in two regions. As described above, odorant evoked signal could be observed in the $\beta$ '-lobe and in the $\gamma 1, \gamma 2$ and $\gamma 3$-regions of the $\gamma$-lobe which will be referred to as the $\gamma$-shaft from now on (Figure $3.25 \mathrm{~A}$ ). Both regions elicited a signal during odorant presentation in all measured flies $(n=20)$ as depicted in figure $3.25 \mathrm{~B}$ and $\mathrm{C}$. It has to be mentioned that the amplitude of the signal varied between flies. Especially the activity in the $\gamma$-lobe was very variable. The 20 flies that were imaged during this experiment were further subdivided into two groups. One group received an absolute training with either 1-Oct or 3-Oct (5 flies each) as a CS+ and mineral oil was presented instead of a CS-. The second group was trained differentially either with 1 -Oct as the CS+ and 3-Oct as the CS- (5 flies) or the other way around (5 flies). The training procedure was the same as during the behavioral experiments with a temporal pairing of the CS+ with 12 electric shocks within one minute and the CS- presented for one minute without reinforcement (see schemes in figure $3.25 \mathrm{D}$ and $\mathrm{E}$ ). Different effects on the correlation coefficients (as measure for odorant similarity [Svedlow et al., 1976]) could be observed depending on the training paradigm applied. Absolute training on the one hand resulted in a significantly higher correlation of 1 -Oct and 3-Oct in the $\beta^{\prime}$-lobe whereas no effect could be observed in the $\gamma$-shaft region (Figure $3.25 \mathrm{D}$ ). In contrast, differential training did not change the correlation of the two similar odorants in the $\beta$ '-lobe but a significant decrease in correlation in the $\gamma$-shaft region (Figure $3.25 \mathrm{E}$ ). Note that the variation of the correlations calculated for the distinct lobes was different. The variability of the correlations in the $\beta^{\prime}$-lobe was rather low but for the $\gamma$-shaft they were 

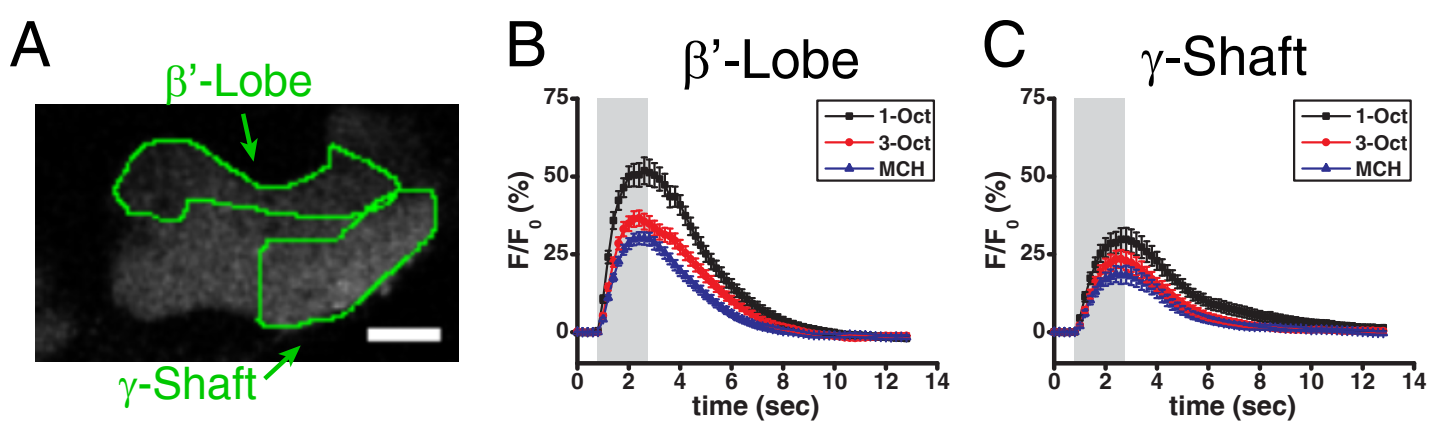

D
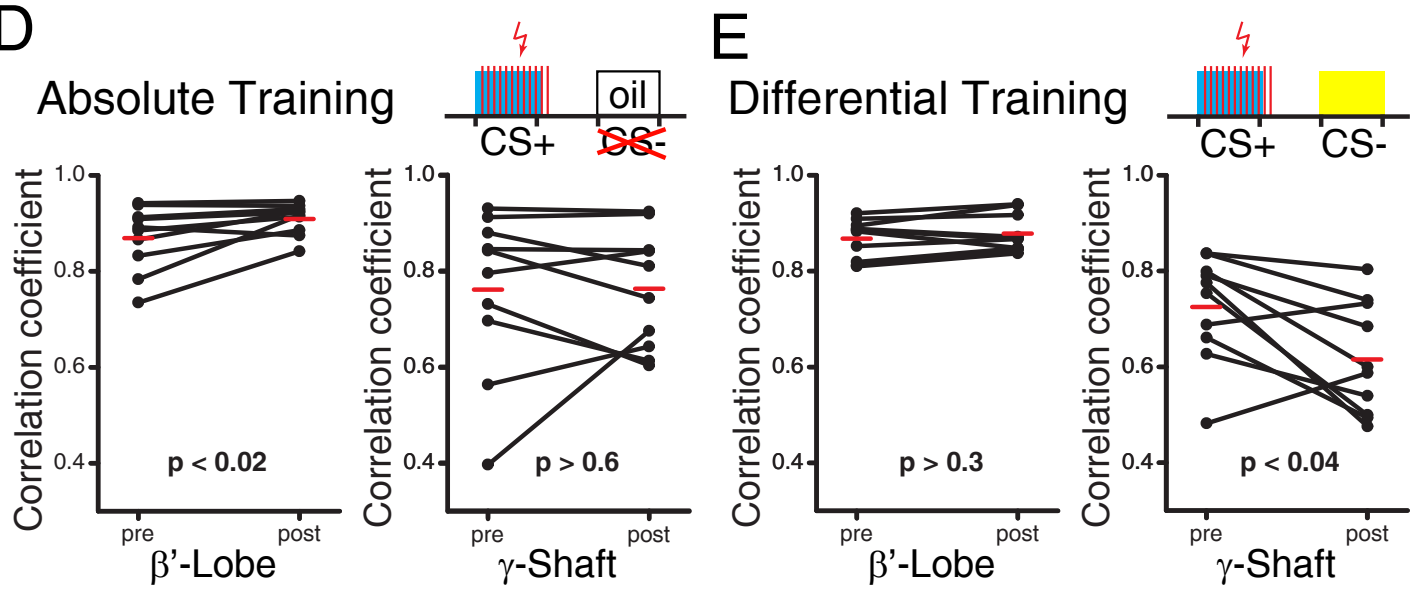

Figure 3.25. Learning evoked changes in odorant representation in the mushroom body.

The mushroom body was subdivided into two regions for the analysis. (A) One region consisted of the $\beta$ '-lobe and the other the shaft of the $\gamma$-lobe. These two regions were chosen by the odorant evoked calcium signals observed during stimulation depicted in figure 3.24 C. (B,C) Averaged time courses of evoked calcium signals before conditioning from 20 flies. (D) Comparison of maximal pixel based correlation coefficients of activation patterns evoked by 1-Oct and 3-Oct in the two regions in 10 flies after absolute training. A significant increase of the correlation between the two similar odorants can be observed in the $\beta$ '-lobe whereas the $\gamma$-shaft region did not show a significant effect of absolute training. (E) Comparison of maximal pixel based correlation coefficients of activation patterns evoked by 1-Oct and 3-Oct in the two regions in 10 flies after differential training. No change in the $\beta$ '-lobe could be detected. In the $\gamma$-shaft, a significant decrease of correlation reflecting a stronger dissimilarity after the training procedure is prominent. Red bars in (D) and (E) represent the average correlation coefficient. $\mathrm{n}=10$; paired sample Wilcoxon signed rank test 


\section{Results}

varying stronger. An explanation for this difference can be attributed to the difference in amplitude of the elicited signals. Indeed, the signals obtained in the $\beta^{\prime}$-lobe were consistently higher than in the $\gamma$-shaft region. A further analysis of the outliers observed in figures $3.25 \mathrm{D}$ and $\mathrm{E}$ revealed that the outliers are produced by flies with very low signals in the respective regions. An exclusion of the outliers from the statistical analysis did not change the overall outcome of the analysis.

In conclusion, the physiological similarity of 1-Oct and 3-Oct that could be observed with $\mathrm{Ca}^{2+}$-imaging in the antennal lobe is conveyed to the mushroom room body lobes and results in a higher correlation of the elicited neuronal responses. This correlation between the activation pattern elicited by 1 -Oct and 3 -Oct could be further increased in the $\beta^{\prime}$-lobe after absolute training. Conversely, differential conditioning resulted in a decreased correlation of the evoked pattern in the $\gamma$-shaft region of the mushroom body.

\subsection{New tools for future studies: generation of new fly strains for optical imaging}

In addition to the above mentioned experiments on perceptual and physiological odorant similarity, three new fly strains were generated during the progress of this thesis. All of them are expressing calcium sensors under the control of different promotors. A red fluorescent calcium sensor can be used in combination with green fluorescing proteins and is therefore valuable for future studies. The currently developed red calcium sensor R-GECO1.0 [Zhao et al., 2011] was cloned in two different fly injection vectors: pCaSpeR:mb247 [Riemensperger et al., 2005] and pUAST [Brand \& Perrimon, 1993]. Additionally, a fly strain with expression of the green fluorescing calcium sensor GCaMP3.0 under the control of the lexOp promotor was generated. With the LexA/LexAop system [Szüts \& Bienz, 2000; Lai \& Lee, 2006], these flies can be used to drive GCaMP3.0 expression in specific cell groups without using the more common binary GAL4/UAS system. Hence, the GAL4/UAS system can be used independently to manipulate neurons while the expression of GCaMP3.0 to monitor neuronal activity is regulated with the LexA/LexOp-system. 


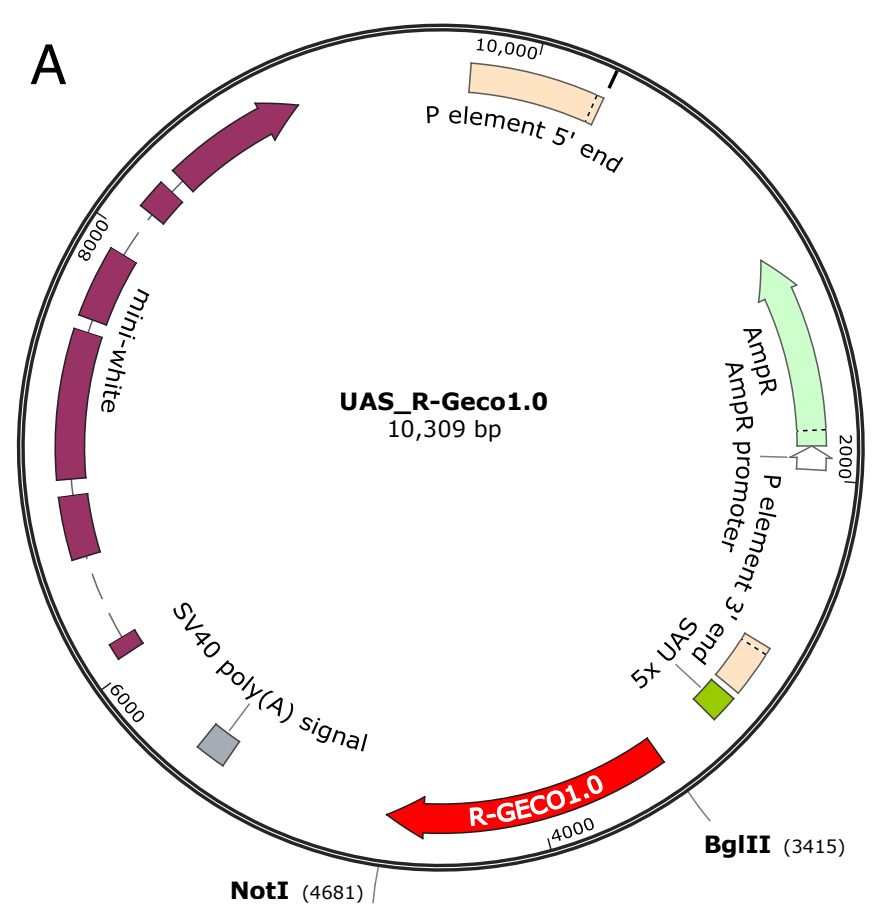

Figure 3.26. Vectormaps of UAS:R-GECO1.0 and mb247:R-GEC01.0.

Both vectors with R-GECO1.0 insertion include the two necessary p-elements to inserted the flanked DNA into the Drosophila genome (creme color). In light green, the ampicillin resistance for vector amplification in $E$. coli is depicted in both vectors. Additionally, the mini-white gene (in UAS:R-GECO1.0) or the white locus (in mb247:R-GECO1.0) colored in purple was present to identify flies with a positive germline transformation by their eye color. (A) The UAS:R-GECO1.0 vector contains five times the UAS region (dark green) as GAL4 binding sites. Five copies increase the binding probability of GAL4 and thereby the expression of the transgene. As the gene for R-GECO1.0 (red) is located behind the UAS regions, the expression of the protein is limited to cells with GAL4 expression. (B) The mb247:RGECO1.0 vector contains the mb247-promotor (dark green) upstream of R-GECO1.0 (red) to drive expression specifically in kenyon cells in the mushroom body Riemensperger et al., 2005. 


\section{Results}

\subsubsection{Expression of red fluorescing calcium indicators}

The pUAST backbone vector includes five upstream activator sequence (UAS) regions as binding partners for GAL4 induced expression [Brand \& Perrimon, 1993]. Downstream to the UAS binding site, the R-GECO1.0 DNA was cloned between the Notl and Bglll restriction sites. In addition, the mini-white gene was present in the p-element flanked region (Figure $3.26 \mathrm{~A}$ ).

The p-elements are necessary for the insertion of the enclosed DNA into the Drosophila genome after vector injection in the germline cells [Rubin \& Spradling, 1982]. Mini-white is used as a marker for positive germline transformation. The plasmids are injected into flies with a defective white gene and therefore a white eye color. Only flies with an insertion of the transgenic DNA express the mini-white gene and have eyes with a red color [Hazelrigg et al., 1984].

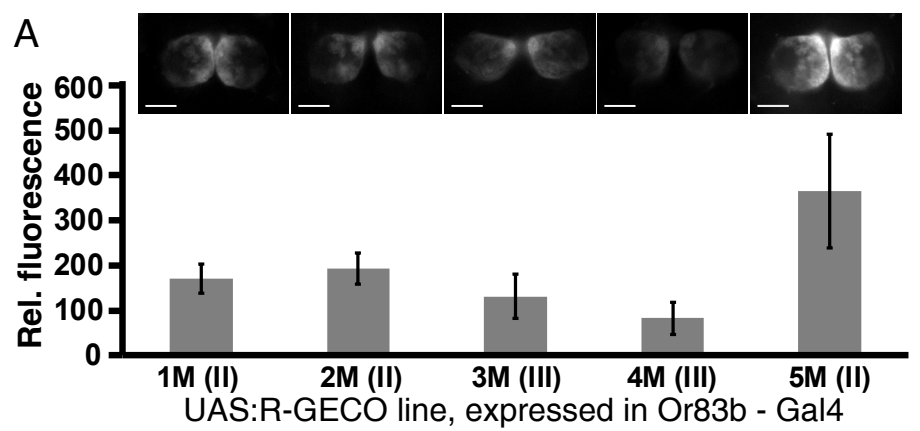

B

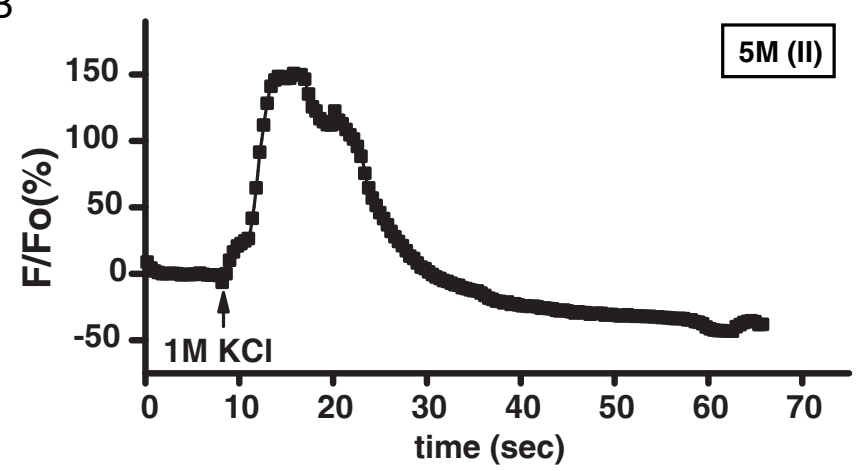

Figure 3.27. Evaluation of the expression levels of the UAS:R-GEC01.0 fly lines.

(A) The UAS:R-GEC01.0 flies were crossed with Or83bGAL4 in order to investigate the expression levels of the different lines. After the dissection of the brain, the expression levels of the transgene were tested by measuring the basal fluorescence. The average measured fluorescence from five flies per line differed in the five versions of UAS:RGECO1.0. The line 5M located on the second chromosome showed the highest basal expression. (B) The functionality of the sensor was confirmed in an imaging experiment in a living fly of line $5 \mathrm{M}$. Pipetting $\mathrm{KCl}$ on the brain resulted in a depolarization of the neurons and thereby an increased calcium concentration in the cell which could be detected by the sensor.
Scale Bar: $50 \mu \mathrm{m}$ 


\section{Results}

The pCaSpeR:mb247 backbone vector did not contain the mini-white gene but the white-locus to express the white gene for red eye-color in positively transformed flies. Instead of the five UAS regions, the vector included the mb247promotor for specific expression of the transgene in Kenyon cells of the mushroom body [Riemensperger et al., 2005] (Figure 3.26 B).

During the amplification of the vectors in $E$. coli, the ampicillin resistance was necessary for the selective amplification of transfected clones. The resistance casette is located outside of the p-elements and therefore not incorporated in the Drosophila genome after injection. The vectors were injected into the germline cells by the company BestGene Inc. (Chino Hills, CA, USA) which also determined the chromosome on which the transgene was incorporated into the genome. After shipment of the flies to Germany, the expression of the transgenes was tested by Ulrike Pech in the laboratory.

The UAS:R-GECO1.0 line was combined with a driver line targeting olfactory sensory neurons, Or83b-GAL4, in order to express the calcium sensor. First, the base line fluorescence without neuronal activation was measured in five different fly strains with UAS:R-GECO insertions. Therefore, five brains per strain were dissected and the basal fluorescence averaged (3.27 A). All five lines expressed R-GECO1.0, but the line $5 \mathrm{M}$ with the insertion of the transgene on the second chromosome showed the highest basal fluorescence. This line was used to examine the functionality of the calcium sensor. Therefore, $\mathrm{KCl}$ was pipetted on the brain which leads to a depolarization of the neurons. The increase of the fluorescence shown in figure 3.27 B confirmed the functionality of the sensor. The measured signal $\left(\frac{\Delta F}{F_{0}}\right)$ increased by $150 \%$ of the base line fluorescence $\left(F_{0}\right)$. Hence, the functionality of the new UAS:R-GECO1.0 fly line could be confirmed.

Similarly, the functionality of mb247:R-GECO1.0 was examined. After determining the line with the highest basal fluorescence, the response to $\mathrm{KCl}$ stimulation was observed. As shown in figure 3.28, the signal in the mushroom evoked by $\mathrm{KCl}$ application was even stronger than in the antennal lobe. Whereas the UAS line expressed in the olfactory sensory neurons showed an increase in fluorescence of $150 \%$ compared to the base line $\left(\frac{\Delta F}{F_{0}}\right)$, the fluorescent increase in the mb247 line was $350 \%$. 


\section{Results}

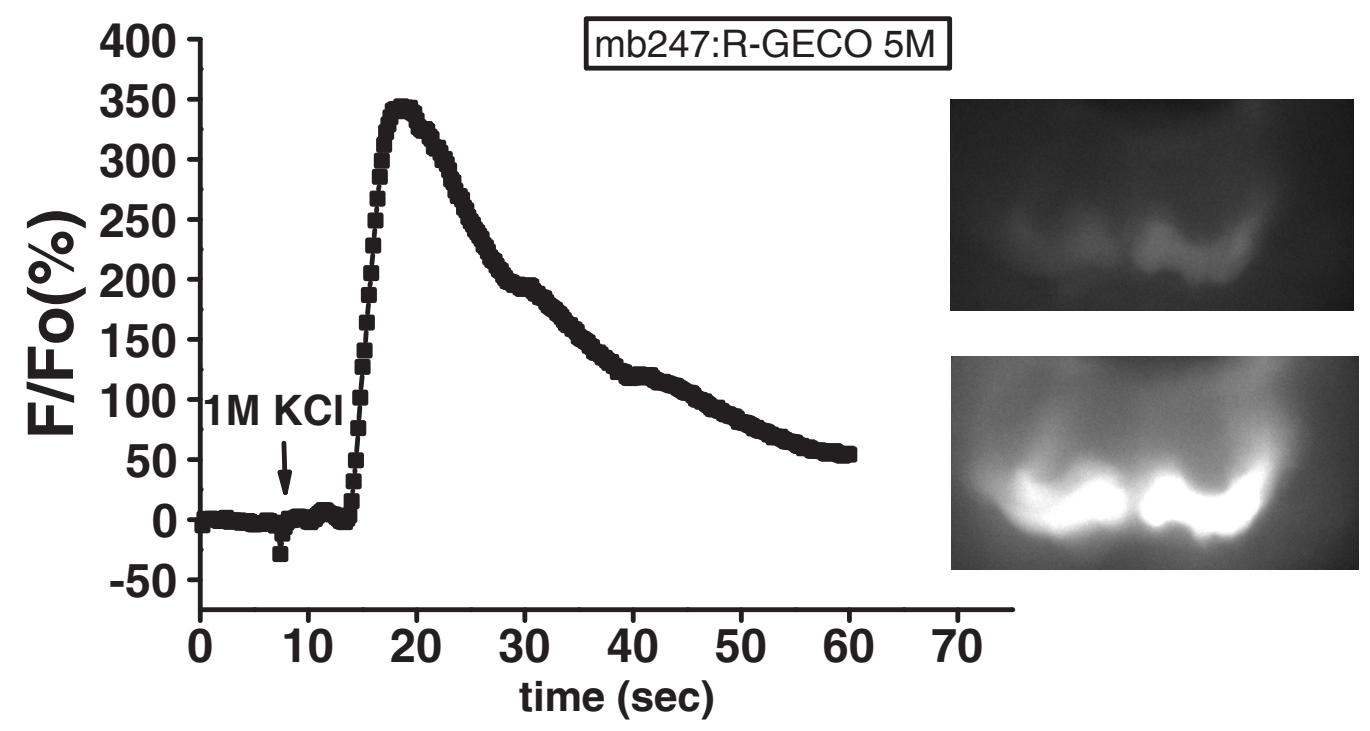

Figure 3.28. Evaluation of mb247:R-GECO1.0.

Flies expressing the red calcium sensor R-GECO1.0 under the control of the mb247 promotor express the sensor in the mushroom body. Upon stimulation with $\mathrm{KCl}$, the fluorescence of the sensor increases by $350 \%$. The two pictures on the right show the fluorescence of the mushroom body before and during the stimulation with $\mathrm{KCl}$.

Conclusively, it could be shown that the new red fluorescent calcium sensor RGEC01.0, developed by Zhao et al. [2011] is functional in the fruit fly. The generated fly lines expressing R-GECO1.0 under the control of UAS therefore provide a new tool to investigate neuronal function. With the help of various GAL4-driver lines, the sensor can be expressed in subsets of neurons and used in combination with commonly used green fluorescent proteins to label other structures of interest. It will even be possible to monitor the neuronal activity in two distinct subsets of neurons when the red sensor is used in combination with a green sensor. For example, the other newly generated line expressing R-GECO1.0 under the control of the mb247 promotor can be used to image neuronal activity in the mushroom body and any subset of neurons targeted by the GAL4 line of choice. Additionally, it will be possible to use UAS:R-GECO1.0 in combination with GCaMP3.0 under the control of another binary expression system, e.g. the LexA/LexOp system [Szüts \& Bienz, 2000; Lai \& Lee, 2006. Therefore, the third newly generated fly line, LexOp:GCaMP3.0, will be of use. 


\section{Results}

\subsubsection{A more versatile application of the GCaMP3.0 sensor}

The generation of a fly line expressing the calcium sensor GCaMP3.0 under the control of the LexOp promotor provides an imaging tool independent of the commonly used GAL4/UAS system. However, the expression of the calcium sensor is still not restricted to a single subset of neurons. The independent binary system LexA/LexOp can target the expression to a distinct subset of cells. A cell specific promotor drives the expression of LexA which in turn binds to the LexOp operator and enables the expression of the transgene (i.e. GCaMP3.0)[Szüts \& Bienz, 2000]. A detailed description of the Lex$\mathrm{A} /$ LexOp system can be found in section 1.3.1.

The vector pLOT W_GCaMP3.0 contains the two $p$-elements necessary for incorporation of the transgene into the Drosophila

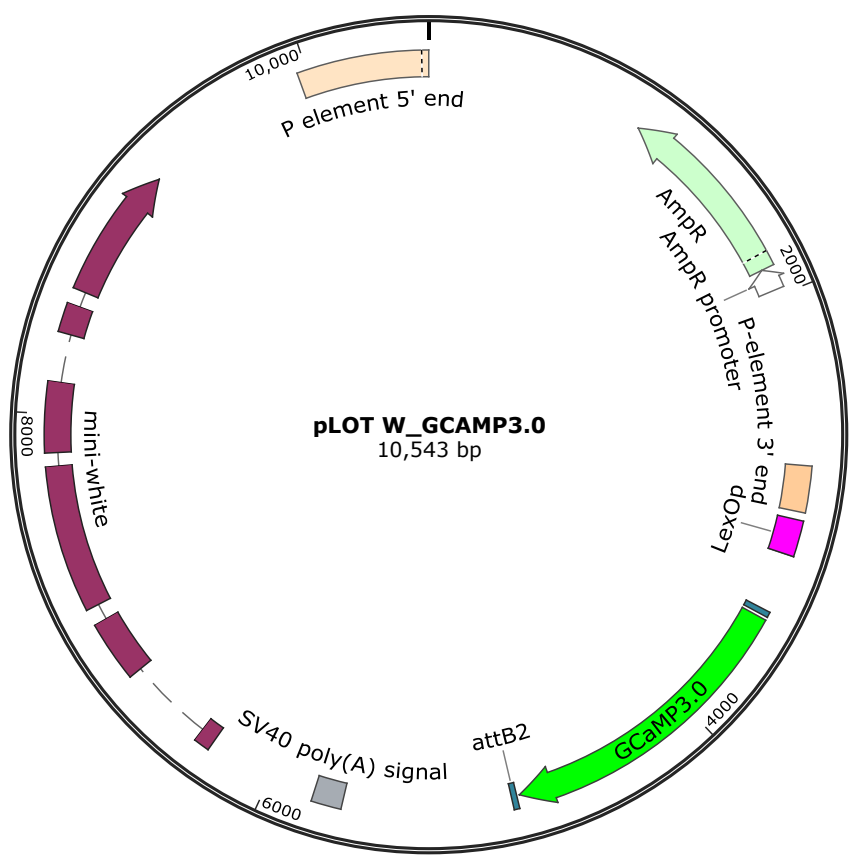

Figure 3.29. Vectormap of LexOp:GCaMP3.0.

The LexOp operator (magenta) is located before the GCaMP3.0 gene (green) to ensure an expression of the transgene specifically in cells with an expression of LexA. Additionally, the pLOT W_GCaMP3.0 vector contains the mini-white gene (purple) to identify flies with a positive germline transformation by their eye color. These loci (LexOp, GCaMP3.0 and miniwhite) are flanked by the p-elements (creme) to be inserted in germline cells. The vector additionally included an ampicillin resistance casette (light green) for selection of transfected cells during the cloning process.

genome after germline injection [Rubin \& Spradling, 1982]. In between, the LexOp operator upstream of the GCaMP3.0 sequence and the mini-white gene are located. Additionally, the vector contains an ampicillin resistance in order to select only transfected $E$. Coli during amplification. 


\section{Results}

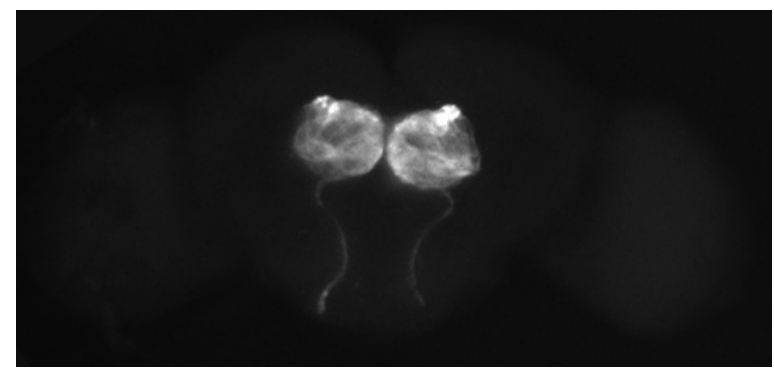

Figure 3.30. Expression of LexOp:GCaMP3.0 in Or83blexA:VP16 flies.

The generated LexOp:GCaMP3.0 flies were combined with the Or83b-lexA:VP16 driver line in order to test for an expression of the transgene. After dissection of the brain, the fluorescence was measured under the miscroscope. A strong signal could be observed in the antennal lobes which confirmed the expression of a functional GCaMP3.0 protein in the olfactory sensory neurons targeted by the Or83b-lexA:VP16 line.

The different versions of the new fly strain were characterized together with Master student Angelika Schulz. The LexOp reporter lines were combined with a LexAdriver line targeting the olfactory sensory neurons, Or83b-LexA:VP16 [Lai \& Lee, 2006]. Expression of the sensor was observed after brain dissection (Figure 3.30) and the lines with the highest expression were determined.

Unfortunately, the generation and characterization of the new fly strains was time consuming and was finished only after the imaging experiments for this Thesis had to be started. Additionally, the experimental design did not require a red calcium sensor and was independent of a binary expression system. A fly line with a stable, homozygous expression of a calcium sensor in the mushroom body without further combination steps was needed. Therefore, the investigation of neuronal activity in the mushroom body was performed with the mb247-GCaMP3.0 line already established in the laboratory. 


\section{Discussion}

The adaptation of behavioral responses is an important ability of animals as a response to changing environmental stimuli. During its life, an animal is exposed to a variety of external stimuli and experiences the consequences following these stimuli. It thereby forms associations between the stimulations and a beneficial or harmful situation that is temporally paired. It learns to alter its responses depending on these experiences. Additionally, a variation of the perception of the sensory input might be of advantage if the prediction of a reward or a punishment is dependent on very distinct stimuli or several stimuli predict the same consequence. An adaptation of the olfactory acuity would represent such a variation of the perception. In the present study, the ability of the fruit fly Drosphila melanogaster to perform such tasks was investigated. In a first approach, sensory preconditioning was used to decrease the perceived difference between two dissimilar odorants by forming an association between them.

\subsection{Sensory preconditioning - an attempt to investigate changes in olfactory acuity}

The sensory preconditioning paradigm [Brogden, 1939; Kimmel, 1977] was used to investigate a possible change in behavioral olfactory acuity in fruit flies. The question whether it is possible to decrease the olfactory acuity by a temporal pairing of two dissimilar odorants $A$ and $B$ in a mixture was addressed. Does this temporal pairing result in the formation of an association between the odorants? In order to test this hypothesis, the flies were subjected five times with the odorant mixture before odorant $\mathrm{A}$ was negatively reinforced with electric shocks and a novel odorant $\mathrm{C}$ presented without any reinforcement. In the subsequent test for learning, odorant $C$ 


\section{Discussion}

was tested against odorant $B$ (see figure $3.3 \mathrm{~A}$ ). Even though sensory preconditioning could be shown in flies before [Brembs \& Heisenberg, 2001], the present study could not find any preconditioning effect. In the study from Brembs \& Heisenberg [2001], visual stimuli were used and a pattern was associated with a color. Even though both stimuli are visual, the underlying circuits during visual processing involve different photoreceptors and pathways [Kandel et al., 2000]. Therefore, the association of a pattern with a color is a much easier task than the association of two odorants. Additionally, one might assume that an association between a color and a pattern is a more relevant information for a fruit fly than the association of two unnatural monomolecular odorants.

However, previous experiments in honey bees could show sensory preconditioning of olfactory monomolecular stimuli [Müller et al., 2000]. Besides the fact that bees have a more complex nervous system than flies, two major differences between this study and the present study can be found: whereas Müller et al. [2000] used pure odorants and an appetitive and absolute conditioning paradigm, the present study used diluted odorants (1:5, 1:20 and 1:33 respectively) and an aversive and differential conditioning paradigm. It might therefore be possible that an association between the odorants presented during the preconditioning phase to a configural unit as described by Müller et al. [2000] in reference to Pearce [1994] can only be achieved with higher odorant concentrations and an appetitive conditioning paradigm. Additionally, the differential conditioning used in the present study explicitly trains the flies to discriminate between two odorants (see section 3.9). A prior association between two very dissimilar odorants (see [Niewalda et al., 2011]) might not be strong enough to overcome the forced discrimination. Another possible explanation is of course, that the fly is not capable of olfactory sensory preconditioning per se.

As it was not possible to decrease the olfactory acuity of Drosophila and thereby make two dissimilar odorants more similar, the opposite approach was used during further experiments. The similarity of two structurally and chemically similar odorants was decreased and thereby the olfactory acuity increased. 


\subsection{The chemical similarity of $1-$ Oct and $3-$ Oct results in a behaviorally and physiologically determined similarity}

It could be shown that the structurally similar monomolecular odorants 1-Oct and 3-Oct are also perceived as similar by the flies. Even though one might argue that this finding is intuitive, the molecular similarity of an odor does not necessarily imply a perceptual similarity [Haddad et al., 2008; Schmuker \& Schneider, 2007]. However, on a behavioral level, an absolute training of one of the odorants resulted in an avoidance of not only the trained odorant but also the similar odorant. This strong generalization could not be observed for the dissimilar control odorant $\mathrm{MCH}$ (see Figure 3.7).

To test the similarity of the two odorants on a physiological level, calcium imaging experiments were performed in the antennal lobe and in the mushroom body horizontal lobes. The increase of the fluorescence of the genetically encoded calcium sensor GCaMP3.0 [Tian et al., 2009], and thereby an increase in the intracellular $\mathrm{Ca}^{2+}$ concentration, was used as a measure of neuronal activity.

In the antennal lobe, the activity of olfactory sensory neurons in response to odorant presentation was observed. The activity patterns evoked by 1-Oct and 3-Oct showed a strong similarity as almost the same glomeruli responded with an increase of fluorescence in the two focal planes monitored. The detected difference between 1-Oct and 3-Oct was primarily found in an additional glomerulus (VL2) activated by 1-Oct. Additionally, the shapes of the time courses of activation differed minimally between 1-Oct and 3-Oct in glomeruli that were activated by both odorants (see figures 3.8 and 3.9. However, these differences in the response curves were not investigated further. As the analysis was only restricted to two focal planes, additional glomeruli might be activated by the odorants that were not addressed during this study.

In the light of the inability of flies to discriminate similar odorants without a functional synaptic transmission of type I inhibitory local interneurons (LN1) as described in section 3.7, it might be interesting to investigate the glomerular activation patterns evoked by 1 -Oct and 3-Oct without functional LN1 neurons. It would be interesting 


\section{Discussion}

to see if the activation patterns become even more more similar. This investigation could be further extended by including a measurement of the second order neurons in the antennal lobe, the projection neurons. Additionally, a possible change of activation in response to different conditioning paradigms with and without functional output from inhibitory local interneurons could be investigated. However, as mentioned above, the problem of different focal planes and the question where to look for changes resides. It is not elucidated if a block of synaptic transmission or learning induced changes would alter the response of a single glomerulus or have an influence on the recruitment or silencing of additional glomeruli.

In the mushroom body, odorant evoked responses were measured in the intrinsic neurons, the Kenyon cells. The acquisition of imaging data was restricted to the horizontal lobes. As the odor specific spatial map of the antennal lobe was shown to be relayed to the mushroom body calyx [Lin et al., 2007], it can be assumed that a spatial similarity of the patterns evoked by 1-Oct and 3-Oct at the level of the calyx would be maintained. However, it was not clear if the similarity is further encoded in the sparse activation of Kenyon cells. As the horizontal lobes have been shown to be involved in early memory acquisition and consolidation [Waddell, 2013], it was of particular interest to focus the imaging studies on this region.

Even though the analysis of the odorant evoked pattern in the mushroom body has proven to be not as straight forward as in the antennal lobe, a similarity of the two odorants 1-Oct and 3-Oct could be shown. The similarity was measured by calculating the correlation between the odorant evoked activity patterns. To be able to do so, it was crucial not to lose spatial information during image processing. Therefore, it was not possible to use any spatial filter to reduce noise as during the standard procedure. Additionally, a correction for movement of the fly brain in the $x$ - and $y$-direction is crucial after imaging experiments. The alignment used for the antennal lobe based on the TurboReg plugin for ImageJ [Thévenaz et al., 1998]. However, this plugin already uses a spatial filter during the alignment. Therefore, a new alignment tool had to be applied that was found in the literature [Guizar-Sicairos et al., 2008] and could be downloaded as a routine for MatLab. A subsequent reduction of noise was done with a non-spatial Kalman-Filter Kalman, 1960]. It is possible that temporal information might be affected by the use of this filter, but the spatial information of the images stayed intact. 


\section{Discussion}

The aligned and noise-reduced time series of calcium activity in the horizontal lobes were subjected to a pixel-based analysis during which the change of the fluorescence intensity at each time point was calculated for each pixel $\left(\frac{\Delta F}{F_{0}}\right)$. This preprocessing of the data made it possible to compare the activation pattern of three different odorants (1-Oct, 3-Oct and $\mathrm{MCH}$ ) in one fly. The correlation coefficient between the images at any given time point is an indicator for the similarity of the pattern at that time point [Svedlow et al., 1976]. The maximal correlation coefficient between two odorants within the whole time series of images was taken to define the similarity of this specific odorant pair. As the correlation coefficient is a relative measure, a comparison between flies could be made even though individual flies showed distinct activation patterns depending on the exact focal plane and the level of expression of the calcium indicator. A statistical comparison of all 20 flies measured before the training procedure resulted in a highly significant stronger correlation of 1-Oct and 3-Oct when compared to the correlation of 1-Oct and $\mathrm{MCH}$ and 3-Oct and $\mathrm{MCH}$, respectively (Figure 3.24 F). It can therefore be concluded that the similarity of 1 -Oct and 3-Oct is maintained in the neuronal activity pattern in the mushroom body.

\subsection{Learning to discriminate similar odorants}

It was investigated whether differential conditioning facilitates olfactory discrimination between similar odorants in comparison with absolute conditioning. It could be shown that differential conditioning indeed changed the ability of the flies to discriminate similar odorants. 1-octen-3-ol and 3-octanol are structurally very similar and strongly generalized by flies that were not trained differentially. Absolute training of one of the two odorants led to an avoidance of both odorants (Figure 3.7). Differential training, however, results in a discrimination of the odorants (Figure 3.10). It has to be mentioned that the pairing of 1-Oct with the punishment resulted in a stronger avoidance of the punished odorant in the subsequent test than 3-Oct. An explanation for this effect can be found in the activation pattern of the two odorants and will be discussed later. 


\section{Discussion}

Experiments in rats targeting similar questions as the present study led to comparable results. Absolute training of similar odorant mixtures resulted in a generalization of the two mixtures whereas differential conditioning increased the ability to discriminate between the two mixtures. This results was accompanied by the observation that the size of receptive field in the piriform cortex that get activated by the presentation of the mixture is decreased after differential training in contrast to absolute training after which the receptive field in increased [Chen et al., 2011]. The ability to discriminate between two similar odorants on a neuronal level depends on the separation of the activity patterns of neurons evoked by the odorant. Experiments in rats have shown that differential training of similar odorant mixtures increases the behavioral discrimination of the two mixtures and the activation pattern evoked by the olfactory stimuli were decorrelated in the piriform cortex. After explicitly training the animals not to discriminate between two distinct olfactory stimuli (by reinforcing both of them in contrast to a third stimulus), the animals generalized between the two mixtures and the activation pattern in piriform cortex showed a stronger correlation. Interestingly, this decorrelation could not be observed in the olfactory bulb [Chapuis \& Wilson, 2011].

A comparable study was also performed in humans. Subjects learned to discriminate two odorant enantiomers when one of them was paired with a negative reinforcer whereas a control enantiomer was not discriminated. Similarly, the correlation between the activity patterns evoked by the two enantiomers (measured by $\mathrm{fMRI}$ ) decreased after the differential conditioning whereas the correlation of the two forms of the control enantiomer stayed equally strong [Li et al., 2008].

\subsection{Olfactory discrimination in the antennal lobe}

The increase in discrimination ability of two similar odorants could be inhibited by blocking the synaptic output of inhibitory local interneurons type I (LN1) in the antennal lobe. Whereas the absolute conditioning of an odorant after negative reinforcement (Figure 3.16), the generalization of similar odorants after absolute conditioning (Figure 3.17) and the discrimination of dissimilar odorants after differential conditioning (Figure 3.18) was not affected, discrimination of similar odorants after differential conditioning was significantly decreased. LN1 neurons innervate 


\section{Discussion}

the cores of the glomeruli and do not arborize in the periphery (Figure 3.11B). As olfactory sensory neurons primarily innervate the periphery of a glomerulus Okada et al., 2009; (Tanaka et al., 2009], it can be assumed that LN1 neurons primarily form connections with projection neurons. A blockage of synaptic transmission of LN1 neurons is thereby disrupting the inhibition of PNs that might be responsible for a sharpening of the odor response in the projection neurons [Seki et al., 2010; Chou et al., 2010).

However, the recent study could not elucidate the question if LN1 activity is essential for the flies to learn the discrimination of similar odorants or if it is not possible for the flies to distinguish between the two stimuli in the first place. It is likely that blocking the synaptic transmission of GABAergic interneurons in the antennal lobe impairs the possibility of the flies to distinguish similar odorants. Previous experiments in honey bees suggest GABAergic neurons in the antennal lobe to be essential for discrimination of similar odorants [Stopfer et al., 1997]. This effect was confirmed by further experiments where the drug application to interrupt GABAergic signaling was timed to either memory consolidation or retrieval. In both cases, the discrimination between similar odorants was impaired [Hosler et al., 2000]. Additionally, an involvement of LN1 in the learning process would require a feedback from the mushroom body to the antennal lobes. Even though this feedback was suggested in Drosophila [Hu et al., 2010], no functional evidence could be found so far.

A functional involvement of local interneurons in the discrimination process can also be implied by the possibility to discriminate between two odorants with only one functional class of olfactory sensory neurons (that is responding to the two used odorants) [DasGupta \& Waddell, 2008] and the glomerular responses on the level of projection neurons without functional input to the glomerulus by OSNs [Shang et al. 2007]. It was proposed that an impairment in odor discrimination after the blocking of GABAergic inhibition in the antennal lobe is due to a desynchronisation of oscillatory responses in the projection neurons of bees [Stopfer et al., 1997]. However, this effect can be excluded in the present study as LN1 neurons were shown not to be involved in the generation of oscillatory responses to odors [Tanaka et al., 2009]. Additionally, the discrimination of two dissimilar odorants was not impaired in the present study (Figure 3.18). 


\section{Discussion}

The question whether the discrimination or the learned discrimination is impaired in the present study could be investigated by varying the time point of the blocking of synaptic transmission. Whereas synaptic transmission was blocked during the training and the test phase in the present study, it is possible to only block transmission during either training or testing. If an impaired synaptic transmission only during the test phase still results in a defect in discrimination, an effect of learning could be excluded. It is noteworthy that the copy number of the shibirets gene was gender specific in the present study. Female flies had one copy of the gene on the $x$-chromosome and one on the third chromosome whereas male flies only carried the gene on the third chromosome (see section B for the crossing schemes). However, this difference did not play a role during the experiments and no difference between male and female flies could be observed. This might be explained by the relatively long time the flies were kept at the restrictive temperature of $32{ }^{\circ} \mathrm{C}$ prior to the experiments. A single copy of the gene was thereby sufficient to block synaptic transmission. During an experiment with an activation of Shibirets only during the test and therefore a relative short time for the effect to occur, this difference might affect the results.

Unfortunately, our analysis of the expression patterns of type II inhibitory local interneurons (LN2) confirmed an expression of the GAL4-driver in motorneurons of the thoracic ganglion (see figure $3.12 \mathrm{~A}$ ). Therefore, a block of synaptic transmission with shibire ${ }^{\text {ts }}$ was not helpful due to the locomotion deficit. It would still be interesting to investigate the influence of LN2 neuron output during olfactory fine discrimination. That can only be achieved if the motorneurons in the thoracic ganglion are excluded from the targeting by GAL4. A possible way to ensure a specific expression only in the fly brain would be the use of GAL80 under the tsh-promotor [Shiga et al., 1996; Clyne \& Miesenböck, 2008]. By this means, an expression of the shibirets transgene in the thoracic ganglion would be inhibited by GAL80 while an expression in the central nervous system and thereby specifically in the type II inhibitory local interneurons would be feasible. 


\section{Discussion}

\subsection{Increased olfactory "acuity" as a result of differential training}

Association of a negative reinforcer with an olfactory stimulus (CS+) results in an avoidance of the odorant. After absolute training, the flies avoid the $\mathrm{CS}+$ when given the choice between the CS+ and a similar odorant. Differential training increases this avoidance significantly (Figure 3.20). This increase can be explained by an increased possibility to discriminate the two odorants after differential training and thereby an increased olfactory acuity.

Tully and Quinn proposed an effect of the CS- during differential condition in their original paper about classical olfactory conditioning in fruit flies [Tully \& Quinn, 1985]. Conversely, Masek and Heisenberg stated that the CS- was dispensable for associative learning at low concentrations [Masek \& Heisenberg, 2008]. However, only the effect of the CS- during learning of different odorant concentrations was tested. Additionally, it was impossible to compare the differences in the odorant concentrations used by Masek and Heisenberg and the odorant dilutions used in this study, as the air flow within the setup was higher in the previous study $(750 \mathrm{ml} / \mathrm{min}$ in comparison to $167 \mathrm{ml} / \mathrm{min}$ ) [Masek \& Heisenberg, 2008].

The acuity of the olfactory system was changed due to the differential training. However, it still remained unclear what is the underlying mechanism of the increased acuity. Previous studies have shown that differential associative memory consists of two traces. The excitatory trace is formed by the association of the conditioned stimulus with the reinforcement (CS+) whereas the inhibitory trace is formed by the absence of reinforcement during the presentation of the stimulus (CS-) [Rescorla, 1969a,b; Savastano et al., 1999]. It is not elucidated so far if the inhibitory trace (conditioned inhibition) plays a role in differential conditioning in the fly or if the conditioned excitation alone is responsible for the memory formation. Addressing this question is only possible with strongly generalized odors which were not used in olfactory associative learning in the fly until now.

Here, it could be shown that the presence of a CS- during the differential training leads to a conditioned inhibition of the CS- and therefore a decreased avoidance during the test against a novel odorant (Figure 3.22). It can be concluded that dif- 


\section{Discussion}

ferential training reduces the generalization of two similar odorants compared to absolute training as an effect of conditioned inhibition.

The investigation of the responses of the flies after differential training with a subsequent test of either the CS+ or the CS- against mineral oil resulted in an expected avoidance of the CS+. However, the CS- was not becoming a positive stimulus for the flies as they did not approach the odorant but still showed an avoidance behavior (Figure 3.23). This result can be explained by the fact that the discrimination of two stimuli is impeded if only one of the stimuli can be perceived [Lashley \& Wade, 1946]. The similarity between the two odorants seems to be stronger than the effect of the CS- as a conditioned inhibitor. Interestingly, the avoidance of 3-Oct as a CS- was significantly lower than the avoidance of 1-Oct as CS-. Therefore, 3-Oct seems to be a stronger conditioned inhibitor (and/or a weaker conditioned excitor) than 1-Oct. This asymmetry will be discussed in the next section.

\subsection{Asymmetric differential conditioning of 1-Oct and 3-Oct}

The increase of acuity of the olfactory system by differential training was further confirmed by testing the behavior of the flies to the CS+ and the CS- in a choice against mineral oil (Figure 3.23). After differential training, the CS+ was stronger avoided than after absolute training (Figure 3.7). Interestingly, the effect of differential training on the generalization of the two odorants depended on the odorant chosen as CS+. When 1-octen-3-ol was used as a CS+ during differential training, 3-octanol was avoided less than after absolute training. On the contrary, if 3-Oct was used as a CS+, 1-Oct was avoided stronger. The asymmetry was observed if both odorants are present in the choice test (Figure 3.10 and 3.20) and after differential training with a subsequent choice between mineral oil and the CS- (Figure 3.23). In all cases, the association of 1 -Oct with the electric shocks resulted in a lower preference index indicating a stronger learning than the association of 3-Oct with the punishment. An asymmetry in odor generalization was already observed in honey bees [Guerrieri et al., 2005]. Interestingly, no difference between the two odorants can be observed in the generalization or in any of the experiments where only one 


\section{Discussion}

of the similar odorants is present in the choice test. Neither the generalization after absolute training (Figures 3.7 and 3.22 ) nor the tests for the CS+ after differential training (Figure 3.23, striped graphs) showed differences between the odorants.

This asymmetry might be explained by the activity pattern of the olfactory sensory neurons in the antennal lobe obtained with calcium imaging. It could be demonstrated that 1-Oct and 3-Oct have very similar activity patterns compared to $\mathrm{MCH}$. However, the difference between the activity evoked by 1-Oct and 3-Oct lies in the activation of additional neurons by 1-Oct (see figure 3.5). These findings are in line with results obtained by Hallem \& Carlson [2006] as well as by Fishilevich \& Vosshall [2005]. A temporal pairing of 3-Oct with the electric shocks results in the association of the glomeruli activated by 3 -Oct with the punishment. As 1-Oct activates the same glomeruli, the choice between 1-Oct and 3-Oct is a choice between the activation of glomeruli associated with punishment and the activation of glomeruli activated with punishment and additional glomeruli. When, on the other hand, 1-Oct is associated with the electric shocks, all glomeruli activated by 1-Oct are associated with the punishment whereas all glomeruli activated by 3-Oct are associated with the absence of punishment. The flies can therefore associate the electric shocks with the activation of the glomeruli that are only activated by 1 -Oct and not by 3-Oct. Moreover, the flies learn that the activation of only a subset of the glomeruli associated with the electric shock is not sufficient to induce the punishment.

The described situation of 3-Oct being a subset of 1-Oct at the level of glomerular activation patterns makes it possible to discuss the asymmetry from another point of view. It can be seen as a problem of positive and negative patterning. During positive patterning a mixture of two stimuli $(A B)$ is reinforced whereas the compounds $A$ and $B$ are explicitly not reinforced $(A B+/ A-\& B-)$. Negative patterning on the other hand does not reinforce the mixture but the single compounds $(A B-/ A+\& B+)$. When the perception of 1 -Oct is considered as the sum of activated glomeruli, it can be understood as a mixture of activated glomeruli, AB. In this scenario, 3-Oct could be considered as a subset of these activated glomeruli, the compound $A$. The glomeruli activated only by 1-Oct are the second compound $B$. If 1-Oct is used as a CS+ and 3-Oct as a CS-, a positive patterning situation is created (AB+/A-). 3-Oct as the $\mathrm{CS}+$ and $1-$ Oct as a CS- represents a negative pattern problem (AB-/A+). Thereby, the choice of odorants results in two distinct conditioning paradigms. Interestingly, it 


\section{Discussion}

could be shown that Drosophila performs very well in positive patterning problems whereas a poor learning can be observed after negative patterning [Young et al., 2011; Wessnitzer et al., 2011]. This would explain the difference in the learning performance after the association of either 1-Oct or 3-Oct with the punishment.

\subsection{A possible localization of learned discrimination - A first attempt}

The localization of the neuronal correlate for the enhanced olfactory acuity can be implied as a localization of the acquisition of discriminative short-term memory. The present study could show that differential conditioning results in an increased discrimination of similar odorants. The mushroom body $\gamma$-lobe is implicated in the acquisition of short term memory after a single training phase. Input from dopaminergic cells via D1-like dopamine receptors (DopR) in the $\gamma$-lobe is crucial for memory acquisition [Qin et al., 2012]. Additionally, the $\alpha^{\prime} / \beta^{\prime}$-lobes have been implicated in memory acquisition and consolidation [Krashes et al., 2007] and a memory trace could be found [Wang et al., 2008]. The imaging of neuronal activity in the present study was performed in the horizontal lobes, focusing on the $\beta^{\prime}$ - and the $\gamma$-lobe. Besides a similarity of 1 -Oct and 3 -Oct before any conditioning, a possible effect of differential versus absolute training was investigated. Whereas absolute conditioning resulted in an even stronger correlation between 1-Oct and 3 -Oct in the $\beta^{\prime}$-lobe, a decrease in correlation could be found in the $\gamma$-shaft region after differential conditioning.

These results strikingly explain the generalization of similar odorants after absolute training and the discrimination after differential training. However, how can these results be explained on a systems or even cellular level? In order to find an answer to this question, intrinsic properties of the Kenyon cells were considered. It was previously shown that Kenyon cells have a sparse firing pattern with a small overlap between olfactory presentations. Interestingly, $\gamma$-neurons are having a higher firing threshold than $\beta$ '-neurons [Turner et al., 2008; Honegger et al., 2011]. In favor of this difference in the firing thresholds between $\gamma$ - and $\beta^{\prime}-K C$ speaks also the finding of the present study that the odor evoked calcium responses in the $\beta^{\prime}$-lobe are stronger in amplitude than in the $\gamma$-lobe (see figure $3.25 \mathrm{~B}$ and $\mathrm{C}$ ). 


\section{Discussion}

The sparse coding of the Kenyon cells implies very specific responses depending on the odorant presented. In the case of two similar odorants, it has to be assumed that an overlap between the KCs by the odorants exists. This is also confirmed by the finding that the activation patterns evoked by 1 -Oct and 3-Oct are strongly correlated before conditioning. As the firing threshold in the $\gamma$-neurons is higher than in the $\beta^{\prime}$-neurons, it is suggested that the $\beta^{\prime}$-neurons show a higher number of KCs with an overlapping response to 1 -Oct and 3 -Oct compared to the $\gamma$-lobe. In order

A

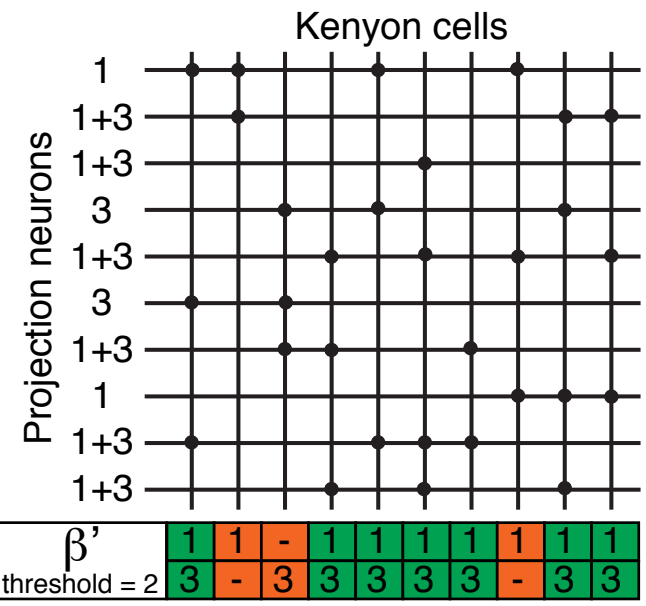

$\mathrm{B}$

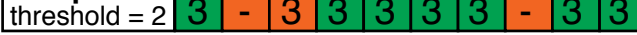

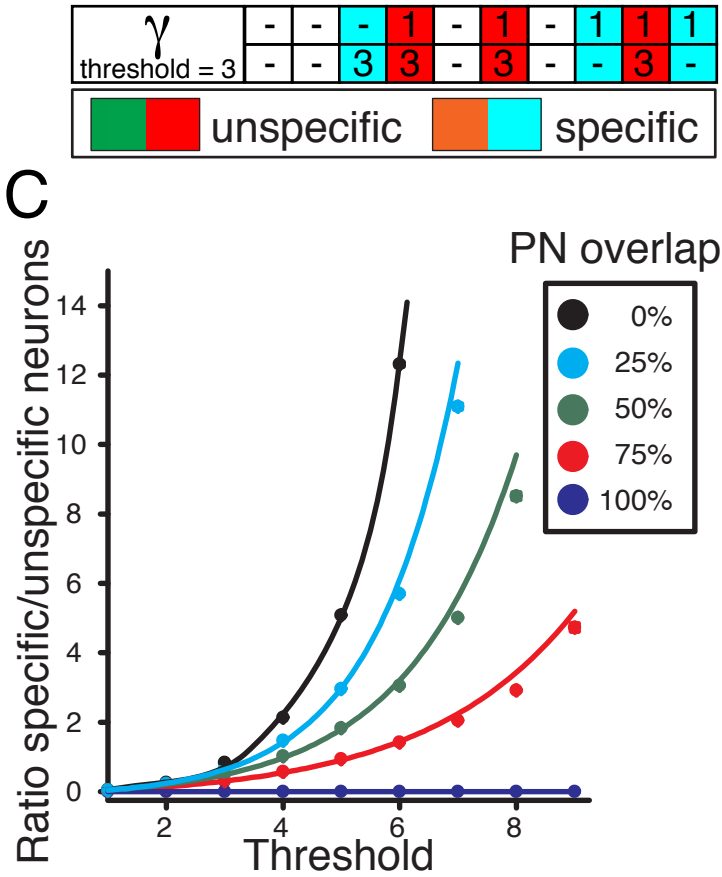

Figure 4.1. Connectivity model of similar odorants in the calyx.

A simple model of $P N$ input to the calyx was generated to verify the assumption that the reported higher firing threshold of $\gamma$-KCs results in an increased number of odor specific neurons. In contrast, the lower firing threshold of the $\beta$ ' -neurons suggest a higher density of neurons that respond to both of the similar odorants. (A) Example of a pattern with $10 \mathrm{PNs}$, $10 \mathrm{KCs}$ and a connectivity of 30 with a threshold of 2 for the $\beta$ '-lobe and 3 for the $\gamma$-lobe. Note that more PNs are conveying the information from both odorants. (B) Table of responses of the 10 depicted KCs in the example. The different threshold changes the responses. Specific neurons (response only to one of the odorants) are labeled in orange or magenta whereas unspecific neurons are colored green or red. (C) A more accurate model of the calyx includes 150 projection neurons, 2500 Kenyon cells and 33,450 connections as proposed by Turner et al. [2008]. 10,000 calculations of a random connectivity matrix showed that the ratio of specific:unspecific neurons is increasing exponentially with an increasing threshold. This holds true for a varying percentage of input overlap. 


\section{Discussion}

to test this hypothesis, a simple model was created. As depicted in figure $4.1 \mathrm{~A}$, the input to the mushroom body calyx is simplified by only taking neurons into account that respond to 1-Oct, 3-Oct or to both of them. The similarity of the response was simulated by considering $60 \%$ of the neurons to respond to both odorants, $20 \%$ specifically to 1 -Oct and $20 \%$ only to 3 -Oct. The strong simplification of the system implied the same connections between PNs and $\gamma-\mathrm{KC}$ and PNs and $\beta$ '-KCs with the same synaptic strength. The neuronal connections were randomly chosen. Figure $4.1 \mathrm{~B}$ shows the responsiveness of the $10 \mathrm{KCs}$. Whereas many neurons respond in the $\beta$ '-lobe , the number of cells responding in the $\gamma$-lobe is lower. Note that the $\beta$ '-neurons are responding much more unspecific.

This simple model of sparse encoding and differing thresholds confirmed the possibility that the $\beta$ '-lobe with a lower firing threshold contains more neurons that are responding to both odorants compared to the $\gamma$-lobe. In order to exclude any bias during the choosing of connections and to make the model more realistic, a computational model was generated. This model of the calyx includes 150 projection neurons, 2,500 Kenyon cells and 33,450 connections as predicted by Turner et al. 2008. Using these numbers, the computer was generating a random connectivity matrix and calculated the ratio of specific:unspecific neurons for varying thresholds. 10,000 subsequent calculations further ensured the randomness of the connections. In order to exclude the possibility that the number of overlapping neurons is crucial for this effect, the calculations were performed with a varying overlap. In all cases, 40 PNs were considered to be activated. Therefore, activity of 80 neurons was used as an input in the case of $0 \%$ similarity, only 70 neurons were considered in the case of $25 \%$ similarity and so on. All other PNs were ignored as they are not activated. The result of the calculations confirmed the assumption that a higher threshold increases the specificity of the neurons in this simplified model (Figure 4.1 C). Overall, the model confirmed that: I) the region with the lower threshold ( $\left.\beta^{\prime}\right)$ includes more neurons responding to stimulation. II) the number of neurons with an overlapping response to 1-Oct and 3-Oct is larger in the region with the lower threshold. III) even though less neurons respond to stimulation, the percentage of cells that react specifically to only one of the stimuli is larger in the region with the higher firing threshold $(\gamma)$. Despite the fact that this model is simplifying the connectivity in the mushroom body calyx very strongly, the 


\section{Discussion}

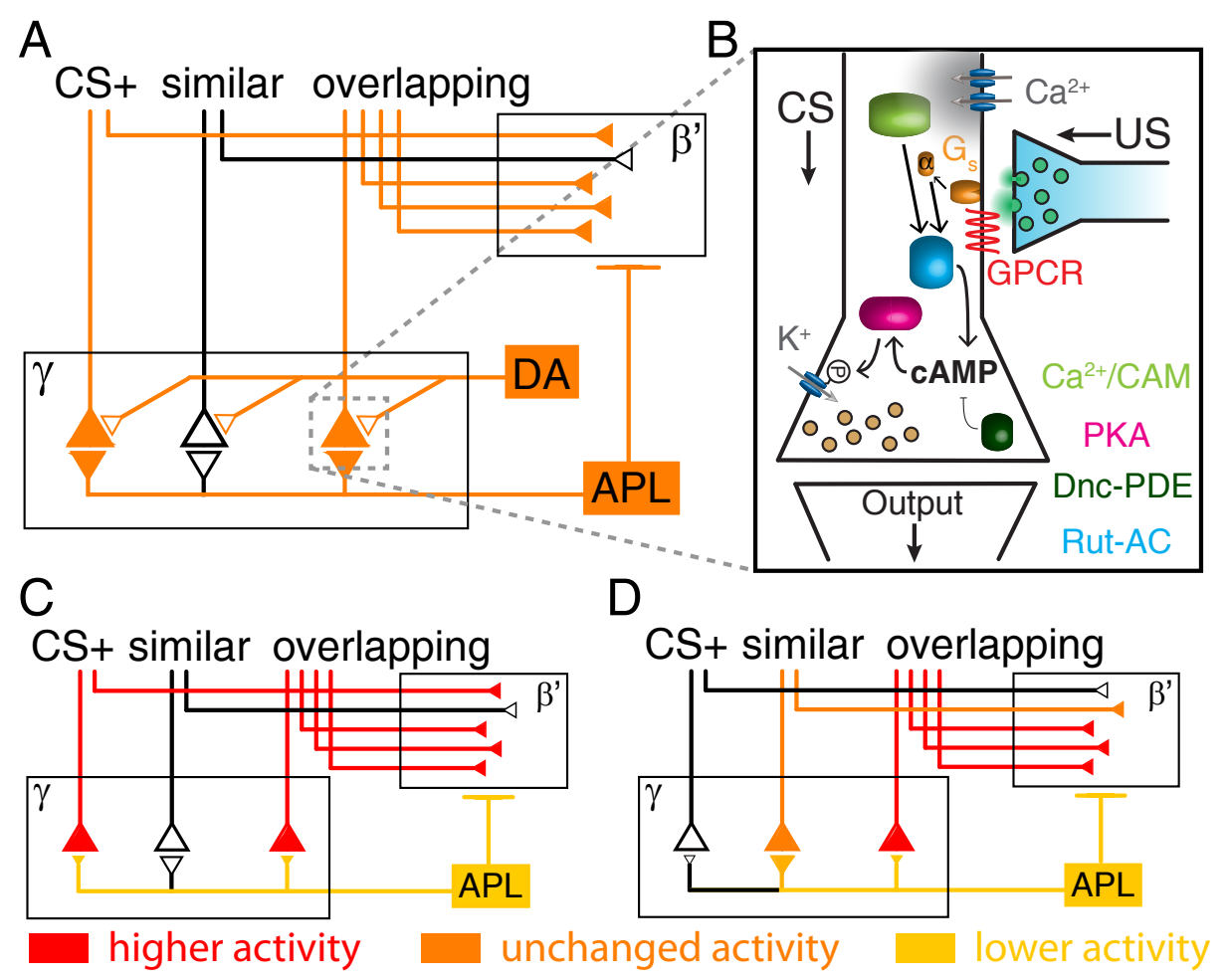

Figure 4.2. Hypothetical working model of cellular changes in response to absolute learning.

(A) During the training, the CS+ specific and the overlapping neurons are activated. Dopaminergic neurons are releasing DA on the synapses. Due to the coincidence detection, the cAMP levels in the neurons raise. An unknown mechanism weakens the synapses to the GABAergic APL neurons [Liu \& Davis, 2009]. (B) Scheme of the molecular changes in the KCs during coincidence detection. Simultaneous activation of calmodulin and the GPCR responding to dopamine results in a higher rutabaga-AC activity. In turn, the cAMP levels raise which triggers second messenger cascades. (C) The presentation of the CS+ after the training results in a higher activity of specific and unspecific neurons in the $\gamma$-shaft region due to the increased cAMP level. The weakened synapses from APL imply a weaker APL activity and thereby a weak inhibition of the $\beta$ '-lobe via the proposed feedback loop [Dubnau \& Chiang, 2013]. Hence, the activity in $\beta^{\prime}$ is increased. (D) Presentation of the similar odorant after the training activates the overlapping neurons in the $\gamma$-shaft as well as the specific neurons of the similar odorant. Whereas the weak synapses of the overlapping neurons weakly activate the APL neurons, the specific neurons show a normal activation. In summation, the resulting activation of APL is still weaker than before the training which results in an increased activity in the $\beta^{\prime}$-lobe. As this effect is elicited by both odorants, the correlation in $\beta^{\prime}$ increases. 


\section{Discussion}

assumption that a higher number of specific neurons can be found in the $\gamma$-lobe is not unlikely. On the other hand, a higher number of neurons responding to both odorants can be assumed to be found in the $\beta$ '-lobe.

A closer investigation of the mushroom body associated neurons involved in shortterm memory include dopaminergic neurons that relay the punishing information during the presentation of the CS+ to the $\gamma$-lobes [Qin et al., 2012]. APL neurons are GABAergic and innervate all mushroom body lobes and the calyx. They were shown to respond with a decreased activation to a presentation of the CS+ [Liu \& Davis, 2009. $\alpha^{\prime} / \beta^{\prime}-$ Kenyon cells respond with an increased activity to a CS+ Wang et al., 2008]. A functional feedback-loop from the mushroom body to the APL neurons and back is proposed [Dubnau \& Chiang, 2013]. It has also to be considered that the two odorants used as CS+ and CS- during this study are structurally similar and therefore induce similar activity patterns in the mushroom body as described above. Taking all these information into account, the following model is proposed.

During the presentation of the CS+ in the training phase, Kenyon cells in the $\gamma$-shaft region respond to the odorant stimulation with a depolarization and thereby an increase in intracellular calcium concentration. Additionally, dopaminergic neurons release dopamine and active G-protein coupled receptors in the KCs. The temporal coincidence of these two stimuli results in an increased cAMP level via the activation of the adenylat cyclase rutabaga. The high cAMP levels induce intracellular messenger pathways that increase PKA activity and in turn modulate potassium channel conductance and thereby increase the excitability of the neurons [Davis et al., 1995; Tomchik \& Davis, 2009; Gervasi et al., 2010] (Figure 4.2 B). Additionally, the connection to the APL neurons is weakend in a so far unknown process leading to a decreased APL activity in response to the odorant stimulation [Liu \& Davis, 2009]. If no CS- is presented during absolute training, an activation of the neurons responding to the CS+ is inducing a weaker activation of the APL neurons and thereby a decreased inhibition via GABA signaling (Figure $4.2 \mathrm{~A}$ ). If one assumes a connection from the APL neurons to the $\beta^{\prime}$-lobe, a lower GABA release would result in an decrease of inhibition of all KCs. Taken into consideration that the similar odorants are activating a largely overlapping number of KCs in the $\beta$ '-lobe, an increased activity of these overlapping neurons would result in a stronger correlation of the similar odorants upon CS+ and CS- stimulation after 


\section{Discussion}

the training. In the $\gamma$-shaft on the other hand, the CS+ specific neurons show an increased activation whereas the response of CS- specific neurons is not altered. This decorrelation is overshadowed by the overlapping neurons in the $\gamma$-shaft that are activated stronger upon odor presentation due to a higher CAMP level (Figure 4.2 C and D).

During differential training, the situation is changed as a CS- is presented. Whereas the effects described for the CS+ are of course overlapping, the presentation of the CS- changes the effects in all neurons that respond to the CS-. An activation of the KCs without the synchronous release of dopamine might lead to an effect similar to the one described for extinction: the cAMP levels in the responding neurons are decreased [Schwaerzel et al., 2002]. It is likely that the phosphodiestrase encoded by the dunce gene is involved in down regulating the cAMP level in KCs Gervasi et al. 2010]. In all KCs that are responding to the CS- and CS+, the increase of CAMP levels during the CS+ is neurons does not result in a weaker response from the APL neurons and the effect of an increased signal in the $\beta^{\prime}$-lobe is canceled out (Figure $4.3 \mathrm{~A}$ ). The calcium evoked responses in the $\beta^{\prime}$-lobe upon presentation of the CS+ are therefore similar to the responses before the training. Likewise, the CS- evoked activation pattern is not changed. The $\gamma$-shaft region, however, shows different activation patterns after the training. The cAMP levels in the CS+ specific neurons and thereby the PKA activity are increased which ultimately results in a higher calcium signal. Overlapping neurons on the other hand underwent an extinction-like process during the training [Schwaerzel et al., 2002] and therefore do not show an increased activity. CS- specific neurons were also subject to the extinction-like process and might have a decreased rate. These distinct responses of the CS+ and the CS- specific neurons can be an explanation for the observed decorrelation in the $\gamma$-shaft region of the mushroom body. As the CS+ specific neurons increase their activity and the CS- specific neurons might show a decrease or maximally unchanged response, the similarity of the odor evoked responses is decreasing. 


\section{Discussion}

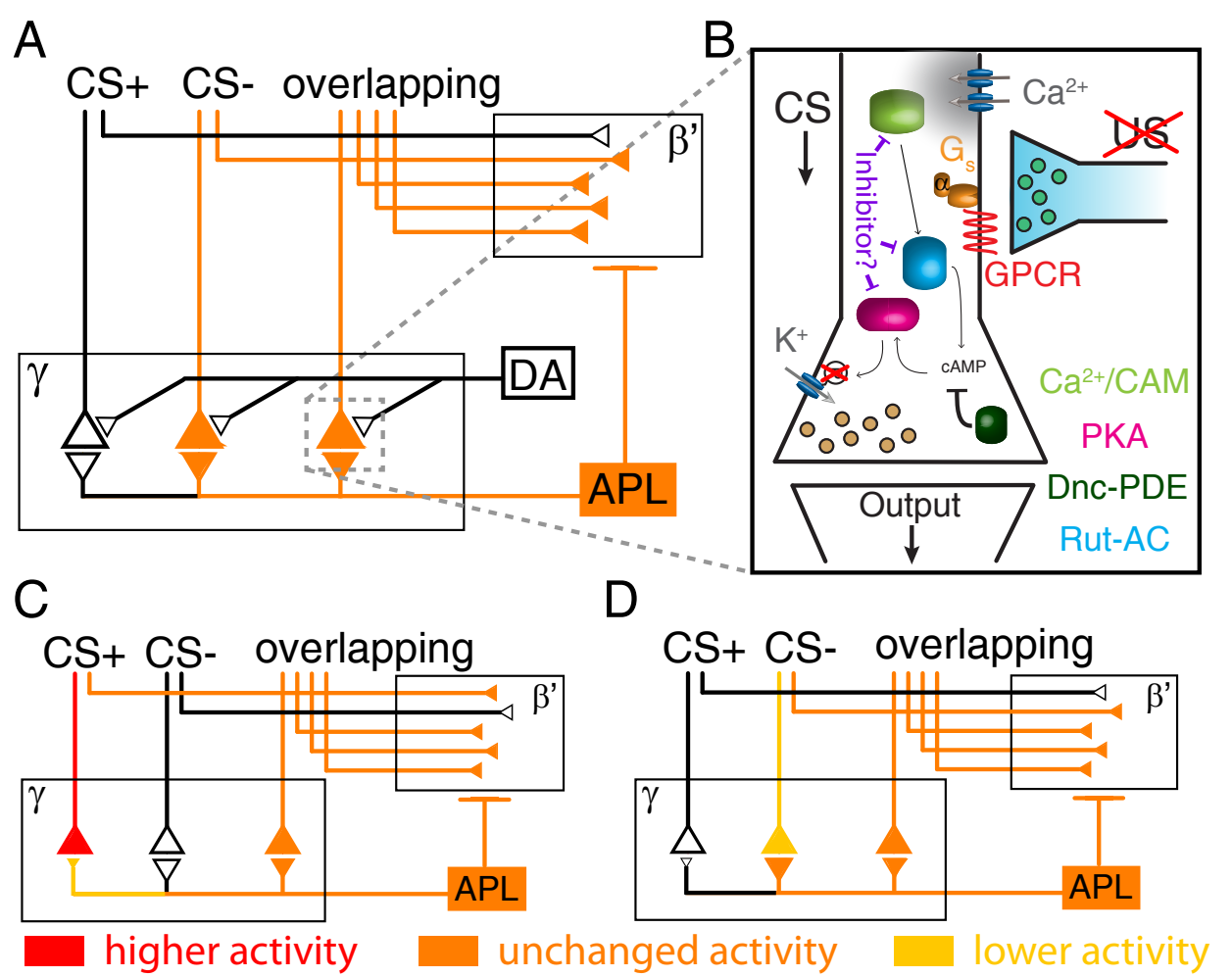

Figure 4.3. Hypothetical working model of cellular changes in response to differential learning.

The effects elicited by the CS+ are described on figure 4.2. Differential training additionally includes the CS-. (A) During the presentation of the CS-, the CS- specific and the overlapping neurons are activated. The lack of a simultaneous reinforcement signal elicits an extinction-like process in the overlapping neurons |Schwaerzel et al., 2002]. (B) Scheme of the molecular changes in the KCs during extinction. The absence of a reinforcing signal results in a decreased rutabaga-AC activity and thereby in a lower cAMP level and a countermanding effect of the increased excitability during the CS+. (C) The presentation of the CS+ after the training results in a higher activity of only CS+ specific neurons in the $\gamma$-shaft region. The unspecific neurons have a firing rate comparable to the situation before the training. APL activity is thereby also almost unchanged and the neurons in the $\beta$ '-lobe are inhibited. (D) Presentation of the CS- after the training activates the overlapping neurons in the $\gamma$-shaft as well as the CS- specific neurons. The overlapping neurons show an activity pattern as before the training and the specific neurons might respond weaker due to a lower cAMP level. Either way, the activity pattern between CS+ and CS- presentation are decorrelated as the specific CS+ neurons have a stronger activity. 


\section{Summary}

All animals are able to evaluate an odor by its consequences depending on prior experiences. This includes the possibility to show a resembling response to similar odors with the same consequence as well as a distinct response to similar odors with a different consequence. To accomplish these different tasks, the animal has to change its olfactory acuity, dependent on the relevance of olfactory stimuli and their consequences. Olfactory systems require mechanisms to combine and separate similar, yet slightly distinct patterns of neuronal activity. In the present study, it was investigated whether the olfactory acuity of the fruit fly Drosophila melanogaster could be altered depending on the situation. In particular, it was tried to decrease or increase the olfactory "acuity" by different conditioning paradigms.

To this means, two structurally similar odorants were identified. It was confirmed on a behavioral and physiological level that this chemical similarity was also conveyed to the flies nervous system. A generalization of the two odorants after absolute training of one of them showed a perceptual similarity. Activity measurements with calcium imaging in the antennal lobe and the mushroom body confirmed the similarity on a physiological level. In the antennal lobe, the glomerular activation pattern of the similar odorants was largely overlapping, whereas a dissimilar control odor showed a distinct pattern. The similarity in the mushroom body was proven by a stronger correlation between the similar odorants when compared to the correlations with the dissimilar control.

An enhancement of the olfactory acuity of the fly could be shown after differential training. After training the flies to discriminate between the two similar odorants by using one as a CS+ and the other as a CS- during differential conditioning, the similarity of the odorants was decreased. This was measured on a behavioral level by a choice test. Whereas absolute training showed a weak avoidance response of the punished odorant, the avoidance was substantially increased after differential train- 


\section{Summary}

ing. It could be shown that this increased avoidance can be ascribed to an effect of conditioned inhibition.

A closer look in the antennal lobe network, in particular the type I inhibitory local interneurons, could show the necessity of a functional inhibition in the antennal lobe during the olfactory discrimination. Without functional synaptic transmission of type I inhibitory local interneurons, a discrimination of the similar odorants after differential training was impossible. Interestingly, generalization of the similar odorants and discrimination of two dissimilar odorants was not impaired.

A neuronal correlate for the learned discrimination could be localized in the mushroom body horizontal lobes. Absolute training resulted in an even further increased similarity of the activation patterns evoked by two similar odorants in the $\beta$ '-lobe. Differential training on the contrary led to a decreased similarity of the activation patterns in the $\gamma$-shaft region of the $\gamma$-lobe.

It can be concluded that an enhancement of the olfactory acuity in Drosophila is possible when the animals are explicitly trained to discriminate between two similar odorants. In order to fulfill this task, local interneurons in the antennal lobe play a crucial role. Neuronal properties in the mushroom body are changing during the differential conditioning process so that the activation pattern of previously similar odorants become more dissimilar. 


\section{Bibliography}

Abràmoff MD, Magalhães PJ \& Ram SJ (2004), Image processing with ImageJ., Biophotonics international, 11(7):36-42.

Aceves-Piña EO \& Quinn WG (1979), Learning in normal and mutant Drosophila larvae., Science, 206(4414):93-96.

Ache BW \& Young JM (2005), Olfaction: Diverse Species, Conserved Principles., Neuron, 48(3):417-430.

Adams MD, Celniker SE, Holt RA, Evans CA, Gocayne JD, Amanatides PG, Scherer SE, Li PW, Hoskins RA, Galle RF, George RA, Lewis SE, Richards S, Ashburner M, Henderson SN, Sutton GG, Wortman JR, Yandell MD, Zhang Q, Chen LX, Brandon RC, Rogers YH, Blazej RG, Champe M, Pfeiffer BD, Wan KH, Doyle C, Baxter EG, Helt G, Nelson CR, Gabor GL, Abril JF, Agbayani A, An HJ, Andrews-Pfannkoch C, Baldwin D, Ballew RM, Basu A, Baxendale J, Bayraktaroglu L, Beasley EM, Beeson KY, Benos PV, Berman BP, Bhandari D, Bolshakov S, Borkova D, Botchan MR, Bouck J, Brokstein P, Brottier P, Burtis KC, Busam DA, Butler H, Cadieu E, Center A, Chandra I, Cherry JM, Cawley S, Dahlke C, Davenport LB, Davies P, de Pablos B, Delcher A, Deng Z, Mays AD, Dew I, Dietz SM, Dodson K, Doup LE, Downes M, DuganRocha S, Dunkov BC, Dunn P, Durbin KJ, Evangelista CC, Ferraz C, Ferriera S, Fleischmann W, Fosler C, Gabrielian AE, Garg NS, Gelbart WM, Glasser K, Glodek A, Gong F, Gorrell JH, Gu Z, Guan P, Harris M, Harris NL, Harvey D, Heiman TJ, Hernandez JR, Houck J, Hostin D, Houston KA, Howland TJ, Wei MH, Ibegwam C, Jalali M, Kalush F, Karpen GH, Ke Z, Kennison JA, Ketchum KA, Kimmel BE, Kodira CD, Kraft C, Kravitz S, Kulp D, Lai Z, Lasko P, Lei Y, Levitsky AA, Li J, Li Z, Liang Y, Lin X, Liu X, Mattei B, Mclntosh TC, McLeod MP, McPherson D, Merkulov G, Milshina NV, Mobarry C, Morris J, Moshrefi 
A, Mount SM, Moy M, Murphy B, Murphy L, Muzny DM, Nelson DL, Nelson DR, Nelson KA, Nixon K, Nusskern DR, Pacleb JM, Palazzolo M, Pittman GS, Pan S, Pollard J, Puri V, Reese MG, Reinert K, Remington K, Saunders RD, Scheeler F, Shen H, Shue BC, Sidén-Kiamos I, Simpson M, Skupski MP, Smith T, Spier E, Spradling AC, Stapleton M, Strong R, Sun E, Svirskas R, Tector C, Turner R, Venter E, Wang AH, Wang X, Wang ZY, Wassarman DA, Weinstock GM, Weissenbach J, Williams SM, WoodageT, Worley KC, Wu D, Yang S, Yao QA, Ye J, Yeh RF, Zaveri JS, Zhan M, Zhang G, Zhao Q, Zheng L, Zheng XH, Zhong FN, Zhong W, Zhou X, Zhu S, Zhu X, Smith HO, Gibbs RA, Myers EW, Rubin GM \& Venter JC (2000), The genome sequence of Drosophila melanogaster., Science, 287(5461):2185-2195.

Akalal DBG, Wilson CF, Zong L, Tanaka NK, Ito K \& Davis RL (2006), Roles for Drosophila mushroom body neurons in olfactory learning and memory., Learning \& Memory, 13(5):659-668.

Akalal DBG, Yu D \& Davis RL (2010), A Late-Phase, Long-Term Memory Trace Forms in the $\gamma$ Neurons of Drosophila Mushroom Bodies after Olfactory Classical Conditioning., Journal of Neuroscience, 30(49):16699-16708.

Akalal DBG, Yu D \& Davis RL (2011), The Long-Term Memory Trace Formed in the Drosophila $\alpha / \beta$ Mushroom Body Neurons Is Abolished in Long-Term Memory Mutants., Journal of Neuroscience, 31(15):5643-5647.

Armstrong JD, Kaiser K, Müller A, Fischbach KF, Merchant N \& Strausfeld NJ (1995), Flybrain, an on-line atlas and database of the Drosophila nervous system., Neuron, 15(1):17-20.

Aso Y, Grübel K, Busch S, Friedrich AB, Siwanowicz I \& Tanimoto H (2009), The Mushroom Body of Adult Drosophila Characterized by GAL4 Drivers., Journal of Neurogenetics, 23(1-2):156-172.

Aso Y, Herb A, Ogueta M, Siwanowicz I, Templier T, Friedrich AB, Ito K, Scholz H \& Tanimoto H (2012), Three Dopamine Pathways Induce Aversive Odor Memories with Different Stability., PLoS Genetics, 8(7):e1002768. 


\section{Bibliography}

Aso Y, Siwanowicz I, Bräcker L, Ito K, Kitamoto T \& Tanimoto H (2010), Specific Dopaminergic Neurons for the Formation of Labile Aversive Memory., Current Biology, 20(16):1445-1451.

Baek M \& Mann RS (2009), Lineage and Birth Date Specify Motor Neuron Targeting and Dendritic Architecture in Adult Drosophila., Journal of Neuroscience, 29(21):6904-6916.

Baines RA, Uhler JP, Thompson A, Sweeney ST \& Bate M (2001), Altered electrical properties in Drosophila neurons developing without synaptic transmission., Journal of Neuroscience, 21(5):1523-1531.

Baird GS, Zacharias DA \& Tsien RY (1999), Circular permutation and receptor insertion within green fluorescent proteins., Proceedings of the National Academy of Sciences, 96(20):11241-11246.

Baubet V, Le Mouellic H, Campbell AK, Lucas-Meunier E, Fossier P \& Brûlet P (2000), Chimeric green fluorescent protein-aequorin as bioluminescent $\mathrm{Ca}^{2+}$ reporters at the single-cell level, Proceedings of the National Academy of Sciences, 97(13):7260-7265.

Beck CD, Schroeder B \& Davis RL (2000), Learning performance of normal and mutant Drosophila after repeated conditioning trials with discrete stimuli., Journal of Neuroscience, 20(8):2944-2953.

Benton R, Sachse S, Michnick SW \& Vosshall LB (2006), Atypical Membrane Topology and Heteromeric Function of Drosophila Odorant Receptors In Vivo., PLoS Biol, 4(2):e20.

Benton R, Vannice KS, Gomez-Diaz C \& Vosshall LB (2009), Variant Ionotropic Glutamate Receptors as Chemosensory Receptors in Drosophila., Cell, 136(1):149-162.

Berridge MJ (1998), Neuronal calcium signaling., Neuron, 21(1):I3-26.

Bhandawat V, Olsen SR, Gouwens NW, Schlief ML \& Wilson RI (2007), Sensory processing in the Drosophila antennal lobe increases reliability and separability of ensemble odor representations., Nature Neuroscience, 10(11):1474-1482. 


\section{Bibliography}

Blum A \& Dubnau JT (2010), Parallel processing of olfactory memories in Drosophila., Fly, 4(2):163-166.

Boyden ES, Zhang F, Bamberg E, Nagel G \& Deisseroth K (2005), Millisecondtimescale, genetically targeted optical control of neural activity., Nature Neuroscience, 8(9):1263-1268.

Brand AH \& Perrimon N (1993), Targeted gene expression as a means of altering cell fates and generating dominant phenotypes., Development, 118(2):401-415.

Brembs B \& Heisenberg M (2001), Conditioning with compound stimuli in Drosophila melanogaster in the flight simulator., The Journal of Experimental Biology, 204:2849-2859.

Brogden WJ (1939), Sensory pre-conditioning., Journal of Experimental Psychology, 25(4):323-332.

Buck L \& Axel R (1991), A novel multigene family may encode odorant receptors: a molecular basis for odor recognition., Cell, 65(1):175-187.

Busto GU, Cervantes-Sandoval I \& Davis RL (2010), Olfactory Learning in Drosophila., Physiology, 25(6):338-346.

Butcher NJ, Friedrich AB, Lu Z, Tanimoto H \& Meinertzhagen IA (2012), Different classes of input and output neurons reveal new features in microglomeruli of the adult Drosophila mushroom body calyx., The Journal of Comparative Neurology, 520(10):2185-2201.

Cervantes-Sandoval I \& Davis RL (2012), Distinct Traces for Appetitive versus Aversive Olfactory Memories in DPM Neurons of Drosophila., Current Biology, 22(13):1247-1252.

Chabaud MA, Devaud JM, Pham-Delègue MH, Preat T \& Kaiser L (2006), OIfactory conditioning of proboscis activity in Drosophila melanogaster., Journal of Comparative Physiology A, 192(12):1335-1348.

Chapuis J \& Wilson DA (2011), Bidirectional plasticity of cortical pattern recognition and behavioral sensory acuity., Nature Publishing Group, 15(1):155-161. 
Chen CC, Wu JK, Lin HW, Pai TP, Fu TF, Wu CL, Tully T \& Chiang AS (2012), Visualizing long-term memory formation in two neurons of the Drosophila brain., Science, 335(6069):678-685.

Chen CFF, Barnes DC \& Wilson DA (2011), Generalized vs. stimulus-specific learned fear differentially modifies stimulus encoding in primary sensory cortex of awake rats., Journal of Neurophysiology, 106(6):3136-3144.

Chen MS, Obar RA, Schroeder CC, Austin TW, Poodry CA, Wadsworth SC \& Vallee RB (1991), Multiple forms of dynamin are encoded by shibire, a Drosophila gene involved in endocytosis., Nature, 351(6327):583-586.

Chou YH, Spletter ML, Yaksi E, Leong JCS, Wilson RI \& Luo L (2010), Diversity and wiring variability of olfactory local interneurons in the Drosophila antennal lobe., Nature Publishing Group, 13(4):439-449.

Claridge-Chang A, Roorda RD, Vrontou E, Sjulson L, Li H, Hirsh J \& Miesenböck G (2009), Writing Memories with Light-Addressable Reinforcement Circuitry., Cell, 139(2):405-415.

Cleland TA, Morse A, Yue EL \& Linster C (2002), Behavioral models of odor similarity., Behavioral Neuroscience, 116(2):222-231.

Cleland TA \& Narla VA (2003), Intensity Modulation of Olfactory Acuity., Behavioral Neuroscience, 117(6):1434-1440.

Clyne JD \& Miesenböck G (2008), Sex-Specific Control and Tuning of the Pattern Generator for Courtship Song in Drosophila., Cell, 133(2):354 - 363.

Clyne PJ, Warr CG, Freeman MR, Lessing D, Kim J \& Carlson JR (1999), A Novel Family of Divergent Seven-Transmembrane Proteins: Candidate Odorant Receptors in Drosophila., Neuron, 22(2):327 - 338.

Connolly JB, Roberts IJH, Armstrong JD, Kaiser K, Forte M, Tully T \& OḰane CJ (1996), Associative Learning Disrupted by Impaired $\mathrm{G}_{\mathrm{s}}$ Signaling in Drosophila Mushroom Bodies., Science, 274(5295):2104-2107. 


\section{Bibliography}

Coureaud G, Hamdani Y, Schaal B \& Thomas-Danguin T (2009), Elemental and configural processing of odour mixtures in the newborn rabbit., Journal of Experimental Biology, 212(16):2525-2531.

Couto A, Alenius M \& Dickson BJ (2005), Molecular, Anatomical, and Functional Organization of the Drosophila Olfactory System., Current Biology, 15(17):15351547.

Crittenden JR, Skoulakis EM, Han KA, Kalderon D \& Davis RL (1998), Tripartite mushroom body architecture revealed by antigenic markers., Learning \& Memory, $5(1): 38-51$.

Das S, Sadanandappa MK, Dervan A, Larkin A, Lee JA, Sudhakaran IP, Priya R, Heidari R, Holohan EE \& Pimentel A (2011), Plasticity of local GABAergic interneurons drives olfactory habituation., Proceedings of the National Academy of Sciences, 108(36):E646-E654.

DasGupta S \& Waddell S (2008), Learned Odor Discrimination in Drosophila without Combinatorial Odor Maps in the Antennal Lobe., Current Biology, 18(21):1668-1674.

Davis RL (2004), Olfactory Learning., Neuron, 44(1):31-48.

Davis RL (2011), Traces of Drosophila Memory., Neuron, 70(1):8-19.

Davis RL, Cherry J, Dauwalder B, Han PL \& Skoulakis E (1995), The cyclic AMP system and Drosophila learning., Molecular and cellular biochemistry, 149150:271-278.

de Belle JS \& Heisenberg M (1994), Associative odor learning in Drosophila abolished by chemical ablation of mushroom bodies., Science, 263(5147):692-694.

de Bruyne M, Foster K \& Carlson JR (2001), Odor coding in the Drosophila antenna., Neuron, 30(2):537-552.

Deisig N (2003), A Modified Version of the Unique Cue Theory Accounts for Olfactory Compound Processing in Honeybees., Learning \& Memory, 10(3):199-208. 
Deisig N, Giurfa M, Lachnit H \& Sandoz JC (2006), Neural representation of olfactory mixtures in the honeybee antennal lobe., European Journal of Neuroscience, 24(4):1161-1174.

Diegelmann S, Bate M \& Landgraf M (2008), Gateway cloning vectors for the LexAbased binary expression system in Drosophila., Fly, 2(4):236-239.

Dill M, Wolf R \& Heisenberg M (1993), Visual pattern recognition in Drosophila involves retinotopic matching., Nature, 365:751-753.

Dobritsa AA, van der Goes van Naters W, Warr CG, Steinbrecht RA \& Carlson JR (2003), Integrating the Molecular and Cellular Basis of Odor Coding in the Drosophila Antenna., Neuron, 37(5):827-841.

Dubnau J \& Chiang AS (2013), Systems memory consolidation in Drosophila., Current Opinion in Neurobiology, 23(1):84-91.

Dubnau J, Chiang AS, Grady L, Barditch J, Gossweiler S, McNeil J, Smith P, Buldoc F, Scott R, Certa U et al. (2003), The staufen/pumilio Pathway Is Involved in Drosophila Long-Term Memory, Current Biology, 13(4):286-296.

Dubnau J, Grady L, Kitamoto T \& Tully T (2001), Disruption of neurotransmission in Drosophila mushroom body blocks retrieval but not acquisition of memory., Nature, 411(6836):476-480.

Dudai Y, Jan YN, Byers D, Quinn WG \& Benzer S (1976), dunce, a mutant of Drosophila deficient in learning., Proceedings of the National Academy of Sciences of the United States of America, 73(5):1684-1688.

Duffy JB (2002), GAL4 system in Drosophila: A fly geneticist's swiss army knife., genesis, 34(1-2):1-15.

Elmore T, Ignell R, Carlson JR \& Smith DP (2003), Targeted mutation of a Drosophila odor receptor defines receptor requirement in a novel class of sensillum., The Journal of neuroscience, 23(30):9906-9912.

Erber J, Masuhr T \& Menzel R (1980), Localization of short-term memory in the brain of the bee, Apis mellifera., Physiological Entomology, 5(4):343-358. 


\section{Bibliography}

Faber T, Joerges J \& Menzel R (1999), Associative learning modifies neural representations of odors in the insect brain., Nature Neuroscience, 2:74-78.

Fasshauer D, Sutton RB, Brunger AT \& Jahn R (1998), Conserved structural features of the synaptic fusion complex: SNARE proteins reclassified as Q- and R-SNAREs., Proceedings of the National Academy of Sciences, 95(26):1578115786.

Feany M \& Quinn W (1995), A neuropeptide gene defined by the Drosophila memory mutant amnesiac, Science, 268(5212):869-873.

Feany MB \& Bender WW (2000), A Drosophila model of Parkinson's disease., Nature, 404(6776):394-398.

Fiala A (2007), Olfaction and olfactory learning in Drosophila: recent progress., Current Opinion in Neurobiology, 17(6):720-726.

Fiala A, Spall T, Diegelmann S, Eisermann B, Sachse S, Devaud JM, Buchner E \& Galizia CG (2002), Genetically expressed cameleon in Drosophila melanogaster is used to visualize olfactory information in projection neurons., Current biology, 12(21):1877-1884.

Fiala A, Suska A \& Schlüter OM (2010), Optogenetic Approaches in Neuroscience., Current Biology, 20(20):R897 - R903.

Fishilevich E \& Vosshall LB (2005), Genetic and Functional Subdivision of the Drosophila Antennal Lobe., Current Biology, 15(17):1548-1553.

Fletcher ML \& Wilson DA (2002), Experience modifies olfactory acuity: acetylcholine-dependent learning decreases behavioral generalization between similar odorants., Journal of Neuroscience, 22(2):RC201.

Friedrich RW (2006), Mechanisms of odor discrimination: neurophysiological and behavioral approaches., Trends in Neurosciences, 29(1):40 - 47.

Friedrich RW \& Korsching SI (1997), Combinatorial and Chemotopic Odorant Coding in the Zebrafish Olfactory Bulb Visualized by Optical Imaging., Neuron, 18(5):737 - 752 . 


\section{Bibliography}

Friggi-Grelin F, Coulom HIn, Meller M, Gomez D, Hirsh J \& Birman S (2003), Targeted gene expression in Drosophila dopaminergic cells using regulatory sequences from tyrosine hydroxylase., Journal of neurobiology, 54(4):618-627.

Galizia CG, Munch D, Strauch M, Nissler A \& Ma S (2010), Integrating Heterogeneous Odor Response Data into a Common Response Model: A DoOR to the Complete Olfactome., Chemical Senses, 35(7):551-563.

Galizia CG, Sachse S, Rappert A \& Menzel R (1999), The glomerular code for odor representation is species specific in the honeybee Apis mellifera., Nature Neuroscience, 2:473-478.

Galizia CG \& Szyszka P (2008), Olfactory coding in the insect brain: molecular receptive ranges, spatial and temporal coding., Entomologia Experimentalis et Applicata, 128(1):81-92.

Ganetzky B \& Flanagan JR (1978), On the relationship between senescence and age-related changes in two wild-type strains of Drosophila melanogaster., Experimental gerontology, 13(3):189-196.

Gao Q \& Chess A (1999), Identification of Candidate Drosophila Olfactory Receptors from Genomic DNA Sequence., Genomics, 60(1):31 - 39.

Gao Q, Yuan B \& Chess A (2000), Convergent projections of Drosophila olfactory neurons to specific glomeruli in the antennal lobe., Nature neuroscience, 3(8):780-785.

Gerber B \& Stocker RF (2007), The Drosophila larva as a model for studying chemosensation and chemosensory learning: a review., Chemical senses, 32(1):65-89.

Gerber B, Tanimoto H \& Heisenberg M (2004), An engram found? Evaluating the evidence from fruit flies., Current opinion in neurobiology, 14(6):737-744.

Gervasi N, Tchénio P \& Preat T (2010), PKA Dynamics in a Drosophila Learning Center: Coincidence Detection by Rutabaga Adenylyl Cyclase and Spatial Regulation by Dunce Phosphodiesterase., Neuron, 65(4):516-529. 


\section{Bibliography}

Girardin CC, KreissI S \& Galizia CG (2013), Inhibitory connections in the honeybee antennal lobe are spatially patchy., Journal of Neurophysiology, 109(2):332-343.

Giurfa M (2004), Conditioning procedure and color discrimination in the honeybee Apis mellifera., Naturwissenschaften, 91(5):228-231.

Giurfa M (2007), Behavioral and neural analysis of associative learning in the honeybee: a taste from the magic well., Journal of comparative physiology. A, Sensory, neural, and behavioral physiology, 193(8):801-824.

Goldman AL, Van der Goes van Naters W, Lessing D, Warr CG \& Carlson JR (2005), Coexpression of two functional odor receptors in one neuron., Neuron, 45(5):661-666.

Goodwin SF, Del Vecchio M, Velinzon K, Hogel C, Russell SR, Tully T \& Kaiser K (1997), Defective learning in mutants of the Drosophila gene for a regulatory subunit of cAMP-dependent protein kinase., The Journal of neuroscience, 17(22):8817-8827.

Greenspan RJ (2004), Fly Pushing, The Theory and Practice of Drosophila Genetics, CSHL Press, 2 edition, ISBN 9780879697112.

Griesbeck O (2004), Fluorescent proteins as sensors for cellular functions., Current Opinion in Neurobiology, 14(5):636-641.

Groh C, Ahrens D \& Rössler W (2006), Environment-and age-dependent plasticity of synaptic complexes in the mushroom bodies of honeybee queens., Brain, behavior and evolution, 68(1):1-14.

Groh C \& Rössler W (2011), Comparison of microglomerular structures in the mushroom body calyx of neopteran insects., Arthropod structure \& development, 40(4):358-367.

Guerrero G \& Isacoff EY (2001), Genetically encoded optical sensors of neuronal activity and cellular function., Current Opinion in Neurobiology, 11(5):601-607.

Guerrieri F, Schubert M, Sandoz JC \& Giurfa M (2005), Perceptual and Neural Olfactory Similarity in Honeybees., PLoS Biology, 3(4):e60. 


\section{Bibliography}

Guizar-Sicairos M, Thurman ST \& Fienup JR (2008), Efficient subpixel image registration algorithms., Optics letters, 33(2):156-158.

Guo J \& Guo A (2005), Crossmodal Interactions Between Olfactory and Visual Learning in Drosophila., Science, 309(5732):307-310.

Guttman N \& Kalish HI (1956), Discriminability and stimulus generalization., Journal of Experimental Psychology.

Haddad R, Khan R, Takahashi YK, Mori K, Harel D \& Sobel N (2008), A metric for odorant comparison., Nature Methods, 5(5):425-429.

Haehnel M \& Menzel R (2012), Long-term memory and response generalization in mushroom body extrinsic neurons in the honeybee Apis mellifera., Journal of Experimental Biology, 215(3):559-565.

Hallem EA \& Carlson JR (2006), Coding of Odors by a Receptor Repertoire., Cell, 125(1):143-160.

Hallem EA, Ho MG \& Carlson JR (2004), The molecular basis of odor coding in the Drosophila antenna., Cell, 117(7):965-979.

Hamada FN, Rosenzweig M, Kang K, Pulver SR, Ghezzi A, Jegla TJ \& Garrity PA (2008), An internal thermal sensor controlling temperature preference in Drosophila., Nature, 454(7201):217-220.

Han KA, Millar NS \& Davis RL (1998), A Novel Octopamine Receptor with Preferential Expression in Drosophila Mushroom Bodies., The Journal of neuroscience, 18(10):3650-3658.

Han KA, Millar NS, Grotewiel MS \& Davis RL (1996), DAMB, a novel dopamine receptor expressed specifically in Drosophila mushroom bodies., $\mathrm{Neu}$ ron, 16(6):1127-1135.

Hartley JL, Temple GF \& Brasch MA (2000), DNA cloning using in vitro sitespecific recombination., Genome Research, 10(11):1788-1795. 


\section{Bibliography}

Hazelrigg T, Levis R \& Rubin GM (1984), Transformation of white locus DNA in drosophila: dosage compensation, zeste interaction, and position effects., Cell, 36(2):469-481.

Hearst E \& Franklin SR (1977), Positive and negative relations between a signal and food: Approach-withdrawal behavior to the signal., Journal of experimental psychology. Animal behavior processes, 3(1):37.

Heim N \& Griesbeck O (2004), Genetically encoded indicators of cellular calcium dynamics based on troponin $\mathrm{C}$ and green fluorescent protein., Journal of biological chemistry, 279(14):14280-14286.

Heisenberg M (2003), Mushroom body memoir: from maps to models., Nature Reviews Neuroscience, 4(4):266-275.

Heisenberg M, Borst A, Wagner S \& Byers D (1985), Drosophila Mushroom Body Mutants are Deficient in Olfactory Learning., Journal of Neurogenetics, 2(1):1-30.

Henley JR, Cao H \& McNiven MA (1999), Participation of dynamin in the biogenesis of cytoplasmic vesicles., FASEB journal : official publication of the Federation of American Societies for Experimental Biology, 13 Suppl 2:S243-7.

Hermann M (2011), Olfactory discrimination learning in Drosophila., Bachelor thesis, Georg-August University Göttingen.

Hodge JJ (2009), Ion channels to inactivate neurons in Drosophila., Frontiers in Molecular Neuroscience, 2:13.

Hofbauer A (1991), Eine Bibliothek monoklonaler Antikörper gegen das Gehirn von Drosophila melanogaster., Ph.D. thesis, Universität Würzburg.

Honegger KS, Campbell RAA \& Turner GC (2011), Cellular-Resolution Population Imaging Reveals Robust Sparse Coding in the Drosophila Mushroom Body., Journal of Neuroscience, 31(33):11772-11785.

Honig WK \& Urcuioli PJ (1981), The legacy of Guttman and Kalish (1956): Twentyfive years of research on stimulus generalization., Journal of the Experimental Analysis of Behavior, 36(3):405. 


\section{Bibliography}

Hosler JS, Buxton KL \& Smith BH (2000), Impairment of olfactory discrimination by blockade of GABA and nitric oxide activity in the honey bee antennal lobes., Behavioral Neuroscience; Behavioral Neuroscience, 114(3):514.

Hu A, Zhang W \& Wang Z (2010), Functional feedback from mushroom bodies to antennal lobes in the Drosophila olfactory pathway., Proceedings of the National Academy of Sciences of the United States of America, 107(22):10262-10267.

Huang J, Zhang W, Qiao W, Hu A \& Wang Z (2010), Functional Connectivity and Selective Odor Responses of Excitatory Local Interneurons in Drosophila Antennal Lobe., Neuron, 67(6):1021-1033.

Ito K, Suzuki K, Estes P, Ramaswami M, Yamamoto D \& Strausfeld NJ (1998), The organization of extrinsic neurons and their implications in the functional roles of the mushroom bodies in Drosophila melanogaster Meigen, Learning \& Memory, 5(1):52-77.

Jefferis GS, Marin EC, Stocker RF \& Luo L (2001), Target neuron prespecification in the olfactory map of Drosophila., Nature, 414(6860):204-208.

Josens R, Eschbach C \& Giurfa M (2009), Differential conditioning and long-term olfactory memory in individual Camponotus fellah ants., Journal of Experimental Biology, 212(12):1904-1911.

Kalman RE (1960), A new approach to linear filtering and prediction problems., Journal of basic Engineering, 82(1):35-45.

Kandel ER, Jessell TM \& Schwartz JH (2000), Principles of neural science, McGraw-Hill, 4th edition edition.

Kasuya $\mathbf{J}$ (2009), Neuronal mechanisms of learning and memory revealed by spatial and temporal suppression of neurotransmission using shibire ${ }^{t s 1}$, a temperaturesensitive dynamin mutant gene in Drosophila melanogaster., Frontiers in Molecular Neuroscience, 2(11):1-6.

Kazama H \& Wilson RI (2008), Homeostatic matching and nonlinear amplification at identified central synapses., Neuron, 58(3):401-413. 


\section{Bibliography}

Keene AC, Krashes MJ, Leung B, Bernard JA \& Waddell S (2006), Drosophila Dorsal Paired Medial Neurons Provide a General Mechanism for Memory Consolidation., Current Biology, 16(15):1524-1530.

Keene AC, Stratmann M, Keller A, Perrat PN, Vosshall LB \& Waddell S (2004), Diverse odor-conditioned memories require uniquely timed dorsal paired medial neuron output., Neuron, 44(3):521-533.

Keene AC \& Waddell S (2007), Drosophila olfactory memory: single genes to complex neural circuits., Nature Reviews Neuroscience, 8(5):341-354.

Kim YC, Lee HG \& Han KA (2007), D1 dopamine receptor dDA1 is required in the mushroom body neurons for aversive and appetitive learning in Drosophila., The Journal of neuroscience, 27(29):7640-7647.

Kim YC, Lee HG, Seong CS \& Han KA (2003), Expression of a D1 dopamine receptor dDA1/DmDOP1 in the central nervous system of Drosophila melanogaster<., Gene expression patterns, 3(2):237-245.

Kim YT \& Wu CF (1990), Allelic interactions at the shibire locus of Drosophila: effects on behavior., Journal of Neurogenetics, 7(1):1-14.

Kimmel HD (1977), Notes from" Pavlov's Wednesdays": sensory preconditioning., The American Journal of Psychology, 90(2):319-321.

Kitamoto T (2001), Conditional modification of behavior in Drosophila by targeted expression of a temperature-sensitive shibire allele in defined neurons., Journal of neurobiology, 47(2):81-92.

Klein SB (2002), Learning, McGraw-Hill, New York, ISBN 9780071131438.

Knöpfel T, Díez-García J \& Akemann W (2006), Optical probing of neuronal circuit dynamics: genetically encoded versus classical fluorescent sensors., Trends in Neurosciences, 29(3):160-166.

Koldenkova VP \& Nagai T (2013), Genetically encoded $\mathrm{Ca}^{2+}$ indicators: Properties and evaluation., Biochimica et Biophysica Acta - Molecular Cell Research, http://dx.doi.org/10.1016/j.bbamcr.2013.01.011:pp. 1-11. 


\section{Bibliography}

Kosaka T \& Ikeda K (1983), Reversible blockage of membrane retrieval and endocytosis in the garland cell of the temperature-sensitive mutant of Drosophila melanogaster, shibire ${ }^{t s 1}$., The Journal of cell biology, 97(2):499-507.

Krashes MJ, Keene AC, Leung B, Armstrong JD \& Waddell S (2007), Sequential Use of Mushroom Body Neuron Subsets during Drosophila Odor Memory Processing., Neuron, 53(1):103-115.

Kremer MC, Christiansen F, Leiss F, Paehler M, Knapek S, Andlauer TFM, Förstner F, Kloppenburg P, Sigrist SJ \& Tavosanis G (2010), Structural Long-Term Changes at Mushroom Body Input Synapses., Current Biology, 20(21):1938-1944.

Kwon JY, Dahanukar A, Weiss LA \& Carlson JR (2007), The molecular basis of $\mathrm{CO}_{2}$ reception in Drosophila., Proceedings of the National Academy of Sciences, 104(9):3574-3578.

Lai SL \& Lee T (2006), Genetic mosaic with dual binary transcriptional systems in Drosophila., Nature Neuroscience, 9(5):703-709.

Laissue PP, Reiter C, Hiesinger PR, Halter S, Fischbach KF \& Stocker RF (1999), Three-dimensional reconstruction of the antennal lobe in Drosophila melanogaster., The Journal of Comparative Neurology, 405(4):543-552.

Larsson MC, Domingos Al, Jones WD, Chiappe ME, Amrein H \& Vosshall LB (2004), Or83b encodes a broadly expressed odorant receptor essential for Drosophila olfaction., Neuron, 43(5):703-714.

Lashley KS \& Wade M (1946), The Pavlovian theory of generalization., Psychological review, 53:72-87.

Le Bourg É (2004), Effects of aging on learned suppression of photopositive tendencies in Drosophila melanogaster., Neurobiology of aging, 25(9):1241-1252.

Lee PT, Lin HW, Chang YH, Fu TF, Dubnau J, Hirsh J, Lee T \& Chiang AS (2011), Serotonin,Äìmushroom body circuit modulating the formation of anesthesiaresistant memory in Drosophila., Proceedings of the National Academy of Sciences, 108(33):13794-13799. 


\section{Bibliography}

Leinwand SG \& Chalasani SH (2011), Olfactory networks: from sensation to perception., Current Opinion in Genetics \& Development, 21(6):806-811.

Leiss F, Groh C, Butcher NJ, Meinertzhagen IA \& Tavosanis G (2009), Synaptic organization in the adult Drosophila mushroom body calyx., The Journal of Comparative Neurology, 517(6):808-824.

Li W, Howard JD, Parrish TB \& Gottfried JA (2008), Aversive Learning Enhances Perceptual and Cortical Discrimination of Indiscriminable Odor Cues., Science, 319(5871):1842-1845.

Liang L \& Luo L (2010), The olfactory circuit of the fruit fly Drosophila melanogaster., Science China Life Sciences, 53(4):472-484.

Lima SQ \& Miesenböck G (2005), Remote Control of Behavior through Genetically Targeted Photostimulation of Neurons., Cell, 121(1):141-152.

Lin HH, Lai JSY, Chin AL, Chen YC \& Chiang AS (2007), A Map of Olfactory Representation in the Drosophila Mushroom Body., Cell, 128(6):1205-1217.

Lindenburg L \& Merkx M (2012), Colorful Calcium Sensors., ChemBioChem, 13(3):349-351.

Linster C \& Smith BH (1999), Generalization between binary odor mixtures and their components in the rat., Physiology \& behavior, 66(4):701-707.

Liu C, Plaçais PY, Yamagata N, Pfeiffer BD, Aso Y, Friedrich AB, Siwanowicz I, Rubin GM, Preat T \& Tanimoto H (2012), A subset of dopamine neurons signals reward for odour memory in Drosophila., Nature, 488:512-516.

Liu X \& Davis RL (2009), The GABAergic anterior paired lateral neuron suppresses and is suppressed by olfactory learning., Nature Neuroscience, 12(1):53-59.

Mao Z, Roman G, Zong L \& Davis RL (2004), Pharmacogenetic rescue in time and space of the rutabaga memory impairment by using Gene-Switch., Proceedings of the National Academy of Sciences, 101(1):198-203.

Margulies C, Tully T \& Dubnau J (2005), Deconstructing Memory in Drosophila., Current Biology, 15(17):R700-R713. 
Marin EC, Jefferis GS, Komiyama T, Zhu H \& Luo L (2002), Representation of the Glomerular Olfactory Map in the Drosophila Brain, Cell, 109(2):243-255.

Masek P \& Heisenberg M (2008), Distinct memories of odor intensity and quality in Drosophila., Proceedings of the National Academy of Sciences of the United States of America, 105(41):15985-15990.

Masse NY, Turner GC \& Jefferis GSXE (2009), Olfactory Information Processing in Drosophila., Current Biology, 19(16):R700-R713.

Matsumoto Y, Hirashima D \& Mizunami M (2013), Analysis and modeling of neural processes underlying sensory preconditioning., Neurobiology of Learning and Memory, 101(0):103 - 113.

McGuire SE, Deshazer M \& Davis RL (2005), Thirty years of olfactory learning and memory research in Drosophila melanogaster., Progress in Neurobiology, 76(5):328-347.

McGuire SE, Le PT, Osborn AJ, Matsumoto K \& Davis RL (2003), Spatiotemporal rescue of memory dysfunction in Drosophila., Science Signaling, 302(5651):1765-1768.

Miller RR, Barnet RC \& Grahame NJ (1995), Assessment of the Rescorla-Wagner model., Psychological Bulletin, 117(3):363-386.

Mishra D, Louis M \& Gerber B (2010), Adaptive Adjustment of the GeneralizationDiscrimination Balance in Larval Drosophila., Journal of Neurogenetics, 24(3):168-175.

Miyawaki A, Llopis J, Heim R, McCaffery JM, Adams JA, Ikurak M \& Tsien RY (1997), Fluorescent indicators for $\mathrm{Ca}^{2+}$ based on green fluorescent proteins and calmodulin., Nature, 388:882-887.

Müller D, Gerber B, Hellstern F, Hammer M \& Menzel R (2000), Sensory preconditioning in honeybees., The Journal of experimental biology, 203(Pt 8):1351-1364.

Murthy M, Fiete I \& Laurent G (2008), Testing Odor Response Stereotypy in the Drosophila Mushroom Body., Neuron, 59(6):1009-1023. 
Nagai T, Sawano A, Park ES \& Miyawaki A (2001), Circularly permuted green fluorescent proteins engineered to sense $\mathrm{Ca}^{2+}$., Proceedings of the National Academy of Sciences, 98(6):3197-3202.

Nagel G, Szellas T, Huhn W, Kateriya S, Adeishvili N, Berthold P, Ollig D, Hegemann P \& Bamberg E (2003), Channelrhodopsin-2, a directly light-gated cationselective membrane channel., Proceedings of the National Academy of Sciences, 100(24):13940-13945.

Nakagawa T \& Vosshall LB (2009), Controversy and consensus: noncanonical signaling mechanisms in the insect olfactory system., Current opinion in neurobiology, 19(3):284-292.

Nakai J, Ohkura M \& Imoto K (2001), A high signal-to-noise $\mathrm{Ca}^{2+}$ probe composed of a single green fluorescent protein., Nature Biotechnology, 19(2):137-141.

Neuhaus EM, Gisselmann G, Zhang W, Dooley R, Störtkuhl K \& Hatt H (2005), Odorant receptor heterodimerization in the olfactory system of Drosophila melanogaster, Nature neuroscience, 8(1):15-17.

Neuser K, Triphan T, Mronz M, Poeck B \& Strauss R (2008), Analysis of a spatial orientation memory in Drosophila., Nature, 453(7199):1244-1247.

Ng M, Roorda RD, Lima SQ, Zemelman BV, Morcillo P \& Miesenböck G (2002), Transmission of olfactory information between three populations of neurons in the antennal lobe of the fly., Neuron, 36(3):463-474.

Niewalda T, Völler T, Eschbach C, Ehmer J, Chou WC, Timme M, Fiala A \& Gerber B (2011), A Combined Perceptual, Physico-Chemical, and Imaging Approach to 'Odour-Distances' Suggests a Categorizing Function of the Drosophila Antennal Lobe., PLoS ONE, 6(9):e24300.

Nitabach MN, Blau J \& Holmes TC (2002), Electrical silencing of Drosophila pacemaker neurons stops the free-running circadian clock., Cell, 109(4):485-495.

Ohkura M, Matsuzaki M, Kasai H, Imoto K \& Nakai J (2005), Genetically Encoded Bright $\mathrm{Ca}^{2+}$ Probe Applicable for Dynamic $\mathrm{Ca}^{2+}$ Imaging of Dendritic Spines., Analytical Chemistry, 77(18):5861-5869. 


\section{Bibliography}

Okada R, Awasaki T \& Ito K (2009), Gamma-aminobutyric acid (GABA)-mediated neural connections in the Drosophila antennal lobe., The Journal of Comparative Neurology, 514(1):74-91.

Olsen SR \& Wilson RI (2008), Cracking neural circuits in a tiny brain: new approaches for understanding the neural circuitry of Drosophila., Trends in neurosciences, 31(10):512-520.

Pascual A (2001), Localization of Long-Term Memory Within the Drosophila Mushroom Body., Science, 294(5544):1115-1117.

Pavlov IP (1927), Conditioned Reflexes, Courier Dover Publications, ISBN 9780486430935.

Pearce JM (1994), Similarity and discrimination: a selective review and a connectionist model., Psychological review, 101(4):587-607.

Peele P, Ditzen M, Menzel R \& Galizia CG (2006), Appetitive odor learning does not change olfactory coding in a subpopulation of honeybee antennal lobe neurons., Journal of comparative physiology. A, Sensory, neural, and behavioral physiology, 192(10):1083-1103.

Perez-Orive J (2002), Oscillations and Sparsening of Odor Representations in the Mushroom Body., Science, 297(5580):359-365.

Pfeiffer BD, Ngo TTB, Hibbard KL, Murphy C, Jenett A, Truman JW \& Rubin GM (2010), Refinement of Tools for Targeted Gene Expression in Drosophila., Genetics, 186(2):735-755.

Pitman JL, DasGupta S, Krashes MJ, Leung B, Perrat PN \& Waddell S (2009), There are many ways to train a fly., Fly, 3(1):3-9.

Pitman JL, Huetteroth W, Burke CJ, Krashes MJ, Lai SL, Lee T \& Waddell S (2011), A Pair of Inhibitory Neurons Are Required to Sustain Labile Memory in the Drosophila Mushroom Body., Current biology : CB, 21(10):855-861.

Potter CJ, Tasic B, Russler EV, Liang L \& Luo L (2010), The Q System: A Repressible Binary System for Transgene Expression, Lineage Tracing, and Mosaic Analysis., Cell, 141(3):536-548. 


\section{Bibliography}

Pulver SR, Pashkovski SL, Hornstein NJ, Garrity PA \& Griffith LC (2009), Temporal Dynamics of Neuronal Activation by Channelrhodopsin-2 and TRPA1 Determine Behavioral Output in Drosophila Larvae., Journal of Neurophysiology, 101(6):3075-3088.

Qin H, Cressy M, Li W, Coravos JS, Izzi SA \& Dubnau J (2012), Gamma Neurons Mediate Dopaminergic Input during Aversive Olfactory Memory Formation in Drosophila., Current biology, 22(7):608-614.

Quinn WG \& Dudai Y (1976), Memory phases in Drosophila., Nature, 262:576-577.

Quinn WG, Harris WA \& Benzer S (1974), Conditioned behavior in Drosophila melanogaster., Proceedings of the National Academy of Sciences of the United States of America, 71(3):708-712.

Quinn WG, Sziber PP \& Booker R (1979), The Drosophila memory mutant amnesiac., Nature, 277:212-214.

R Core Team (2012), R: A Language and Environment for Statistical Computing., $\mathrm{R}$ Foundation for Statistical Computing, Vienna, Austria.

Rescorla RA (1969a), Conditioned inhibition of fear resulting from negative CS-US contingencies., Journal of comparative and physiological psychology, 67(4):504509.

Rescorla RA (1969b), Pavlovian conditioned inhibition., Psychological Bulletin, $72(2): 77$.

Rescorla RA (1973), Evidence for ,Äúunique stimulus,Äù account of configural conditioning., Journal of Comparative and Physiological Psychology, 85(2):331-338.

Rescorla RA (1980), Simultaneous and successive associations in sensory preconditioning., Journal of experimental psychology. Animal behavior processes, 6(3):207-216.

Rescorla RA \& Wagner AR (1972), A Theory of Pavlovian Conditioning: Variations in the Effectiveness of Reinforcement and Nonreinforcement., in: $\mathrm{AH}$ Black 


\section{Bibliography}

\& WF Prokasy (Editors), Classical Conditioning II: current research and theory, pp. 64-99, New York: Appleton-Century-Crofts.

Riemensperger T, Pech U, Dipt S \& Fiala A (2012), Optical calcium imaging in the nervous system of Drosophila melanogaster., Biochimica et Biophysica Acta General Subjects, 1820(8):1169 - 1178.

Riemensperger T, Völler T, Stock P, Buchner E \& Fiala A (2005), Punishment Prediction by Dopaminergic Neurons in Drosophila., Current Biology, 15(21):19531960.

Rodrigues V (1988), Spatial coding of olfactory information in the antennal lobe of Drosophila melanogaster., Brain research, 453(1):299-307.

Ronderos DS \& Smith DP (2009), Diverse signaling mechanisms mediate volatile odorant detection in Drosophila., Fly, 3(4):290-297.

Rubin BD \& Katz LC (1999), Optical Imaging of Odorant Representations in the Mammalian Olfactory Bulb., Neuron, 23(3):499 - 511.

Rubin GM \& Spradling AC (1982), Genetic transformation of Drosophila with transposable element vectors., Science, 218(4570):348-353.

Sachse S \& Galizia CG (2002), Role of inhibition for temporal and spatial odor representation in olfactory output neurons: a calcium imaging study., Journal of Neurophysiology, 87(2):1106-1117.

Sachse S, Rueckert E, Keller A, Okada R, Tanaka NK, Ito K \& Vosshall LB (2007), Activity-Dependent Plasticity in an Olfactory Circuit., Neuron, 56(5):838850.

Saiki RK, Gelfand DH, Stoffel S, Scharf SJ, Higuchi R, Horn GT, Mullis KB \& Erlich HA (1988), Primer-directed enzymatic amplification of DNA with a thermostable DNA polymerase., Science, 239(4839):487-491.

Saito K, Chang Y, Horikawa K, Hatsugai N, Higuchi Y, Hashida M, Yoshida Y, Matsuda T, Arai Y \& Nagai T (2012), Luminescent proteins for high-speed singlecell and whole-body imaging., Nature communications, 3:1262. 


\section{Bibliography}

Saito K, Hatsugai N, Horikawa K, Kobayashi K, Matsu-ura T, Mikoshiba K \& Nagai T (2010), Auto-Luminescent Genetically-Encoded Ratiometric Indicator for Real-Time $\mathrm{Ca}^{2+}$ Imaging at the Single Cell Level., PLoS ONE, 5(4):e9935.

Salzberg BM, Davila HV \& Cohen LB (1973), Optical recording of impulses in individual neurones of an invertebrate central nervous system., Nature, 246(5434):508-509.

Sato K, Pellegrino M, Nakagawa T, Nakagawa T, Vosshall LB \& Touhara K (2008), Insect olfactory receptors are heteromeric ligand-gated ion channels., $\mathrm{Na}$ ture, 452(7190):1002-1006.

Saumweber T, Husse J \& Gerber B (2011), Innate Attractiveness and Associative Learnability of Odors Can Be Dissociated in Larval Drosophila., Chemical Senses, 36(3):223-235.

Savastano HI, Cole RP, Barnet RC \& Miller RR (1999), Reconsidering conditioned inhibition., Learning and Motivation, 30(1):101-127.

Scheunemann L, Jost E, Richlitzki A, Day JP, Sebastian S, Thum AS, Efetova M, Davies SA \& Schwärzel M (2012), Consolidated and labile odor memory are separately encoded within the Drosophila brain., The Journal of Neuroscience, 32(48):17163-17171.

Schmuker M \& Schneider G (2007), Processing and classification of chemical data inspired by insect olfaction., Proceedings of the National Academy of Sciences of the United States of America, 104(51):20285-20289.

Schneider CA, Rasband WS \& Eliceiri KW (2012), NIH Image to ImageJ: 25 years of image analysis., Nature Methods, 9(7):671-675.

Schroll C, Riemensperger T, Bucher D, Ehmer J, Völler T, Erbguth K, Gerber B, Hendel T, Nagel G, Buchner E \& Fiala A (2006), Light-Induced Activation of Distinct Modulatory Neurons Triggers Appetitive or Aversive Learning in Drosophila Larvae., Current Biology, 16(17):1741-1747. 
Schulz RA, Chromey C, Lu MF, Zhao B \& Olson EN (1996), Expression of the D-MEF2 transcription in the Drosophila brain suggests a role in neuronal cell differentiation., Oncogene, 12(8):1827-1831.

Schwaerzel M, Heisenberg M \& Zars T (2002), Extinction antagonizes olfactory memory at the subcellular level., Neuron, 35(5):951-960.

Schwaerzel M, Monastirioti M, Scholz H, Friggi-Grelin F, Birman S \& Heisenberg $\mathbf{M}$ (2003), Dopamine and octopamine differentiate between aversive and appetitive olfactory memories in Drosophila., Journal of Neuroscience, 23(33):10495-10502.

Séjourné J, Plaçais PY, Aso Y, Siwanowicz I, Trannoy S, Thoma V, Tedjakumala SR, Rubin GM, Tchénio P, Ito K, Isabel G, Tanimoto H \& Preat T (2011), Mushroom body efferent neurons responsible for aversive olfactory memory retrieval in Drosophila., Nature Publishing Group, 14(7):903-910.

Seki Y, Rybak J, Wicher D, Sachse S \& Hansson BS (2010), Physiological and Morphological Characterization of Local Interneurons in the Drosophila Antennal Lobe., Journal of Neurophysiology, 104(2):1007-1019.

Selcho M, Pauls D, Han KA, Stocker RF \& Thum AS (2009), The Role of Dopamine in Drosophila Larval Classical Olfactory Conditioning., PLOS ONE, 4(6):e5897.

Shanbhag SR, Müller B \& Steinbrecht RA (1999), Atlas of olfactory organs of Drosophila melanogaster: 1. Types, external organization, innervation and distribution of olfactory sensilla., International Journal of Insect Morphology and Embryology, 28(4):377-397.

Shang Y, Claridge-Chang A, Sjulson L, Pypaert M \& Miesenböck G (2007), Excitatory Local Circuits and Their Implications for Olfactory Processing in the Fly Antennal Lobe., Cell, 128(3):601-612.

Shepard RN (1987), Toward a universal law of generalization for psychological science., Science, 237(4820):1317-1323. 


\section{Bibliography}

Shiga Y, Tanaka-Matakatsu M \& Hayashi S (1996), A nuclear GFP/ $\beta$-galactosidase fusion protein as a marker for morphogenesis in living Drosophila., Development, Growth \& Differentiation, 38(1):99-106.

Siegel RW \& Hall JC (1979), Conditioned responses in courtship behavior of normal and mutant Drosophila., Proceedings of the National Academy of Sciences, 76(7):3430-3434.

Silbering AF \& Galizia CG (2007), Processing of Odor Mixtures in the Drosophila Antennal Lobe Reveals both Global Inhibition and Glomerulus-Specific Interactions., Journal of Neuroscience, 27(44):11966-11977.

Skoulakis E, Kalderon D \& Davis RL (1993), Preferential expression in mushroom bodies of the catalytic subunit of protein kinase $A$ and its role in learning and memory., Neuron, 11(2):197-208.

Spall T (2005), Optische Visualisierung neuronaler Aktivität: Etablierung des in - vivo Calcium Imaging mit dem genetisch codierten Sensor Yellow Cameleon 2.1und Untersuchung der olfaktorischen Codierung im Gehirn von Drosophila melanogaster., Ph.D. thesis, Bayerischen Julius-MaximiliansUniversität Würzburg.

Stallcup WB (1979), Sodium and calcium fluxes in a clonal nerve cell line., The Journal of physiology, 286:525-540.

Stocker RF (2001), Drosophila as a focus in olfactory research: mapping of olfactory sensilla by fine structure, odor specificity, odorant receptor expression, and central connectivity., Microscopy research and technique, 55(5):284-296.

Stocker RF, Heimbeck G, Gendre N \& de Belle JS (1997), Neuroblast ablation in Drosophila P [GAL4] lines reveals origins of olfactory interneurons., Journal of neurobiology, 32(5):443-456.

Stocker RF, Lienhard MC, Borst A \& Fischbach KF (1990), Neuronal architecture of the antennal lobe in Drosophila melanogaster., Cell and tissue research, 262(1):9. 


\section{Bibliography}

Stopfer M, Bhagavan S, Smith BH \& Laurent G (1997), Impaired odour discrimination on desynchronization of odour-encoding neural assemblies., Nature, 390(6655):70-74.

Strausfeld NJ, Sinakevitch I \& Vilinsky I (2003), The mushroom bodies of Drosophila melanogaster: An immunocytological and golgi study of Kenyon cell organization in the calyces and lobes., Microscopy Research and Technique, 62(2):151-169.

Su CY, Menuz K \& Carlson JR (2009), Olfactory Perception: Receptors, Cells, and Circuits., Cell, 139(1):45-59.

Sudhakaran IP, Holohan EE, Osman S, Rodrigues V, VijayRaghavan K \& Ramaswami M (2012), Plasticity of Recurrent Inhibition in the Drosophila Antennal Lobe., Journal of Neuroscience, 32(21):7225-7231.

Suh GS, Wong AM, Hergarden AC, Wang JW, Simon AF, Benzer S, Axel R \& Anderson DJ (2004), A single population of olfactory sensory neurons mediates an innate avoidance behaviour in Drosophila., Nature, 431(7010):854-859.

Svedlow M, McGillem CD \& Anuta PE (1976), Experimental examination of similarity measures and preprocessing methods used for image registration., LARS Symposia, Paper 150.

Sweeney ST, Broadie K, Keane J, Niemann H \& O’Kane CJ (1995), Targeted expression of tetanus toxin light chain in Drosophila specifically eliminates synaptic transmission and causes behavioral defects., Neuron, 14(2):341-351.

Szüts D \& Bienz M (2000), LexA chimeras reveal the function of Drosophila Fos as a context-dependent transcriptional activator., Proceedings of the National Academy of Sciences, 97(10):5351-5356.

Szyszka P, Ditzen M, Galkin A, Galizia CG \& Menzel R (2005), Sparsening and temporal sharpening of olfactory representations in the honeybee mushroom bodies., Journal of neurophysiology, 94(5):3303-3313. 


\section{Bibliography}

Szyszka P, Galkin A \& Menzel R (????), Associative and non-associative plasticity in Kenyon cells of the honeybee mushroom body., Frontiers in systems neuroscience, 2(3):1-10.

Tabone CJ \& de Belle JS (2011), Second-order conditioning in Drosophila., Learning \& Memory, 18(4):250-253.

Tabor R, Yaksi E, Weislogel JM \& Friedrich RW (2004), Processing of odor mixtures in the zebrafish olfactory bulb., The Journal of neuroscience, 24(29):66116620.

Tanaka NK, Awasaki T, Shimada T \& Ito K (2004), Integration of Chemosensory Pathways in the Drosophila Second-Order Olfactory Centers., Current biology, 14(6):449-457.

Tanaka NK, Endo K \& Ito K (2012), Organization of antennal lobe-associated neurons in adult Drosophila melanogaster brain., The Journal of Comparative Neurology, 520(18):4067-4130.

Tanaka NK, Ito K \& Stopfer M (2009), Odor-Evoked Neural Oscillations in Drosophila Are Mediated by Widely Branching Interneurons., Journal of Neuroscience, 29(26):8595-8603.

Tanaka NK, Tanimoto H \& Ito K (2008), Neuronal assemblies of the Drosophila mushroom body., The Journal of Comparative Neurology, 508(5):711-755.

Tang S, Wong HC, Wang ZM, Huang Y, Zou J, Zhuo Y, Pennati A, Gadda G, Delbono $\mathbf{O}$ \& Yang JJ (2011), Design and application of a class of sensors to monitor $\mathrm{Ca}^{2+}$ dynamics in high $\mathrm{Ca}^{2+}$ concentration cellular compartments., Proceedings of the National Academy of Sciences, 108(39):16265-16270.

Technau G \& Heisenberg M (1982), Neural reorganization during metamorphosis of the corpora pedunculata in Drosophila melanogaster., Nature, 295:405-407.

Tempel BL, Bonini N, Dawson DR \& Quinn WG (1983), Reward learning in normal and mutant Drosophila., Proceedings of the National Academy of Sciences, 80(5):1482-1486. 


\section{Bibliography}

Thévenaz P, Ruttimann UE \& Unser M (1998), A pyramid approach to subpixel registration based on intensity., IEEE transactions on image processing : a publication of the IEEE Signal Processing Society, 7(1):27-41.

Tian L, Hires SA, Mao T, Huber D, Chiappe ME, Chalasani SH, Petreanu L, Akerboom J, McKinney SA, Schreiter ER, Bargmann Cl, Jayaraman V, Svoboda K \& Looger LL (2009), Imaging neural activity in worms, flies and mice with improved GCaMP calcium indicators., Nature Methods, 6(12):875-881.

Tomchik SM \& Davis RL (2009), Dynamics of Learning-Related cAMP Signaling and Stimulus Integration in the Drosophila Olfactory Pathway, Neuron, 64(4):510521.

Tootoonian S \& Laurent G (2010), Electric Times in Olfaction., Neuron, 67(6):903905.

Trannoy S, Redt-Clouet C, Dura JM \& Preat T (2011), Parallel Processing of Appetitive Short- and Long-Term Memories In Drosophila., Current biology : $C B$, 21(19):1647-1653.

Tully T, Preat T, Boynton S \& Del Vecchio M (1994), Genetic dissection of consolidated memory in Drosophila., Cell, 79(1):35-47.

Tully T \& Quinn WG (1985), Classical conditioning and retention in normal and mutant Drosophila melanogaster., Journal of comparative physiology. A, Sensory, neural, and behavioral physiology, 157(2):263-277.

Turner GC, Bazhenov M \& Laurent G (2008), Olfactory Representations by Drosophila Mushroom Body Neurons, Journal of Neurophysiology, 99(2):734746 .

van der Bliek AM \& Meyerowitz EM (1991), Dynamin-like protein encoded by the Drosophila shibire gene associated with vesicular traffic., Nature, 351(6325):411414.

Venken KJT, Simpson JH \& Bellen HJ (2011), Genetic Manipulation of Genes and Cells in the Nervous System of the Fruit Fly., Neuron, 72(2):202-230. 


\section{Bibliography}

Vosshall LB, Amrein H, Morozov PS, Rzhetsky A \& Axel R (1999), A Spatial Map of Olfactory Receptor Expression in the emphDrosophila Antenna., Cell, 96(5):725 $-736$.

Vosshall LB \& Stocker RF (2007), Molecular Architecture of Smell and Taste in Drosophila., Annual Review of Neuroscience, 30(1):505-533.

Vosshall LB, Wong AM \& Axel R (2000), An olfactory sensory map in the fly brain., Cell, 102(2):147-159.

Waddell S (2013), Reinforcement signalling in Drosophila; dopamine does it all after all., Current Opinion in Neurobiology, http://dx.doi.org/10.1016/j.conb.2013. 01.005:pp. 1-6.

Waddell S, Armstrong JD, Kitamoto T, Kaiser K \& Quinn WG (2000), The amnesiac gene product is expressed in two neurons in the Drosophila brain that are critical for memory., Cell, 103(5):805-813.

Waddell S \& Quinn WG (2001), What can we teach Drosophila? What can they teach us?, Trends in genetics, 17(12):719-726.

Wadhams GH \& Armitage JP (2004), Making sense of it all: bacterial chemotaxis., Nature Reviews Molecular Cell Biology, 5(12):1024-1037.

Wagh DA, Rasse TM, Asan E, Hofbauer A, Schwenkert I, Dürrbeck H, Buchner S, Dabauvalle MC, Schmidt M \& Qin G (2006), Bruchpilot, a Protein with Homology to ELKS/CAST, Is Required for Structural Integrity and Function of Synaptic Active Zones in Drosophila., Neuron, 49(6):833-844.

Wang JW, Wong AM, Flores J, Vosshall LB \& Axel R (2003a), Two-photon calcium imaging reveals an odor-evoked map of activity in the fly brain., Cell, 112(2):271282.

Wang Y, Chiang AS, Xia S, Kitamoto T, Tully T \& Zhong Y (2003b), Blockade of Neurotransmission in Drosophila Mushroom Bodies Impairs Odor Attraction, but Not Repulsion., Current Biology, 13(21):1900-1904. 
Wang Y, Guo HF, Pologruto TA, Hannan F, Hakker I, Svoboda K \& Zhong Y (2004), Stereotyped odor-evoked activity in the mushroom body of Drosophila revealed by green fluorescent protein-based $\mathrm{Ca}^{2+}$ imaging., The Journal of neuroscience, 24(29):6507-6514.

Wang Y, Mamiya A, Chiang AS \& Zhong Y (2008), Imaging of an Early Memory Trace in the Drosophila Mushroom Body., Journal of Neuroscience, 28(17):43684376.

Wessnitzer J, Young JM, Armstrong JD \& Webb B (2011), A model of nonelemental olfactory learning in Drosophila., Journal of Computational Neuroscience, 32(2):197-212.

White B \& Peabody NC (2009), Neurotrapping: cellular screens to identify the neural substrates of behavior in Drosophila., Frontiers in Molecular Neuroscience, 2(20):1-9.

Wicher D, Schäfer R, Bauernfeind R, Stensmyr MC, Heller R, Heinemann SH \& Hansson BS (2008), Drosophila odorant receptors are both ligand-gated and cyclic-nucleotide-activated cation channels., Nature, 452(7190):1007-1011.

Wilson DA \& Stevenson RJ (2006), Learning to smell: olfactory perception from neurobiology to behavior, The John Hopkins University Press, Baltimore, ISBN 0801883687.

Wilson RI (2004), Transformation of Olfactory Representations in the Drosophila Antennal Lobe., Science, 303(5656):366-370.

Wilson RI (2005), Role of GABAergic Inhibition in Shaping Odor-Evoked Spatiotemporal Patterns in the Drosophila Antennal Lobe., Journal of Neuroscience, 25(40):9069-9079.

Wright GA (2004), Different Thresholds for Detection and Discrimination of Odors in the Honey bee (Apis mellifera)., Chemical Senses, 29(2):127-135.

Wright GA, Kottcamp SM \& Thomson MGA (2008), Generalization Mediates Sensitivity to Complex Odor Features in the Honeybee., PLOS ONE, 3(2):e1704. 


\section{Bibliography}

Wright GA, Skinner BD \& Smith BH (2002), Ability of honeybee, Apis mellifera, to detect and discriminate odors of varieties of canola (Brassica rapa and Brassica napus) and snapdragon flowers (Antirrhinum majus)., Journal of chemical ecology, 28(4):721-740.

Wu CL, Shih MFM, Lai JSY, Yang HT, Turner GC, Chen L \& Chiang AS (2011), Heterotypic Gap Junctions between Two Neurons in the Drosophila Brain Are Critical for Memory., Current biology, 21(10):848-854.

Xia S, Miyashita T, Fu TF, Lin WY, Wu CL, Pyzocha L, Lin IR, Saitoe M, Tully T \& Chiang AS (2005), NMDA Receptors Mediate Olfactory Learning and Memory in Drosophila., Current biology, 15(7):603-615.

Yaksi E \& Wilson RI (2010), Electrical Coupling between Olfactory Glomeruli., Neuron, 67(6):1034-1047.

Yao Yang M, Armstrong JD, Vilinsky I, Strausfeld NJ \& Kaiser K (1995), Subdivision of the Drosophila mushroom bodies by enhancer-trap expression patterns., Neuron, 15(1):45-54.

Yasuyama K, Meinertzhagen IA \& Sch rmann FW (2003), Synaptic connections of cholinergic antennal lobe relay neurons innervating the lateral horn neuropile in the brain of Drosophila melanogaster., The Journal of Comparative Neurology, 466(3):299-315.

Yasuyama K, Meinertzhagen IA \& Schürmann FW (2002), Synaptic organization of the mushroom body calyx in Drosophila melanogaster, The Journal of Comparative Neurology, 445(3):211-226.

Yin J, Wallach J, Del Vecchio M, Wilder E, Zhou H, Quinn W \& Tully T (1994), Induction of a dominant negative CREB transgene specifically blocks long-term memory in Drosophila., Cell, 79(1):49-58.

Young JM, Wessnitzer J, Armstrong JD \& Webb B (2011), Elemental and nonelemental olfactory learning in Drosophila., Neurobiology of learning and memory, 96(2):339-352. 
Yu D, Akalal DBG \& Davis RL (2006), Drosophila $\alpha / \beta$ Mushroom Body Neurons Form a Branch-Specific, Long-Term Cellular Memory Trace after Spaced Olfactory Conditioning., Neuron, 52(5):845-855.

Yu D, Keene AC, Srivatsan A, Waddell S \& Davis RL (2005), Drosophila DPM Neurons Form a Delayed and Branch-Specific Memory Trace after Olfactory Classical Conditioning., Cell, 123(5):945-957.

Yu D, Ponomarev A \& Davis RL (2004), Altered representation of the spatial code for odors after olfactory classical conditioning; memory trace formation by synaptic recruitment., Neuron, 42(3):437-449.

Zars T (2010), Short-term memories in Drosophila are governed by general and specific genetic systems., Learning \& Memory, 17(5):246-251.

Zars T, Fischer M, Schulz R \& Heisenberg M (2000), Localization of a short-term memory in Drosophila., Science, 288(5466):672-675.

Zeidler MP, Tan C, Bellaiche Y, Cherry S, Häder S, Gayko U \& Perrimon N (2004), Temperature-sensitive control of protein activity by conditionally splicing inteins., Nature Biotechnology, 22(7):871-876.

Zhang F, Wang LP, Brauner M, Liewald JF, Kay K, Watzke N, Wood PG, Bamberg E, Nagel G, Gottschalk A \& Deisseroth K (2007), Multimodal fast optical interrogation of neural circuitry., Nature, 446(7136):633-639.

Zhao Y, Araki S, Wu J, Teramoto T, Chang YF, Nakano M, Abdelfattah AS, Fujiwara M, Ishihara T, Nagai T \& Campbell RE (2011), An Expanded Palette of Genetically Encoded Ca ${ }^{2+}$ Indicators., Science, 333(6051):1888-1891. 


\section{Acknowledgements}

First of all, I want to thank Prof. Dr. André Fiala for his great supervision and the opportunity to conduct this thesis in his laboratory. I am very grateful that he always found the time to help, discuss and give advice. Even during my non-scientific problems with the university administration concerning my non-existing Masters degree, he supported me and used the lab budget to overcome the financial difference that arose.

I also want to thank my second reviewer, Prof. Andreas Wodarz, and the third member of my thesis committee, Prof. Andre Fischer, for their time and helpful comments during the thesis committee meetings.

I am thankful to all members of the Department of Molecular Neurobiology of Behavior, former and present, for their friendship and assistance during the last three and a half years: Abud, Atefeh, David, Jutta, Mandy, Moritz, Priyanka, Sandra, Shubham, Simon, Tom and Uli. Thanks also to the numerous Bachelor and Master students who spend time in our lab and produced a constant fluctuation of lab members with different perspectives and ideas. Special thanks to Shubham for all his help with the functional imaging and especially during the analysis of the mushroom body imaging data. Without him and his mathematical and computational skills, the data evaluation would have taken much more time or even be impossible. To "my" Bachelor student Moritz who conducted some of the behavioral experiments together with me during an internship and his bachelor thesis. To Tom for all his advice in the last years of the thesis and his help during the anatomical characterization of the driver lines. To Uli for her help during the evaluation and of the GECO flies, her profound knowledge about the mushroom body which she was very willing to share and her advice on this manuscript. To Simon and Atefeh for sharing an office with me and tolerating (and sometimes even participating in) my occasional outbursts of insanity. 


\section{Bibliography}

Thanks to Prof. Dr. Michael Hörner and Sandra Drube for their support and assistance during my four and a half years in the IMPRS Neuroscience. They always had an open door for my administrative problems and helped in so many ways. Additional thanks to the GGNB for a great organization of methods and skill courses and the bridging fund that supported me financially for the last five month of the thesis.

I am also very grateful for the support from all my friends. Leute, I especially want to thank you for being such awesome people and all the time we spend together, getting our minds of science and putting them at rest. Alwaleed, Andrea, Andrew, Derya, Liam, Matthieu, Natalia, Sanaz and Steph thanks for awesome dinners, fabulous evenings and exciting trips all around Europe. Thanks to Ania for relaxing coffee breaks and deep conversations about life and the future. To Sünke and Alonso for being great friends and taking me with them around the world when I needed a break. To Roman, Benni and Cordelia for their friendship and taking the German language into my life again.

Special thanks also to Sona for her love and great support! Dear, I do not know what I would have done without you especially in the last months during which you took care that I eat properly, motivated me and provided distraction when needed.

Last but not least, I want to express my gratitude to my parents, Birgit und Uli. Thanks for the continuous support during my whole studies in Giessen and Göttingen! Ihr hattet immer ein offenes Ohr für meine Probleme und wusstet, wie ihr mich wieder aufmuntern und motivieren konntet, ohne mich dabei jemals unter Druck zu setzen. Vielen Dank für alles! 


\section{A. Abbreviations}

$\begin{array}{ll}{ }^{\circ} \text { C } & \text { degrees celcius } \\ \text { 1-Oct } & \text { 1-octen-3-ol } \\ \text { 3-Oct } & \text { 3-octanol } \\ \text { 5-HT } & \text { 5-hydroxytryptamine (serotonin) } \\ \text { AC } & \text { adenylat cyclase } \\ \text { AL } & \text { antennal lobe } \\ \text { APL } & \text { anterior paired lateral (neuron) } \\ \text { ARM } & \text { anesthesia sensitive memory } \\ \text { ASM } & \text { anesthesia resistant memory } \\ \text { ATP } & \text { adenosine triphosphate } \\ \text { BA } & \text { benzaldehyde } \\ \text { Ca }{ }^{2+} & \text { calcium ion } \\ \text { CAMP } & \text { cyclic adenosine monophosphate } \\ \text { CO } 2 & \text { carbon dioxide } \\ \text { CREB } & \text { cAMP response element-binding protein } \\ \text { CR } & \text { conditioned response } \\ \text { CS } & \text { conditioned stimulus } \\ \text { DA } & \text { dopamine } \\ \text { DAL } & \text { dorsal anterior lateral neuron } \\ \text { DC } & \text { direct current } \\ \text { ddH } & \text { doubled destilled water } \\ \text { DNA } & \text { Deoxyribonucleic acid } \\ & \end{array}$




\section{A. Abbreviations}

\begin{tabular}{|c|c|}
\hline DopR & D1-like dopamine receptors \\
\hline dORK & Drosophila open rectifier $\mathrm{K}+$ channel \\
\hline DPM & dorsal paired medial (neuron) \\
\hline dTRPA1 & drosophila transient receptor potential channel A1 \\
\hline ECFP & enhanced cyan fluorescent protein \\
\hline eLN & excitatory local interneuron \\
\hline et al. & et altri \\
\hline EYFP & enhanced yellow fluorescent protein \\
\hline g & gram \\
\hline GABA & $\gamma$-aminobutyric acid \\
\hline GAD & GAL4 activation domain \\
\hline GAL4 & Galactosidase 4 \\
\hline GAL80 & Galactose/lactose metabolism regulatory protein \\
\hline GECI & genetically encoded calcium indicator \\
\hline GFP & green fluorescent protein \\
\hline hrs & hours \\
\hline hz & hertz \\
\hline iLN & inhibitory local interneuron \\
\hline KC & Kenyon cell \\
\hline LH & lateral horn \\
\hline LN & local interneuron \\
\hline LN1 & type I inhibitory local interneurons \\
\hline LN2 & type II inhibitory local interneurons \\
\hline LTM & long term memory \\
\hline $\mathbf{M}$ & molar \\
\hline MB & mushroom body (corpora peduncula) \\
\hline $\mathrm{MCH}$ & 4-methylcyclohexanol \\
\hline
\end{tabular}




\section{A. Abbreviations}

\begin{tabular}{|c|c|}
\hline NGS & normal goat serum \\
\hline OCT & 3-octanol or 1-octen-3-ol \\
\hline OR & olfactory receptor \\
\hline OSN & olfactory sensory neurons \\
\hline PBS & phosphate buffer saline \\
\hline PCA & principle component analysis \\
\hline PCR & polymerase chain reaction \\
\hline PDE & phosphodiesterase \\
\hline PFA & paraformadehyde \\
\hline PN & projection neuron \\
\hline QF & transcription factor for activation of the $Q$ system for binary expression \\
\hline QS & $\begin{array}{l}\text { inhibitor of the transcription factor } Q F \text { in the } Q \text { system for binary ex- } \\
\text { pression }\end{array}$ \\
\hline QUAS & $\begin{array}{l}\text { binding region of the transcription factor } Q F \text { in the } Q \text { system for binary } \\
\text { expression }\end{array}$ \\
\hline sec & seconds \\
\hline Shits & temperature sensitive shibire allele (dominant dynamin mutation) \\
\hline SNARE & soluble NSF attachment protein receptor \\
\hline STM & short term memory \\
\hline UR & unconditioned response \\
\hline US & unconditioned stimulus \\
\hline V & Volt \\
\hline VP & viral promotor \\
\hline
\end{tabular}




\section{B. Fly crosses}

\section{Flies for behavioral experiments}

Type I inhibitory local interneurons (LN1)

$$
\begin{gathered}
\searrow \quad w^{-}, y ; \frac{N P 1227}{C y O} ;+\bigotimes \quad \sigma^{T} \frac{w^{-}, U A S-s h i^{t s}}{\neg} ;+; U A S-s h i^{t s} \\
\Downarrow \\
\frac{y, w^{-}}{U A S-s h i^{t s}} ; \frac{N P 1227}{+} ; \frac{+}{U A S-s h i^{t s}} \quad / \quad \frac{y, w^{-}}{\neg} ; \frac{N P 1227}{+} ; \frac{+}{U A S-s h i^{t s}} \\
\text { test group expressing shibire }{ }^{\text {ts }} \text { in LN1 neurons }
\end{gathered}
$$

AND

$$
\begin{aligned}
& \frac{y, w^{-}}{U A S-s h i^{t t^{2}}} ; \frac{C y O}{+} ; \frac{+}{U A S-s h i^{t s}} \quad / \quad \frac{y, w^{-}}{-} ; \frac{C y O}{+} ; \frac{+}{U A S-s h i^{t s}} \\
& \text { heterozygous control for } U A S-s h i^{t s}
\end{aligned}
$$

heterozygous control for $N P 1227$ :

$$
w^{-}, y ; \frac{N P 1227}{C y O} ;+\quad / \quad \frac{w^{-}, y}{\neg} ; \frac{N P 1227}{C y O} ;+
$$


B. Fly crosses

\section{Type II inhibitory local interneurons (LN2)}

$$
\begin{gathered}
\searrow N P 2426 ;+;+\quad \sigma^{\nearrow} \frac{w^{-}, U A S-s h i^{t s}}{\neg} ;+\left[U A S-s h i^{t s}\right. \\
\Downarrow
\end{gathered}
$$

$$
\frac{N P 2426}{U A S-s h i^{t s}} ; \frac{+}{+} ; \frac{+}{U A S-s h i^{t s}} \quad / \quad \frac{N P 2426}{7} ; \frac{+}{+} ; \frac{+}{U A S-s h i^{t s}}
$$

test group expressing shibire ${ }^{\text {ts }}$ in LN2 neurons

$$
\begin{gathered}
\bigotimes \quad W^{1118} ;+;+\bigotimes \quad \bigcirc^{\nearrow} \quad N P 2426 ;+;+ \\
\Downarrow \\
\frac{N P 2426}{w^{-}} ; \frac{+}{+} ; \frac{+}{+} \quad / \quad \frac{w^{-}}{7} ; \frac{+}{+} ; \frac{+}{+}
\end{gathered}
$$

heterozygous control for NP2426

$$
\begin{aligned}
& \text { ๖ } W^{1118} ;+;+\bigotimes \bigcirc^{\nearrow} \frac{w^{-}, U A S-s h i^{t s}}{\neg} ;+U A S-s h i^{t s} \\
& \Downarrow \\
& \frac{w^{-}, U A S-s h i^{t s}}{w^{-}} ; \frac{+}{+} ; \frac{w^{-}, U A S-s h i^{t s}}{+} \quad / \quad \frac{w^{-}}{7} ; \frac{+}{+} ; \frac{w^{-}, U A S-s h i^{t s}}{+} \\
& \text { heterozygous control for NP2426 }
\end{aligned}
$$


B. Fly crosses

\section{Flies for confocal imaging}

\section{Type I inhibitory local interneurons (LN1)}

$$
\begin{aligned}
& \searrow w^{-}, y ; \frac{N P 1227}{C y O} ;+\bigotimes \quad \bigcirc^{7} \rightarrow U A S-m C D 8: G F P, U A S-s y b: G F P ;+ \\
& \Downarrow \\
& \frac{y, w^{-}}{w^{-}} ; \frac{N P 1227}{U A S-m C D 8: G F P, U A S-s y b: G F P} ;+ \\
& \text { female flies used to visualize the GAL4-expression pattern of } N P 1227
\end{aligned}
$$

Type II inhibitory local interneurons (LN2)

$$
\begin{aligned}
& \text { Ұ } N P 2426 ;+;+\quad \bigotimes \quad \bigcirc^{\nearrow} \rightarrow \text { UAS - mCD8 : GFP } U A S-s y b: G F P ;+ \\
& \Downarrow \\
& \frac{N P 2426}{w^{-}} ; \frac{U A S-m C D 8: G F P, U A S-s y b: G F P}{+} ;+
\end{aligned}
$$




\section{Curriculum vitae}

\begin{tabular}{l|l} 
Name: & Jonas Barth \\
Date of birth: & 08.12 .1982 \\
Place of birth: & Büttelborn, Germany \\
Nationality: & German \\
Residence: & Theaterstr. 3 \\
& 37073 Göttingen \\
& Germany \\
Email: & JonasBarth@googlemail.com
\end{tabular}

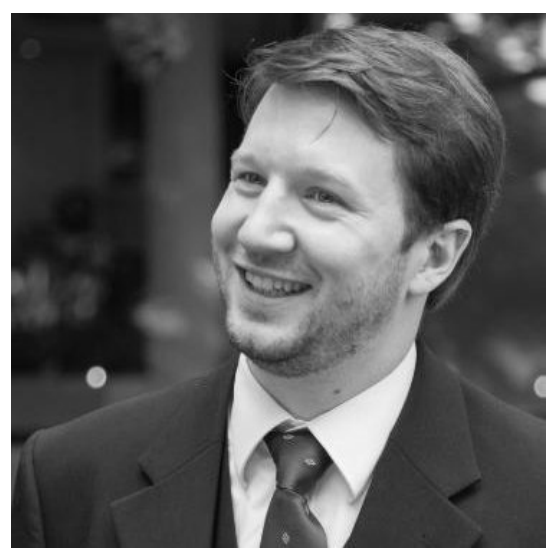

\section{Education}

09/2003 - 09/2007

09/2006 - 02/2007

University of Applied Sciences Giessen-Friedberg graduated as Dipl. Bioinformatician (FH)

Internship in the Department for Cognitive Neuroscience at Radboud University Nijmegen (NL), (Prof. R. Kötter)

Diploma thesis in the Centre for Molecular and Biomolecular

03/2007 - 09/2007 Informatics at Radboud University Nijmegen (NL), (Prof. G. Vriend)

Internship in the Department of Molecular Neurogenetics 03/2008 - 08/2008 at the European Neuroscience Institute Göttingen, (Dr. S. Eimer) 


\section{Curriculum vitae}

\begin{tabular}{|c|c|}
\hline 09/2008 - 09/2009 & $\begin{array}{l}\text { Student of the International Max Planck Research School } \\
\text { Neurosciences }\end{array}$ \\
\hline $01 / 2009-02 / 2009$ & $\begin{array}{l}\text { Lab rotation in the Department of Molecular Biology of Neu- } \\
\text { ronal Signals at the Max Planck Institute for Experimental } \\
\text { Medicine, (Dr. L. Pardo) }\end{array}$ \\
\hline 03/2009 - 04/2009 & $\begin{array}{l}\text { Lab rotation in the Department of Neurogenetics at the } \\
\text { Max Planck Institute for Experimental Medicine, (A. Agarwal, } \\
\text { PhD) }\end{array}$ \\
\hline 05/2009 - 06/2009 & $\begin{array}{l}\text { Lab rotation in the Department of Molecular Neurobiology at } \\
\text { the Max Planck Institute for Experimental Medicine, (Dr. F. } \\
\text { Varoqueaux) }\end{array}$ \\
\hline 09/200 - - 200 & $\begin{array}{l}\text { PhD thesis in the Department of Molecular Neurobiology of } \\
\text { Behavior under supervision of Prof. Dr. André Fiala with the } \\
\text { title: "Olfactory Perception and Physiology in Drosophila } \\
\text { melanogaster" }\end{array}$ \\
\hline
\end{tabular}

\section{Language abilities}

\begin{tabular}{l|l} 
German & Native \\
English & Fluent \\
French & Basic \\
Spanish & Basic
\end{tabular}


C. Curriculum vitae

\section{Conferences and Publications}

\section{Selected conferences}

Barth J, Hermann M, and Fiala A (2011), Olfactory Perception and Physiology in Drosophila melanogaster., $10^{\text {th }}$ Göttingen Meeting of the German Neuroscience Society, Göttingen, Germany, March 23-27, 2011. Poster.

Barth J, Hermann M, and Fiala A (2011), Discrimination and Generalization in Olfactory Learning of Drosophila melanogaster., $12^{\text {th }}$ European Symposium for Insect Taste and Olfaction (ESITO), St. Petersburg, Russia, September 19-25, 2011. Poster.

Barth J, Dipt S, Hermann M, Riemensperger T, and Fiala A (2012), Olfactory discrimination in Drosophila., $8^{\text {th }}$ Federation of European Neuroscience Societies - Forum of Neuroscience, Barcelona, Spain, July 14-18, 2012. Poster.

Barth J, Dipt S, Hermann M, Riemensperger T, and Fiala A (2012), Differential conditioning of similar odors enhances olfactory acuity in Drosophila., $14^{\text {th }}$ European Drosophila Neurobiology Conference, Padua, Italy, September 3-7, 2012. Poster.

Barth J, Dipt S, Hermann M, Riemensperger T, and Fiala A (2013), Enhancement of Olfactory Acuity via Differential Conditioning of Similar Odors., $10^{\text {th }}$ Göttingen Meeting of the German Neuroscience Society, Göttingen, Germany, March 13-19, 2013. Poster.

\section{Publications}

Kucherenko MM, Barth J, Fiala A, and Shcherbata HR (2012), Steroidinduced microRNA let-7 acts as a spatio-temporal code for neuronal cell fate in the developing Drosophila brain., The EMBO Journal, 31(24):4511-4523. 